GEAP-5576

AEC RESEARCH AND DEVELOPMENT REPORT JANUARY 1968

\title{
FINAL SPECIFICATIONS FOR THE SEFOR EXPERIMENTAL PROGRAM
}

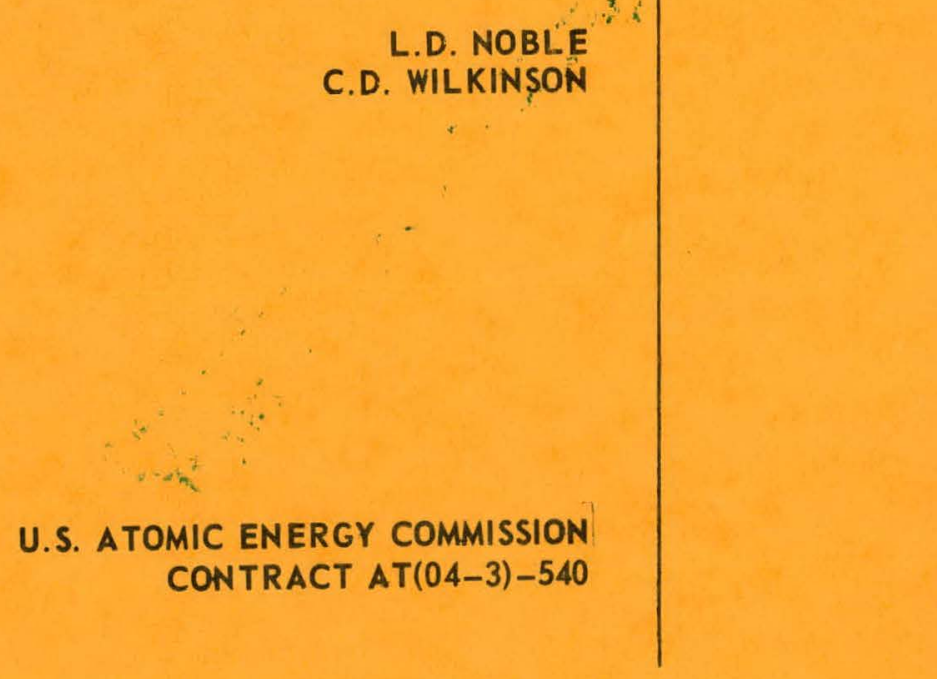

ADVANCED PRODUCTS OPERATION

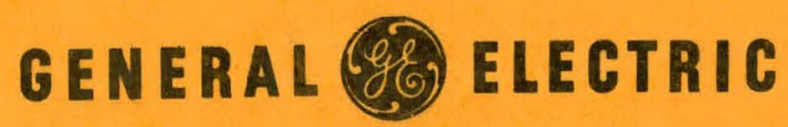




\section{DISCLAIMER}

This report was prepared as an account of work sponsored by an agency of the United States Government. Neither the United States Government nor any agency Thereof, nor any of their employees, makes any warranty, express or implied, or assumes any legal liability or responsibility for the accuracy, completeness, or usefulness of any information, apparatus, product, or process disclosed, or represents that its use would not infringe privately owned rights. Reference herein to any specific commercial product, process, or service by trade name, trademark, manufacturer, or otherwise does not necessarily constitute or imply its endorsement, recommendation, or favoring by the United States Government or any agency thereof. The views and opinions of authors expressed herein do not necessarily state or reflect those of the United States Government or any agency thereof. 


\section{DISCLAIMER}

Portions of this document may be illegible in electronic image products. Images are produced from the best available original document. 


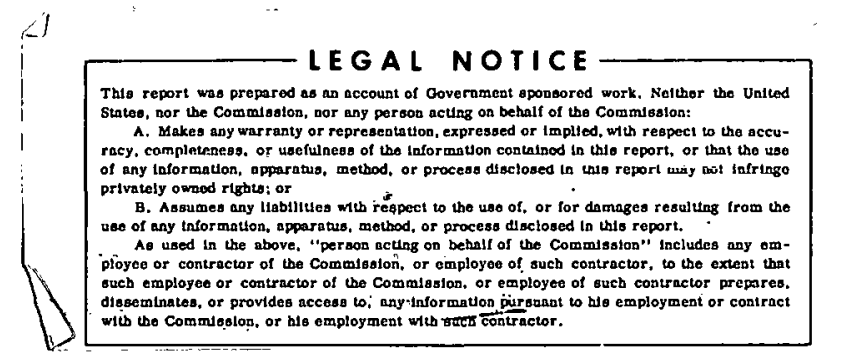

GEAP -5576

AEC Research and Development Report January 1968

FINAL SPECIFICATIONS FOR THE SEFOR EXPERIMENTAL PROGRAM

L. D. Noble

C.D. Wilkinson

APPROVED:

\title{
ER Pflastim
}

G.R. Pflasterer, Development Project Engineer SEFOR Research and Development Program Advanced Products Operation

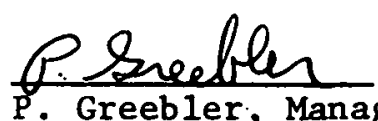

\author{
Prepared for the \\ Southwest Atomic Energy Associates \\ for Transmittal to the \\ U.S. Atomic Energy Commission \\ Under Contract AT (04-3) -540
}

Printed in U.S.A. Available from the

Clearing House for Federal Scientific and Technical Information National Bureau of Standards, U.S. Department of Commerce

Springfield, Virginia

Price: $\$ 3.00$ per copy

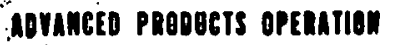 \\ GEÁERAL B? ELETELC \\ SomurnaLe, califanala}

UISTRIBUTION OF THIS DOCUMENT Q URCHERES 


\section{LEGAL NOTICE}

This report was prepared as an account of Government sponsored work. Neither the United States, nor the Commission, nor any person acting on behalf of the Commission:

A. Makes any warranty or representation, expressed or implied, with respect to the accuracy, completeness, or usefuiness of the information contained in this report, or that the use of any information, apparatue, method, or process disclosed in this report may not infringe privately owned rights; or

$B$. Assumes any liabilities with respect to the use of, or for damages resulting from the use of any information, apparatus, method, or process disclosed in this report.

As used in the above, "person acting on behalf of the Cormission" inclucles uny einqloyee or contractor of the Commission, or employee of such contractor, to the extent that such employee or contractor of the Commission, or employee of such contractor prepares, disseminates, or provides access to, any information pursuont to his employment or contract with the Commission, or his employment with ouch contractor. 
GEAP -5576

TABLE OF CONTENTS

Page

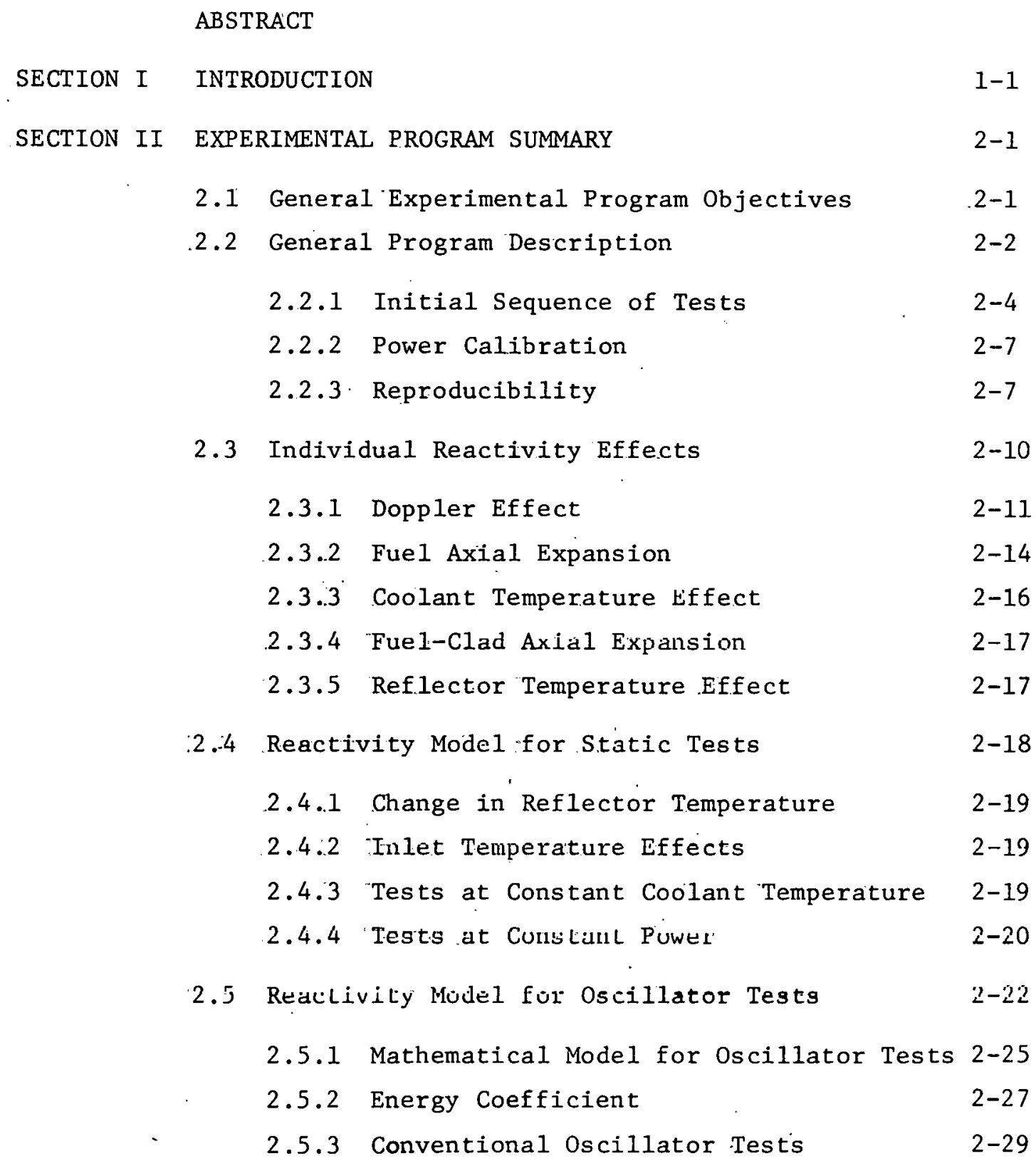


GEAP -5576

TABLE OF CONTENTS (Continued)

$\underline{\text { Page }}$

2.5.4 First Balanced Oscillator Tests 2-31

2.5.5 Second Balanced Oscillator Tests 2-33

2.5.6 Additional Measurements. 2-34

2.6 Reactivity Model for Transient Tests. 2-35

2.6.1 Doppler: Effect.- 2-35

2.6.2 Non-Doppler Feedback 2-36

2.7 Experimental Domain 2-37

SECTION III ZERO POWER TESTS

3.1 Initial Loading to Critical 3-1

3.1.1. Objective 3-1

3.1.2 General Procedure 3-1

3.1.3 Data Collection and Analysis 3-3

3.1 .4 Estimated Results 3-4

3.2 Loading to Full Size Core.. 3-4

3.2.1 Objective 3-4

3.2.2. General Procedure . 3-4

3.2.3 Data Collection and Analysis 3-6

3.2.4 Estimated Résults : 3-6

3.3. Preliminary Calibration of Neutron 3-7

Instrumentation

3.3.1 Objective 3-7

3.3.2 General Procedure 3-7

3.3.3 Data Collection and Analysis 3-11

3.3.4 Estimated Results 3-18 
TABLE OF CONTENTS (Continued)

$\cdots$

Page

3.4 Preliminary Isothermal Temperature

$3-19$ Coefficient

3.4.1 Objective

$3-19$

3.4.2 General Procedure

3-19

3.4.3 Data Collection and Analysis

$3-20$

3.4.4 Estimated Results

3-20

3.5 Source Level Determination.

$3-21$

3.5.1 Objective

$3-21$

3.5.2 General Procedure

3-21

3.5.3 Data Collection and Analysis

3-22

3.5.4 Estimated Results

$3-22$

3.6 Reflector Calibration 3-23

3.6.1 Objective 3-23

3.6.2 General Procedure. 3-23

3.6.3 Data Collection and Analysis 3-25

3.6.4 Estimated Results 3-25

3.7 Material Worth Measurements. 3-25

3.7.1 Objective 3-25

3.7.2 General Procedure 3-27

3.7.3 Data Collection and Analysis 3-28

3.7.4 Estimated Results 3-28

3.8 Flow and Pressure Coefficients of Reactivity 3-29

3.8.1 Objective 3-29

3.8.2 General Procedure 3-29

3.8.3 Data Collection and Analysis 3-30

3.8.4 Estimated Results 3-30 
GEAP-5576.

TABLE OF CONTENTS (Continued)

$\underline{\text { Page }}$

3.9 Loading to Full Power Core $\quad 3-30$

3.9.1 Objective $\quad 3-30$

3.9.2 General Procedure 3-31

3.9.3 Data Collection and Analysis 3-31

3.9.4 Estimated Results 3-32

3.10 Activation Measurements 3-32

3.10.1 Objective 3-32

3.10.2 General Procedure 3-32

3.10.3 Data Collection and Analysis 3-35

3.10.4. Estimated Results 3-38

3.11 Reactor Power Noise Experiment 3-39

3.11.1 Objective 3-39

3.11.2 General Procedure 3-39

3.11.3 Data Collection and Arralysis. 3-41

3.11.4 Estimated Results 3-46

SECTION IV STATIC POWER TESTS 4-1

4.1 Objective 4-1

4.2 General Procedure 4-1

4.3 Data Collection and Analysis 4-3

4.4 Estimated Results 4-7 
GEAP -5576

TABLE OF CONTENTS (Continued)

$\underline{\text { Page }}$

SECTTON V OSCILLATOR TESTS . . 5-1

5.0 'Discussion 5-1

5.0.1. Objectives 5-1

5.0.2 General Procedure 5-2

5.0.3 Data Collection and Analysis 5-5

5.1 Zero Power Oscillator Test 5-13

5.1.1 Objective 5-14

5.1.2 General Procedure 5-14

5.1.3 Data Collection and Analysis 5-15

5.1.4 Estimated Results 5-19

5.2 Conventional Oscillator Tests . 5-24

5.2.1 Ob.jective. 5-24

5.2.2 General Procedure and Test Conditions 5-24

5.2.3 Data Collection, and Analysis 5-27

5.2.3.1 Discussion 5-27

5.2.3.2 Procedure for Fitting of 5-31

5.2.3.3 Effects of Biased Errors . 5-35

5.2.4 Expected Results 5-36

5.3 F.low and Reactivity Oscillations 5-54

5.3.1 Objective $\quad 5-54$

5.3.2 General Procedure and Test Conditions 5-54

5.3.3 Data Collection and Analysis 5-56 
5.4 Balanced Oscillator Tests 5-58

5.4.1 Objective $\quad$ -

5.4.2 General Procedure and Test Conditions 5-59

5.4.3 Data Collection and Analysis 5-62

5.4.3.1 First Balanced Oscillator 5-62 Feedback

5.4.3.2 First Balanced Oscillator - 5-63 Flow Measurements

5.4.3.3 Second Balanced Oscillator 5-64 Tests

5.4.3.4 Fue1 Thermocouple Response 5-65

5.4.4 Expected Results 5-67

SECTION VI TRANSIENT TESTS 6-1

6.0.1 Objective 6-1

6.0.2 Description of Tests 6-1

6.0.3 Data Collection and Analysis 6-2

6.1 Sub-Prompt Critical Transients 6-6

6.1.1 Description of Tests 6-6

6.1 .2 Test Conditions 6-7

6.1.3 Data Collection 'and Analysis 6-9

6.1.4 Expected Results 6-12

6.2 Super-Prompt Critical Transients 6-23

6.2.1 Description of Tests 6-23

6.2 .2 . Test Conditions 6-23

6.2.3 Data Collection and Analysis 6-26

6.2.4 Expected Results 6-28 
TABLE OF CONTENTS (Continued)

$\underline{\text { Page }}$

\begin{tabular}{|c|c|c|}
\hline APPENDIX I & SIMPLIFICATION OF OSCILLATOR REACTIVITY MODEL & $I-1$ \\
\hline \multirow[t]{3}{*}{ APPENDIX II } & ENERGY ABSORPTION IN REACTOR & II -1 \\
\hline & Reactor Energy Balance & II-1 \\
\hline & $\begin{array}{l}\text { Response of Energy Absorption to } \\
\text { a Power Change }\end{array}$ & II -1 \\
\hline \multirow[t]{3}{*}{ APPENDIX III } & OSCILLATOR COOLANT TEMPERATURE RESPONSE & III -1 \\
\hline & III.1 Coolant Temperature in the Core & III -1 \\
\hline & III.2 Coolant Temperature in External Loop & III-11 \\
\hline \multirow[t]{3}{*}{ APPENDIX IV } & $\begin{array}{l}\text { METHODS OF DETERMINING FREQUENCY COMPONENTS } \\
\text { AND EFFECTS OF FINITE DIGITIZING INTERVALS } \\
\text { (ALIASING) }\end{array}$ & IV -1 \\
\hline & Comparison of Two Methods & IV-1 \\
\hline & $\begin{array}{l}\text { Effects of Finite Digitizing Intervals } \\
\text { (Aliasing) }\end{array}$ & IV -7 \\
\hline \multirow[t]{3}{*}{ APPENDIX V } & I.FAST SOৃIIARES CURVE FITTING & $v-1$ \\
\hline & Introduction & $v-1$ \\
\hline & Least Squares Curve Fitting & $V-1$ \\
\hline APPENDIX VI & $\begin{array}{l}\text { ESTIMATED ERRORS IN FEEDBACK FOR OSCILLATOR } \\
\text { TESTS }\end{array}$ & $\mathrm{VI}-1$ \\
\hline
\end{tabular}


TABLE OF CONTENTS (Continued)

$\underline{\text { Page }}$

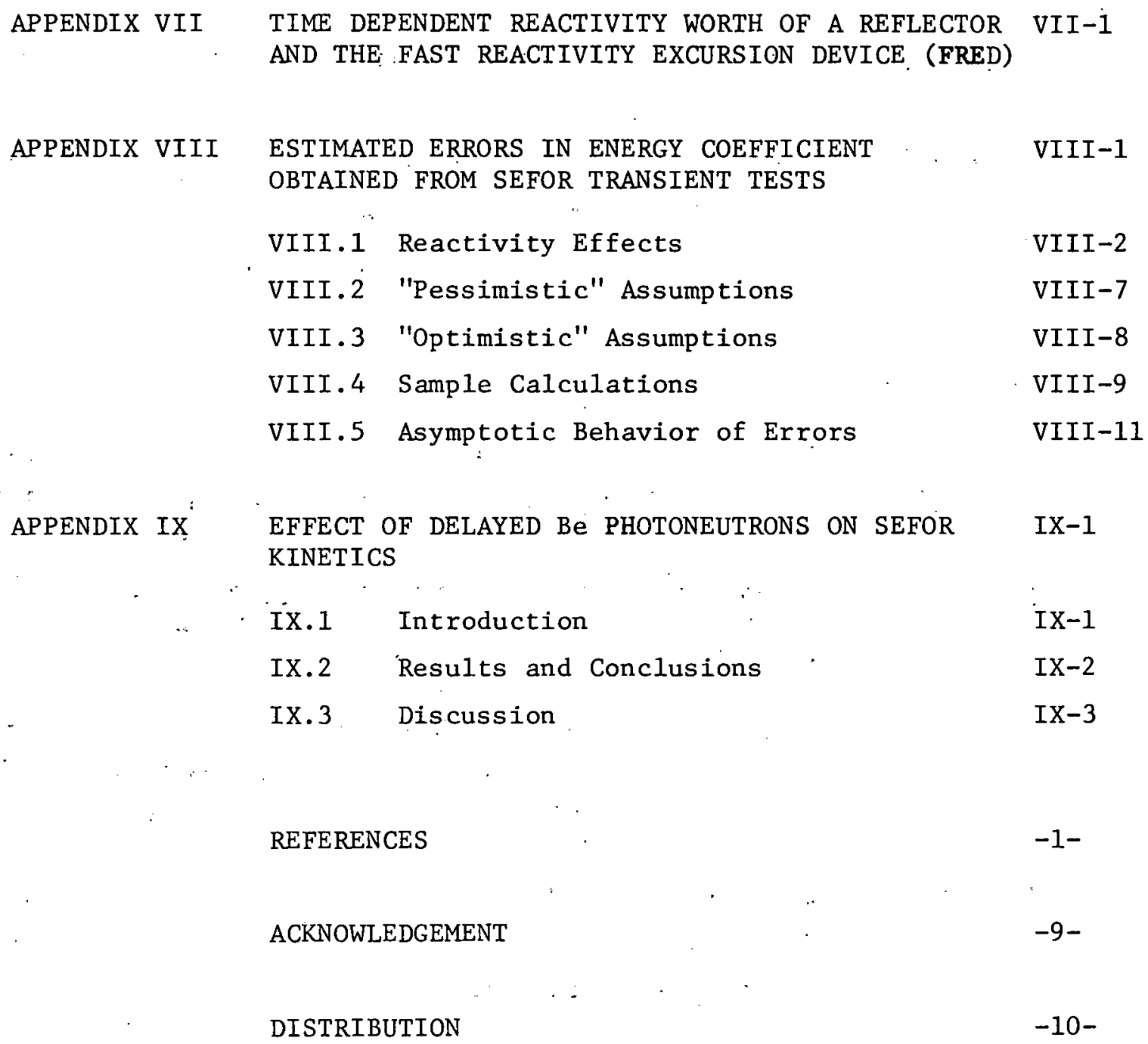


GEAP-5576

\section{LIST OF ILLUSTRATIONS}

Figure

$\underline{\text { Title }}$

$\underline{\text { Page }}$

$2 \cdot 5-1$

Expanded Analytical Model for Oscillator Tests

$2-24$

$2.7-1$

Experimental Domain

2-39

3.1-1

SEFOR Critical Approach, Center Load Method

$3-5$

$3.6-1$

Reflector Worth vs. Position

$3-26$

Reactor Power Noise Measurement

$3-42$

4-1

Static Test Points

$4-8$

4-2

Doppler Reactivity vs. Power

4-9

$5.1-1$

Amplitude for Pure Pu-239, SEFOR, and Pure

$5-20$

U-238 Zero Power Transfer Functions

$5.1-2$

Phase Angle for Pure Pu-239, SEFOR, and Pure U-238 Zero Power Transfer Functions

$5.1-3$

Amplitude of Subcritical Zero Power Transfer Function

$5.1-4$

Phase Angle of Subcritical Zero Power Transfer Functiun

Conventional Oscillator Tests at. Constant Air Flow; Amplitude of Power Reactor Transfer Function at $5 \mathrm{MW}, 30 \%$ Flow

$5.2-2$

Conventional Oscillator Tests at Constant Air Flow; Phase of Power Reactivity Transfer

Function at $5 \mathrm{MW}, 30 \%$ Flow.

$5.2-3$

Conventional Oscillator Tests at Constant Air Flow; Amplitude of Power Reactivity Transfer

Function at $5 \mathrm{MW}, 100 \%$ Flow

Conventional Oscillator Tests at Constant Air Flow; Phase of Power Reactivity Transfer

Function at $5 \mathrm{MW}, 100 \%$ Flow

Conventional Oscillator Teete at Constant Air Flow; Amplitude of Power Reactivity Transfer Function at $10 \mathrm{MW}, 50 \%$ Flow 
GEAP-5576

\section{LIST OF ILLUSTRATIONS (Continued)}

Figure

Title

Page

$5.2-7$

Conventional Oscillator Tests at Constant Air

$5-44$ Flow;Amplitude of Power Reactivity Transfer Function at $10 \mathrm{MW}, 100 \%$ Flow

$5.2-8$

Conventional Oscillator Tests at Constant Air

$5-45$ Flow;Phase of Power Reactivity. Transfer Function at $10 \mathrm{MW}, 100 \% \mathrm{Flow}$

$5.2-9$

Conventional Osciliator Tests at Constant Air Flow;Amplitude of Power Reactivity Transfer Function at $20 \mathrm{MW}, 100 \%$ Flow

$5.2-10$

Conventional Oscillator Tests at Constant Air Flow; Phase of Power Reactivity Transfer Function at $20 \mathrm{MW}, 100 \%$ Flow

5.2-11 Conventional Oscillator Tests at Constant IHX Secondary. Inlet Temperature;Amplitude of Power Reactor Transfer Function at $5 \mathrm{MW}$

Conventional Oscillator Tests at Constant IHX Secondary Inlet Temperature; Phase Angle of Power Reactivity Transfer Function at $5 \mathrm{MW}$

Conventional Oscillator Tests at Constant IHX Secondary Inlet Temperature;Amplitude of Power Reactivity Transfer Function at io MW

$5 \cdot 2-14$

Conventional Oscillator Tests at Constant IHX Secondary Inlet Temperature; Phase Angle of Power Reactivity Transfer Function at $10 \mathrm{MW}$

Conventional Oscillator Tests at Constant IHX Secondary Inlet. Temperature; Amplitude of Power Reactivity Transfer Function at $20 \mathrm{MW}, 100 \%$ F1ow Power Reactivity Transfer Function at $20 \mathrm{MW}$, $100 \%$ F1ow 


\section{LIST OF ILLUSTRATIONS (Continued)}

5.4-1 First Balanced Oscillator Tests

Amplitude of Power Reactivity Transfer Function at $5 \mathrm{MW}$

First Balanced Oscillator Tests

Phase of Power Reactivity Transfer

Functiọn at $5 \mathrm{MW}$

First Balanced Oscillator Tests Amplitude of Power Reactivity Transfer Function at $10 \mathrm{MW}$

First Balanced Oscillator Tests

Phase of Power Reactivity Transfer

Function at $10 \mathrm{MW}$

5.4-5 First Balanced Oscillator Tests

Amplitude of Power Reactivity Transfer Function at $20 \mathrm{MW}$

First Balanced Oscillator Tests Phase of Power Reactivity Transfer Function at $20 \mathrm{MW}$

5.4-7 Second Bàlanced Oscillator Tests Amplitude of Reactivity to Average Coolant Temperature Transfer Function 
GEAP-5576

\section{LIST OF ILLUSTRATIONS (Continued)}

Figure

6. $1-6$

6.1-7

6.1-8

6.1-9

6.2-1

$6.2-2$

$6.2-3$

$6.2-4$

6.2-5

6.2-6

6.2-7

6.2-8

$6.2-9$

III-1

III-2.

III -3

I II -4

III-5

IV -I

IV -2
Title

$\underline{\text { Page }}$

\$0.96 Transient at $15 \mathrm{MW}$ for Calculated Coefficients

$6-19$

\$0.96 Transient at $5 \mathrm{MW}$ for - -.004 Doppler 6-20

$\$ 0.96$ Transient at. $10 \mathrm{MW}$ for -.004. Doppler 6-21

\$0.96 Transient at 15 MW for - -.004 Doppler 6-22

\$1.10 Transient at $0.1 \mathrm{MW}$ for Calculated 6-29 Coefficients

$\$ 1.10$ Transient at $5 \mathrm{MW}$ for Calculated $6-30$

Coefficients

\$1.10 Transient at $10 \mathrm{MW}$ for Calculated 6-31

Coefficients

$\$ 1.30$ Transient at $0.1 \mathrm{MW}$ for Calculated. 6-32

Coefficients

\$1.30 Transient at $5 \mathrm{MW}$ for Calculated 6-33

Coefficients

$\$ 1.30$ Transient at $10 \mathrm{MW}$ for Calculated 6-34

Coefficients

$\$ 1.30$ Transient at $0.1 \mathrm{MW}$ for -.004 Doppler 6-35

\$1.30 Transient at $5 \mathrm{MW}$ for -.004 Doppler 6-36

$\$ 1.30$ Transient at $10 \mathrm{MW}$ for -.004 Doppler 6-37

Amplitude of the Coolant Temperature III-6

Functions $\mathrm{F}_{i}$ and $\mathrm{F}_{0}$ for SEFOR at 100\% Flow

Phase Angle of the Coolant Temperature Functions III-7 $\mathrm{F}_{i}$ and $\mathrm{F}_{0}$. for SEFOR at $100 \%$ Flow

Error in Amplitude of Approximate Expressions III-8

for $F_{i}$ and $F_{0}$ in SEFOR

Error in Phase Angle of Approximate Expressions III-9 for $F_{i}$ and $F_{0}$ in SEFOR

Model for Frequency Response of Average Coolant III-10

Temperature to a Power Oscillation

$f(\omega)$ and $h(\omega)$ when $N=6$

IV -5

$g(\omega)$ and $r(\omega)$ when $N=6$

IV -6 


\section{IIST OF ILLUSTRATIONS (Continued)}

Figure

VI-1

VI-2

VII-1

VII-2

VII-3

VII-4

VII-5

VII-6

VII-7

VIII-1

VIII-2
Title

Estimated Deviation in Real Part of the Feedback (Conventional Oscillator. Tests)

Estimated Deviation in Imaginary Part of the Feedback (Conventional Oscillator Tests)

Estimated Reflector Position vs Time

Reflector Worth vs Position for 2-Segment Core

Estimated Reflector Worth ( $\$ 1.3$ total) as a Function of Time

Slug Worth vs Position - Normalized at $100 \mathrm{c}$ at Flux Peak ( -3.0 inches)

FRED Displacement vs Time

Time Dependent FRED Worth for 20-Inch Travel Time of $95 \mathrm{msec}$

Time Dependent FRED Worth for 20-Inch Trave1 Time of $65 \mathrm{msec}$

Error in Energy Coefficient. vs FRED Worth

Reactor Power With and Without Reflector Motion, Complete FRED Ejection in $90 \mathrm{msec}$
Page

VI-4

VI-5

VII-3

VII-4

VII -5

VII -6

VII-7

VII-8

VII-9

VIII-3

VIII -4 


\section{LIST OF TABLES}

Table

1-1

$1-2$.

2-1

3-1

3-2

4-1

4-2

5-1

5-2

5-3

5-4

5-5

6-1

6-2

6-3

6-4

II-1

II-2

VIII -1

VIII-2

VIII-3 $\underline{\text { Title }}$

Page

Initial Test Sequence

2-6

Effect of Fission Product Decay Heat

2-9

Calculated Coefficients (10 MW)

$2-21$

Calculated Neutron Spectrum in FRED

$3-12$

Channel (Case C4HR1)

Core Average Cross Section Ratios

3-39

Equilibrium Conditions for Static Tests

4-4

Calculated Coefficients; Non-Spatially

Weighted Models

4-10

$5-26$

5-27

Oscillator Tests

Flow and Reactivity Oscillations

5-55

Balanced Oscillator Tests

5-61

Possible Frequencies For Balanced

$5-61$

Oscillator Testo

Sub-Prompt Critical Familiarization Tests

6-8

Sub-Prompt Critical Transients

6-9

Super-Prompt Critical Transients ( $\left.T \frac{\mathrm{dk}}{\mathrm{d} T}-.004\right)$

$6-24$

Super-Prompt Critical Transients ( $\left.\mathrm{T} \frac{\mathrm{dk}}{\mathrm{dT}}-.008\right)$

$6-25$

II-2

Reactor Energy Balance

II -2

Energy Absorption Fractions

VIII-9

FRED out at $t=90 \mathrm{msec}$ )

Case S-158 ( $\$ 1.10$ Slug - Reflector Motion

$50 \mathrm{msec}$ before FRED - FRED out at $t=140 \mathrm{msec}$ )

VIII-9

VIII-10 
GEAP -5576

ABSTRACT

The SEFOR experimental program is comprised of a series of tests beginning with low.power physics tests,.,proceeding through static and frequency response tests at vartous power levels up to full power, and culminating in a series of sub-prompt and superprompt critical transients.

The work reported here describes an initial sequence of tests, detalled speciffcations of the number of tests, and the reactor operating conditions (such as power temperature, flow rates, and reactivity changes) for each test, as well as detailed spectification of data reduction and interpretation procedures and estimated results for each of the tests. 
GEAP -5576

\subsection{INTRODUCTION}

The Southwest Experimental Fast Oxide Reactor (SEFOR) is a $20 \mathrm{MW}(t)$. fast spectrum reactor fueled with mixed $\mathrm{PuO}_{2}-\mathrm{UO}_{2}$ and cooled with sodium. SEFOR will have characteristics similar to large, soft spectrum fast breeder reactors fueled with mixed $\mathrm{PuO}_{2}-\mathrm{UO}_{2}$. Economic studies of these large power reactors indicate significant potential for producing low cost power. SEFOR will be used to obtain physics and engineering data at fuel compositions, temperatures, and crystalline states characteristic of power reactor operating conditions. SEFOR is particularly designed for the systematic determination of the Doppler coefficient of reactivity at temperatures up to the vicinity of fuel melting.

The SEFOR Project consists of two major parts: the design and construction of the reactor and a related research and development program. Funds for the design and construction of the facility are beling pruvided by the Southwest Atomic Energy Associatcs (a group of seventeen investor-owned utility companies located in the South and Southwest part of the United States), together with the Karlsruhe Laboratory of the Federal Republic of Germany, Euratom, and the General Electric Company.

The United States Atomic Energy Commission is supporting the research and development program. The $R$ and $D$ Program consists of two phases:

Phase I - Pre-Operationa.1. Research and Development

Phase II - Post Construction Research and Development. Work performed in Phase I of the program under Task 1.i, Specifications for the Reactor, and Experiment Equipment, has been reported in reference 1 and 2 . 


\section{GEAP -5576}

This report describes work performed in Phase I of the program under Task 1.2., Final Specifications of the Experimental Program, in the period November, 1965 through April, 1967... The. purpose of Task. 1.2

was to conduct detailed analysis necessary for preparing, and to prepare, final specifications of the experimental program with known. reactor and equipment specifications. 
GEAP-5576

' SECTION II

\section{EXPERIMENTAL PROGRAM SUMMARY}

2.1 GENERAL EXPERIMENTAL PROGRAM OBJECTIVES

The objectives of the SEFOR experimental program are to:

a. Investigate the operating characteristics of sodiumcooled, mixed $\mathrm{PuO}_{2}-\mathrm{UO}_{2}$ fueled fast spectrum reactors.

b. Investigate and measure the Doppler reactivity effect during normal reactor operating conditions.

c. Investigate and measure the Doppler reactivity effect during abnormal reactor transient conditions, and in particular, to determine its relation to inherent reactor shutdown capability.

The Doppler reactivity effect is one of several significant reactivity feedback effects present in SEFOR, which in the general case, act simultaneously. Thus, to evaluate the Doppler effect quantitatively, it is necessary that the experimental program objectives include investigation and measurement of all other significant reactivity effects. 
GEAP -5576

\subsection{General Program Description}

The experiments which have been. planned for the first core of the SEFOR reactor are described in detail in the following sections. Similar tests, but fewer in number, will be performed in the second core. Earlier descriptions of the experimental program appear in references 1 to 5 .

The tests have been broken down into the following four broad classifications: 1) zero power tests,2) static tests, 3) oscillator tests (conventional and balanced), and coolant flow oscillations, and 4) transient tests. Except for the balanced oscillator tests and the sinusoidal coolant flow oscillations, all of the proposed experiments are the same, or similar, to tests which have been successfully performed on other reactors. The balanced oscillator tests are not essential to the successful execution of the SEFOR experimental program. However, they may provide additional, independent checks on the analysis of other experiments, and the balanced oscillator tests will be attempted on the SEFOR reactor.

1. The zero power tests are conducted at low (zero) power and include conventional tests such as the approach to critical, control rod calibration, power distribution measurements, and material worth measurements. In addition, it is planned that the isothermal temperature coefficient of reactivity will be measured by using an external (non-nuclear) heat source, and that the ratio of the prompt neutron lifetime to the delayed neutron fraction $(\ell / \beta)$ will be determined by noise analysis methods. 
GEAP -5576

2. In the static tests, the reactor is operated at a steadystate condition at a number of different reactor conditions (i.e. at different power levels, flow rates, and coolant inlet temperatures). The planned test conditions were selected so that the "static" reactivity effects associated with 1 ) a change in power at constant average coolant temperature, 2) a change in average coolant temperature at constant power, and 3) a change in outlet temperature at constant power and average coolant temperature can be separately determined.

3. Two types of oscillator tests are planned. These are: A) Conventional (including zero power) oscillator tests in which a poison rod is oscillated at several frequencies and the reactor power and coolant temperatures are allowed to oscillate about their mean value. B) Balanced oscillator tests (of which there are two types ${ }^{(6)}$ ) in which a poison rod, the primary coolant flow rate, and the.secondary coolant tlow rate are simultaneously oscillated. The relacive phase angle and amplitudes of the reactivity and flow oscillations are. adjusted so that one of the following conditions is satisfied.

a). The inlet and outlet core coolant temperatures remain constant. This is a first balanced oscillator test.

b). The reactor power remains constant. This is called a second balanced oscillator test.

The primary and secondary coolant flow. will be independently oscillated, with no attempt to "balance" the power or coolant temperature. These oscillations will serve to demonstrate reactor stability 
GEAP -5576

to flow' (temperatüre) oscillations and will be used to predict the rélative phase angles and amplittudes of oscillations required for the balanced oscillator tests.

4. The transient tests include sub-prompt critical tests (rapid insertion of " $\$ 1.0$ positive reactivity) and super-prompt critical tests (rapid insertion of > $\$ 1.0$ positive reactivity). These tests will be performed at different initial power levels and will allow determination of the reactivity-energy coefficient over a range of fuel temperatures.

\subsubsection{Initial Sequence of Tests}

A sequence of tests that can be used during the first portion of the planned experimental program is outlined below. "It is expected that the initial tests will be followed by the balanced oscillatior tests and the remainder of the static tests which are described in this report. These would, in turn, be followed by: the sub-prompt critical tests and finally by super-prompt critical tests.

The initial test sequence describe below provides:

A. A safe approach to full power..

Static and oscillator tests are performed at lower power before'proceeding to higher power levels, and checks of previous tests are periodically performed in order to observe any changes: (due to fuel pellet cracking, for example) that'may occur: as a result of operation at high power. 
B. An overall understanding of the reactor at a reasonably early stage in the experimental program. Upon completion of the initial tests, the reactor will have been operated over essentially the complete range of power levels, flow rates, and coolant temperatures.

C. Information on "as fabricated" fuel.

Several tests are performed while maintaining the power level at or below $5 \mathrm{MW}$. The peak fuel temperature is low ( $\left.\leqslant 1200^{\circ} \mathrm{F}\right)$ during these tests and the fuel pellets remain essentially "as fabricated." After operation at high power, these tests may be repeated in order to observe any changes that occur.

D. Information for predicting the relative phase angles and amplitudes required for balanced oscillator tests.

This information is obtained from the flow and reactivity oscillations at 5 and $15 \mathrm{MW}$.

The outline of the initial test sequence is given in Table 1-1. The specified tests and the general procedure for each test are described in other sections of this report. The outline is not intended to serve as a detailed procedure or description of the exact sequence of tests, but rather to serve as a guide for accomplishing points A through $\mathrm{D}$ above. 
GEAP-5576

TABLE 1-1

INITIAL TEST SEQUENCE

$\overline{\mathrm{T}}_{\mathrm{c}}$ (average coolant temperature) $\simeq 760^{\circ} \mathrm{F}$ unless otherwise indicated

POWER (MW.)

0

$1 \rightarrow 2$

5

1

5

10

5

10

15

10

15

19

20

10 or 15
TESTS:

Perform the zero power tests described in Section 3.0;

- Perform the zero power oscillator test $\left(\overline{\mathrm{T}}_{\mathrm{c}} \simeq \sim 400^{\circ} \mathrm{F}\right.$ to $\sim 700^{\circ} \mathrm{F}$ )

Obtain static equilibrium; Obtain a heat balance; Perform a conventional oscillator test

Obtain static equilibrium at 30,50 , and $100 \%$ flow; Obtain a heat balance; Measure the reflector temperature coefficient of reactivity; Perform conventional oscillator tests at 30,50 , and $100 \%$ flow; Perform primary and secondary coolant flow oscillations at $30 \%$ flow; Obtain static equilibrium at 3 coolant:" temperatures between $\sim 500^{\circ} \mathrm{F}$ and $760^{\circ} \mathrm{F}$

Check the previous 1(2) MW static and oscillator test" results

Check a previous 5 MW static equilibrium condition

Obtain static equilibrium at 50 and $100 \%$ flow;

Obtain :a heat balance; Measure the reflector temperature coefficient of reactivity; Perform conventional oscillator tests at 50 and $100 \%$ flow

Check a previous 5. MW static and oscillator test result

Check a previous 10. MW static equilibrium condition

Obtain static equilibrium at 60 and $75 \%$ flow; Obtain. a heat balance; Measure the reflector temperature coefficient of reactivity; Perform a conventional oscillator test at $7.5 \%$ flow; Perform reactivity and: primary and secondary coolant flow oscillations at $60 \%$ flow

Check a previous "10 "M" static and oscillator test résult Check a previous 15 MW static equilibrium condition" Obtain static equilibrium at 95\% flow; Perform a conventional oscillator test at $95 \%$ flow

Obtain static equilibrium; Obtain a heat balance; Measure the reflector temperature coefficient of reactivity.

Check a previous static and oscillator test result 
GEAP-5576

\subsubsection{Power Calibration}

It is expected that the reactor power level in SEFOR will be measured primarily by means of calibrated neutron flux monitors. These monitors will be calibrated by means of a reactor heat balance which relies on measurements of the cool.ant fiow rate and the coolant temperature rise across the reactor. The estimated error in the absolute power calibration at high $(\geq 5 \rightarrow 10 \mathrm{MW})$ power levels is $\sim \pm 5 \%$, (e.g. references 1 and 2 ).

It has been assumed throughout this report that the flux monitors are calibrated in terms of the actual energy, including the equilibrium fission product heating, that is produced in the core. (See Appendix: II for a discussion of the relation between the energy produced and the energy absorbed in various regions of the reactor.) The actual calibration used in the SEFOR experiments is unimportant. However, the relationships described in Appendix II should be kept in mind when the energy distribution in the reactor (e.g. the fraction of the energy that is absorbed in the fue1) is of. interest.

\subsubsection{Reproducibility}

The expected reproducibility of the measurements are as follows:

\section{Fine Reflectors}

Reproducibility $s \pm 0.5 c$ per reflector 


$$
\text { GEAP- } 5576
$$

\section{Coarse Reflectors}

$$
\text { Reproducibility } \leqslant \pm 0.5 € \text { per reflector }
$$

\section{Coolant Temperatures:}

Reproducibility $\leqslant \pm 3^{\circ} \mathrm{F}$ in the core temperature rise at full power $\left(120^{\circ} \mathrm{F}\right.$ core $\left.\Delta \mathrm{T}\right)$. The core outlet temperature measured by the instrumented fuel assemblies may be "noisy" due to mixing in the cross flow area.

Power:

The flux monitors will accurately reproduce the same current for the same:measured flux level. However, in order to account for the different time behavior of the "fission" heating and the fission product "decay" heating, a record should be kept.. of the operating ${ }^{\circ}$ ime at each power level and the time required to change from one power level to another. These times ean then be used. with: standard fission product decay heat curves : to calculate the actual heat produced in the core. Examples of differences between indicated power levels (i.e. power levels inferred from neutron monitors which are calibrated as described in Section 2.2.2) and the actual power being produced in the core are shown in Table 1.2. In calculating the values shown in this table, it was assumed that the reactor was rapidly brought from a low power to the "initial". power level; that the indicated power level was held constant for two hours and that the reactor power was then rapidiy changed to the new power, and the indicated power level was held constant for 10 minutes. 
GEAP -5576

TABLE $1-2$

\section{EFFECT OF FISSION PRODUCT DECAY HEAT}

\begin{tabular}{|c|c|c|c|}
\hline $\begin{array}{l}\text { Indicated Initial } \\
\text { Power Level After } \\
2 \text { Hours Operation }\end{array}$ & $\begin{array}{l}\text { Actual Initial } \\
\text { Power Produced } \\
\text { In Core After } \\
2 \text { Hours Operation }\end{array}$ & $\begin{array}{l}\text { Indicated New } \\
\text { Power Leve1 After .. } \\
10 \text { Minutes Operation }\end{array}$ & $\begin{array}{l}\text { Actual New } \\
\text { Power Produced } \\
\text { in Core After } \\
\text { 10. Minutes Operation }\end{array}$ \\
\hline (MW) & $(\mathrm{MW})$ & $(\mathrm{MW})$ & (MW) \\
\hline 6.00 & 5.92 & 4.00 & 3.97 \\
\hline 4.00 & 3.95 & 6.00 & 5.90 \\
\hline 1.00 & 0.99 & 10.00 & 9.77 \\
\hline 10.00 & 9.87 & 1.00 & 1.09 \\
\hline
\end{tabular}

Fuel Thermocouples

The thermocouple junction should accurately produce the same current for the same junction temperature. However, the relationship between the thermocouple junction temperature (fuel temperature) and power may change when the fuel temperature is raised above the sintering temperature $\left(\sim 3000^{\circ} \mathrm{F}\right)$. The change in this relationship above the sintering temperature depends on the fuel t1me-temperature history. It is expected that an equilibrium fuiel structure in a fuel pellet can be generated within $\sim 40$ hours at fuel temperatures above $\sim 4000^{\circ} \mathrm{F}$. The relationship between the thermocouple junction temperature and the measured power level in such a pellet should remain unchanged thereafter. Tests (e.g. reference 7 - Section 6.1.3.2) 
GEAP-5576

indicate that after sintering occurs, the gunction temperature for the same power level may decrease by $10 \%$ for temperatures in the range $4000 \rightarrow 4500^{\circ} \mathrm{F}$, and that the junction temperature may increase by $15 \%$ for temperatures in the $1500^{\circ} \mathrm{F}$ range.

\section{Flow Meters}

The expected reproducibility of the flow meters in the instrumented fuel assemblies is $\pm 5 \%$ for flow rates above $25 \mathrm{gpm}$. (full flow $=60 \mathrm{gpm}$ ) and $\pm 10 \%$ for flow rates in the $10 \rightarrow 25 \mathrm{gpm}$ range. The expected reproducibility of the Venturi flow meter and the large electromagnetic flow meter in the primary coolant inlet line is of the order of $\pm 1 \%$.

The actual reproducibility of the instrumentation and equipment in SEFOR should be checked by performing the same tests a number of times.

\section{$2.3 \quad$ Individual Reactivity Effects}

Analyses indicate that feedback reactivity changes in SEFOR w111. be primarily dependent on the Doppler (fuel temperature), core average coolant temperature, and the core structure temperature. Other feedback reactivity effects, believed to be of secondary importance include fuel axial expansion (fuel temperature), fuel-clad axial expansion, and reflector temperature effects. 
GEAP -5576

Determination of the Doppler effect, and hence, the Doppler reactivity coefficient, is a primary objective of the SEFOR experimental program. Evaluation of a single feedback effect is complicated by the fact that most reactor experiments involve several feedback effects simultaneously. Thus, the SEFOR experimental program is designed to evaluate the composite reactivity feedback over the range of reactor conditions of interest in such a manner that the individual effects, and hence, the Doppler effect, may be separated or "backed out" of the experimental data. Separation of the various reactivity. effects cannot be accomplished exactly because of practical experimental limitations and imperfect prediction of theoretical models for the various effects. Fortunately, in the case of SEFOR,..different types of experiments (e.g. static, oscillator, and transient) can be used to obtain independent evaluations of some of the more important feedback effects.

Recommended models for each of the reactivity feedback effects are described in the following sections. It is expected that these feedback models will adequately describe SEFOR behavior so as to afford a good fit to the experimental data.

\subsubsection{Doppler Effect}

Calculations (7) have shown that for SEFOR the Doppler effect, $\Delta \mathrm{k}_{\mathrm{D}}$, may he closely approximated by: .

$$
\Delta k_{D}=\frac{\int p^{2}(r) f(r) d V}{\int P^{2}(r) d V}
$$

where: $P(r)$ is the local power density, and where the range of Integralion extends over the fucl volume. 
$f(r)=$ function of the change in local fuel temperature, $T_{f}(r)$.

A local Doppler coefficient, $\Gamma$, may be defined such that:

$$
f(r):=\underset{\Delta T(r)}{\int} \mathrm{dT}_{f}=\int_{\Delta T(r)}\left[\frac{\mathrm{dk}}{\mathrm{dT}}\right]_{f} \mathrm{dT}_{\mathrm{f}}:=\Delta \mathrm{k}_{\mathrm{D}}(\mathrm{r})
$$

The local Doppler coefficient may be represented as:

$$
\Gamma=\frac{A}{T_{f}^{m}}
$$

Substitution and integration over a change in fuel temperature from $T_{f 1}(r)$ to $T_{f 2}(r)$ gives:

$$
\begin{aligned}
\Delta k_{D} & =\frac{A}{1-m} \frac{\int V_{\text {Fuel }} P^{2}(r) \Delta\left[T_{f}(r)^{1-m_{1}}\right] d V}{\int V_{\text {Fuel }} P^{2}(r) d V}(m \neq 1) \\
& \equiv A F_{m}\left(\Delta T_{c}, \Delta P\right)
\end{aligned}
$$

where: $F_{m}\left(\Delta T_{i c}, \Delta P\right)$, [which can be calculated by , the WEDOP code ${ }^{(12)}$ ], depends on the change in local fuel temperature (and thus on the change in average coolant temperature and power level) as we11 as the functional form (the value of $: m$ ) that is assumed for the local Doppler effect. If $\mathrm{m}=1,(2.3-4 \mathrm{a})$ reduces to:

$$
\begin{aligned}
\Delta \mathrm{k}_{\mathrm{D}} & =\frac{\mathrm{A} \int \mathrm{V}_{\text {Fuel }} \mathrm{P}^{2}(\mathrm{r}) \ln \frac{\mathrm{T}_{\mathrm{f} 2}(\mathrm{r})}{\mathrm{T}_{\mathrm{f} 1}} \mathrm{dV}}{\int \mathrm{V}_{\text {Fuel }} \mathrm{P}^{2}(\mathrm{r}) \mathrm{dV}} \quad(\mathrm{m}=1) \\
& =A F_{1}\left(\Delta \mathrm{T}_{\mathrm{c}}, \Delta \mathrm{P}\right)
\end{aligned}
$$


It is also planned to represent the Doppler effect by a less complicated form that is more useful for reactor operating purposes. Calculations indicate that one of the following forms may be useful in this regard:

$$
\begin{aligned}
& \Delta \mathrm{k}_{\mathrm{D}}=\frac{\mathrm{A}}{1-\mathrm{m}} \Delta\left[\mathrm{T}_{\mathrm{f}}^{\left.1-\mathrm{m}_{1}\right]}, \quad \ldots \quad(\mathrm{m} \neq 1)\right. \\
& \Delta \mathrm{k}_{\mathrm{D}}=\mathrm{A} \ln \frac{\mathrm{T}_{\mathrm{f} 2}}{\mathrm{~T}_{\mathrm{f} 1}}=\mathrm{A} \ln \left[1+\frac{\Delta \mathrm{T}_{\mathrm{f}}}{\mathrm{T}_{\mathrm{f} 1}}\right] \quad(\mathrm{m}=1)
\end{aligned}
$$

where: $\quad T_{f}=$ effective fuel temperature $=T_{c}+R_{f} P$

$$
: \Delta T_{f}=\text { change in effective fuel'temperature }=\Delta T_{c}+R_{f} \Delta P
$$

and where:

$$
\begin{aligned}
& T_{c}=\text { average coolant temperature } \\
& R_{f}=\underset{\text { power }}{\text { change in effective fuel temperature with respect to }}
\end{aligned}
$$

For sma11. changes in power and average coolant temperature abour a reference condition ( $\left.\mathrm{T}, \mathrm{co}, \mathrm{P}_{\mathrm{O}}\right)$,

$$
\Delta \mathrm{k}_{\mathrm{D}} \simeq \mathrm{C}_{\mathrm{D}} \Delta \mathrm{T}_{\mathrm{f}}=\mathrm{C}_{\mathrm{D}} \Delta \mathrm{T}_{\mathrm{C}}+\alpha_{\mathrm{D}} \Delta \mathrm{P}
$$

where (for the simple model)

$$
\begin{aligned}
& \left.C_{D} \equiv \frac{A}{\left(T_{C o}+R_{f} P_{o}\right.}\right)^{m} \\
& \left.\alpha_{D} \equiv \frac{A R_{f}}{\left(T_{C o}+R_{f} P_{o}\right.}\right)^{m}
\end{aligned}
$$


or (for the more detailed mode1)

$$
\begin{aligned}
& \left.C_{D} \equiv A \cdot \frac{\partial F_{m}}{\partial T_{c}}\right|_{T_{C O}, P_{o}} \\
& \left.\alpha_{D} \equiv A \frac{\partial F_{m}}{\partial P}\right|_{T_{C o}, P_{0}} .
\end{aligned}
$$

\subsubsection{Fuel Axial Expansion}

The fuel in SEFOR- is made of "dished" pellets and the fuel axial expansion, therefore, depends upon the temperature of the fuel at the shoulder of the dish. The "shoulder temperature" can be expressed as a linear combination of the fuel surface temperature and the average fuel temperature. Thus, the reactivity effect $\Delta K_{e}$. due to the fuel axial expansion can be written as:

$$
\Delta \mathrm{K}_{\mathrm{e}}=\mathrm{C}_{\mathrm{e}} \Delta \mathrm{T}_{\mathrm{e}}=\mathrm{C}_{\mathrm{e}}\left[\mathrm{a} \Delta \mathrm{T}_{\mathrm{fs}}+(1-\mathrm{a}) \Delta \overline{\mathrm{T}}_{\mathrm{f}}\right]
$$

where:

$$
\begin{aligned}
\mathrm{C}_{\mathrm{e}}= & \text { fuel axial expansion reactivity coefficient }\left(\mathrm{c} /{ }^{\circ} \mathrm{F}\right) \\
\Delta \mathrm{T}_{\mathrm{e}}= & \text { change in fuel temperature at the inner edge of the } \\
& \text { shoulder }\left({ }^{\circ} \mathrm{F}\right) \\
\mathrm{a}= & 0.65 \text { for an } 0.875 \text { inch diameter pellet with a } 40 \mathrm{mil} \\
& \text { shoulder } \\
\Delta \mathrm{T}_{\mathrm{fs}}= & \text { change in fuel surface temperature }\left({ }^{\circ} \mathrm{F}\right) \\
\Delta \overline{\mathrm{T}}_{\mathrm{f}}= & \text { change in average fuel temperature }\left({ }^{\circ} \mathrm{F}\right)
\end{aligned}
$$


For a static change, the changes in fuel surface temperature and in average fuel temperature can be expressed in terms of changes in power and average coolant temperature by:

$$
\Delta \mathrm{T}_{\mathrm{fs}}=\mathrm{K}_{\mathrm{s}} \Delta \mathrm{P}+\Delta \mathrm{T}_{\mathrm{c}} \text { and } \Delta \overline{\mathrm{T}}_{\mathrm{f}}=\mathrm{K}_{\mathrm{av}} \Delta \mathrm{P}+\Delta \mathrm{T}_{\mathrm{c}}
$$

where:

$$
\begin{aligned}
\Delta \mathrm{P}= & \text { power change }(\mathrm{MW}) \\
\Delta \mathrm{T}_{\mathrm{C}}= & \text { average coolant temperature change }\left({ }^{\circ} \mathrm{F}\right) \\
\mathrm{K}_{\mathrm{S}}= & \text { change in fuel surface temperature for a small } \\
& \text { power change at a constant average coolant temperature. } \\
\mathrm{K}_{\mathrm{av}}= & \text { change in average fuel temperature for a small } \\
& \text { power change at a constant average coolant temperature. }
\end{aligned}
$$

The quantities $\mathrm{K}_{S}$ and $\mathrm{K}_{\mathrm{av}}$ are related to the "effective" heat transfer coefficient, $h_{f}$ (based on fuel surface area) between fuel surface and coolant, the "average" fuel conductivity $k$, the fuel pellet radius $R$, the fuel length $L$, and the number of fuel rods $N$, by the following expressions.

$$
\begin{aligned}
& \mathrm{K}_{\mathrm{s}}=\frac{\mathrm{A}_{\mathrm{f}}}{2 \pi \mathrm{h}_{\mathrm{f}} \mathrm{RL}}=\frac{\mathrm{A}_{\mathrm{f}} \mathrm{R}}{2 \mathrm{~h}_{\mathrm{f}} \mathrm{V}_{\mathrm{f}}} \\
& \mathrm{K}_{\mathrm{av}}=\mathrm{K}_{\mathrm{s}}+\frac{\mathrm{A}_{\mathrm{f}}}{8 \pi N L \mathrm{k}}=\mathrm{K}_{\mathrm{s}}+\mathrm{A}_{\mathrm{f}} \frac{\mathrm{R}^{2}}{8 \mathrm{k} \mathrm{V}_{\mathrm{f}}}
\end{aligned}
$$

where:

$$
\begin{aligned}
& \mathrm{V}_{\mathrm{f}}=\text { fuel volume } \\
& \mathrm{A}_{\mathrm{f}}=\text { fraction of power which is absorbed in the fuel }
\end{aligned}
$$


GEAP -5576

The reactivity effect due to fuel axial expansion can thus be expressed as:

$$
\Delta K_{e}=C_{e}\left[a_{s}+(1-a) K_{a v}\right] \Delta P+C_{e} \Delta T_{c}
$$

\subsubsection{Coolant Temperature Effect}

It is planned to represent the average coolant temperature effect by:

$$
\Delta \mathrm{k}_{\mathrm{c}}=\mathrm{C}_{\mathrm{c}} \Delta \mathrm{T}_{\mathrm{c}}
$$

where the coefficient $C_{c}$ includes the reactivity effects of sodium density changes in the core as well as temperature coefficients associated with the expansion of core structure; i.e.structure expansion effects should depend primarily on the average coolant temperature.

Calculations ${ }^{(8)}$ indicate that there will be a small (compared to average coolant temperature effect) reactivity effect assoclated with sodium density changes in the sodium between the core and the vessel. This effect should depend primarily on the inlet sodium temperature, $\mathrm{T}_{i}$, and may be represented by:

$$
\Delta \mathrm{K}_{i}=\mathrm{C}_{\mathbf{i}} \Delta \mathrm{T}_{\mathrm{i}}
$$




\subsubsection{Fue1-Clad Axial Expansion}

The temperature of the clad depends on the fuel:surface temperature, $\mathrm{T}_{\mathrm{fs}}$, as well as the coolant temperature. The reactivity effect of the fuel-clad axial expansion may thus be represented by:

$$
\Delta \mathrm{k}_{t}=\mathrm{C}_{\mathrm{t}} \Delta \mathrm{T}_{\mathrm{t}}=\mathrm{C}_{\mathrm{t}}\left[\mathrm{b} \Delta \mathrm{T}_{\mathrm{fs}}+(1-\mathrm{b}) \Delta \mathrm{T}_{\mathrm{c}}\right]
$$

where:

b $<1$ depends on the fuel gap coefficient.

From equation (2.3-11), this can be written as:

$$
\Delta K_{t}-b C_{t} K_{s} \Delta P+C_{t} \Delta T_{c}
$$

for'a static change in power and average coolant.

\subsubsection{Reflector Temperature Effect}

The change of reactivity, $\Delta \mathrm{k}_{r}$, for a change in the average temperature of the reflector, should be of the form:

$$
\Delta \mathrm{k}_{\mathrm{r}}=\mathrm{C}_{\mathrm{r}} \Delta \mathrm{T}_{\mathrm{r}}
$$

where $C_{r}$ is the reflector temperature coefficient. 
GEAP -5576

\subsection{REACTIVITY MODDEL FOR STATIC TESTS}

Using the expressions for the individual reactivity effects that are given in Section 2.3, the composite reactivity feedback may be written (using the simple logarithmic form of the Doppler feedback) as :

$$
K_{f}=K_{o}+A \ln \left[T_{c}+R_{f} P\right]+C_{P} P+C_{q} T_{c}+C_{i} T_{i}+C_{r} T_{r} \quad(2.4-1)
$$

where:

$$
K_{0} \equiv-A \ln \left[T_{c o}+R_{f} P_{o}\right]-C_{P} P_{o}-C_{q} T_{c o}-C_{i} T_{i o}-C_{r} T_{r o} \quad(2.4-2)
$$

where the subscript (o) denotes a reference power or temperature, and where (in terms of the parameters of Section 2.3)

$$
\begin{gathered}
c_{p}: C_{e} C_{s}\left[a K_{s}+(1-a) K_{a v}\right]+b C_{t} K_{s} \\
c_{q}=C_{c c}+C_{t}+C_{e}
\end{gathered}
$$

A change in reactivity between a measured condition and the reference condition can be written as:

$$
\Delta \mathrm{K}_{\mathrm{f}}:=\mathrm{A} \ln \left[1+\frac{\Delta \mathrm{T}_{\mathrm{c}}+\mathrm{R}_{\mathrm{f}} \Delta \mathrm{P}}{\mathrm{T}_{\mathrm{Co}}+\mathrm{R}_{\mathrm{f}} \mathrm{P} \cdot \mathrm{P}}\right]+\mathrm{C}_{\mathrm{P}} \Delta \mathrm{P}+\mathrm{C}_{\mathrm{q}} \Delta \mathrm{T}_{\mathrm{c}}+\mathrm{C}_{\mathrm{i}} \Delta \mathrm{T}_{\mathrm{i}}+\mathrm{C}_{\mathrm{r}} \Delta \mathrm{T}_{\mathrm{f}} \quad(2.4-5)
$$


GEAP-5576

\subsubsection{Change in Reflector Temperature}

If the reflector temperature is changed while the power and coolant temperature are held constant, the change in reactivity between two steady-state conditions is given by:

$$
\Delta \mathrm{K}_{\mathrm{f}}=\mathrm{C}_{\mathrm{r}} \Delta \mathrm{T}_{\mathrm{r}}
$$

\subsubsection{Inlet Temperature Effects}

The inlet temperature reactivity coefficient may be measured by holding the power and average coolant temperature constant. In this case:

$$
\Delta \mathrm{K}=\Delta \mathrm{K}_{\mathrm{f}}-\mathrm{C}_{\mathrm{r}} \Delta \mathrm{T}_{\mathrm{r}}=\mathrm{C}_{\mathrm{i}} \Delta \mathrm{T}_{\mathrm{i}}
$$

where $\Delta \mathrm{K}$ is the measured reactivity change, corrected for any reactivity effect due to changes in the reflector temperature.

\subsubsection{Tests at Constant Coolant Temperature}

- When the average coolant temperature is held constant, equation $(2.4-5)$ reduces to:

$$
\Delta \mathrm{K}=\Delta \mathrm{K}-\mathrm{C}_{i} \Delta \mathrm{T}_{i}-\mathrm{C}_{r} \Delta \mathrm{T}_{r}=\mathrm{A} \ln \left[1+\frac{\mathrm{R}_{\mathrm{f}} \Delta \mathrm{P}}{\mathrm{T}_{\mathrm{co}}+\mathrm{R}_{\mathrm{f}} \mathrm{P}_{\mathrm{o}}}\right]+\mathrm{C}_{\mathrm{p}} \Delta \mathrm{P}
$$

where $\Delta \mathrm{K}$ is the measured reactivity change, corrected for any reactivity effects due to change in inlet temperature or reflector temperature. 
If the more detailed model for the Doppler effect is used (see Section 2.3.1):

$$
\Delta \mathrm{K}=\dot{\mathrm{A}} \mathrm{F}_{\mathrm{m}}\left(\mathrm{T}_{\mathrm{co}}, \Delta \mathrm{P}\right)+\mathrm{C}_{\mathrm{p}} \Delta \mathrm{P}
$$

For small changes in power at a constant coolant temperature:

$$
\Delta K \simeq\left(\alpha_{D}+C_{p}\right) \Delta P \equiv \alpha_{f} \Delta P
$$

where $a_{f}$ is the power coefficient (defined by this equation) and where $\alpha_{D}$ (the Doppler power coefficient) is defined in Section 2.3.

\subsubsection{Tests at Constant Power}

When the power is held constant, the feedback can be written in the form:

$$
\Delta K=A \ln \left[1+\frac{\Delta T_{\dot{c}}}{T_{c o}+R_{f} P_{0}}\right]+c_{q} \Delta T_{c}
$$

of if the more detailed Doppler model is used, as:

$$
\Delta \mathrm{K}=\mathrm{A} \mathrm{F}_{\mathrm{m}}\left(\Delta \mathrm{T}_{\mathrm{c}}, \mathrm{P}_{\mathrm{o}}^{\prime}\right)+\mathrm{C}_{\mathrm{q}} \Delta \mathrm{T}_{\mathrm{c}}
$$

where $\Delta \mathrm{K}$ is defined as before.

For small changes in coolant temperature:

$$
\Delta \mathrm{K} \simeq\left(\mathrm{C}_{\mathrm{D}}+\mathrm{C}_{\mathrm{q}}\right) \Delta \mathrm{T}_{\mathrm{C}}
$$

where $C_{D}$ (the Doppler temperature coefficient) is defined in Section 2.3. 
The calculated ${ }^{(8)}$ reactivity coefficients for the static and oscillator reactivity models at $10 \mathrm{MW}$ and $760^{\circ} \mathrm{F}$ coolant temperature are shown in Table 2-1. As indicated, it is expected that the dominant effects will be due to the power coefficient, the average coolant temperature coefficients, and the fuel temperature coefficient.

TABLE 2-1

CALCULATED COEFFICIENTS (10 MW)

Reactivity Effect or Reactivity Coefficient

Doppler

$A \equiv-0.008 j \Delta \mathrm{k} / \mathrm{k}--2.66 \$$

Power dependence of fuel and fuel clad expansion

Fue1 Temperature Coefficient

Composile Púwer Coefficicnt

Composite Average Coolant Temperature Coefficient

Inlet Temperature.

Reflector Temperature
Temperature Coefficient $\left(c /{ }^{\circ} \mathrm{F}\right)$

$C_{D}=-0.145$

$$
\mathrm{C}_{\mathrm{f}}=-0.149
$$

$$
C_{4}=-0.450
$$$$
c_{i}=-0.029
$$

$C_{r}=-0.04$ $\because$

Power Coefficient (c/Mw)

$$
\alpha_{D}=-8.12
$$

$C_{p}=-0.48$

$a_{\Gamma}=-8.60$ 


\subsection{REACTIVITY MODEL FOR OSCILLATOR TESTS}

In the derivation of the model for the oscillator tests, it is assumed that the amplitudes of the power, reactivity, temperature, and flow oscillations are.sufficiently small, compared to their mean values, that second order terms" can be neglected. With this assumption, the mathematical model for the reactor can be reduced to a system of linear equations with constant coefficients. The model for the Fourier transform of these equations is shown below. Results of conventional oscillator tests are analyzed to determine the overall power-reactivity frequency response. The block diagram below shows the analytical model.

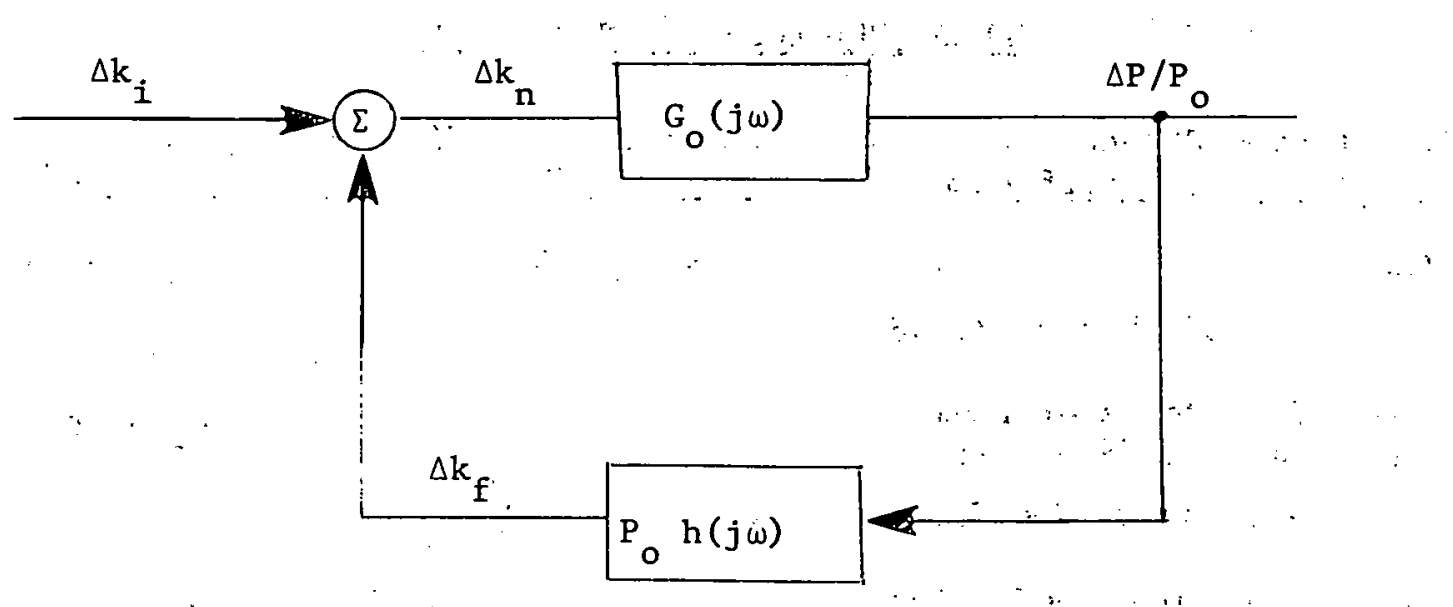

Simple Analytical Model for Oscillator Tësts

The nomenclature used in the block' diagram is defined below.

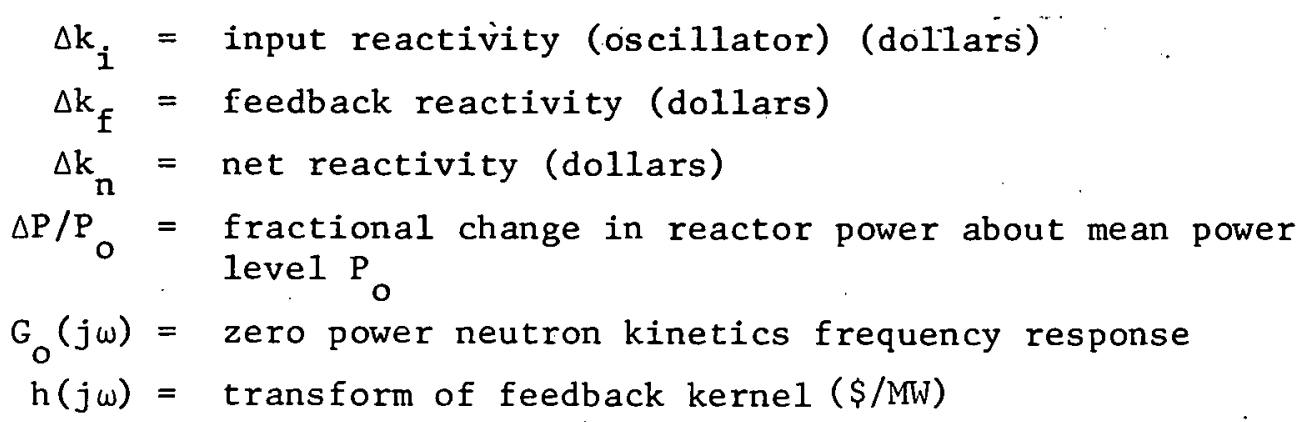


GEAP -5576

Measured quantities are:

$$
\begin{aligned}
G_{0}(j \omega) & =\text { measured at zero power }(0.01 \mathrm{MW}) \\
\frac{\Delta P}{P_{0} \Delta K_{i}} \equiv G_{1}(j \omega) & =\frac{G_{0}}{1-P_{0} G_{0} h}
\end{aligned}
$$

Figure 2.5-1 shows a block diagram of a typical analytical model for SEFOR. (1,2) For clarity, the model is shown for a "point reactor." Actually, the reactivity effects are distributed over the whole reactor. Possible direct effects of inlet and outlet coolant temperature on $\Delta k$ have been omitted. The fuel coefficient $\left(C_{f}\right)$ includes both Doppler and fuel axial expansion effects.

The nomenclature used in Figure 2.5-1 is defined as follows:

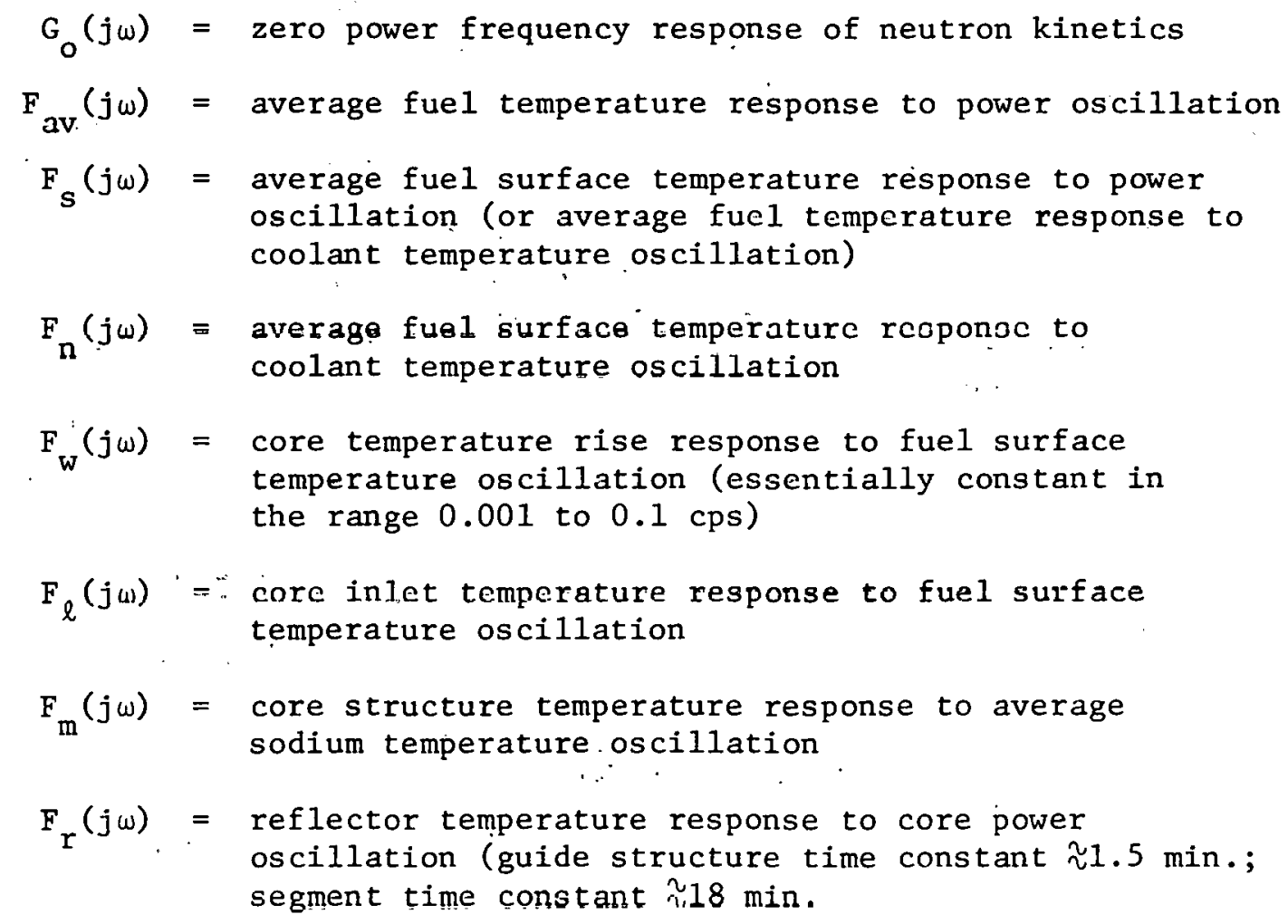




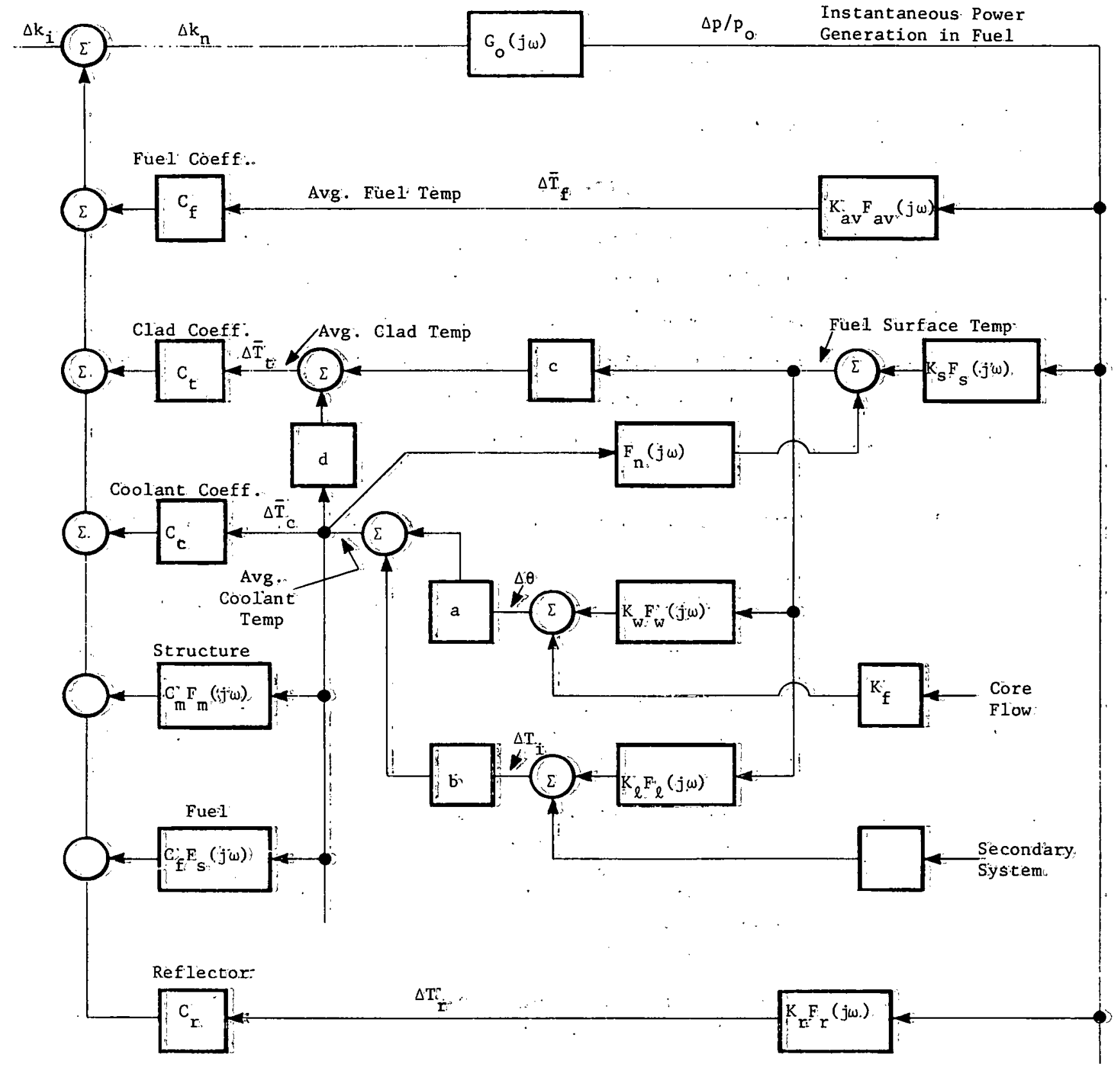

Eigure 2:5-1..- EXPANDED ANADYTICAL. MODEL-FOR? OSCILLATOR TESTS 
The $F(j \omega)$ functions described above are normalized so that the zero frequency values are all 1.0. The " $K$ " values assoclated with each $F(j \omega)$ will vary with power levels, coolant flow rates, and the method of plant control.

\subsubsection{Mathematical Mode1 for Oscillator Tests}

As shown in Appendix I, the reactivity model for the oscillator tests can be approximated by:

$$
\Delta K_{f}(i \omega)=\alpha_{f} F_{a v}(i \omega) x_{f}(i \omega) \Delta P(i \omega)+\left[C_{f} F_{s}(i \omega)+C_{q}\right] \Delta T_{c}(i \omega)+C_{i} \Delta T_{i}(i \omega)
$$

where:

$$
\begin{aligned}
\mathrm{x}_{\mathrm{f}}(\mathrm{I \omega})= & \begin{array}{l}
\text { frequency response of the ratio of the total power } \\
\text { absorbed in the fuel to the "fission" power (normalized } \\
\text { to unity at zero frequency) }
\end{array} \\
\Delta \mathrm{P}(\mathrm{i \omega})= & \begin{array}{l}
\text { "fission" power oscillation (proportional to neutron } \\
\text { fiux) }
\end{array} \\
\Delta \mathrm{K}_{\mathrm{f}}(\mathrm{i} \omega)= & \begin{array}{l}
\text { transform of the total feedback oscillation (corredted } \\
\text { for any effects due to reflector temperature oscillation) }
\end{array}
\end{aligned}
$$

The function $X_{f}(i \omega)$ has been calculated by storrer $(9)$, and is described in more detail in Appendix II.

The average coolant temperature oscillation, $\Delta T_{c}(i \omega)$, is defined in terms of the inlet temperature oscillation, $\Delta T_{i}(i \omega)$, and the outlet temperature oscillation, $\Delta \mathrm{T}_{0}(i \omega)$, by the following:

$$
\Delta \mathrm{T}_{c}(i \omega) \equiv F_{1}(i \omega) \Delta \mathrm{T}_{1}(i \omega)+\left[1-F_{1}(i \omega)\right] \Delta \mathrm{T}_{0}(1 \omega)
$$

where $F_{1} \sim \frac{1}{2}$ is a frequency dependent correction factor defined in Appendix III. 
The reactivity coefficients, $\alpha_{f}, C_{f}, C_{q}$, and $C_{i}$ are defined in Section 2.3. (see also Appendix I).

The frequency response functions $F_{\text {av }}$ and $F_{S}$ are defined in Section 2.5. The frequency response functions, which are derived by Caldarola ${ }^{(6)}$, may be written as follows:

$$
\begin{aligned}
F_{s} & =\frac{2 J_{1}(x) / x}{J_{0}(x)+i \omega \gamma 2 J_{1}(x) / x} \\
F_{a v} & =\frac{1}{i \omega\left(\gamma+v^{2} / /_{8}\right)}\left[1-F_{s}\right]
\end{aligned}
$$

where $J_{0}(x)$ and $J_{1}(x)$ are Bessel functions of the first kind of order zero and order one, respectively.

The argument $x$ is defined by: $x=1 / 2$, $\sqrt{\omega}$. $(2,5-5)$ where $\omega$ is the frequency of oscillation in radians per second, and $i=\sqrt{-1}$.

The parameters $\gamma$ and $\nu$ can be expressed in terms of fuel density, $\rho$, heat capacity, $c$, average conductivity $k$, fuel pellet radius $R$, and the effective heat transfer coefficient $h_{f}$ as follows:

$$
\begin{aligned}
& \gamma=\rho \mathrm{cR} / 2 \mathrm{~h}_{\mathrm{f}} \\
& v^{2}=\rho \mathrm{cR}^{2} / \mathrm{k}
\end{aligned}
$$

From the definitions of $\mathrm{K}_{\mathrm{av}}$ and $\mathrm{K}_{\mathrm{s}}$ given in Section $2: 3.2$, it follows that:

$$
\begin{aligned}
& \mathrm{K}_{\mathrm{av}}=\frac{\mathrm{A}_{\mathrm{f}}}{\rho \mathrm{cV_{f }}}\left(\gamma+v^{2} / 8\right) \\
& \mathrm{K}_{\mathrm{S}}=\frac{\mathrm{A}_{\mathrm{f}} \gamma}{\rho \mathrm{cV_{f }}}
\end{aligned}
$$


where $A_{f}$ is the fraction of the energy which is absorbed in the fuel.

\subsubsection{Energy Coefficient}

The excess energy, $\Delta E$, that is produced as a result of a sudden power transient is defined by:

$$
\Delta E(t)=\int_{0}^{t}\left[P(u)-P_{0}\right] d y=\int_{0}^{t} \Delta P(u) d u
$$

where $P_{0}$ is the initial power level. Thus, the Fourier transform, $\Delta E(i \omega)$, of $\Delta E(t)$ is given by:

$$
\Delta E(i \omega)=\Delta P(i \omega) / i \omega
$$

Neglecting gamma and neutron heating in the coolant and structure, a reactivity energy coefficient $\gamma_{E}(c / M W-s e c-p r o d u c e d)$ can be obtained from the oscillator tests. By definition:

$$
\begin{aligned}
& \gamma_{E} \equiv \lim _{i \omega \rightarrow \infty} \frac{\Delta k_{f}(i \omega)}{\Delta E(i \omega)} \doteq \lim _{i \omega \rightarrow \infty} i \omega u_{f} F_{a v}(i \omega) x_{f}(i \omega) \\
& \gamma_{E}=\frac{\alpha_{f}}{\left(\gamma+v^{2} / 8\right)} x_{f}(\infty)=0.93 \alpha_{f} /\left(\gamma+v^{2} / 8\right)
\end{aligned}
$$

where the calculated value $\mathrm{X}_{\mathrm{f}}\left({ }^{(\infty)}=0.93\right.$ (see Appendix II) has been used.

Only the fraction $A_{f}$ of the energy is absorbed in the fuel, and as shown in Appendix II, the actual excess energy absorbed in the fuel, $\Delta E_{f}$, in the first few seconds during a transient is very nearly equal to $A_{f} X_{f}(\infty) \Delta E(t)$. 
The composite power coefficient $\alpha_{f}$ is the sum of the Doppler power coefficient, $\alpha_{D}$, and the coefficient, $C_{p}$, which represents the power dependence of the fuel and fuel-clad axial expansion. effect. Thus, the Doppler energy coefficient, $n(c / M W-s e c$ absorbed in the fuel), is given by:

$$
\eta=\gamma_{E} \cdot\left(\frac{\alpha_{D}}{\alpha_{f}}\right)\left(\frac{\Delta E}{\Delta E}\right)
$$

or in terms of "measured" and "known" quantities

$$
n=\frac{\left(\alpha_{f}-c_{p}\right)}{A_{f}\left(\gamma+v^{2} / 8\right)}
$$

From previous definitions

$$
\alpha_{D}=\left(\alpha_{f}-C_{P}\right)=C_{D} K_{a v}=\frac{C_{D} A_{f}}{\rho c v_{f}}\left(\gamma+v^{2} / 8\right)
$$

where 1t is assumed (see Appendix I) that $\mathrm{K}_{f}$ of equation (2.3-8) and $\mathrm{K}_{\mathrm{av}}$ of equation $(2.5-8)$ are the same. Thus, the Doppler energy coefficient can also be written as:

$$
n=\frac{C_{D}}{\rho c v_{f}}=\frac{A}{\rho c v_{f}^{T}}{ }_{f o}
$$

where $T_{\text {fo }}{ }^{m}$ is the effective initial fuel temperature $T_{\text {co }}+R_{f} P_{0}$ raised to the m-th power (e.g. Section 2.3$)$. 
GEAP -5576

\subsubsection{Conventional Oscillator Tests}

In the conventional oscillator tests, the transform of the feedback kernel $h(i \omega) \equiv \Delta \mathrm{K}_{f}(i \omega) / \Delta \mathrm{P}(i \omega)$ is determined from the measurement of the zero power reactivity transfer function, $G_{0}$, and the at power-reactivity transfer function, $G$, (measured at a mean power level P), by means of the following expression:

$$
h(i \omega)=\frac{1}{P}\left[\frac{1}{G_{0}(i \omega)}-\frac{1}{G(i \omega)}\right]
$$

where:

$$
\begin{aligned}
& G_{0}(i \omega) \equiv\left(\Delta P / P_{0}\right) / \Delta K_{i n} \text { - measured at zero power } i .01 \mathrm{MW} . \\
& G(i \omega) \equiv(\Delta P / P) / \Delta K_{i n} \quad \text { - measured at mean power } P
\end{aligned}
$$

and where $\Delta \mathrm{K}_{\text {in }}(\mathrm{i} w)$ ls the $\tau$ ransform of the oscillating reactivity.

The model for $h(i \omega)$ (see the previous section) is given by:

$$
h(i \omega)=\alpha_{f} F_{a v}(i \omega) x_{\Gamma}(i \omega)+\left[C_{f} F_{s}(i \omega)+C_{q}\right] \Pi(i \omega)+C_{i} U_{i}(i \omega)
$$

where:

$$
\begin{aligned}
& U(i \omega) \equiv \frac{\Delta T_{c}(i \omega)}{\Delta P(i \omega)}=F_{i}(i \omega) U_{i}(i \omega)+\left[1-F_{i}(i \omega)\right] U_{0}(i \omega) \\
& U_{i}(i \omega) \equiv \frac{\Delta T_{i}(i \omega)}{\Delta P(i \omega)}
\end{aligned}
$$


and :

$$
U_{0}(i \omega)=\frac{\Delta T_{0}(i \omega)}{\Delta P(i \omega)}
$$

The transfer functions $G, U_{i}$, and $U_{0}$ are obtained from "measured" values of $\Delta \mathrm{P}(i \omega), \Delta \mathrm{K}_{i n}(i \omega) ; \Delta \mathrm{T}_{i}(i \omega)$, and $\Delta \mathrm{T}_{j}(i \omega)$. The procedures for performing these calculations are outlined in Section 5.0.3. The transfer function between average coolant temperature and power, $U(i \omega)$, is then calculated from equation (2.5-20) using $U_{i}, U_{0}$, and calculated values of $F_{i}$. ( $F_{i}$ is defined in Appendix II.)

From the "measured" values of $h(i \omega), U(i \omega)$, and $U_{i}(i \omega)$, and the calculated values of $x_{f}(i \omega)$, the parameters $\alpha_{f}^{\prime}, C_{f}, C_{q}, C_{i}, \gamma$, and $\nu$ are determined in the manner described in Section 5.2.3.2.

The Doppler energy coefficient is calculated from equation (2.5-15) using "determined" values of $\alpha_{f}, \gamma$, and $\nu$, and using calculated values of $\mathrm{C}_{\mathrm{p}}$ and $\mathrm{A}_{\mathrm{f}}$.

If it is assumed that:

$$
\mathrm{F}_{\mathrm{av}}=\mathrm{F}_{\mathrm{s}}=\frac{1}{1+i \omega \tau}
$$

the two parameters $\gamma$ and $\nu$ are replaced by the one parameter, $\tau_{f}$. The Doppler energy coefficient is then obtained from equation (2.5-15) by replacing $\gamma+\nu^{2} / 8$ by $\tau_{f}$. 
GEAP-5576

\subsubsection{First Balanced Oscillator}

In the first balanced oscillator test, the coolant flow rate and the reactivity are both oscillated. The relative phase angle and amplitudes of the two oscillations are adjusted to hold the inlet temperature and core temperature rise constant. For this experiment, the model for $h(i \omega)$ is given as before by equation (2.5-19). If a perfect balance is. achieved, the latter two terms vanish, however, and the model for $h(i \omega)$ reduces to:

$$
h(i \omega)=\alpha_{f} F_{a v}(i \omega) X_{f}(i \omega) \quad \ddots \quad(2.5-24)
$$

Thus, for a perfect balance, only three parameters, $\gamma ; v$; and $\alpha_{f}$, need be determined in order to fit the model to the measured values of $h(i \omega)$ and calculated values of $x_{f}(i \omega)$.

In addition to power and reactivity oscillations, the fractional flow oscillation $\Delta V(i \omega) / V_{0}$ is measured during the first balanced oscillator test. This measurement allows one to determine the frequency response function $F_{S}$ and thus provides an additional check on the model.

As shown in Appendix III, the ratio of the fractional flow oscillation to the fractional power oscillation can be written as:

$$
\frac{\frac{\Delta V(i \omega)}{V}}{\frac{. u}{\Delta P(i \omega) / P}}=a_{c} X_{c}+\left(1-a_{c}\right) F_{s} X_{F}-\frac{P}{\Delta \theta}\left[\left(1+b-b F_{i}\right) U_{0}-\left(1-b \vec{F}_{i}\right) U_{i}\right]
$$


GEAP -557.6

where:

$$
\begin{aligned}
\Delta V(i \omega)= & \text { transform of the flow oscillation } \\
V_{0}= & \text { mean flow rate } \\
\mathrm{X}_{\mathrm{c}}(\mathrm{i} \omega)= & \begin{array}{l}
\text { transform of the ratio of total power absorbed } \\
\text { in the coolant and structure to the "fission" }
\end{array} \\
& \begin{array}{l}
\text { power absorbed in the coolant and structure } \\
\text { (see Appendix II) }
\end{array} \\
\Delta \theta \quad & \text { steady-state core temperature rise at power } \\
& \text { P and mean flow rate } \mathrm{V}_{0}
\end{aligned}
$$

The function $b(i \omega)$ is a calculated function defined in Appendix. III and the other quantities are as previously defined.

The latter term vanishes in the event of a perfect balance and equation $(2.5-25)$ reduces to:

$$
\frac{\Delta V / V_{o}}{\Delta P / P}=a_{c} x_{c}+\left(1-a_{c}\right) F_{s} \ddot{x}_{f}
$$

Measured quantities are $\Delta \mathrm{V} / \mathrm{V}_{\circ}$ and $\Delta \mathrm{P} / \mathrm{P}_{0^{\circ}}$ Calculated quantities are $x_{c}, a_{c}$, and $x_{f}$. The two parameters to be determined, are $\gamma$ and $v$ (the parameters in the model for $\mathrm{F}_{\mathrm{S}}$ )...

It should be noted that it has. been assumed that the frequency response function $\mathrm{F}_{\mathrm{S}}(i \omega)$ in the equation related to reactivity feedback (i.e. equation $(2.5-1),(2.5-24)$, and $(2.5-28)$ below) is the same as the function used in $(2.5-25),(2.5-26)$, and $(2.5-31)$ below). 
This assumption is strictly valid only if the fuel "thermal impedance" is constant throughout the core, since the former equations involve an "effective fuel temperature for reactivity feedback" (an importance weighted temperature) while the latter equations involve an "effective fuel temperature for heat transfer" (an "average". temperature). ...

\section{5 .5 Second Balanced Oscillator Test}

In the second balanced oscillator test, the coolant flow rate and the reactivity are both oscillated. The relative phase angle and amplitude of the two oscillations are adjusted to hold the power constant.

$$
\text { Since: } \quad \frac{\Delta P(i \omega)}{G_{0}(i \omega)}=P_{i}\left[\Delta K_{i n}(i \omega)+\Delta K_{f}(i \omega)\right]
$$

the transfer function between the reactivity oscillation, $\Delta \mathrm{K}_{i n}$, and the average conlant temperature oscillation, $\Delta T_{c}$, is given by:

$$
\frac{\Delta \mathrm{K}_{i n}}{\Delta \mathrm{T}_{\mathrm{c}}}=-\left(\mathrm{C}_{\mathrm{f}} \mathrm{F}_{\mathrm{s}}+\mathrm{C}_{\mathrm{q}}\right)-\mathrm{C}_{i} \frac{\Delta \mathrm{T}_{\mathrm{i}}}{\Delta \mathrm{T}_{\mathrm{c}}}+\left(\frac{1}{\mathrm{PG}_{0}}-\alpha_{f^{\mathrm{F}}} \mathrm{F}_{\mathrm{t}}\right) \frac{\Delta \mathrm{P}}{\Delta \mathrm{T}_{\mathrm{c}}}
$$

where, as before, $\Delta \mathrm{T}_{c}$ is defined in terms of the measure inlel and outlet temperature oscillations and the calculated factor $F_{i}$ as follows:

$$
\Delta \mathrm{T}_{c}=\mathrm{F}_{i} \Delta \mathrm{T}_{i}+\left(1-\mathrm{F}_{i}\right) \Delta \mathrm{T}_{0}
$$

. The measured quantities are $\Delta \mathrm{K}_{\text {in }}, \Delta \mathrm{T}_{1}$, and $\Delta \mathrm{T}_{0^{\circ}}$. The calculated quantity is $\mathrm{F}_{i}$ and the parameters to be determined are $\mathrm{C}_{f}, \mathrm{C}_{q}, \mathrm{C}_{i}, \gamma$, and $v$. 


\section{5 .6 Additional Measurements}

In either the conventional oscillator test or the balanced oscillator tests an additional check on the model and on the parameters $\gamma$ and $\nu$ can be obtained by analyzing the central fuel temperature response (measured by the instrumented fuel assemblies) to a power oscillation. The model for the measured transfer function is given by $(6)$

$$
\frac{\Delta T_{c f}(i \omega)}{\Delta P(i \omega)}=K_{c} F_{c}(i \omega)+G_{c} U(i \omega)
$$

where $\mathrm{K}_{\mathrm{c}}$ is the static temperature change in the center fuel temperature for a power change at constant coolant temperature and. where $U(i \omega)$ is the response of the average coolant temperature to a power oscillation as defined in Section 2.3.3.

The models for the frequency response functions, $F_{c}$ and $G_{c}$, can be defined in terms of the parameters $\gamma$ and $\nu$ (see Section 2.3.2), and the frequency response function, $F_{S}$, (see Section 2.3.2) as follows: :

$$
G_{c}(i \omega) \equiv \frac{x F_{s}}{2 J_{1}(x)}
$$

and:

$$
F \cdot(i \omega)=\frac{1}{i \omega\left(\gamma+v^{2} / 4\right)}\left[1-G_{C}\right]
$$

and where $x$ and $J_{1}(x)$ are defined in Section 2.3.2. 
GEAP -5576

Measurements of $\Delta \mathrm{T}_{\mathrm{cf}} / \Delta \mathrm{P}$ and $\mathrm{U}$ can be used with the above expression to obtain $K_{c}$ and an independent check on $\gamma$ and $v$.

\subsection{REACTIVITY MODEL FOR TRANSIENT TESTS}

\subsubsection{Doppler Effect}

The transient tests involve rapid insertion of positive reactivity in a time which is very short compared to the fuel time constant and the fuel temperature rise during the transient occurs approximately adiabatically. The change in fuel tempera- . ture, $\Delta \mathrm{T}_{\mathrm{f}}$, may thus be approximated, by:

$$
\Delta \mathrm{T}_{\mathrm{f}}=\frac{1}{\rho \mathrm{cV}} \Delta \mathrm{E}_{\mathrm{f}}
$$

where $\rho$ is the fuel density, $c$ is the fuel heat capacity, $v_{f}$ is the fuel volume, and $\Delta \mathrm{E}_{\mathrm{f}}$ is the transient energy absorbed, in the fuel.

From equations $(2.3-6)$ and $(2.6-1)$, the cransient Doppler reactivity effect may thus be written as:

$$
\Delta k_{D}=A \ln \left[1+\frac{\Delta E_{f}}{\rho c V_{f}^{T} T_{f o}}\right]
$$

where $\mathrm{T}_{\mathrm{fo}}$ is the initial effective fuel temperature at the beginning of the transient. Ulsing equation (2.5-17), w1th $n=1$, th1s can be written as:

$$
\Delta \mathrm{k}_{\mathrm{D}}=\mathrm{Aln}\left[\mathrm{l}+\frac{\mathrm{n}}{\mathrm{A}} \Delta \mathrm{E}_{\mathrm{f}}\right]
$$


or for $\frac{\eta^{\prime}}{A} \Delta E_{f} \ll 1$

$$
\Delta \mathrm{k}_{\mathrm{D}} \simeq \mathrm{n \Delta \textrm {E } _ { \mathrm { f } }}
$$

For very large transients ( $\dot{\Delta} \mathrm{E}_{\mathrm{f}} / \mathrm{A}$ not $<1$ ) the expression given by (2.6-3) or a corresponding expression, if the logarithmic model is not applicable "(see Section 2:3.1), can" in principle, be used to determine both $A$ and $n$ from analysis of transient data." For SEFOR, the quantity $n / A$ is of the order of 0.001 (MW-sec) and the planned transients will produce an energy relase of the order of $200 \mathrm{MW}-\mathrm{sec}$. Thus, the logarithmic nature of the Doppler effect is not emphasized during the transient and it is expected that the Doppler feedback can be accurately represented by a second or third order polynomial in $\Delta \mathrm{E}_{\mathrm{f}}$, even for the super-prompt critical transients. Such a representation may be written as:

$$
\Delta \dot{k}_{\mathrm{D}}=n \Delta \mathrm{E}_{\mathrm{f}} \equiv\left(\eta_{\mathrm{o}}+\dot{\eta}_{1} \Delta \mathrm{E}_{\mathrm{f}}+\ddot{\eta}_{2} \Delta \dot{\mathrm{E}}_{\mathrm{f}}^{\dot{2}}\right) \ddot{\Delta \mathrm{E}} \ddot{f}_{\mathrm{f}}
$$

\subsubsection{Non-Doppler Feedback}

In the transient tests, reactivity feedback due to increased fuel-clad and sodium-structure temperature, as well as reactivity feedback due to fuel axial expansion will occur along with the Doppler feedback. Most of the temperature rise in the sodiumstructure temperature is due to neutron and prompt gamma heating while a portion of the fuel-clad axial expansion effect and the 
GEAP -5576

fuel axial expansion effect will depend on the fuel temperature. It is expected that these non-Doppler effects will contribute only $215 \%$ of the total reactivity feedback during the first 200 to $40 \mathrm{cmsec}$ of the transient.

The model for the non-Doppler feedback, $\Delta \mathrm{K}_{\mathrm{o}}$, "is of the form

$$
\Delta K_{o}(t)=C_{e} \Delta T_{e}(t)+C_{t} \Delta T_{t}(t)+C_{c} \Delta T_{c}(t)
$$

where $\Delta \mathrm{T}_{\mathrm{e}}$ is the "fuel shoulder temperature," $\Delta \mathrm{T}_{t}$ is the average clad temperature and $\Delta \mathrm{T}_{\mathrm{c}}$ is the average coolant temperature. The coefficients $\mathrm{C}_{e}, \mathrm{C}_{t}$, and $\mathrm{C}_{c}$ are the transient-fuel-expansion coefficient, the fuelclad coefficient, and the coolant-structure coefficients, respectively. The time dependent behavior of $\Delta \mathrm{T}_{e}, \Delta \mathrm{T}_{t}$, and $\Delta \mathrm{T}_{f}$ can be calculated for each transient by using a code such as ARGUS ${ }^{(10)}$ or FORE. (11) The nonDoppler feedback $\Delta \mathrm{K}_{0}$ is then obtained using calculated and measured values of the reactivity coefficients.

\section{$2.7 \quad$ EXPERIMEN'LAL DOMIAIN}

It is desired to investigate the validity of the reactivity model and evaluate individual reactivity feedback effects; particularly the Doppler effect, over a wide range of reactor operating conditions. Permissible equilibrium operating conditions at which test data may be obtained; are determined by plant operating characteristics and limitations and safety considerations. The range of permissible equilibrium operaling conditions is called the experimental domain. 
GEAP -5576

For purposes of planning the experimental program, the experiniental domain was letermined by evaluating the operating characteristics in the reactivity-power-average core coolant temperature domain and then superimposing reasonable operating and safety related limitations on the operating characteristics. The operating characteristics were determined from reactivity coefficients listed in the Task 8, Core Design Topical Report ${ }^{(8)}$ and the Doppler reactivity effect calculated using WEDOP(12) The following coefficients were used:

$$
\begin{aligned}
& \text { Doppler: } \quad \mathrm{T} \frac{\mathrm{dk}}{\mathrm{d} T}=-.0085 \frac{\Delta \mathrm{k}}{\mathrm{k}}
\end{aligned}
$$

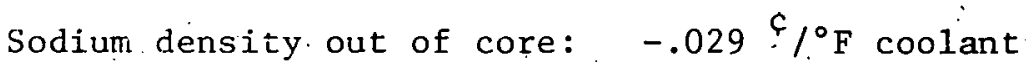

$$
\begin{aligned}
& \text { Sodium density in core: } \quad-.121 .^{6} /{ }^{\circ} \mathrm{F} \text { coolant } \\
& \text { Structure expansion: . } \quad-.233^{\mathrm{C}} /{ }^{\circ} \mathrm{F} \text { coolant } \\
& \text { BeO axial expansion: } \quad-.006 \quad \% .{ }^{\circ} \mathrm{F} \text { coolant } \\
& \text { Fuel-clad axial expansion: }-.078{ }^{\complement} / /^{\circ} \mathrm{F} \text { coolant } \\
& -.467^{\mathrm{C}} /{ }^{\circ} \mathrm{F} \text { coolant } \\
& \text { Fuel axial expansion: } \quad-.0058^{6} /{ }^{\circ} \mathrm{F} \text { fuel }
\end{aligned}
$$

Reflector temperature effects were:neglected. The, resulting operating characteristics of SEFOR are shown in Figure. 2.7-1, as the change in reactivity as a function of average coolant temper-. ature for different power levels. 


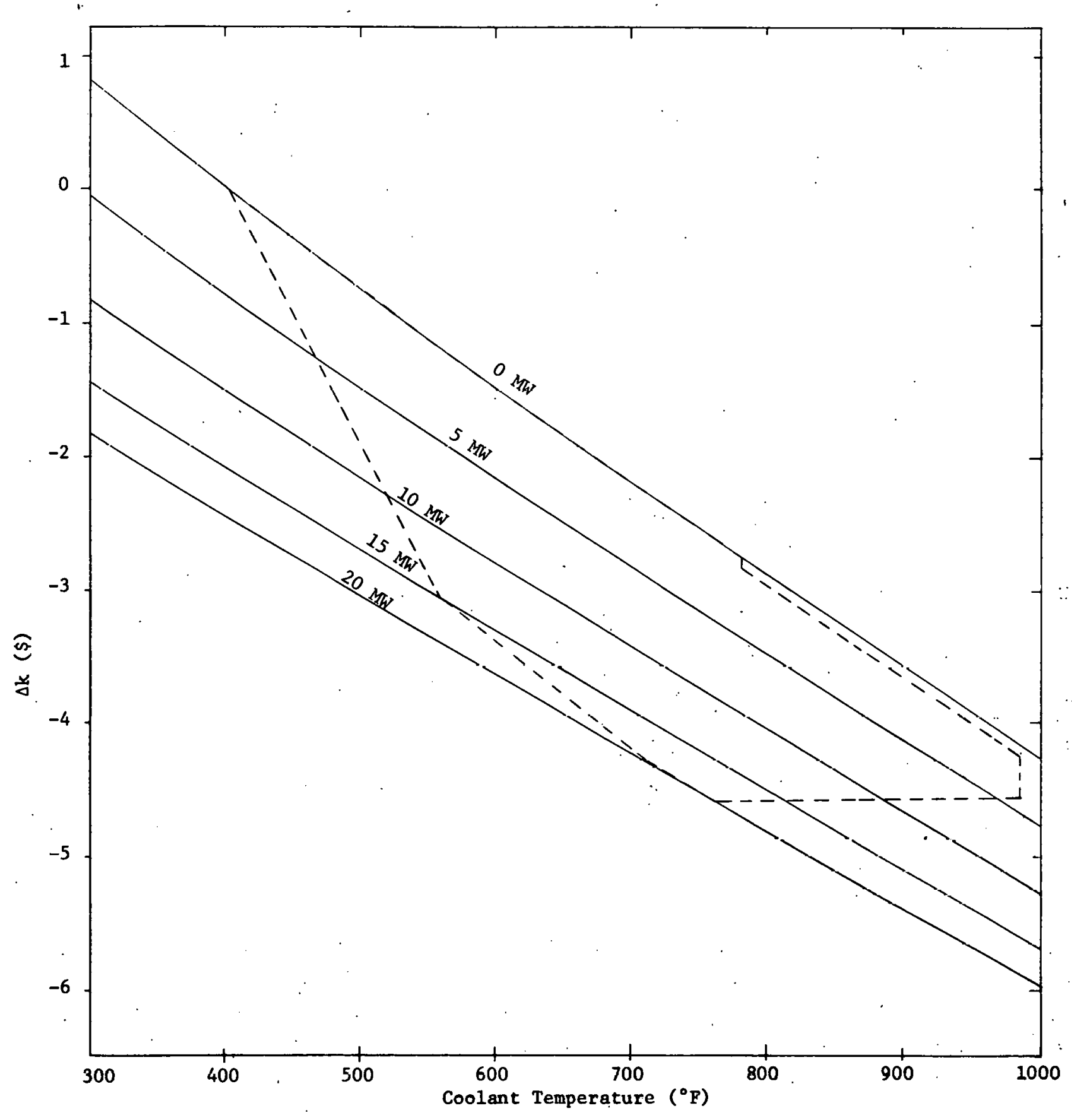

Figure 2.7-1. EXPERIMENTAL DOMAIN 
GEAP -5576

SECTION III

\section{ZERO POWER EXPERIMENTS}

3.1 INITIAL LOADING TO CRITICAL

\subsubsection{Ohjectives}

The objectives are to determine the minimum critical number of fuel rods for the reactor mode in which:

1. all reflectors are up

2. core temperature $\leq 400^{\circ} \mathrm{F}$

3. primary system contains sodium

4. nuclear power is zero

and to obcaln preliminaly estimates of reflector worth.

\subsubsection{General Procedures}

The standard method of loading fuel in which fuel is initially loaded at the center of the core structure followed by additions of fuel at the edge of the partially loaded core is recommended. Fuel should be added so as to maintain a cylindrical array of fuel that is concentric with the core structure. Although the loading increments cannot be determined before the actual test, it is expected that the minimum critical core can be achieved with 8 to 10 increments starting with an initial increment of $1 / 4$ to $1 / 3$ of the estimated minimum critical core. 
GEAP -5576

Analysis indicates that the inherent source of neutrons (due to spontaneous fission and $\alpha-n$ reactions) in SEFOR fuel will not need to be supplemented by a startup source, provided that appropriate neutron detectors are placed in the core during the critical approach. The inherent source has been estimated to be of the order of $10^{5}$ neutrons per second per fuel rod. At least two channels of instrumentation will be available for use during zero power tests, including incore neutron detectors, amplifiers, and pulse counters (scalers). Helium-3 rather than boron or fission detectors have been recommended because of their high sensitivity per unit volume, and the high temperature $\left(\sim 200^{\circ} \mathrm{C}\right)$ at which commercially available $\mathrm{He}^{3}$ chambers can be operated.

It is recommended that the detectors be housed in a drywell to provide isolation from the sodium. It may be possible to use the FRED drywell for this purpose. It appears, however, that the FRED drywell will interfere with fuel handling in adjacent channe1s; thus a special drywell will likely be provided. To maximize detector sensitivity, it should be placed in the central channel near the estimated axial power peak.

Analysis indicatès that the reactor source level neutron instrumentation will be on scale before the minimum critical core loading has been completed. Thus, it may be possible to remove the incore detectors after the critical-loading has been completed. Near the end of the critical approach, the worth of the reflectors may be approximately determined using the rod: drop technique. 
GEAP -5576

\subsubsection{Data Collection and Analysis}

Count rate data may be obtained from the scalers provided for the critical approach. Fuel loading increments are usually determined from a plot of the inverse multiplication (or inverse count rate since the count rate and multiplication are proportionally related) versus the number of fuel rods. However, in the case of SEFOR, the neutron source will be proportional. to the number of fuel rods, thus the multiplication should be proportionally related to the ratio of count rate to number of fuel rods. It is therefore recommended that the ratio, count rate/number of fuel rods, be plotted versus the number of fuel rods to analyze the critical approach loadings.

Reflector worth may be determined from rod drop data when the reactor is initially near critical by finding the prompt drop in power. If $P_{0}$ and $P_{1}$ are the pnwers hefore and after the prompt drop. respectively, then:

$$
\frac{P_{0}}{P_{1}}=1+\left|\frac{\Delta k}{\sum \beta_{i}{ }^{5}{ }_{i}}\right|=1+\left|\frac{\Delta k}{\beta}\right|
$$

where:

$$
\begin{aligned}
\xi_{i} & =\text { effectiveness of neutrons of group } i \\
\beta & =\text { effective delayed neutron fraction }
\end{aligned}
$$

This measurement of reflector worth is only expected to yicld an approximate value because of adverse effects of the transient on the spacial distribution of flux. 
GEAP-5576

Reflector worth may also be determined in terms of fuel rods if data is recorded during the critical approach with the reflectors down as wiel. as ir: the up position.

\subsubsection{Estimated Results}

The calculated effective multiplication factor as a function. of the percent of a full core that is loaded is shown in Figure 3:1-1 for all reflectors up and all reflectors down. The estimated minimum critical core is $79 \%$ (512 rods) of the full core.

The estimated worth of the reflectors at $79 \%$ of a full core is $3.5 \%: \Delta \mathrm{k} / \mathrm{k}$ (1.1\$): Or, in terms: of additional peripheral fuel rods, 94 rods:

3.2 LOADING TO FULL SIZE CORE

\section{$3 . \ddot{2} .1 \quad$ Objective}

The objective is to load additional fuel and poison rods to achieve a full size core that has enough excess reactivity to permit calibration of each fine control rod over its complete range of position.

\section{$3.2 .2 \quad$ General Procedure}

This is similar to a continuation of the critical approach except that poison rods as well as fuel rods will be loaded so as to maintain the reactivity approximately constant. To achieve a somewhat 


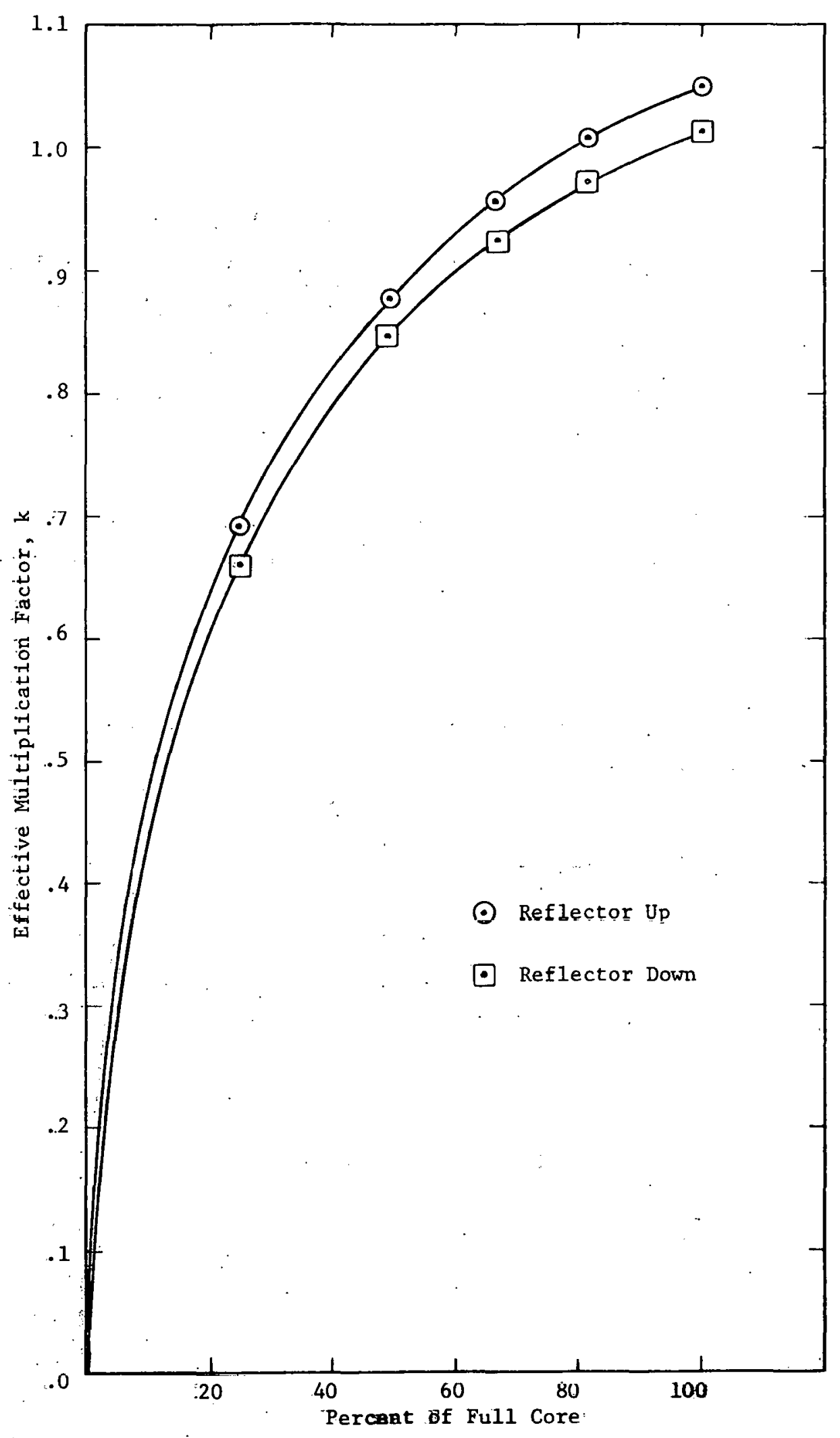

Figure 3.1-1. SEFOR GRITICAL APPROACH CENTER LOAD METHOD 
GEAP-5576

uniform distribution of poison in the core, it will be necessary to replace, with poison rods, some of the fuel rods that were loaded in the critical approach. The final loading of the core should be adjusted so that the excess reactivity allows criticality to be achieved with one fine reflector completely down, and perhaps one other reflector down slightly.

Core temperature should be held constant but the level is: not important for this step of the program. It will probably be convenient to maintain the same temperature level that is used during the approach to critical.

At various points, additional checks on the worth of the reflectors may be obtained by rod drop tests.

\subsubsection{Data Collection and Analysis}

Data collection and analysis is minimal for this step of the program. Changes in the core multiplication factor may be monttored continuously by using the low level neutron instrumentation and the initial startup detectors if they are still in the core:

\subsubsection{Estimated Resu1ts}

The worth of $\mathrm{a} \mathrm{B}_{4} \mathrm{C}$ poison rod at an average worth position in the core is $\$ 0.61$ relative to a fuel rod at that position. From Figure 3.1-1, a full core of fuel is estimated to have an excess $\mathrm{k}$ of $.05 \Delta \mathrm{k} / \mathrm{k}(15.6 \$)$ at $350^{\circ} \mathrm{F}$. With $1.5 \$$ excess for rod calibration, the worth of poison rods should be approximately $14 \$$ at $350^{\circ} \mathrm{F}$. Thus, if the loading to a full size core is carried out at $350^{\circ} \mathrm{F}$, the core will contain an èstimated 23: poison rods: 
GEAP-5576

3.3 PRELIMINARY CALIBRATION OF NEUTRON INSTRUMENTATION

\section{.3 .3 .1 Objective}

The objective is to perform an approximate calibration, in terms of reactor power level, for the reactor low level neutron instrumentation.

\subsubsection{General Procedure}

It is recommended that more than one method be used to perform a preliminary power calibration because:

a. Several inexpensive methods are available which require little or no addicional experimental time, did

b. None of the methods is expected to independently yield an accurate power calibraiton.

Procedures for four methods are given below.

Method I: This method utilizes the incore Helium-3 chambers. Power calibration of this incore instrumentation is obtained and used in turn to calibrate the low level reactor instruments. The procedure is to obtain values of the current output from the incore instruments to be calibrated, at several equilibrium power levels. The Helium-3 chambers should be located in the instrument drywell at an axial position near the fluz peak. The chambers should be operated in the pulse mode. 
GEAP -5576

Method II: This method is based on experimental determination of the output of the low level instrumentation at known values of the core multiplication factor, $k$, in the subcritical condition. Instrument calibration is then performed by using a pre-calculated relation between power and $k$.

The general procedure is to allow the core flux to achieve equilibrium at each of several subcritical conditions and record the low level instrumentation output and reflector positions.

An accurate knowledge of the source level and reactivity $\mathrm{k}$ is required for this method.

Method III: This method involves irradiation of fuel foils in SEFOR and subsequent counting of the activity of a particular fission product activity in the SEFOR counting system. It is necessary to calibrate the SEFOR counting system. The method may be applied in one or both of two different ways, depending on how the SEFOR counting system is calibrated. The SEFOR counting system may be calibrated in terms of power or terms of counter efficiency, for a particular isotope.

In the first case (method IIIa) a foil(s) (e.g. U-235 or $\mathrm{U}-238$ ) is irradiated in a flux that is similar in energy distribution to that of SEFOR, and in a position of known flux level, for a specified time interval. The foil(s) is then counted at SEFOR for a specific fission product isotope (e.g. La-140 or Mo-99) thus allowing calibration of the SEFOR counting system in terms of counting rate of a specific isotope for known reactor conditions. 
GEAP -5576

In the second case (method IIIb) an irradiated foil(s) (e.g. U-238 or U-235) is obtained that has had its activity. determined in an absolutely calibrated counter so that the total activity of a particular fission product isotope (e.g., La-140. or Mo-99) is known. The foil(s) and the activity data are then used to calibrate the SEFOR counter in counts per disintegration for the particular 1sotope chosen.

To minimize the amount of time required during the experimental program to analyze the preliminary power calibration data, the SEFOR counting system should be calibrated before the actual reactor power calibration is performed.

For either method IIIa or IIIb, the irradiation at SEFOR is made with fuel foil(s) inserted in the central drywell or in a dummy fuel rod designed for foil irradiations. To minimize the Irradiation time, the foil(s) should be located near the core flux peak. The estimated irradiation time necessary to achieve a desired foil activity can be determined from data used in method I or II of this section.

Method IV: As discussed in Section 3.11.3, the theoretical expression for the cross-power sectral density of the reactor neutron noise is:

$$
\Phi_{x y}(\omega)=\bar{q}_{x} \bar{q}_{y} F \varepsilon_{x} \varepsilon_{y} \frac{\overline{v(v-1)}}{\bar{v}^{2}} \frac{k^{2}}{\ell^{2}\left(\alpha^{2}+\omega^{2}\right)},
$$


for frequencies higher than the delayed neutron region (above $1 \mathrm{~Hz}$ ). (Symbols are defined in Section 3.11.3.). If both the average chamber current and cross-power spectral density are measured, the reactor fission rate (F) can be obtained. Thus, the above equation can be solved for the fission rate.

$$
F=\frac{\bar{i}_{x} \cdot \bar{i}_{y}}{\Phi x y} \cdot \frac{\cdots(v-1)}{\bar{v}^{2}} \cdot \frac{\mathrm{k}^{2}}{\ell^{2}\left(\alpha^{2}+\omega^{2}\right)}
$$

where $\bar{i}_{x}=F \in \bar{q}_{x}$, is the average detector current and where

$$
\therefore \frac{\overline{v(v-1)}}{\bar{v}^{2}} \approx 0.8
$$

For the case where the reactor is very near delayed critical $(1-k<\beta)$, and in the frequency range $\omega \ll \alpha$ where the power spectral density is independent of frequency, the expression for $F$ is:

$$
F=\frac{\bar{i}_{x} \cdot \bar{i}_{y}}{\Phi_{x y}(\omega)} \cdot \frac{i}{\bar{v}^{2} \beta^{2}} \quad \text { fissions } / \text { sec }
$$

This method has been checked against the foil activation technique of determining reactor power, and the two methods were found to agree within $20 \%$ at power levels between io watts and $100 \mu \mathrm{w}$ in the STARK assembly. (1) 
GEAP. -5576

\subsubsection{Data Collection and Analysis}

Method I: The following data are required: $S$, Incore chamber sensitivity, counts/sec-nv. If $S$ is not known it may be determined approximately from the relation, $S=\Sigma_{n-p} V_{c}$ where:

$$
\begin{aligned}
\mathrm{V}_{\mathrm{c}}= & \text { active volume of chamber } \\
\Gamma_{\mathrm{n}-\mathrm{p}=} & \mathrm{n}-\mathrm{p} \text { reaction macroscopic cross section for He-3, } \\
& \text { averaged over the calculated neutron spectrum at } \\
& \text { the chamber location. A value of } 3.3 \text { barns was } \\
& \text { obtained for } \sigma_{n-p} \text { for the neutron spectrum in the } \\
& \text { central channel that was calculated in core design } \\
& \text { problem C4HR1. This spectrum is given in Table. } 3-1 .
\end{aligned}
$$

Issing the additional notation:

$$
\begin{aligned}
& e \text {, dead-time correction factor ( } e \leq 1.0) \\
& R \text {, ratio of flux at the } \mathrm{He}-3 \text { chamber to the average } \\
& \text { flux in the full core } \\
& \Gamma_{\mathrm{f}} \text {, core volume and spectrum averaged fission cross } \\
& \text { section, } \mathrm{cm}^{-1} \\
& \mathrm{~V} \text {, total volume of core, } \mathrm{cm}^{3} \\
& \text { c, powèr per undt:flsslun rule, } \frac{\text { watt }}{\text { fission/sec }}
\end{aligned}
$$

The He-3 chamber may then be calibrated at a specific position with the expression,

$$
\frac{\sum_{f} V c}{R S P} \quad \frac{\text { watt }}{\text { nhserved cts/sec }}
$$




\section{GEAP -5576}

TABLE $3-1$

Calculated Neutron Spectrum in FRED Channe1 (Case C4HR1)

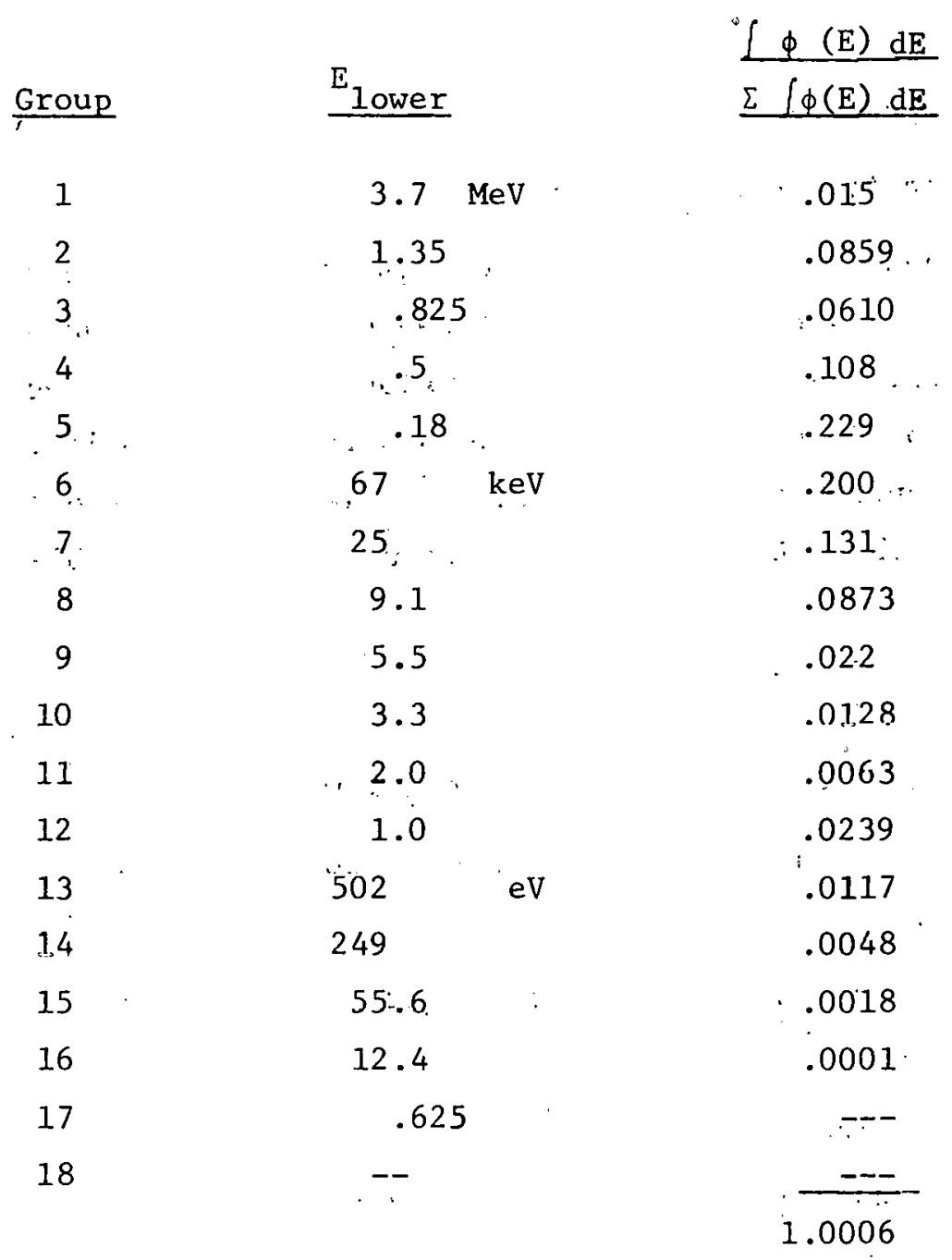


GEAP -5576

Experimental data which relates the observed counts/sec from the incore instruments to the output of the low level neutron. instrumentation can then be used with (3-2) to calibrate the low levei instrumentation.

Method II: At each subcritical condition, the output of the low level instrumentation is related to $\mathrm{k}$. By using the preliminary worths of the reflectors, as determined in 3.1 and 3.2 , or by using the calculated worths from core design data,calibration of the low level instruments can be performed. The relation between power and reactivity may be formulated as follows.

The inherent source of neutrons $\left(\mathrm{S}_{0}\right)$ ' in SEFOR is almost totally due to spontaneous fission of $\mathrm{Pu}-240$ and alpha-neutron reactions in oxygen from $\mathrm{Pu}-239$ and $\mathrm{Pu}-240$ alpha decay. Thus:

$$
S_{0}=s_{\text {s.f. }} 240 M_{240}+3_{\alpha-n} 239 M_{239}+s_{\alpha-n} 240 M_{240}
$$

where:

$$
\begin{aligned}
& s_{\text {s.t. } 240} \equiv \text { specific spontancous fission source } \\
& \text { from } \mathrm{Pu}-240=10^{6} \mathrm{n} / \mathrm{sec}-\mathrm{kg} \text { of } \mathrm{Pu}-240
\end{aligned}
$$

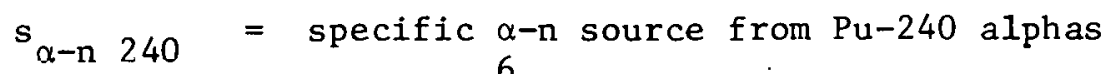

$$
\begin{aligned}
& \approx 0.29 \times 10^{6} \mathrm{n} / \mathrm{sec}-\mathrm{kg} \text { of } \mathrm{Pu}-240 \\
& s_{\alpha-n} 239=\text { specific } \alpha-n \text { source from Pu-239 alplias } \\
& \approx 0.078 \times 10^{6} \mathrm{n} / \mathrm{sec}-\mathrm{kg} \text { of } \mathrm{Pu}-239 \\
& \mathrm{M}_{240} \text { and } \mathrm{M}_{239}=\text { mass of } \mathrm{Pu}-240 \text { and } \mathrm{Pu}-239 \text {, respectively, } \\
& \text { in the core, in } \mathrm{kg} \text {. }
\end{aligned}
$$


Then for ai equilibrium subretical condition the power can be related to the multiplication factor by:

$$
P=\frac{S_{0}}{1-k} \quad \frac{k}{v} \quad c
$$

where:

$$
\begin{aligned}
& c=\text { power per unit fission rate, } \frac{\text { watt }}{\text { fission/sec }} \\
& v=\text { average number of neutrons released per fission. }
\end{aligned}
$$

In Section 3.5.4, the value of $\mathrm{S}_{\mathrm{o}} \mathrm{c} / \mathrm{v}$ is estimated to be. 0.001 watt $-\Delta k / k$, or 32 watt-cents.

Method IIIa: Consider the irradiation of a foil that contains $\mathrm{N}_{S}$ fissionable atoms, at position $x_{0}$ in a reactor. For irradiation times short enough that we can neglect destruction of any of the atoms, the rate of formation, $A$, of a particular fission product isotoped (with yield $Y$ ) of interest is:

$$
A=\int d E N_{S} \sigma_{f s}(E)^{\prime} \phi\left(E, X_{o}\right) Y \text { nuclei/sec }
$$

For energy and spatial separation of the flux $\phi$

$$
\ddot{\phi}(E, X)=F(E) \ddot{\theta}(X)
$$

thus:

$$
A=Y \dot{\theta}\left(X_{0}\right) N_{S}^{2} \int d E \sigma_{f s}(E) F(E)
$$


Then if foils of the same material are irradiated in SEFOR and another reactor, denoted by subscript 2 , having similar flux spectrum $F(E)$, the ratio of activation rates in the two reactors is :

$$
\frac{A_{\text {SEFOR }}}{A_{2}}=\frac{\theta\left(X_{0}\right)_{\text {SEFOR }}}{N_{S} \text { SEFOR }}
$$

The total power, $\mathrm{P}$, generated in a reactor with volume $\mathrm{V}$ and number of fuel atoms $\mathrm{N}_{f}$, may be represented by:

$$
P=c \int d E \int d V \sigma_{f}(E) \frac{N_{f}}{V} \quad F(E) \theta(X)
$$

For SEFOR and another reactor (or critical assembly), with a similar flux spectrum and fuel,

$$
\left(\frac{\mathrm{P}}{\mathrm{N}_{\mathrm{f}} \bar{\theta}}\right)_{\mathrm{SEFOR}}=\left(\frac{\mathrm{P}}{\mathrm{N}_{\mathrm{f}} \bar{\theta}}\right)_{2}
$$

Since the flux at the sample location is related to the total power, equation (3-8) can be written as:

$$
\frac{\mathrm{A}_{\text {SEFOR }}}{\mathrm{A}_{2}}=\frac{\mathrm{P}_{\text {SEFOR }} \mathrm{N}_{\mathrm{f} 2} \mathrm{~N}_{\text {SSEFOR }}}{\mathrm{P}_{2}}\left(\frac{\theta\left(\mathrm{X}_{\mathrm{O}}\right)}{\bar{\theta}}\right) \underset{\text { SEFFOR }}{\mathrm{N}_{\mathrm{S} 2}}\left(\frac{\bar{\theta}}{\theta\left(\mathrm{X}_{\mathrm{o}}\right)}\right)_{2}
$$


The foil activity at the time cf counting is related to tive activation rate by a function which depends on irradiation time, $t_{i}$, and decay time after irradiation, $t_{d}$. In particular, if we neglect absorption removal of the product isotope of interest, then the activity, a(dis/sec), at time, ${ }^{t}$, is :

$$
a=A\left(I-e^{-\lambda t} i\right) e^{-\lambda t} d \quad \text { dis/sec }
$$

Thus, the SEFOR power can be found from equations (3-11) and $(3-12)$ :

$$
\begin{aligned}
P_{S E F O R}= & \frac{P_{2} N_{\text {fSEFOR }} N_{S 2}}{N_{f 2} N_{S S E F O R}}\left(\frac{\theta\left(x_{o}\right)}{\bar{\theta}}\right)_{2}\left(\frac{\bar{\theta}}{\theta\left(x_{o}\right)}\right)_{\text {SEFOR }} x \\
& \frac{a_{\text {SEFOR }}\left[\left(1-e^{-\lambda t} i\right) e^{-\lambda t} d\right]_{2}}{a_{2}\left[\left(1-e^{-\lambda t} i\right) \cdot e^{-\lambda t} d\right] \text { SEFOR }}
\end{aligned}
$$

If the type of fuel and the flux spectrum, $F(E)$, in reactor-2- is not similar to SEFOR, equation (3-10) is invalid, and the right-hand side of equation (3-13) must be multiplied by the following correction factor:

$$
\left(\frac{\overline{\sigma_{f S}}}{\bar{\sigma}_{f}(\text { fue1) }}\right)_{2} \quad\left(\frac{\bar{\sigma}_{f} \text { fue1) }}{\overline{\sigma_{f s}}}\right)_{S E F O R}
$$

where the "bar" over the fission cross section denotes flux spectrum averaged cross sections. 
Method IIIb: The power generation in SEFOR may be expressed as:

$$
P=c \iint E^{\prime} \int d V \sigma_{f}(E) \frac{N_{f}}{V} \phi(E, X) \text { watts }
$$

Assuming that $\phi(E, X)=F(E) \theta(X)$ gives:

$$
P=\mathrm{cN}_{f} \int \mathrm{dE} \sigma_{f}(\mathrm{E}) \mathrm{F}(\mathrm{E}) \quad \frac{\int \mathrm{dV} \theta(\mathrm{X})}{\mathrm{V}}=\mathrm{cN}_{\mathrm{f}} \dot{\bar{\sigma}}_{\mathrm{f}} \overline{\bar{\theta}}
$$

The rate of formation, $A$, of a particular fission product isotope in a fuel foil at position $x_{0}$ is:

$$
A=Y N_{s} \int d E \sigma_{f s}(E) \phi\left(E, X_{o}\right) \quad \text { nuclei/sec }
$$

The activity, a, of this fission product isotope (with decay constant $\lambda$ ) in the foil, after irradiation for time, $t_{i}$, and subsequent decay for time, $t_{d}$, is:

$$
a=A\left(1-e^{-\lambda t} i\right) e^{-\lambda t} d \quad \text { dis/sec }
$$

Thus:

$$
\begin{aligned}
& a=\mathrm{YN}_{S}\left(1-e^{-\lambda t} 1\right) e^{-\lambda t} d \quad \int d E \sigma_{f s}(E) F(E) \theta\left(X_{o}\right) \\
& a=Y_{S}\left(1-e^{-\lambda t} i\right) e^{-\lambda t} d \bar{\sigma}_{f s} \theta\left(X_{0}\right) \text { dis/sec }
\end{aligned}
$$

Combining $(3-18)$ and $(3-15)$ we get:

$$
a=\frac{Y_{s} P \bar{\sigma}_{f s \theta(X o)}}{c N_{f} \bar{\sigma}_{f} \bar{\theta}}\left(1-e^{-\lambda t} i\right) e^{-\lambda t} d
$$


GEAP -5576

Then if the calibration factor of the SEFOK counting system for the fission product activity of interest is measured to be $\alpha$ counts/disintegration (so that a observed $=a \alpha$ ), the power level of SEFOR may be determined from:

$$
P=\frac{\left(a_{\text {observed }}\right) \mathrm{cN}_{f} \cdot \bar{\sigma}_{f} \bar{\theta}}{\alpha \mathrm{YN}_{S} \bar{\sigma}_{f s}\left(\mathrm{X}_{\mathrm{o}}\right) \mathrm{e}^{-\lambda t} \mathrm{~d}\left(\mathrm{I}-\mathrm{e}^{-\lambda t} i\right)} \text { watts }
$$

\subsubsection{Estimated Results}

The calculated calibration of the low level neutron instrumentation is 3000 counts/sec-watt. This is based on values of the neutron flux in the location of the low level sensors, from the SEFOR shielding analysis and on an estimated sensor sensitivity of 2 counts/sec per equivalent thermal neitron $/ \mathrm{cm}^{2}-\mathrm{sec}$ at the sensor. It is estimated that the calculated calibration may be error by up to a factor of five.

Based on data from noise experiments in the SEFOR loading of ZPR-JII ${ }^{(2)}$ a calibration factor of $10^{6}$ counts/sec-watt is estimated for a Helium-3 chamber with the characteristics listed below, located near the center of the core.

$$
\begin{array}{ll}
\text { Chamber length } & \sim 6 \text { inches } \\
\text { Chamber diameter } & \sim 1 \text { inch } \\
\text { Gas pressure. } & \sim \text { 10 atmospheres }
\end{array}
$$


GEAP -5576

3.4 PRELIMINARY ISOTHERMAL TEMPERATURE COEFFICIENT

\section{4:1 Objective}

The objective of this test is to: obtain preliminary. Information concerning the isothermal temperature coefficient of the core.. This information would be used when determining an appropriate fuel loading for normal operating temperatures and for preliminary correction of data from other zero power tests for undesired changes in core temperature.

\subsubsection{General Procedure}

Establish criticality at the zero power reference temperature of $\sim 400^{\circ} \mathrm{F}$ with heat supplied to the :coolant by the electrical heaters. Allow the system to achieve nuclear and thermal equilibrium at a convenient flux level. Using the electrical heaters raise the core temperature $\sim 50^{\circ} \mathrm{F}$ while maintaing a constant flux level by raising a fine reflector(s): Allow the system to achieve thermal equilibrium at the higher temperature.

After recording appropriate data reverse the procedure by lowering the core temperature $\sim 50^{\circ} \mathrm{F}$ while maintaining a constant flux level, and re-establish thermal equilibrium. 
GEAP -5576

\subsubsection{Data Collection and Analysis}

System parameters should be recorded before and after (and possibly during) each of the two temperature changes. Recorded parameters should include the time, core inlet and outlet coolant temperatures, coolant flow., fine "reflectór position(s); selected fuel temperatures, and relative flux or power level. It may be. necessary to record the parameters several times at each temperature. level to ensure that an equilibrium condition has been established..

Since the reflectors may not be accurately calibrated at this point in the experimental program it may be necessary to use the calculated reflector worth data, possibly modified by the results of reflector worths determined in Section 3.1, to determine. a preliminary value for the isothermal temperature coefficient. If readings are.taken of $T_{1}$ and $T_{2}$, the core temperatures for conditions 1 and 2 , respectively, the preliminary isothermal temperature coefficient may: be calculated simply, by dividing the change.in reactivity by the change, in isothermal temperature.

\subsubsection{Estimated Results}

The isothermal temperature coefficient may be estimated from the data given in Section 2.4. For example, at an isothermal temperature of $400^{\circ} \mathrm{F}$, the coefficient is estimated to be $-.79 \mathrm{c} /{ }^{\circ} \mathrm{F}$. 


\subsubsection{Objective}

The objective of this experiment is to determine a power level such that the presence of the inherent neutron source will have a negligible effect on the results of zero power experiments. (e.g., reflector calibrations) performed at or above that level.

\subsubsection{General Procedure}

The general procedure to be followed is to establish a series of steady-state conditions (at successively higher power levels) starting from a power level of less than $\sim$ one watt. The position of a fine reflector is noted at each of the steady-state power levels. At higher power levels, the inherent neutron source becomes lcas cignificant relative to the source from multiplication, thus the steady-state $k_{\text {eff }}$ asymptotically approaches unity with increasing power level. The deviation from criticality, at steady-state, can be made arbitrarily small.

Practically, the source effect is sufficiently small when the power is high enough so that the equilibrlum change in reflector position for a decade change in power level is small compared to the minimum possible detectable change. 


$$
\text { GEAP }-5576
$$

\subsubsection{Data Collection and Analysis}

At each steady-state condition, the power level, reflector, position, and core coolant temperature are determined. Data from the preliminary measurement of the isothermal temperature coefficient may be used to correct the reflector positions for changes in core temperature.

It is suggested that the data be analyzed by plotting the reflector position as a function of steady-state power level, or reciprocal steady-state power as a function of reflector position.
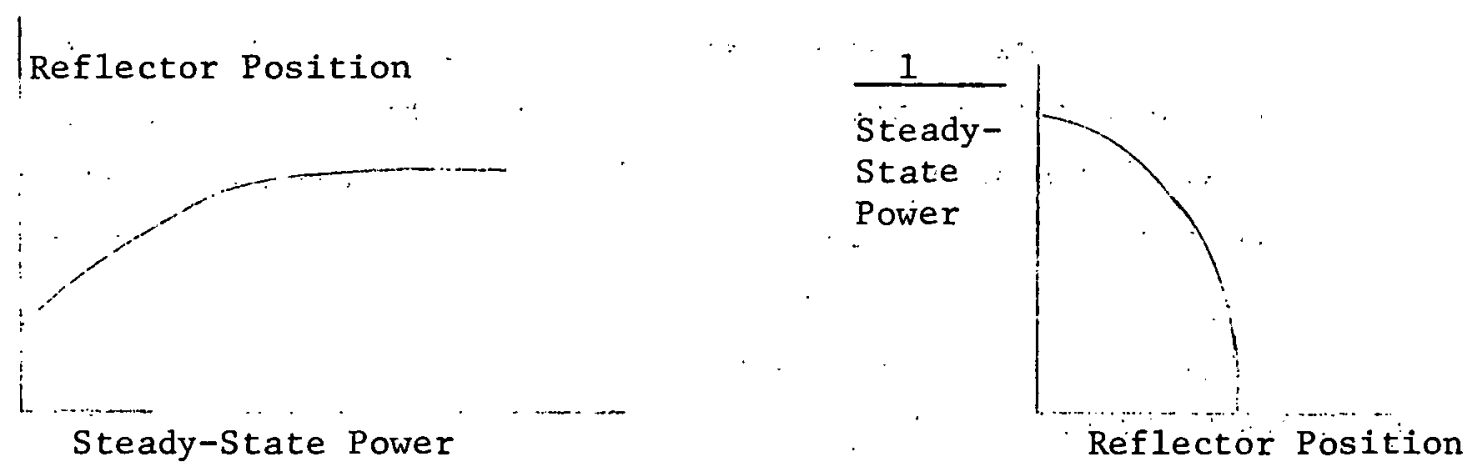

\subsubsection{Estimated Results}

The estimated inherent neutron source due to spontaneous fission of $\mathrm{Pu}-2340$ and $\alpha-n$ reactions in oxide fuel from $\mathrm{Pu}-240^{\circ}$ and $\mathrm{Pu}-239$ decay is:

$$
\begin{aligned}
& 1.3 \times 10^{6} \mathrm{n} / \mathrm{sec}-\mathrm{kg} \mathrm{Pu}-240 \\
& 7.9 \times 10^{4} \mathrm{n} / \mathrm{sec}-\mathrm{kg} \text { of } \mathrm{Pu}-239
\end{aligned}
$$


The total source based on the fully loaded design core compcosition is estimated to be $7 \times 1.0^{7} \mathrm{n} / \mathrm{sec}$. This results in an estimated source factor $C$ in the equation $P=C k / 1-k$ of:

$$
.001 \text { watt }-\frac{\Delta k}{k}
$$

or

$$
32 \text { watt }-c
$$

Thus, to reduce the source reactivity effect to less than 0.16 would require a power level of more than 320 watts.

\subsection{REFLECTOR CALIBRATION}

\subsubsection{Objective}

To obtaln the reactivily wullh of each reflector segment as a function of its vertical position. Calibrations must be obtained. with sufficient accuracy to allow accurate determination of the.. reactivity status of the reactor throughout the experimental program.

\subsubsection{General Procedure}

Each of the two fine reflectors should be calibrated differentially using the period technique and the eight. course reflectors may be calibrated by comparison to one of the calibrated fine rods using the rod swap technique. 
GEAP -5576

For period calibrations of the fine rods, reactivity increments. of approximately $10 \mathrm{c}$ are recommended. 'Such increments will result in asymptotic periods of approximately 100 seconds. The waiting times for 1 and 5 percent accuracy are approximately $100 \mathrm{sec}$ and $70 \mathrm{sec}$, respectively, if the reactor is initially critical. If the reactor is slightly subcritical in its initial state, these times may be considerably lengthened. (3) Calibrations "should be performed with an equilibrium core temperature of 350 to $400^{\circ} \mathrm{F}$ and also at approximately $760^{\circ} \mathrm{F}$, the design full power temperature. The resulting data will indicate the degree of dependence' of reflector worth on reactor temperature.

It may be advantageous to perform alternately positive and negative period measurements.

At this point in the experimental program, the core will have excess reactivity approximately equal to one reflector. The reflector calibrations will thus be representative of a 15 to 20 MW configuration. In order to perform reflector calibrations' with several different reflector: system configurations, it will be-necessary to provide additional core reactivity. To determine the effect of different reflector system configurations, reflector calibrations should be repeated after fuel loading increments are added later in the program when the core loading for high power operation is completed. If the high power core loading is completed in three equal increments, the reflector configuration after the first, second and third increments will be representative of reflector configurations for approximately 10,5 , and $0 \mathrm{MW}$ operation, respectively. 


\subsubsection{Data Collection and Analysis}

Reflector positions and the relative reactor power as a function of time are the variables of primary interest and are obtained from the normal installed instrumentation. The reactor period may be determined directly from the slope of the log power versus time curve. The corresponding reactivity may then be determined in the usual manner using the reactor lifetime and the standard reactor period-reactivity relation.

Additionally, reactor parameters normally recorded for. static tests should be recorded:

\subsubsection{Expected Results.}

The total reflector system worth was calculated to be $11 \$$. The worth of one reflector is expected to be $1.6 \$$ with all other reflectors raised and $0.8 \$$ with all other reflectors lowered. The expected rclative worth curve for the reflectors is shown in Figure 3.6.1.

\subsection{MATERIAL WÜRTH MEASUREMENTS}

\section{$3.7 .1 \quad$ Objective}

To determine the reactivity worth of $\mathrm{B}_{4} \mathrm{C}$, steel and fuel rods at various positions in the core. 
GEAP -5576

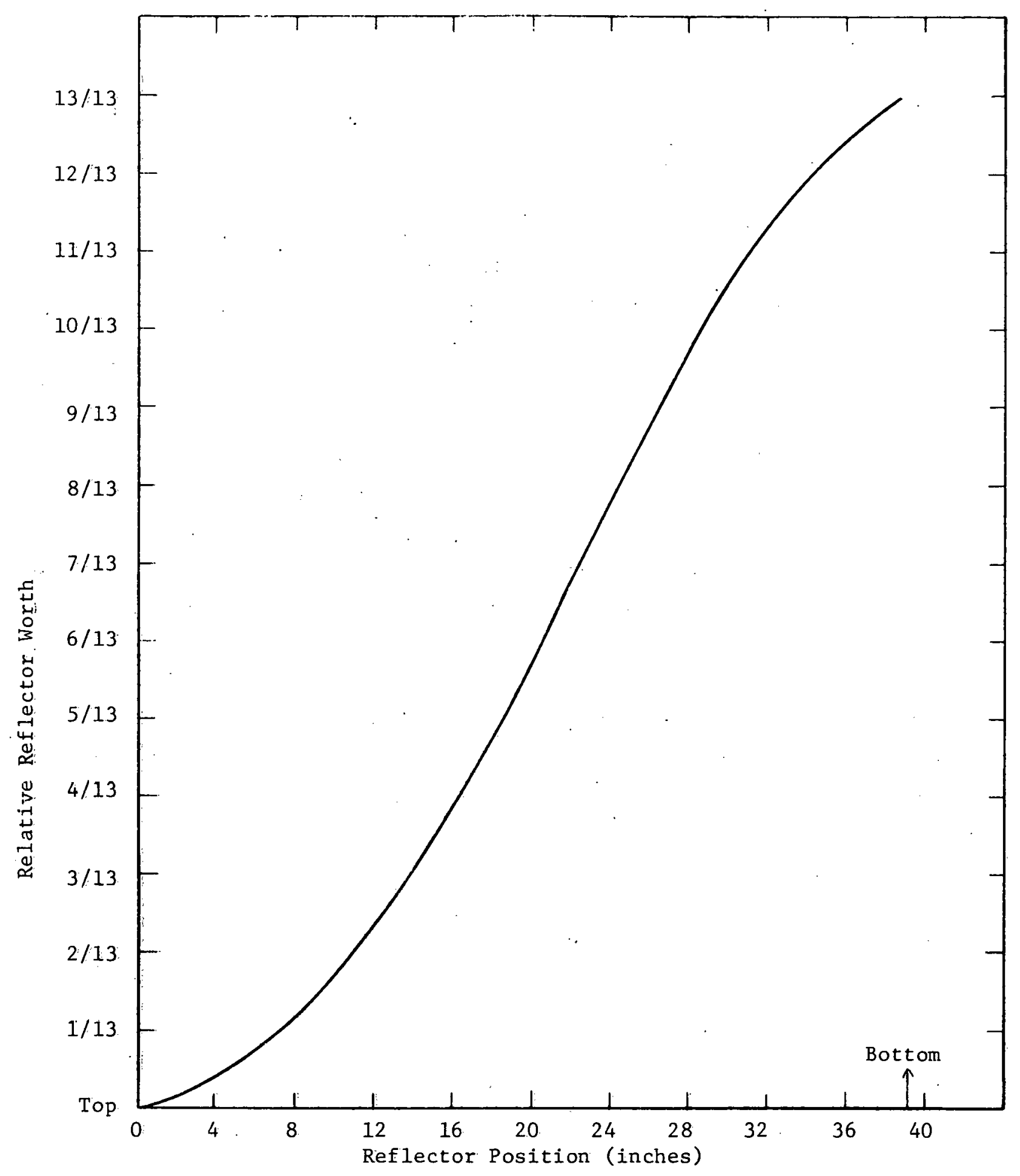

Figure 3.6-1. REFLECTOR WORTH VS. POSITION 
GEAP -5576

\subsubsection{General Procedure}

In addition to fuel rods, the plant equipment includes steel rods and rods that contain $B_{4} C$. Both types of rods are physically capable of replacing fuel rods in the core structure. The worth,. relative to sodium, of each of these three types of rods is to be determined for three radial core positions.

The reactor should be brought to nuclear and thermal equilibrium at "zero" power and a convenient core temperature. Appropriate steady-state parameters are recorded, including reflector positions, temperatures, flows and power level. At a selected core position, the fuel rod is removed and the reactivity effect is compensated by a change in fine reflector position. Similarly, the reactivity changes resulting from series placement of a steel rod and $\mathrm{a}_{4} \mathrm{C}$ rod at the same core position are compensated by changes of fine reflector position. Appropriate steady-state parameters are recorded before and after rod insertions and removals. After measurements with all types of rods are completed at a particular core position, return of the rod that originally occupied that position will affort a means of checking that the final reactivity status is identical with the original status.

Similar measurements are to be carried out for at least two additional core positions.

In preparing for these measurements, the system reactivity status should be adjusted so that reflector positions are appropriate for expected reactivity changes. 
In the selection of core positions, appropriate consideration should be given to minimizing the effects of 1) asymetrical flux distribution, for example, resulting from asymetrical reflector patterns or asymetrical core loading, and 2) local flux perturbations, for example, resulting from localized effects of the absence of regular fuel rods. It may. be necessary to choose core positions that lie along a radius that is perpendicular to the radius on which compensating reflectors are located.

In instances where it may be necessary to use more than one rod of a-given type appropriate normalization of the "identical" rods should be carried out for at least one core position.

\subsubsection{Data Collection and Analysis}

Primary data for the measurements comes from reflector positions before and after rod changes in the core. Associated reactivity changes may be determined from reflector reactivityposition calibrations. Recording of other steady-state parameters throughout these measurements will yield secondary data which can be used to perform appropriate corrections to the primary results. For example, corrections can be performed for changes in flow, temperatures and pressures that may occur during the measurements.

\subsubsection{Estimated Results}

The calculated worth of a regular fuel rod, reiative to sodium, at the center of the core is $0.39 \$$. The average worth of a fuel (relative to sodium) for all core positions except for the outer row of rods is calculated to be $0.24 \$$.

Analogous values for $\mathrm{a}_{4} \mathrm{C} \operatorname{rod}$ are $0.70 \$$ and $0.37 \$$. 
GEAP -5576

The worth of a steel rod, relative to sodium, at the center of the core is estimated to be of the order of $0.05 \$$.

These estimates are for the condition where adjacent fuel positions are occupied by regular fuel rods. Also for these estimates, it was assumed that the reflector system was in the raised position.

3.8 FLOW AND PRESSURE COEFFICIENTS OF REACTIVITY

\subsubsection{Objective}

To determine the reactivity effects of changes in primary coolant flow rate and reactor vessel hydraulic pressure.

\subsubsection{General Procedure}

The reactor should be at nuclear and thermal equilibrium at "zero" power and at some convenient core temperature. The primary coolant flow should be changed in several increments from the minimum permissible value to full flow. Thermal equilibrium should be maintained and reactivity changes should be compensated by fine reflector position changes.

In a similar manner, the reactor vessel hydraulic pressure should be changed in several increments over its design range and any resulting reactivity effects compensated by fine reflector movement. 
GEAP -5576

\subsubsection{Data Collection and Analysis}

Normal steady-state plant data should be recorded before and after each pressure and flow change. Fine reflector calibration data will be used to relate presssure and flow to reactivity. Changes in reactor parameters other than. flow and pressure: should. be recorded and used to perform corrections to the reactivity data. After the data have been corrected, flow and pressure coefficients: may be determined directly, or if necessary, may be determined by least square fitting of the data.

\subsubsection{Expected Results}

It is expected that the pressure and flow coefficlents of reactivity will be very small, possibly negligible.

\subsection{LOADING TO FULL POWER CORE:}

\section{$3.9 .1 \quad$ Objective}

The objective to be: achieved is to load the core to enable: operation at: design power and! temperature with appropriate exces:s: reactivity. A secondary objective is to obtain reflector calibrations at intermediate core loadings. 
GEAP-5576

\subsubsection{General Procedure}

To achieve the objective it:will be necessary to add additional fuel to the core by replacing selected non-fuel rods:with fuel rods. It is likely that some rearrangement of remaining non-fuel rods will be appropriate. It is expected that excess reactivity of $\$ 0.50$ available at design power and cemperature will provide hoth reasonable safety and experimental flexibility.

The appropriate core loading can be estimated from previously determined reactivity coefficients, material worths, reflector calibrations and the estimated power coefficient of reactivity. ". Core additions would be carried out under shutdown conditions. It is likely that due to safety considerations the final core arrangement would be achieved by using more than one loading increment.

At some intermediate core loadings the reflectors shuild be recalibrated following the general procedures given earlier. This will allow determination of the effects of reflector system arrangement on individual reflector calibrations. Reflector calibrations at intermedlate cure loadinge would thus be representative of those for the final core arrangement operated at less than full power conditions.

\subsubsection{Data Collection and Analysis}

Reactivily data from each core loading increment should be utilized to predict the reactivity effect of the subsequent increment. Data collection and analysis: associated with reflector calibrations will be similar to that discussed previously in the section on reflector Ealibration. 


\subsubsection{Expected Results}

The design criticality factor of the fully fueled SEFOR core (648 fuel rods). is $k=1.035$ at $20 \mathrm{MW}$ and $760^{\circ} \mathrm{F}$ average core coolant temperature.

The calculated average worth* of a boron carbide rod (relative to a fuel rod) is $\$ 0.61$, or $0.00195 \Delta \mathrm{k} / \mathrm{k}$. Thus, the final core loading to achieve $\$ 0.50$ excess at design power and temperature is expected to consist of 631 fuel rods and 17 boron carbide rods. This assumes that the boron rods are loaded so that the average worth value is valid.

\subsection{ACTIVATION MEASUREMENTS}

\subsubsection{Objective}

To determine the absolute power density, axial power distribution; and fission ratios of. U-238, U-235, and $\mathrm{Pu}-239$.

\subsubsection{General Procedure}

The determination of absolute power density may be accomplished by using the method(s) of Section 3.3. Refer to that section for a discussion of the general procedures and data analysis.

The axial power distribution at a fuel rod position may be determined by foil activation and by fuel rod gamma scanning methods.

* Average worth excluding the outer ring of rod positions. 
GEAP -5576

In the case of foil activation experiments, hollow foil holder rods are provided. During use, each of these rods will occupy a core fuel rod position. Foils of the composition that is selected may be placed in foil holder rods at the axial locations that are chosen for this experiment. Steel pellets may be inserted into the rods as a means of positioning the foils. $\mathrm{UO}_{2}$ pellets may be placed above and below individual foils as a means of providing a reasonably representative activation neutron flux. After the foil holder rods have been filled and closed, they are placed in position in the core. The irradiation time is chosen on the basis of the type of foil material (U-235, U-238, Pu-239), the desired foil activity at the expected time of counting, and the estimated neutron flux in the region of the foils. After completion of the irradiation, the foil holder rods may be removed from the reactor and sodium cleaning may be performed as necessary.

After removing the foils from the rods, their activities may be determined using the counting and analysis equipment that is provided. Since only the power distribution is needed, only relative activities are needed. Activity of a particular fission product (e.8. La-140) or a sum of activities could be used in this experiment. The techniques and methods involved are standard and well documented.

It may be desirable to perform this experiment at one or more radial positions that are near to reflector rods that have been specially positioned so as to yield results pertaining to the effect of their position on the axial power distribution. 
GEAP -5576

After SEFOR fuel rods have received sufficient burnup, the axial power distribution may be determined from the fission product gamma activity. The general procedure, is to remove a selected fuel rod from the core and place it. in the gamma scan facility. As the fuel rod is traversed in front of the beam slit, the activity (which is proportional to the local power density) is determined by using a single or multi-channel counting system. It is expected that either gross fission product activity or counting of a specific fission product (e.g. La-140) will prove suitable. If the multichannel. system is not available for use with the gamma scan facility and it is desired to perform analysis on the basis of individual 1sotopes, the fuel rod may be traversed repetitively accompanied by appropriate gate adjustment of the single channel system.

$$
1 .
$$

For determination of fission ratios of U-238, U-235, and Pu-239, standard foil activation methods may be used. Since fuel rods of different composition are available ( $\mathrm{UO}_{2}$, regular mixed oxide, and high $\mathrm{Pu}$ content rods) another method for determining these fission ratios may be used.

If foil activations are to be made this experiment could be combined with foil activations for:power distribution by inserting. a packet of foils, rather than a single.foil, at appropriate locations in the foil holder rods. Standard techniques using the : multi-channel analyzer for counting selected fission product activities will yield the desired fission ratios.

The second method for determining fission ratios requires that fuel rods of different compositions be placed in core positions having similar neutron fluxes for irradiation. Necessary cleanup would be performed to allow removal of the rods from the refueling cell to an area of lower background radiation. The relative activity of a selected

$$
\text { 3-34 }
$$


GEAP -5576

fission product (e.g. La-140) may be determined as a function of axial position along the rods using the multi-channel system and a shielded collimator. Care should be taken to. limit the irradiation so that fuel rodiradiation levels are appropriate for removal of the rods from the refueling cell and for close handling by personne1.

\subsubsection{Data Collection and Analysis}

Standard and well documented procedures pertaining to activity analysis of radioactive materials are applicable to the experiments discussed in this section. Normal techniques for correcting for background radiation, changes in counting efficiency and decay of isotopes to be counted may be used. Random experimental errors may be expected to occur. They should be considered quantitatively by incorporating standard techniques such as repetitive counting, chi-square testing and mean square deviation analysis into the detailed experimental procedures.

Analysis of data from fuel rods of different compositions, as part of the fission ratio experiments; involves certain mathematical relations that may not be we11 documented. Therefore, some of these relations are listed below.

Consider a case where a depleted $\mathrm{UO}_{2}$ rod and a natural $\mathrm{UO}_{2}$ rod have been irradlated in the same neutron flux and the fission product to be analyzed is Ta-140 which has a $1.6 \mathrm{MeV}$ gamma emission. 
Then:

$$
C^{D}=C_{235}^{D}+C_{238}^{D}=K\left(a_{235}^{D}+a_{238}^{D}\right)
$$

where:

Superscript D stands for depleted $\mathrm{UO}_{2}$ rod ...

C. = La-140 count rate

$\mathrm{C}_{235}=\mathrm{La}-140$ count rate for $\mathrm{U}-235$ fission products

$\mathrm{C}_{238}=\mathrm{La}-1.40$ count rate for $\mathrm{U}-238$ fission products

$\mathrm{K}=$ System countịng efficiency for $1.6 \mathrm{MeV}$.gammas

$a_{235}=$ La-140 activity for U-235 fission products

$a_{238}=$ La-140 activity for U-238 fission products

The La-140 activity for U-235 fission products is:

$$
a_{235}=y_{235} N_{235} \frac{\int \sigma_{235}^{f}(E) \phi(E) d E}{\int d E} F
$$

where:

$$
\begin{aligned}
\mathrm{y}_{235} & =\mathrm{La}-140 \text { fission yield from } \mathrm{U}-235 \\
\mathrm{~N}_{235} & =\mathrm{U}-235 \text { concentration } \\
\sigma_{235}^{\mathrm{f}} & =\mathrm{U}-235 \text { fission cross section } \\
\phi & =\text { Neutron flux } \\
\mathrm{F} & =\text { Fraction of total La-1.40 remaining at counting time }
\end{aligned}
$$


and where:

$$
\mathrm{N}_{235}=\frac{\mathrm{A} \rho_{\mathrm{u}} \mathrm{f}_{235} \mathrm{~V}}{235}
$$

where:

$$
\begin{aligned}
\mathrm{A} & =\text { Avagadro's number } \\
\rho_{\mathrm{u}} & =\text { Uranium density in fuel } \\
\mathrm{f}_{235} & =\text { Isotopic U-235 fraction of uranium } \\
\mathrm{V} & =\text { Fuel volume }
\end{aligned}
$$

Using similar notation for U-238 and combining with the above relations gives:

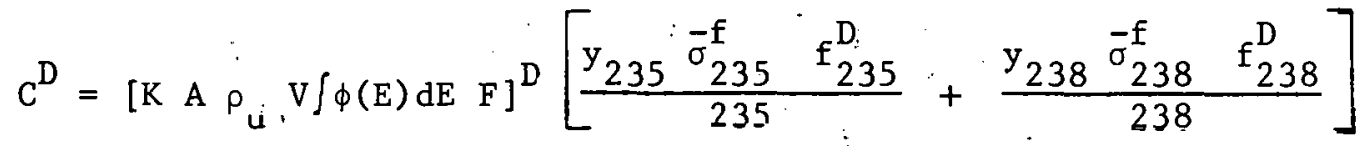

A similar relation may be:written for fuel rod that contains natural uranium which has been irradiated in the same flux (or if not, appropriate corrections performed). For this equation, the results would be corrected so that the constant term would be the same as that of equation (3-23) above. Then equation (3-23) and the analogous equation for natural uranium may be combined to yield the ratio of fission cross sections:

$$
\frac{\bar{\sigma}_{235}^{f}}{\sigma_{238}^{f}}=\frac{235}{238}\left(\frac{y_{238}}{y_{235}}\right)\left[\frac{c^{N} f_{238}^{D}-c^{D} f_{238}^{N}}{c^{D} f_{235}^{N}-c^{N} f_{235}^{D}}\right]
$$


Similarly, by including in the experiment a regular SEFOR fuel rod and a high plutonium content fuel rod, the following equations may be formed:

$$
\frac{b_{1} \sigma_{235}^{f}+b_{2} \sigma_{238}^{f f}}{b_{3} \sigma_{239}^{f f}+b_{4} \bar{\sigma}_{240}^{f}}=1
$$

$$
\frac{d_{1} \bar{\sigma}_{235}^{\mathrm{f}}+\mathrm{d}_{2} \bar{\sigma}_{238}^{\mathrm{f}}+\mathrm{d}_{2} \bar{\sigma}_{239}^{\mathrm{f}}+\mathrm{d}_{4} \bar{\sigma}_{240}^{\mathrm{f}}}{\mathrm{d}_{5} \bar{\sigma}_{235}^{\mathrm{f}}+\mathrm{d}_{6} \bar{\sigma}_{238}^{\mathrm{f}}+\mathrm{d}_{7} \bar{\sigma}_{239}^{\mathrm{f}}+\mathrm{d}_{8} \bar{\sigma}_{240}^{\mathrm{f}}}=1
$$

where the constants $a, b, c$, and are determined from known data.

Any three of equations (3-24) through :(3-27) may be combined to evaluate the desired fission cross section ratios.

\subsection{0 .4 , Expected Results}

\section{Calculated axial and radial power distributions for SEFOR} appear in Reference (4). Calculated core average cross section ratios appear in Table 3-2. These may be compared with calculated and measured values for the SEFOR critical experiment in ZPR-III. (5) 
GEAP -5576

TABLE 3-2

\section{Core Average Cross Section Ratios}

.1. Ratio

$$
\sigma_{f}(U-235) / \sigma_{f}(P u-239)
$$

$\sigma_{f}(\mathrm{Pu}-240) / \sigma_{f}(\mathrm{Pu}-239)$

$\sigma_{f}(U-238) / \sigma_{f}(\mathrm{Pu}-239)$

$\sigma_{C}(U-238) / \sigma_{f}(\mathrm{Pu}-239)$
Calculated Value

1.22

0.179

0.027

0.141

\subsection{REACTOR POWER NOISE EXPERIMENT}

\subsubsection{Objective}

To determine.the ratio of the delayed neutron fraction to the neutron lifetime, $B / \ell$.

\subsubsection{General Procedure}

The procedure follows closely that used in the noise measurements which were made in the SEFOR critical mockup in ZPR-3. (2) In the ZPR-3 measurements, the values of $\ell / \beta$ obtained by the noise and pulsed neutron methods agreed within the experiment error.(6) 
GEAP -5576

The power noise experiment should be conducted with the reactor critical, or slightly subcritical, if it should be necessary to limit the current from the neutron detectors below a certain level. The reactor should be maintained at nuclear and thermal equilibrium. Two neutron detection systems, each equipped with a Helium-3 detector, will be utilized to produce two independent reactor power noise signals. A special drywell placed in the central channel of the core should be used to house the two detectors and lead cables. The core temperature should be maintained below the detector design maximum ( $\sim 400^{\circ} \mathrm{F}$ ), or provisions for cooling the detectors should be utilized. Signal cables of appropriate design for the expected temperature should be used. It is planned that detector power supplies and signal amplifiers from the transient monitoring system will be utilized for the noise experiment. The noise signals will be recorded digitally by the equipment used for the transient neutron flux recording. To prevent aliasing of noise components that have frequencles greater than one-half of the digitizing frequency, it may be necessary to include high frequency cut-off filters in the noise detection and amplification system.

For the noise experiment, the detectors should be operated in the current mode. A supply voltage of approximately 300 volts should be satisfactory.

A separate power supply should be used in each detection system șo. that uncorrelated noise originating in. the power supplies will be eliminated by the subsequent cross correlation analysis. 
GEAP -5576

The two noise signals should be amplified so that they may be received by two transient channels of the data acquisition system. To allow analysis over the widest range of frequencies, the signals should be digitized and recorded at the maximum number of samples per second $\left(10^{4}\right)$.

Either before or after recording the reactor noise signals, the system should be operated without the neutron detectors (or-with dummy detectors) to determine the system induced noise. This data, after cross correlation analysis, can be used to correct the results from the reactor power noise analysis.

It is recommended that the Helium-3 detectors be placed side-byside at the core midplane thereby eliminating possible effects of asymmetrical axial flux distribution.

\subsection{1 .3 Data Collect1on and Analys is}

Figure 3.11-1 shows a schematic diagram of the experimental equipment used for the reactor noise measurements (similar to that used in the ZPR-3 measurements), and typical reactor operating conditions. The $\mathrm{He}^{3}$ chamber current output and efficiency have been checked in the ZPR-3 work. The RC filter network at the amplifier input blocks the d.c. chamber current slgnal and allows only the fluctuating current signal to be amplified and recorded. The RC filter passes frequencies above $1 \mathrm{~Hz}$ with no algnificant effect. A SEFOR transient amplifier can be used for these measurements since these amplifiers have greater than a $16 \mathrm{kHz}$ bandwidth with a $1000 \mathrm{pf}$ input shunt capacitance. A voltage amplifier w111 be 


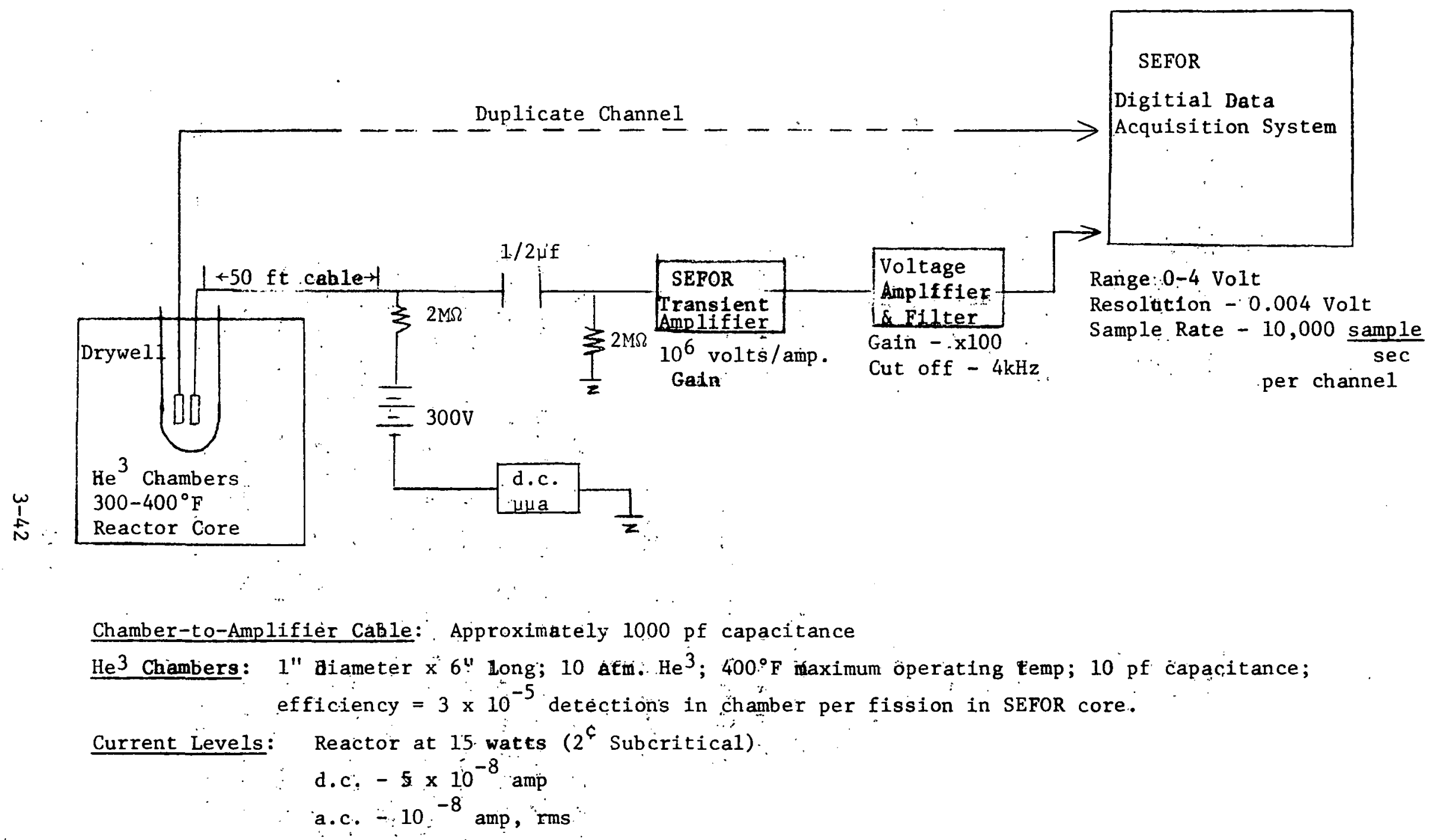

Figure 3.11-1. REACTOR POWER NOISE MEASUREMENT 
GEAP -5576

needed to raise the SEFOR transient amplifier output to a level suitable for recording. Since the sampling rate is 10,000 samples per second per channel, it will be necessary to filter out the noise above $4 \mathrm{kHz}$, in order to avoid "aliasing" (contamination of low frequencies by frequencies above $1 / 2 \times$ sample frequency). The data is sampled one channel at a time in the pattern shown below:

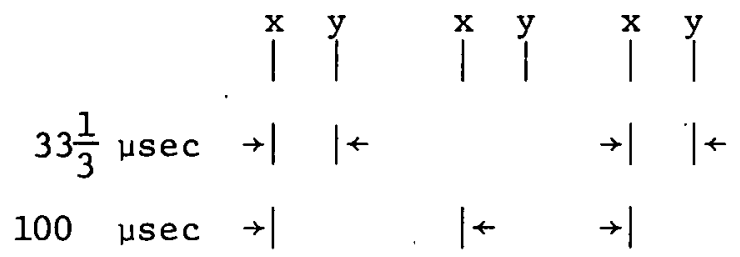

The 33-1/3 microsecond delay between sampling the two channels is not expected to significantly affect the result, since the frequency of interest is in the $1 \mathrm{kHz}$ region and the low pass filter will remove fluctuations at frequencies above $5 \mathrm{kHz}$.

There are several methods for analyzing the recorded data by digital computer. At present, it is planned to compute an estimate of the cross power spectral density $\left(\Phi_{x y}\right)$ by directly computing the Fourier transform of each channel and then computing $\Phi_{x y}$, rather than first computing the cross correlation function. Thus, the function $\Phi_{x y}$ will be estimated from:

$$
\left|\tilde{\Phi}_{\mathrm{xy}}(\omega)\right|=\frac{1}{\mathrm{~T}} \cdot \sqrt{\mathrm{A}^{2}+\mathrm{B}^{2}} \quad \frac{\mathrm{amps}^{2}-\mathrm{sec}}{\mathrm{radian}}
$$


where: $\tilde{\Phi}$ denotes the measured (estimated) value of $\Phi_{x y}(\omega)$

$$
\begin{aligned}
A & =X_{r} Y_{r}+X_{i} Y_{i} \\
B & =X_{r} Y_{i}+Y_{r} X_{i} \\
X_{r} & =\int_{0}^{T} X(t) \cos \omega t d t \\
X_{i} & =\int_{0}^{T} x(t) \sin \omega t d t \\
Y_{r} & =\int_{0}^{T} y(t) \cos \omega t d t \\
Y_{i} & =\int_{0}^{T} y(t) \sin \omega t d t
\end{aligned}
$$

where $T^{\prime}$ is the data record length in seconds. Note that $\bar{x}=\bar{y}=0$, since"the d.c. currents have been blocked by the series capacitors. The Fast Fourier transform method ${ }^{(7)}$ appears: to make this approach attractive because of shorter computer time than the alternative method described next.

Alternatively, the cross correlation function could first be computed:

$$
\tilde{\phi}_{x y}(\tau)=\frac{1}{T} \int_{x}^{T}(t) y(t+\tau) d t \text { for } 0<|\tau|<T
$$

The function $\tilde{\Phi}_{x y}(\omega)$ is then the Fourier transform of $\tilde{\phi}_{x y}(\tau)$ :

$$
\tilde{\Phi}_{x y}(\omega)=\int_{-\infty}^{\infty} \tilde{\phi}_{x y}(\tau) e^{-j \omega \tau} d \tau
$$


The function $\Phi_{x y}(\omega)$ is desired rather than $\phi_{x y}(\tau)$ since the frequency domain information gives more physical insight in the event that unexpected results occur. It is estimated that 10 to 30 seconds of data recording will be adequate to acheive an uncertainty in $\ell / B$ of a few percent. $(8)$

Prior to the reactor noise measurements, it is necessary to determine the noise background by analyzing the recorded signals with the reactor shutdown. The "background". frequency spectrum thus obtained is used to correct the reactor noise spectrum. Also, it is necessary to determine the frequency response of the data. channel so that, if necessary, corrections may be made to the reactor noise data. Of course, the $2 \mathrm{PR}-3$ work indicates that no correction for instrumentation frequency response will be necessary but a check can be made at SEFOR by using a "white noise" neutron source. A white noise neutron source can be obtained by removing the $\mathrm{He}^{3}$ chambers several feet from the reactor core and operating. the reactor at a power level high enough to give a detectable signal at the chambers.

When the $\Phi_{x y}(\omega)$ function has been determined experimentally, the value of $\ell / B$ is determined by fitting the data to the theoretical expression: (valid at frequencies above the delayed neutron region):

$$
\Phi_{x y}(\omega)=\bar{q}_{x} \bar{q}_{y} F \varepsilon_{x} \varepsilon_{y} \quad \frac{\overline{v(v-1)}}{\bar{v}^{2}} \quad \frac{k^{2}}{\ell^{2}\left(\alpha^{2}+\omega^{2}\right)}
$$

where

$$
\begin{aligned}
& \bar{q}=\text { average charge released per detected neutron } \\
& F=\text { core fission rate } \\
& \varepsilon=\text { detection in chamber per fission in core }
\end{aligned}
$$


GEAP-5576

$\nu=$ number of neutrons per fission

$$
\overline{\frac{v(v-1)}{v^{2}}} \approx 0.8
$$

$k=$ multiplication constant

$\ell=$ prompt neutron lifetime

$\ddot{\alpha}=\frac{1-k(1-\beta)}{\ell}$

$B=$ effective delayed neutron fraction

For values of $1-k<<\beta$, the expression for $\Phi_{x y}(\omega)$ reduces to:

$$
\Phi_{x y}(\omega)=\bar{q}_{x} \bar{q}_{y} F \varepsilon_{x} \varepsilon_{y} \quad \frac{\overline{v(v-1)}}{\bar{v}^{2} \beta^{2}} \quad \frac{(\beta / \ell)^{2}}{\left[(\beta / \ell)^{2}+\omega^{2}\right]}
$$

The value of $\ell / \beta$ is then found by fitting the shape of $\Phi_{x y}(\omega)$ to the function $\frac{1}{(B / \ell)^{2}+\omega^{2}}$ in the frequency range around $\omega=\beta / \ell$.

\subsubsection{Expected Results}

The expected value of $\ell / \beta$ is $2 \times 10^{-4} \mathrm{sec}$, thus the break frequency should be $800 \mathrm{~Hz}$. 
GEAP -5576

SECTION IV

STATIC POWER TESTS

\section{$4.1 \cdot$ OBJECTIVE}

The objective of the static tests conducted at power is to determine the reactivity effects associated with slow changes in reactor operating conditions over a wide range of power levels and coolant temperatures.

\subsection{GENERAL PROCEDURE}

As discussed in section 2.4 , the total reactivity feedback is a combination of several individual reactivity feedback effects. The reactivity effect of a change in the reflector temperature is easily measured experimentally. This maybe accomplished while at equilibrium power by a series of measurements in which the reflector coolant flow is changed and the corresponding reactivity effect measured. This section of the report, therefore, is primarily. concerned with the primary reactivity effects. which are related to the power level and coolant temperature.

During the static tests, the reactor is operated in an equili-. brium condition at a number of different power levels, flow rates and coolant temperatures. The tests can be performed by changing one of the reactor variables while others remain constant. The planned test conditions were.selected so that the reactivity effects associated with 1) a change in power at constant average coolant temperature, 
2) a change in average coolant temperature at constant power, and 3) a change in inlet temperature at constant power and average. coolant temperature can be. separately determined.. By. proper sequencing of the variables to be changed, a significant range of power and temperature can be covered. without a large amount of unnecessary overlap in. the measurement sequence.

The change. in reactivity, $\Delta \mathrm{k}_{\mathrm{f}}$, for a change between. two equilibrium conditions may be written as:

$$
\Delta k_{f}=A \ln \left[1+\frac{\Delta T_{c}+R_{f} \Delta P}{T_{c o}+R_{f} P_{o}}\right]+C_{p} \Delta P+C_{q} \Delta T_{c}+C_{i} \Delta T_{i}+C_{r^{\prime}} \Delta T_{r}
$$

The general procedure for conducting the static tests is to allow the reactor to achieve equilibrium conditions and then record? the values of all appropriate variables with the data acquisition. system. After the variables are recorded certain variables are changed so that the reactor achieves other desired equilibrium conditions at which the reactor variables are again recorded. This procedure is repeated until equilibrium conditions have been achieved and the- variables recorded for all desired reactor conditions.

The planned reactor equilibrium conditions for the static. tests are listed in Table:4-1. The test points that lie along lines of constant power include different coolant: flows, and. hence, different inlet coolant temperatures; for the same average coolant. temperature:. If it: is determined that the inlet. coolant temperature reactivity effect is small, it may be appropriate: to omit most of the tests that are listed for 25 and 40 percent coolant flow rates. 


\subsection{DATA COLLECTION AND ANALYSIS}

Data collection and recording will be"performed through use of the SEFOR data acquisition system. Data to be recorded includes primary coolant system temperatures, power level, primary coolant flow, reflector temperature and reflector positions. The reactivity change associated with a change.between two equilibrium reactor conditions is evaluated from the reflector data. For changes between equilibrium conditions, where the reflector temperature is the only variable that changes, equation (4-1) reduces to:

$$
\Delta \mathrm{k}_{\mathrm{f}}=\mathrm{C}_{\mathrm{r}} \Delta \mathrm{T}
$$

Similarly for static tests, in which the power and average coolant temperature do not change, we have:

$$
\Delta \mathrm{k}=\mathrm{C}_{\mathbf{i}} \Delta \mathrm{T}
$$

where the reactivity has been corrected for changes in reflector temperature, i.e.

$$
\Delta k=\Delta k_{f}-C_{r} \Delta T_{r}
$$


GEAP- 5576

TABLE 4-1

EQUILIBRIUM CONDITIONS FOR STATIC TESTS

Tests at constant average coolant temperature:

$$
\begin{aligned}
& \frac{T C}{C}=600^{\circ} \mathrm{F} \\
& \begin{array}{l}
\mathrm{P}=2,4,6,8,10^{2}, 12,14,16 \mathrm{MW} \\
\mathrm{T}=760^{\circ} \mathrm{F}
\end{array} \\
& \frac{\mathrm{C}}{\mathrm{P}=2,4,6,8,10,12,14,16,18,20 \mathrm{MW}}
\end{aligned}
$$

Tesțs at constant power:

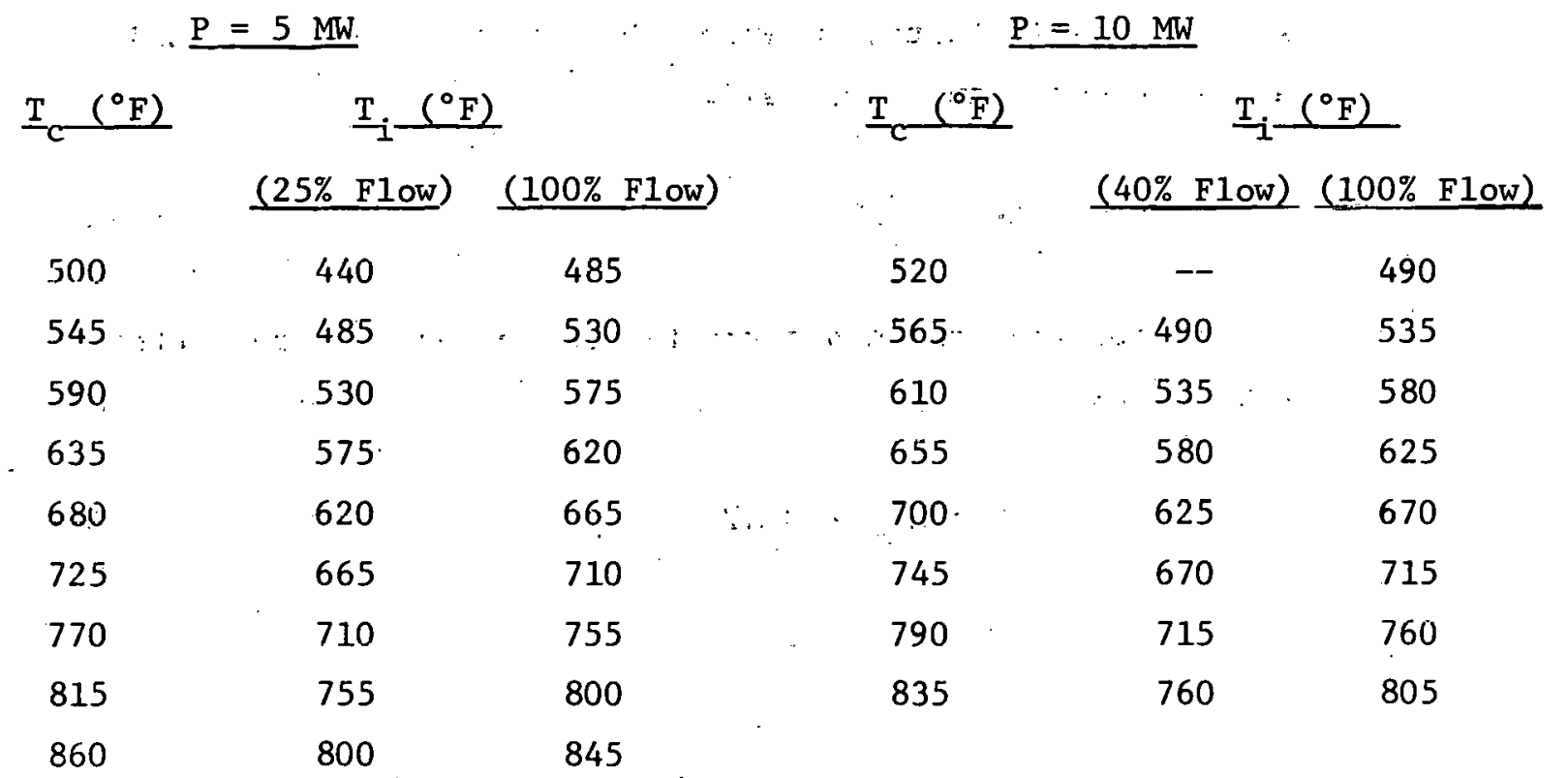


For static tests in which the average coolant temperature is held constant, we have:

$$
\Delta k=A l n\left[1+\frac{R_{f} \Delta P}{T_{c o}+R_{f} P_{o}}\right]+C_{p} \Delta P
$$

where $\Delta \mathrm{k}$ has been corrected for reflector temperature and inlet coolant temperature effects. :

For static tests in which the power is held constant, the feedback reactivity may be represented by 4-6 after correction for effects of changes in the reflector temperature and inlet coolant temperature.

$$
\Delta \mathrm{k}=\operatorname{Aln}\left[1+\frac{\Delta \mathrm{T}_{\mathrm{c}}}{\mathrm{T}_{\mathrm{co}}+\mathrm{R}_{\mathrm{f}} \mathrm{P}_{\mathrm{o}}}\right]+\mathrm{c}_{\mathrm{q}} \Delta \mathrm{T}_{\mathrm{c}}
$$

The contribution to the feedback reactivity from the Doppler effect is of primary interest in the static tests. It is expected to be the most complex contribution from the data analysis standpoint. It is recommended that models other than the simple model in equation 4-1 be considered for the Doppler reactivity contribution. Other relatively simple models for tests at constant average coolant cemperatures are:

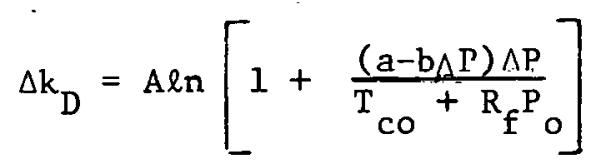

and:

$$
\Delta k_{D}=A \ln \left[1+\frac{a \Delta P}{\left(T_{c o}+R_{f} P_{o}\right)(1+b \wedge P)}\right]
$$


It is expected that least squares fitting methods will be used for reduction of the static test data to determine the best values for the reactivity coefficients. The general least squares curve fitting equations and methods to be used are presented in Appendix V.

The importance of the Doppler coefficient may require that analysis of the Doppler data be also performed using a more sophisticated model than those listêd above:

$\because$ It has been shown ${ }^{(2)}$ that the Doppler effect $\Delta \mathrm{k}_{\mathrm{D}}$ may be closely approximated by spacial weighting of the local Doppler coefficient $\mathrm{r}=\mathrm{A} / \mathrm{T}_{\mathrm{f}}^{\mathrm{m}}$ with the square of the local power density rather than the more exact flux flux-adjoint weighting. Thus, the spacially weighted Doppler reactivity effect may be represented as:

$$
\Delta k_{D}=\frac{A}{1-m} \cdot \frac{\int_{\text {fue } 1} P^{2}(r) \Delta\left[T_{f}(r)^{1-m_{j} d V}\right.}{\int_{\text {fuel }} P^{2}(r) d V} \cdot(m \neq 1)
$$

or:

$$
\Delta k_{D}=\frac{A \int_{\text {fuel }} P^{2}(r) \ln \frac{T_{f 2}(r)}{T_{f 1}(r)} d V}{\int_{\text {fuel }} P^{2}(r) d V} \quad(m=1)
$$

The compute code WEDOP ${ }^{(1)}$ has been prepared for use in evaluating the farameters $A$ and $m$ based on corrected experimental reactivity and power density distribution data and fuel thermal-hydraulics parameters. 


\subsection{ESTIMATED RESULTS}

Calculated temperature and power coefficients of reactivity at a power level of $10 \mathrm{MW}$ are listed in Table 2-1.

The calculated Doppler constant A and exponent m áre -0.0085 $\Delta k / k$ or $-2.66 \$$ and approximately 1.0 , respectively.

The estimated reactivity coefficients may be used to determine the operating characteristics under which the static tests will be performed. The calculated operating characteristics, excluding reflector and coolant flow reactivity effects are shown in Figure 4-1.

The calculated change in reactivity as a function of power, with a constant average coolant temperature of $760^{\circ} \mathrm{F}$, is shown in Figure 4-2 for WEDOP results. In addition, the best least squares fitting of the non-spatially weighted Doppler model

$$
\ln \left(1+\frac{R_{f}^{P}}{T_{c o}+R_{f} P_{o}}\right)
$$

is shown for purposes of comparison. The best fit coefficiente of three non-spatially weighted models are lișted in Table 4-2. 


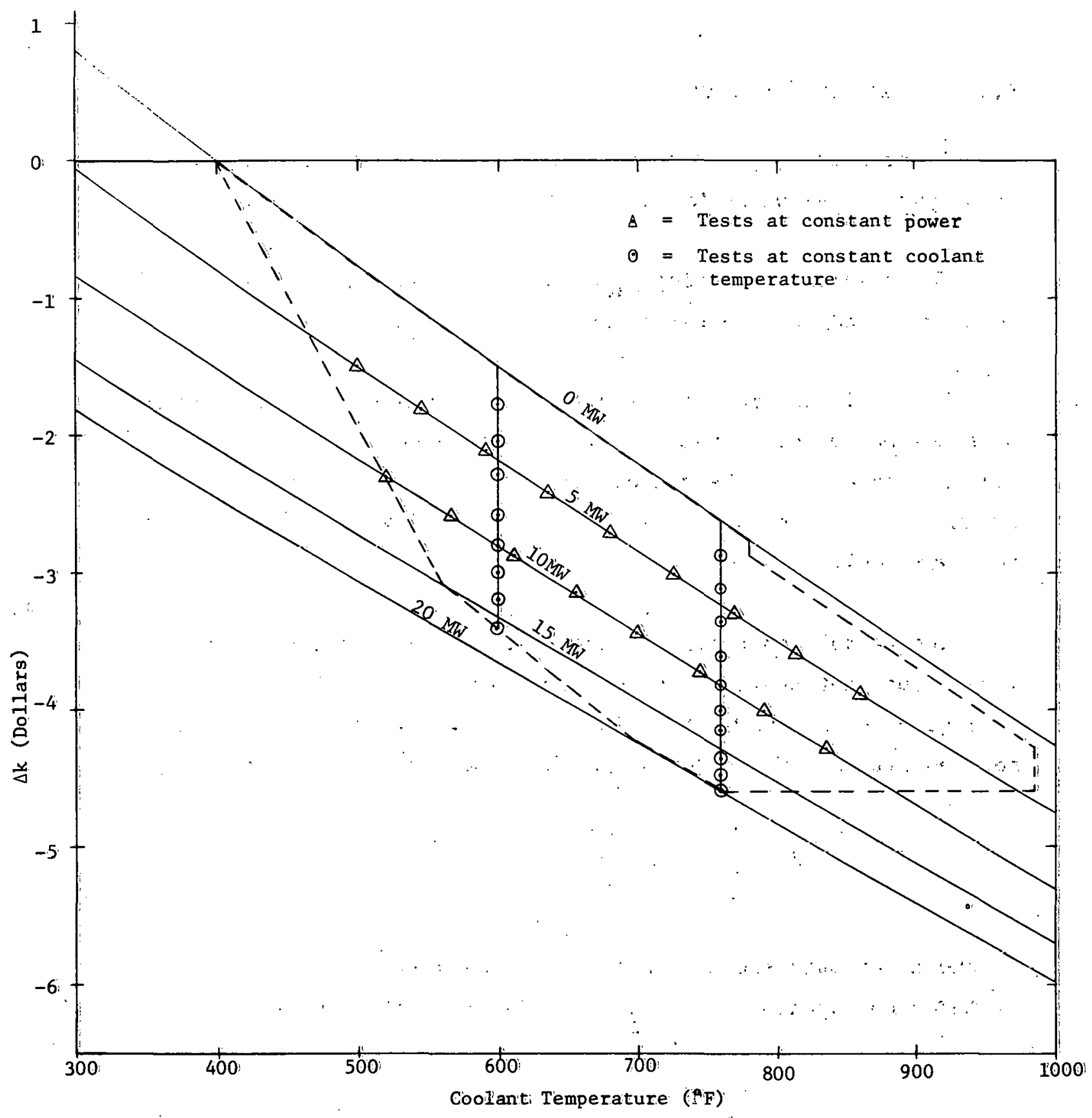

Figure: 4-1. . STATIC TEST POINTS: 


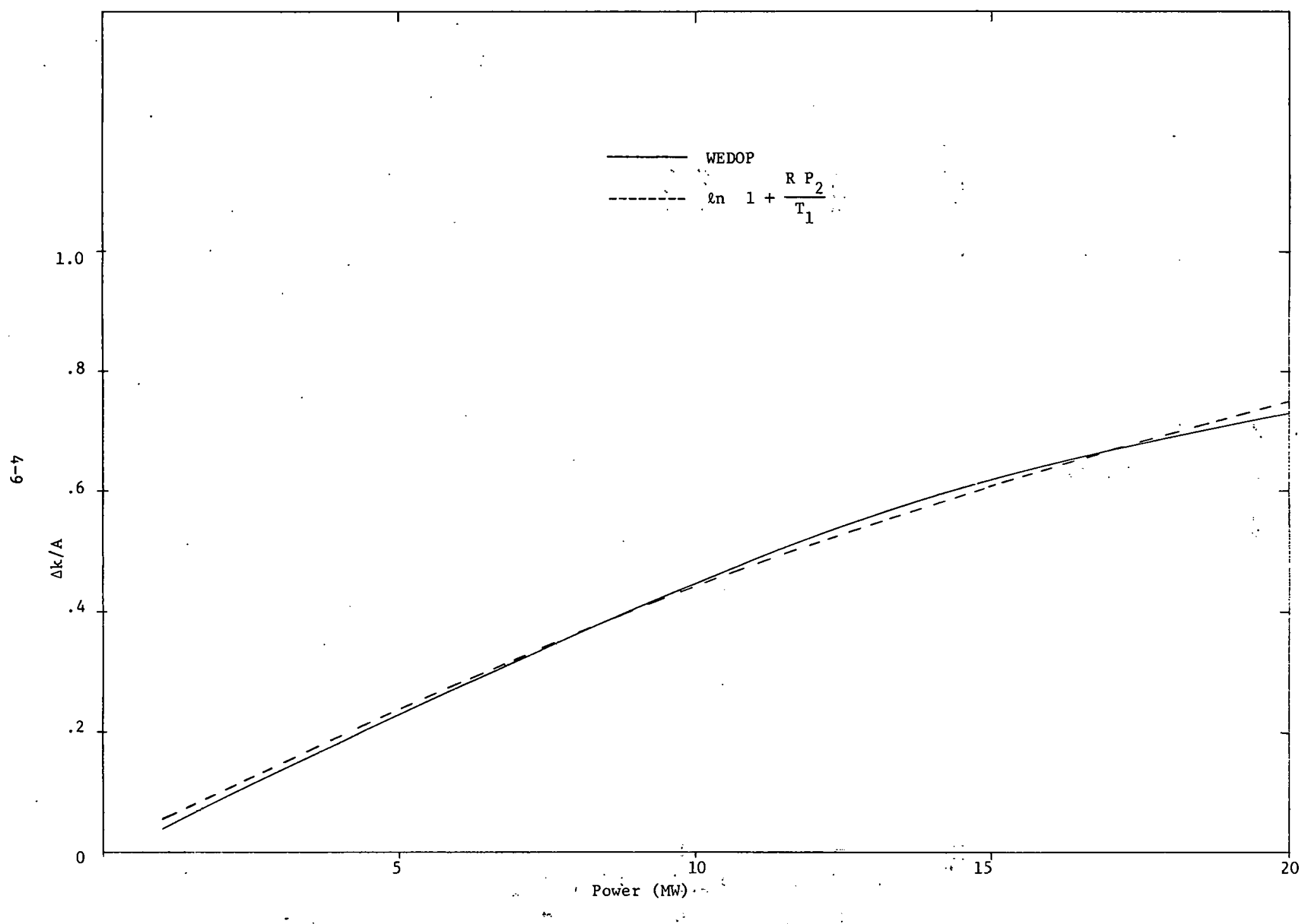

Figure 4-2. DOPPLER REf.CTIVITY VERSUS POWER 
GEAP- -5576

TABLE 4-2.

\section{CALCULATED COEFFICIENTS}

NON-SPATIALLY WEIGHTED MODELS

MODEL

$\ln \left(1+\frac{R_{f} P}{T_{f o}}\right)$

$\ln \left[1+\frac{(a-b P) P}{T_{f o}}\right]$

$\ln \left[1+\frac{a P}{T_{\text {fo }}(1+b P)}\right]$

\section{COEFFICIENT}

$$
R_{f}=65.1
$$

$a=66.44$

$b=0.09$

$a=66.36$
$b=0.0016$ 
Calculations show that use of a model that does not include spatial weighting of the local Doppler effect may underestimate the Doppler effect by approximately ten percent (10\%) relative to the result from the more detailed model used in WEDOP. Thus, if the simple model based on core average fuel temperatures is used in the data analysis, the resulting value of the Doppler constant may be in error about ten percent (10\%). 
GEAP -5576

SECTION $\mathrm{V}$

OSCILLATOR' TESTS

\section{$5.0 \quad$ DISCUSSION}

Iwo kinds of oscillator tests are planned. . These are:

1. Conventional (included zero power) oscillator tests in which a poison rod is oscillated at several frequencies and the reactor power and coolant temperatures are allowed to oscillate about their mean values:

2. Balanced oscillator tests (of which there are two types) in which a poison rod, the primary coolant flow rate, and the secondary coolant flow rate are simultaneously oscillated. The relative phase angle and amplitudes of the reactivity and flow oscillations are adjusted so that one of the following conditions is satisfied:

a. The iulet and outlet corc coolant temperatures remain. constant. This is called a first balanced oscillator test. .

b. The reactor power remains constant. This is called a second balanced oscillator test.

The first and second balanced osci1lator tests are discussed in detail by Caldarola ${ }^{(1)}$ and Russe11.(2)

\subsubsection{ObjucliveE}

Tlie overall objectives of the oscillator tests are:

1. To demonstrate reactor stability to reactivity and to coolant flow (temperature) oscillations at different mean flow rates and at different power levels up to $20 \mathrm{MW}$. 
2. To provide information for the determination of the Doppler and coolant reactivity coefficients at different fuel temperatures.

3. To provide information for the determination of the frequency dependence of the fuel temperature response to a power or coolant temperature oscillation.

The above information will provide an independent check of results obtained from the static tests, and will provide for the determination of the fuel energy coefficient.

\subsubsection{General Procedure}

In order to obtain the above information, the oscillator tests will be performed at a number of different mean power levels, and at a number of different mean primary coolant flow rates at specified mean coolant temperatures. In addition, the oscillator rod and coolant flow rate will be oscillated at a number of different frequencies at each mean power level and mean flow rate. The range of frequency oscillation for the oscillator rod is 20.00125 to $20.5 \mathrm{cps}$, and the range of frequency oscillation for the coolant flow rate is 20.00125 to $0.03 \mathrm{cps}$. The frequency "range of interest is low ( 0.002 to $0.5 \mathrm{cps}$ ) because the fuel time constant for the one-inch diameter SEFOR fuel rod's is $\sim 35 \mathrm{sec}$ ( $?$ ?

All of the oscillations performed at a specified mean power level, flow rate, and coolant temperature will be considered as one oscillator test. 
It is expected that a power oscillation in the range \pm 3 to \$5 will provide a high signal to noise ratio, and that the magnitude of the oscillation will not be so large as to invalidate.tie results of the analysis. If the noise level is very small, it may be possible to lower the amplitude of the power oscillation. Some tests may be performed in which the amplitude of the power oscillation is increased to $\sim \pm 10 \%$ in order to investigate the non-linear characteristics of the system, or, in the first balanced oscillator tests, to increase the ratio of fuel to coolant reactivity effects.

The general procedure for each oscillator test is outlined below:

1. Allow reactor to reach static equilibrium at the specified power, flow, and coolant temperature conditions.

2. Record" background"noise" for each channel while system is at steady-state. The noise can be recorded at several different frequencies by oscillating the poison rod at zero amplitude in the "oscillator test mode" of operation. High frequency noise may also: be determined from data recorded in the "transient mode" of operation and low frequency noise may be determined from data recorded in the "static test mode" of operation. It may be possible to perform this step at another time - during thé static tests for example. It may not be necessary to repeat this step for each different test. 
GEAP-5576

3. Oscillate the reactivity rod and coolant, flow at the desired amplitude and frequency. The variations in the mean power level, and mean coolant flow rate, should be maintained bélow $1 / 2 \%$ and variations in mean system temperatures should be maintained below $20.5^{\circ} \mathrm{F}$ for several successive cycles of oscillation. This may require some initial adjustment of the fine reflector control or of the coolant flow rates.

Recommended frequencies for each test are listed in Section 5.1 through 5.4. It is suggested that the amplitude of the reactivity oscillation be adjusted.so that the expected power oscillation is $\sim \pm 3$ to $\sim \pm 5 \%$. The reactivity-power transfer functions shown in Section 5.1 through 5.4 can be used to estimate the desired amplitude of the reactivity oscillation. The amplitude of the flow oscillation is expected to be $\leq \sim \pm 5 \%$, but will be adjusted to achieve the desired balance (constant power or constant coolant temperature).. The desired amplitudes and frequenries may shange 28 a result of analysis of the data.

4. Allow initial starting transients, including any initial adjustments required, to die out, and record data to determine the amplitude and phase angle of the power, reactivity, flow; and temperature oscillations. The estimated number of cycles for which data.will be recorded are given for each frequency and each test in Section 5.1 through 5.4 .

5. Change to a new frequency of oscillation, while maintaining constant mean test conditions, and repeat steps 3 and 4 until the frequency range of interest has been traversed. 
GEAP -5576

In order to keep the power oscillation in the approximate range of \pm 3 to $\pm 5 \%$, it may be necessary to change the amplitude of the oscillator rod when the frequency of oscillation is changed. In the zero power oscillator tests, for example, (see Figure 5.1.3), a reactivity oscillation of $\pm 5 c$ at $0.5 \mathrm{cps}$ will cause a $\pm 5 \%$, power . oscillation, whereas a reactivity oscillation of on $1 y+0.5 c$ at 0.0016 cps wil1 cause a $\pm 5 \%$ power oscillation.

In order to ensure that all the data from a given oscillator test are properly normalized, whenever the amplitude of the oscillator rod is changed, steps 3 and 4 above should be performed, at the same frequency, using both the new and the old amplitudes. Depending upon the "spread" in the data, it may be necessary to repeat steps 3 and 4 two or three times with each amplitude.

It is suggested that the first frequency of oscillation for each new oscillator test be $0.5 \mathrm{cps}$ and that the frequency spectrum be traversed by successively decreasing the frequency of oscillation.

\subsubsection{Data Collection and Analysis}

When the data acquisition system at the SEFOR site is placed in the oscillator test mode of operation, the power, tlow and temperature signals are sampled every $1 / 32$ of the period of the oscillator rod. The signal which causes the computer to sample the various data channels is provided by the oscillator rod mechanism. Thus, the oscillator mechanism must be used whenever the oscillator test mode of operation is used. The "noise spectrum" and the effects of inde- 
pendent. flow oscillations can be obtained, however, by oscillating the reactivity rod at zero amplitude.

In addition to the data recorded by the data acquisition system, the range in which the flux monitors are operating, the reflector positions, and the amplitude, frequency, and mean position of the oscillating poison rod must be recorded.

It is expected that the analysis of data from a typical oscillator test will include the following steps.

1. Convert the oscillating output signals from the various channels into engineering units. This step can be performed by the data acquisition system at the SEFOR site.

2. Obtain the fundamental frequency components of the oscillating power, flow, reactivity, or temperature signal by evaluating the following integrals:

$$
\begin{aligned}
& R=\frac{2 \omega}{\pi} \quad \sum_{n=1}^{N} q_{n} \cdot \int_{(n-1) \pi / \omega}^{n \pi / \omega} f(t) \sin \omega t d t . N \geq 2 \quad(5.0-1) \\
& I=\frac{2 \omega}{\pi} \quad \sum_{n=11}^{N} q_{n} \int_{(n-1) ; / / \omega}^{n} \pi / \omega
\end{aligned}
$$


The mean value, $M$, of the signal is obtained from the following: integral:

$$
M=\frac{\omega}{N \pi} \int_{0}^{N \pi / \omega} f(t) d t \quad, \quad \begin{aligned}
& N \geq 2 \\
& (N \text { even })
\end{aligned}
$$

If the signal contains drift which can be represented by a polynomial in time, Persson (4) has shown that the contribution of drift to the fundamental components $R$ and $I$ can be eliminated by choosing the weight functions $q_{n}$ to be:

$$
q_{n}=\frac{1}{2^{N-1}} \quad \frac{(N-1) !}{(N-n) !(n-1) !}
$$

If the signal $f(t)$ contains no drift, either the above choice of $q_{n}$ or the conventional chnice

$$
q_{n}=\frac{1}{N} \quad(N \text { even })
$$

will yield the same values (within the uncertainties due to noise) for the coefficients $R$ and $I$.

A computer program has been specified which will allow either choice for the weight functions $q_{n}$. A comparison of the use of the weight functions and the effect of discrete sampling is described in Appendix IV. 
If desired, the higher harmonics in the test data can also be determined. The $m$-th coefficient $R_{m}$ is determined by replacing sin $\omega t$ in $(5.0-1)$ by sin $m \omega t$ and the $m$-th coefficient. I by replacing cos $\omega t$ in $(5.0-2)$ by $\cos m \omega t$. Either weight function (5.0-4) or (5.0-5) may be used if $M$ is odd, but "only the conventional weight function: $q_{n}=\frac{1}{N}$ should be used if $m$ is even, since for $m$ even the weight functions given by (5.0-4) will give erronous resulțs:

3. The. "noise" frequency: spectrum is determined by performing the above analysis, at several frequencies $\omega$, on the output signals when neither the reactivity nor the flow is being oscillated. It is assumed that the "noise" frequency spectrum does not change during the test, and that a "noise". correction can be. applied to the values of $R$ and $I$ that are determined from the oscillator test data.

4. The standard error in the fundamental components $R$. and $I$ can be estimated by performing the same test a number of times. The "spread" in the data can be estimated by determining $R$ and I from a number of different cycles of data that are obtained during the same test.

5. Calculate the transfer function between two desired signals $f(t)$ and $g(t)$ by using the coefficients obtain in step 2; Once the quantities $R_{f}$ and $I_{f}$ have been determined for channel $f$ and the corresponding quantities determined for channel $\mathrm{g}$, the complex quantities $f$ and $g$ can be defined by:

$$
\begin{aligned}
& f=R_{f}+i I_{f} \\
& g=R_{g}+i I_{g}
\end{aligned}
$$


where $i \equiv \sqrt{-1}$. The complex transfer function $T(i \omega)=R_{T}+i I_{T}$ is then determined from

$$
\mathrm{T}=\frac{\mathrm{f}}{\mathrm{g}}
$$

Based on the assumption that the standard errors $\sigma_{f}$ in $R_{f}$ and $\delta_{f}$ in $I_{f}$ are random and uncorrelated, and that the standard errors in $g$ are likewise uncorrelated, the standard error $\sigma_{T}$ in $R_{T}$ and $\delta_{T}$ in $I_{T}$ is calculated from the following expression."

$$
\begin{aligned}
& \sigma_{\mathrm{T}}^{2}=\mathrm{R}^{2}\left(\frac{\mathrm{T}}{\mathrm{f}}\right) \sigma_{\mathrm{f}}^{2}+\mathrm{I}^{2}\left(\frac{\mathrm{T}}{\mathrm{f}}\right) \delta_{\mathrm{f}}^{2}+\mathrm{R}^{2}(\mathrm{~T} / \mathrm{g}) \sigma_{\mathrm{g}}^{2}+\mathrm{I}^{2}(\mathrm{~T} / \mathrm{g}) \delta_{\mathrm{g}}^{2}(5.0-7) \\
& \delta_{\mathrm{T}}^{2}=\mathrm{I}^{2}\left(\frac{\mathrm{T}}{\mathrm{f}}\right) \sigma_{\mathrm{f}}^{2}+\mathrm{R}^{2}\left(\frac{\mathrm{T}}{\mathrm{f}}\right) \delta_{\mathrm{f}}^{2}+\mathrm{I}^{2}(\mathrm{~T} / \mathrm{g}) \sigma_{\mathrm{g}}^{2}+\mathrm{R}^{2}(\mathrm{~T} / \mathrm{g}) \delta_{\mathrm{g}}^{2}(5.0-8)
\end{aligned}
$$

where $R(x)$ denotes the real part of $X$ and $I(x)$ denotes the imaginary part of $x$.

The amplitude $A_{f}$ and phase angle $\phi_{f}$ of the complex function $f$ are calculated from the following relations:

$$
\begin{aligned}
& A_{f}=\sqrt{R_{f}^{2}+I_{f}^{2}} \\
& \phi_{f}=\tan ^{-1} I_{f / R_{f}}
\end{aligned}
$$

The standard errors $\nu_{f}$ in $A_{f}$ and $\mu_{f}$ in $\phi_{f}$ are calculated from:

$$
\begin{aligned}
& \nu_{\mathrm{f}}^{2}=\frac{I_{\mathrm{A}}^{2}}{\mathrm{f}}\left[\mathrm{R}_{\mathrm{f}}^{2} \sigma_{\mathrm{f}}^{2}+\mathrm{I}_{\mathrm{f}}^{2} \delta_{\mathrm{f}}^{2}\right] \\
& u_{\mathrm{f}}^{2}=\frac{1}{\mathrm{~A}_{\mathrm{f}}^{\prime}}\left[I_{\mathrm{f}}^{2} \sigma_{\mathrm{f}}^{2}+\mathrm{R}_{\mathrm{f}}^{2} \delta_{\mathrm{f}}^{2}\right]
\end{aligned}
$$


The amplitude $A_{T}$, and phase angle $\phi_{T}$ of the transfer function $T$ are gịven by:

$$
\begin{aligned}
& A_{T}=A_{f} / A_{g} \\
& \phi_{T}=\phi_{f}-\phi_{g}
\end{aligned}
$$

and the uncertainties $\nu_{T}$ and $\mu_{T}$ are calculated from the following expressions:

$$
\begin{aligned}
& \frac{\nu_{T}^{2}}{A_{T}^{2}}=\left(\frac{\nu_{f}}{A_{f}}\right)^{2}+\left(\frac{\nu_{g}}{A_{g}}\right)^{2} \\
& \mu_{T}^{2}=\mu_{f}^{2}+\mu_{g}^{2}
\end{aligned}
$$

6. In the conventional and first balanced oscillator tests, the transform, $h(i w)$, of the feedback kernel is calculated from the inverse, $z_{0}$; of the zero power-reactivity transfer function, $G_{0}$, and from the inverse,; $z^{\prime}$, of: the at: power-reactivity transfer. function, $G$, by means of the following expression:

$$
h(i \omega)=\frac{1}{P}\left(z_{0}-z\right)=\frac{1}{P}\left(\frac{1}{G}-\frac{1}{G}\right)
$$

where $P^{2}$ is the power. level at which $z$ is determined.

The transfer function $z$ can be written as:

$$
Z=\frac{\Delta K M}{\Delta P}=M T: \equiv M\left(\frac{R_{f}+i I_{f}}{R_{g}+i I_{g}}\right)
$$

where $M$ is: the mean: value of the power signal, and $T$ is defined by equation $(5.0-6)$ when $f=. \Delta k$, and $g: \Delta P$. 
The standard errors $\sigma_{z}$ in $R_{z}$ and $\delta_{z}$ in $I_{z}$ are thus calculated from:

$$
\begin{aligned}
& \sigma_{z}^{2}=R_{z}^{2}\left(\frac{\sigma_{M}}{M}\right)^{2}+M^{2} \sigma_{T}^{2} \\
& \delta_{z}^{2}=I_{z}^{2}\left(\frac{\sigma_{M}}{M}\right)^{2}+M^{2} \sigma_{T}^{2}
\end{aligned}
$$

where $\sigma_{\mathrm{T}}^{2}$ and $\delta_{\mathrm{T}}^{2}$ are given by equations (5.0-7) and (5.0-8). The quantity $\sigma_{M}^{2}$ is the random error in the mean signal from the flux detectors and does not include bias errors due to the uncertainty. in absolute power level.

The standard deviations in $R_{h}$ and $I_{h}$ are calculated from:

$$
\begin{aligned}
& \sigma_{h}^{2}=\frac{1}{p^{2}}\left[\sigma_{z 0}^{2}+\sigma_{z}^{2}\right]+R_{h}^{2}\left(\sigma_{p} / p\right)^{2} \\
& \delta_{h}^{2}=\frac{1}{p^{2}}\left[\delta_{z o}^{2}+\delta_{z}^{2}\right]+I_{h}^{2}\left(\sigma_{p} / p\right)^{2}
\end{aligned}
$$

where $\sigma_{p}$ is the random error in the mean power level $P$.

7. In the conventional, or the first balanced, oscillator tests, the product $\mathrm{Ph} / \mathrm{z}_{\mathrm{o}}=\mathrm{PhG}_{\mathrm{o}}$ can be plotted to obtain information concerning redator stability. The reactor would be lincar unetable (if $h$ did not depend upon the power) at a power level where $\mathrm{PhG}_{0}=1.0$. Regardless of whether or not $P h G_{0}=1.0$, the phase margin, and gain margin (if defined) can be determined from the conventional, or first balanced, oscillator tests. Reactor stability, gain and phase margins are discussed in several references $(5,6,7)$ and the discussions need not be repeated here. 
8. " Fit $h(i \omega)$, or transfer functions such as the one between coolant temperature and input reactivity, to a model in order to obtain reactivity coefficients and frequency response functions. The models for the various frequency response functions and transfer functions are described in Section 2.5.

9. In addition to the above, it may be desirable to check for "consïstency" between the real' and imaginary parts and/or the amplitudé and phase of transfer functions calculated in step 5 above, or to check for "consistency" in the data" obtained in step 2 .

It can be shown (8) that if the Fourier transform, $F(\omega)$, of a causal function is analytic and bounded in the lower half $\omega$ plane, including the real axis, the real part, $R(\omega)$, and imaginary part, $I(\omega)$, of $F(\omega)$ are related, to within an arbitrary additive constant, by the following expressions:

$$
\begin{array}{r}
R(\omega)=\frac{-1}{\pi} \quad P \int_{-\infty}^{\infty} \frac{I(u)}{u-\omega} d u \\
\cdots \\
I(\omega)=\frac{1}{\pi} \quad P \cdot \int_{-\infty}^{\infty} \frac{R(u)}{u-\dot{\omega}} d u
\end{array}
$$

where $\mathrm{P}$ denotes the principal value.

If the system: is minimum phase, (i.e., if the logarithm of... the amplitude $A(\omega)$, and the phase angle. $\phi(\omega)$ are analytic in the lower .half w plane including the real axis) the following relations. are valid:

$$
\ln A(\omega)=\frac{-1}{\pi} P \int_{-\infty}^{\infty} \frac{\phi(u)}{u-\omega} d u
$$


and

$$
\begin{aligned}
\phi(\omega) & =\frac{1}{\pi} P \int_{-\infty}^{\infty} \frac{\ln A(u)}{u-\omega} d u \\
\therefore \quad & =\frac{1}{\pi} P \int_{-\infty}^{\infty} \frac{d\left[\ln \Lambda\left(\omega \mathrm{e}^{\lambda}\right)\right]}{\mathrm{d} \lambda} \ln \operatorname{coth}\left|\frac{\lambda}{2}\right| \mathrm{d} \lambda
\end{aligned}
$$

Thus, for example, the experimental values of $I(\omega)$ : can be used in equation (5.0-10) to calculate vales of $R(\omega)$ which can then be compared with the measured values of $R$. The calculated and measured values of $R(\omega)$ should agree, within the limits of the experimental measurements and accuracy of the numerical integration.

In addition, the consistency of the amplitude and phase data. can be checked in order to determine whether or not the system is minimum phase. The real and imaginary components should always be "consistent". The amplitude and phase data will not be consistent, however, if the system is non-minimum phase, e.g. if the transfer function in question contains a pure delay of the form $e^{-i \omega t}$.

\subsection{ZERO POWER USCILLATOR TEST}

A zero power oscillator test is a conventional oscillator. test. which is performed at a power level which is sufficiently low that changes in power level on the order of $10 \%$ cause an indiscernible change in fuel or coolant temperature.

For the zero power oscillator tests, a poison rod is successively oscillated. (sinusoidally) at several.different frequencies. The reactor 
power is allowed to oscillate with amplitudes up to $10 \%$ of the mean power level. The mean power level and the coolant temperature are held constant during the test.

\section{1 .1 Objective}

The primary objective of the zero power oscillator test is to determine the power-reactivity transfer function for the critical reactor at a power level which is sufficiently low that reactivity feedback effects can be neglected. The reactor should be critical, and feedback effects will be small for power: levels of $\sim 10 \mathrm{~kW}$. The absolute coolant temperature is unimportant as long as it is held constant during the test. The effects of coolant temperature drift are discussed in Section 5.1.2.

A possible secondary objective (the determination of the fraction of fissions caused by $\mathrm{U}-238$ ) is discussed in Section 5.1 .3 .

\subsubsection{General: Procedure}

In general, the procedure for the zero power oscillator test is the same as for all the oscillator tests and is described in Section 5.0.2. Only the oscillating power and reactivity (oscillator rod position) signals are of primary interest, however, and other signals, such as coolant and fuel temperatures or primary and secondary coolant flow rates, need be observed only to the extent necessary to ensure that they remain constant during the test.

The frequencies of oscillation for the zero power tests are the same as for the conventional oscillator test and it is suggested that 5 frequencies per decade be used over the frequency range of interest ( 20.002 to $0.5 \mathrm{cps}$ ). A possible sequence of frequencies and the number of cycles of data (after the initial transients have died out) are given in Table 5-2. 
Determination of the zero power transfer function at lower frequencies may be difficult due to the reactivity effect that results from a small slow drift in the core temperature. At zero power, the change in reactivity as a result of a core temperature change is $20.6 \mathrm{c} /{ }^{\circ} \mathrm{F}$. For a linear drift of $" \mathrm{~A} "{ }^{\circ} \mathrm{F} / \mathrm{sec}$, the power increases (if initially critical) approximately as $\exp \left(0.3 \mathrm{At}^{2} / \mathrm{T}\right)$ where $\mathrm{T} \sim 1000$ and $t$ is the time in sec. Thus, a drift of only $1^{\circ} \mathrm{F}$ per 10 minutes will cause an increase in the mean power level of $238 \%$ during one of oscillation at a frequency of 0.00125 cps.

\subsubsection{Data Collection and Analysis}

The data will be collected and analyzed as described in steps 1 through 5 of Section 5.0.3 to determine the zero power. transfer function $G_{0}=\Delta P / P_{0} / \Delta K$, and to estimate the standard errors in the real and imaginary parts of $G_{0}$.

The calculated value of the zero power transfer function at 0.5 cps can be used to obtain a "dynamic" measurement of the oscillator rod worth as follows. Since the calculated amplitude of the zero power transfer function at $0.5 \mathrm{cps}$ is 1.055 , the rod worth, in cents, is 1.055 times the percent power oscillation at 0.5 cps. The "dynamic" measurement of the worth can be compared with the "static" worth that is measured when the oscillator rod is moved the same distance in a static test.

In addition to the above, it may be possible to determine the delayed neutron fraction $\beta$ and the fraction of fission neutrons, $\mathrm{q}$, which are produced by $\mathrm{U}-238$. The procedure for this analysis is described below. 
- The inverse, $z_{\text {ar }}$, of the zero power transfer function may bes written as follows:

$$
z_{0}=\frac{1}{G_{0}}=i \omega \frac{\ell}{\beta}+\frac{1}{\beta} \sum_{m=1}^{M} \beta_{m} q_{m} \sum_{n=1}^{N} a_{m n} e_{m n} /\left(i \omega+\lambda_{m n}\right)
$$

where $i=\sqrt{-1}$; $w$ is the frequency of oscillation; $M$ is the number of fissionable isotopes; $N$ is the number of delayed neutron groups; $\beta_{m}$ is the delayed neutron fraction of isotope $M$; $\ell / B$ is the ratio of the prompt neutron lifetime to the delayed frction; ${ }_{m n}, e_{m n}$, and $\lambda_{\mathrm{mn}}$ are the relative abundance, the effectiveness (relative to prompt neutrons), and the decay constants, respectively, for the delayed neutrons in group $n$ from isotope $m ; q_{m}$ is the fraction of the fission neutrons. which are produced by isotope $\mathrm{m}$; and the delayed neutron fraction, $\beta$, is defined by:

$$
\beta=\sum_{m=1}^{M} \beta_{m} q_{m} \sum_{n=1}^{N} a_{m n} e_{m n}
$$

The delayed neutron parameters, $\beta_{\mathrm{m}}, \mathrm{a}_{\mathrm{mn}}$, and $\lambda$ mn are assumed to be known, and equal to; the values: given by: Keepin." The fractions of fission neutrons produced have: been calculated for SEFOR by Reynolds and Stewart: (10), The calculated values of the $e_{m n}$ for SEFOR are constant, tos within $11 \%$, and equal to 0.91 . (11) The values of $\ell / \beta$ in SEFOR wiIl be. determined by noise analysis (see section 3.11). The calculated value. of $B$ is 0.0032 (10) 
In SEFOR, $297.5 \%$ of the fissions occur in the U-238 and the $\mathrm{Pu}-239 .(10)$ Thus, the inverse of the zero power transfer function may be approximated by the following expression:

$$
z_{0}=i \omega\left[\frac{\ell}{\beta}+\frac{\varepsilon \beta_{1}}{\beta} \text { (1-f) } \sum_{n=1}^{N} \frac{a_{n 1}}{i \omega+\lambda_{n 1}}+\frac{\varepsilon \beta_{2} f}{\beta} \sum_{n=1}^{N} \frac{a_{n 2}}{i \omega+\lambda_{n 2}}\right]
$$

where the subscripts 1 and 2 refer to the delayed neutron parameters for $\mathrm{Pu}-239$ and for $\mathrm{U}-238$, respectively, where $\dot{\varepsilon}$ is the effectiveness $(0.91)$, and where $f$ is the fraction of 'fission neutrons produced by ' $\mathrm{U}-238$. The approximate expression above over predicts the calculated fraction of fission neutrons produced by $U-238$ by $\sim 0.4 \%$ and over predicts the fraction of fission neutrons produced by $\mathrm{Pu}-239$; (1-f); by น2. $1 \%$.

The simplified model for $z_{0}$ can be used, along with the curve fitting procedure described in Appendix $V$, to determine the value of $f$ for which the sum of the weighted squares of the residuals for the real and imaginary parts of: $z_{0}$ are a minimum.

For this case, the least squares procedure outlined in Appendix $V$ is particularly simple and the resultant equations are given below:

Let $\omega_{m}(m=1,2, \ldots M)$ be the frequencies at which the real part. $R_{m}$, and the imaginary part, $I_{m}$, of the inverse, $z_{o}(i \omega)$, of the zero power transfer function are measured. Let $\sigma_{\mathrm{Rm}}$ and $\sigma_{\mathrm{Im}}$ be the estimated standard errors in $R_{m}$ and $I_{m}$, respectively. Let $\sigma_{0}$ be an arbitrary constant and define:

$$
\therefore \quad g_{j}=\frac{\varepsilon \beta_{j}}{\beta} \quad(j=1,2)
$$




$$
\begin{array}{lll}
R_{m j}=\omega_{m}^{2} & \sum_{n=1}^{N}\left[\frac{a_{n j}}{\lambda_{n j}^{2}+\omega_{m}^{2}}\right] & (j=1,2) \\
I_{m j}=\omega_{m} & \sum_{n=1}^{N}\left[\frac{a_{n j} \lambda_{n j}}{\lambda_{n j}^{2}+\omega_{m}^{2}}\right] & (j=1,2)
\end{array}
$$

where $j=1$ refers to the $\mathrm{Pu}-239$ parameters and $\mathrm{j}=2$ refers to the $\mathrm{U}-238$ parameters, and where the estimated value of $\beta$ is used in the calculation of $g_{1}$ and $g_{2}$.

The sum of the weighted squares of the residuals in the real and imaginary parts of $z_{0}$ can be written as, follows:

$$
\begin{aligned}
S & =\sum_{m=1}^{M} \frac{\sigma_{0}^{2}}{\sigma_{R m}^{2}}\left[R_{m}-g_{1} R_{m 1}-f\left(g_{2} R_{m 2}-g_{1} R_{m 1}\right)\right]^{2} \\
& +\sum_{m=1}^{M} \frac{\sigma^{2}}{\sigma^{2}}\left[I_{m}-\omega_{m} \frac{l}{B}-g_{1} I_{m 1}-f\left(g_{2} I_{m 2}-g_{1} I_{m 1}\right)\right]^{2}
\end{aligned}
$$

Minimizing $S$ with respect to $f$, one obtains the following equation:

$\mathbf{f}=\frac{1}{Q} \sum_{m=1}^{M}\left\{\frac{\sigma_{0}^{2}}{\frac{o}{\sigma_{R m}^{2}}}\left[R_{m}-g_{1} R_{m 1}\right]\left[g_{2} R_{m 2}-g_{1} R_{m 1}\right]+\frac{\sigma_{0}^{2}}{\sigma_{I m}^{2}}\left[I_{m}-\omega_{m} \frac{\ell}{\beta}-g_{1} I_{m l}\right]\left[g_{2} I_{m 2}-g_{1} I_{m l}\right]\right\}$

where:

$$
Q=\sum_{m=1}^{M}\left\{\frac{\sigma_{0}^{2}}{\sigma_{\mathrm{Rm}}^{2}}\left[g_{2} R_{m 2}-g_{1} R_{m 1}\right]^{2}+\frac{\sigma_{o}^{2}}{\sigma_{I m}^{2}}\left[g_{2} I_{m 2}-g_{1} I_{m l}\right]^{2}\right\} .
$$


The estimated standard error, $\sigma_{f}$, in $f$ is then obtained from the following equation:

$$
\sigma_{\mathrm{f}}^{2}=\frac{1}{\mathrm{Q}} \frac{\mathrm{S}}{2 \mathrm{M}-1}
$$

where the value of $f$ obtained from (5.1-3) is used in (5.1-2) to calculate S.

The value of $f$ obtained above can then be used to calculate $B$ from the following expression:

$$
B=\varepsilon\left[\beta_{1}(1-f)+\beta_{2} f\right]
$$

If the value of $\beta$ obtained from this expression does not agree with the value that was used in the initial calculation of $g_{1}$ and $g_{2}$, the new value of $\beta$ can be used to recalculate the functions $g_{1}$ and $g_{2}$ and the above procedure for the determination of $f$ can be repeated.

\subsubsection{Expected Results}

The amplitude and phase of the zero power transfer function, $G_{0}$, is shown in Figure 5.1-1 and 5.1-2 for different fractions of fissions in $U-238$. The calculated effective $f$ for the simplified model, equation $(5.1-1)$ is 0.108 , and the calculated value of $\beta$ is 0.0031 .

The expected amplitude and phase of the zero power transfer function is shown in Figure 5.1-3 and 5.1-4, for cases in which the reactor is subcritical. 


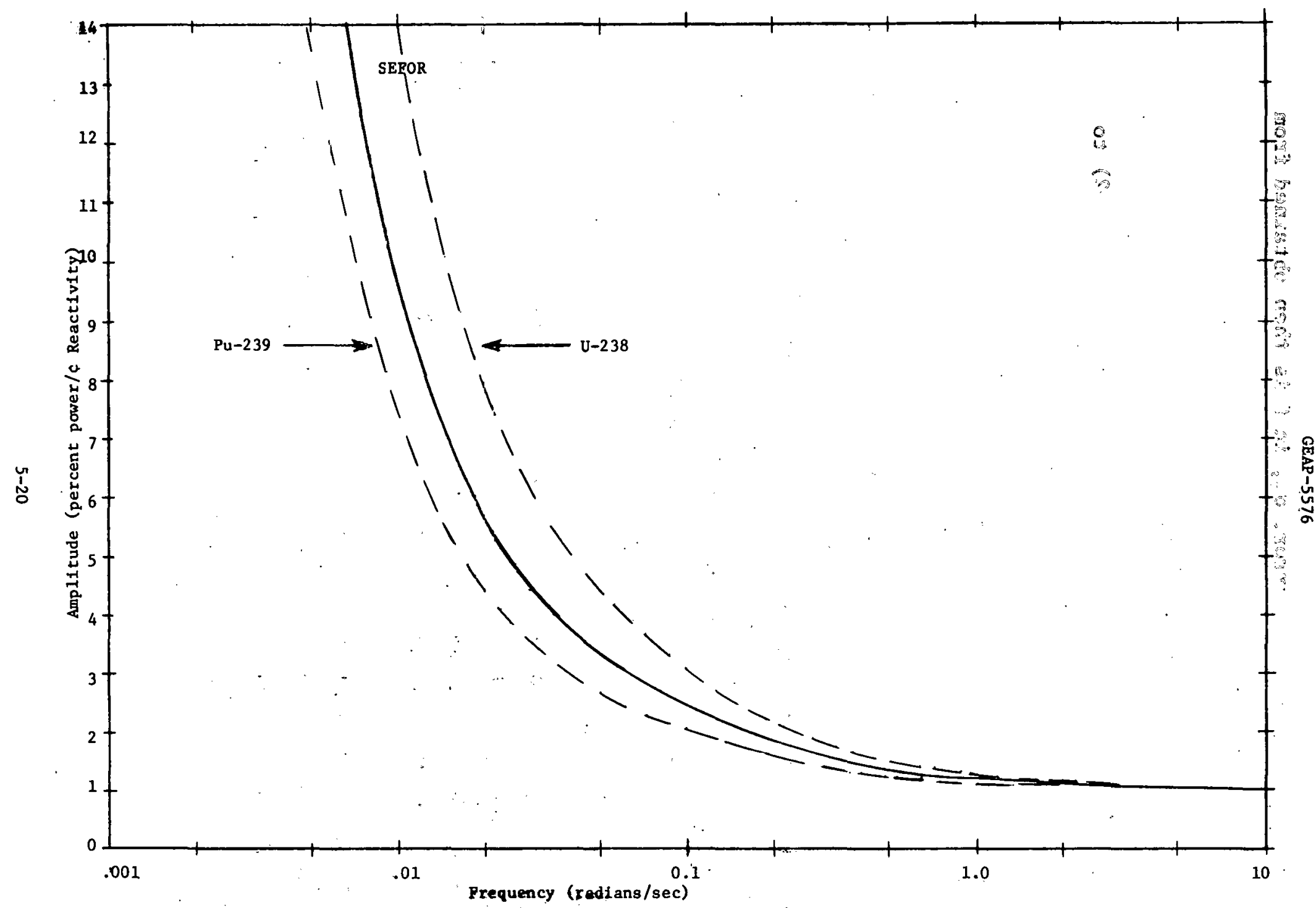

Figure 5.1-1. AMPLITUDE FOR PURE PU-239, SEFOR, AND PURE U-238 ZERO POWER TRANSFER-FUNCTION 


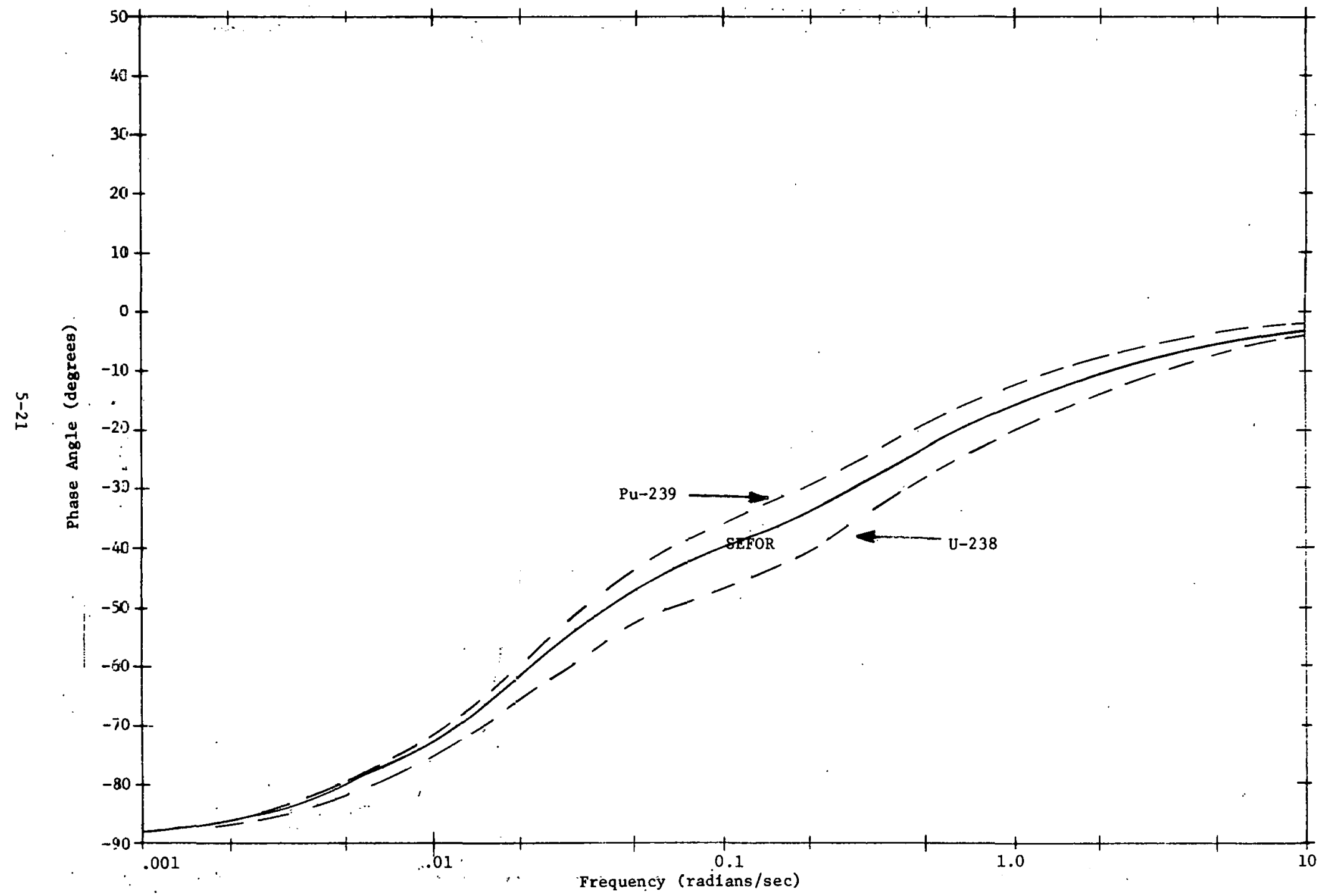

F1gure 5.1-2. PLASE ANGLE FOR PUEE PU-239, SEFOR, AND U-238 ZERD POWER TEANSFER FUNCTIONS 


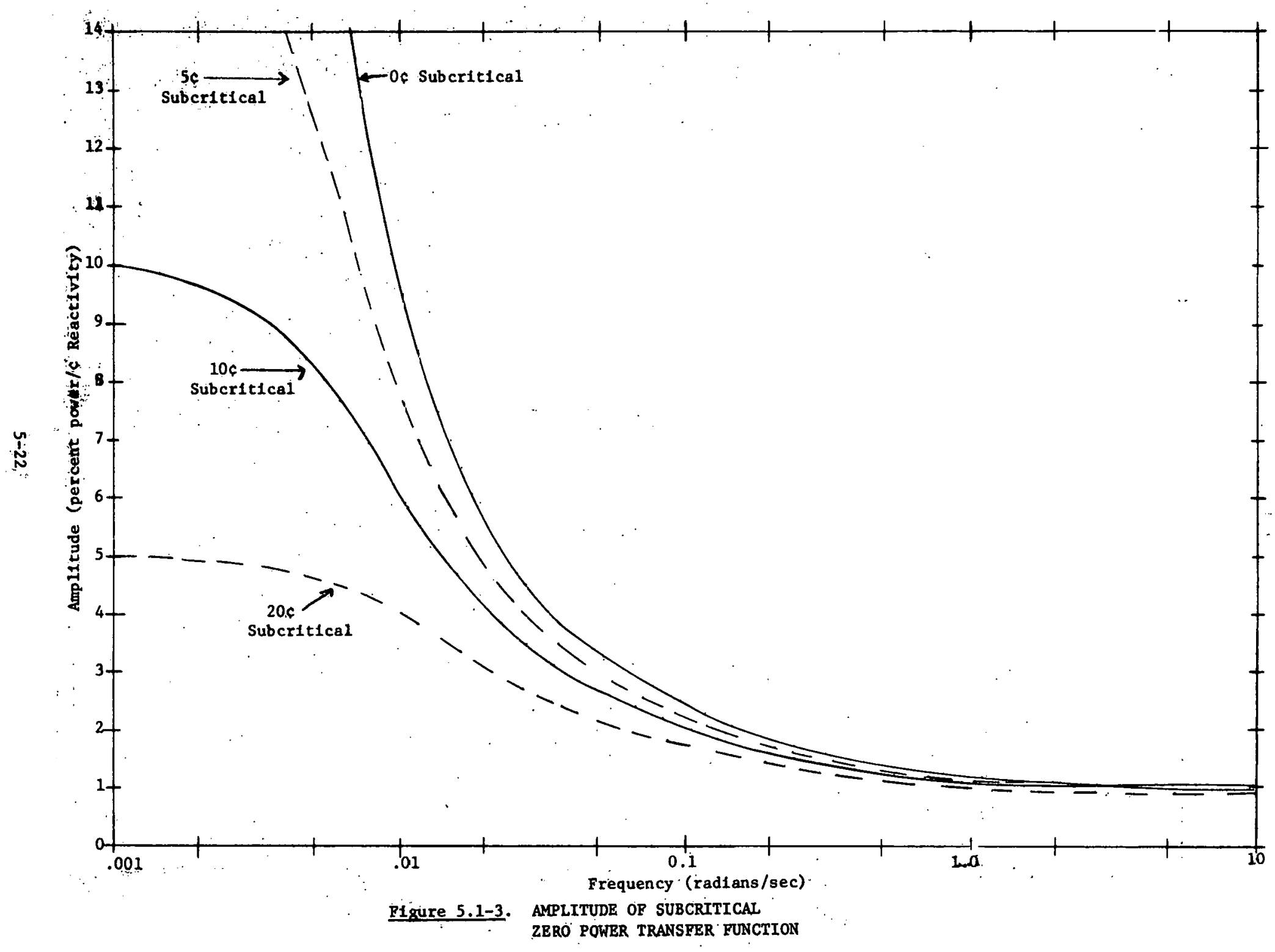




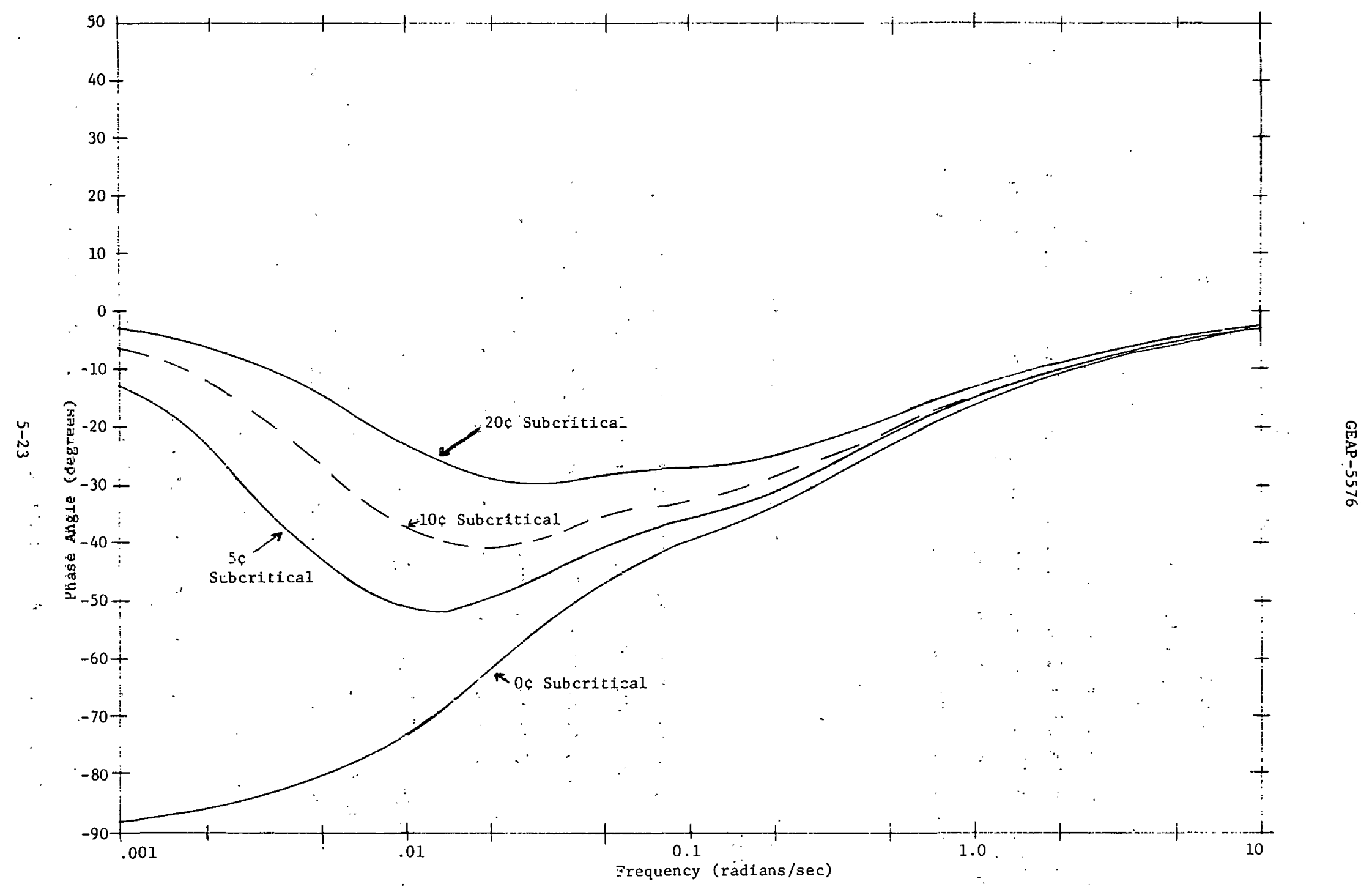

Figure 5.1-4. PHASE ANGLE OF SUBCRITICAL

ZERO POWER TRANSFER FUNCTION 


\subsection{CONVENTIONAL OSCILLATOR TESTS}

In the conventional oscillator tests, a poison rod is successively oscillated (sinusoidally) at several different frequencies. The reactor power and system temperatures are allowed to oscillate with anplicudes up to approximately $10 \%$ of their mean values. Although the mean power level and mean coolant temperature should not deviate (within $20.5 \%$ or $0.5^{\circ} \mathrm{F}$ ) from their mean values during the test, no attempt is made to dampen either the power or the coolant temperature oscillation by means of a coolant flow oscillation; i.e. the ccolant flow rate is held constant.

\subsubsection{Objective}

The objectives of the conventional oscillator tests and their analysis are:

1. to demonstrate reactor stability at different flow rates and at different power levels.up to $20 \mathrm{MW}$.

2. to determine the Doppler and coolant reactivity coefficient.

3. to determine the frequency dependence of the fuel temperature response to a power oscillation.

\subsubsection{General Procedure and Test Conditions}

In general, the procedure for the conventional oscillator test is the same as for all the oscillator tests and is outlined in Section 5.0.3.

The signals of primary interest are:

1. The reactor power (neutron flux)

2. The oscillator rod (reactivity) signal

3. The inlet and outlet, coolant.temperature signals from the instrumented fuel assemblies.:. 
4. The fuel temperature signals from the instrumented fuel assemblies.

In addition, the flow rate, absolute mean power level, system temperatures,' and reflector positions should be recorded.

The test conditions for the conventional oscillator tests are given in Table $5-1$.

The number of frequencies, the sequence of frequencies, and the number of cycles of data to be collected at each frequency depend to a great extent upon the observed results; e.g. the appearance of an unexpected "resonance" may influence the number of frequencies; or the amount of drift or "noise" may influence the number of cycles of collected data.

Although, as pointed out above, it may prove to be inapplicable, a possible sequence of frequencies for each conventional oscillator test, along with the tentative number of cycles of data (after the initial transients have died out) for each frequency are shown in Table 5-2. With this sequence, the test could be performed using the frequencies indicated by Case A ( 5 frequencies per decade) and then repeated (as a check) using the frequencies indicated by Case $B$ ( $2-1 / 2$ frequencies per decade). Any additional checks could also be performed using the frequencies incicated by Case $B$. 


$$
\begin{gathered}
\text { GEAP }-5576 \\
\therefore \\
\text { TABLE } 5-1
\end{gathered}
$$

CONVENTIONAL OSCILLATOR TESTS

Average Coolant Temperature $=760^{\circ} \mathrm{F}$

Power

(MW)

1

5

10

15

19
Flòw

(\%)

50

100

50

30

100.

50

75

95
Core $\Delta \mathrm{T}$

$\left({ }^{\circ} \mathrm{F}\right)$

12

30

60

100

60

120

120

120 
GEAP-5576

TABLE 5-2.

POSSIBLE FREQUENCIES FOR CONVENTIONAL OSEILLATOR TESTS

Frequencies
(cycles/sec)

0.50

0.25

0.16

0.10

0.063

0.040

0.025

0.016

0.010

0.0063

0.0040

0.0025

0.0016
Number

of cycles

15

15

15

15

15

15

15

10

10

7

5

3

3

Minutes for Specified Cycles

\section{Case A}

i

1

2

3

4

6

10

11

17

19

2.1

20

32

\section{Case B}

1

3

10

17

21

32

\section{2 .3 Data Collection and Analysis}

\subsubsection{Discussion}

The general procedure for data collection and analysis are described in Section 5.0.3 and these procedures can be used, where applicable, in the conventional oscillator tests.

As in the zero power oscillator tests (see Section 5.0.3), the "dynamic" measurement of the oscillator rod worth can be compared with the "static" measurement of the rod worth. The calculated amplitude of 
the power transfer function at $20 \mathrm{MW}$ is 1.048, if the fraction: of heat which is generated in the coolant is 0.035 or 1.056 ; if no heat is generated in the coolant. The difference between the amplitude of the at-power-transfer-function and the amplitude of the zero-power-transfer-function (1.055) is approximately linear with power between 0 and $20 \mathrm{MW}$ at a frequency of $0.5 \mathrm{cps}$.

It is planned that the reactivity coefficients and parameters in the fuel frequency response functions w1ll be determined for each of the oscillator tests. It is expected that the Doppler reactivity coefficients and the frequency response parameters will change as the mean power or coolant temperature is changed.

The data from each oscillator test will, be treated as described in Section 5.0.3, in order to provide "measured" transfer functions and estimated standard errors in the transfer functions at several different frequencies. The transfer functions, and the estimated standard errors, will then be used to obtain the reactivity coefficlents and fuel frequency response parameters by means of a least square fitting of the models to the "measured" data.

A derivation of general least squares fitting equations based on the derivation described by Deming ${ }^{(12)}$ is shown in Appendix $v$. The fitting equation for the conventional oscillator tests is described in more detail below.

The mathematical model for the conventional oscillator tests is given in Section 2.5. In terms of this mode1, the "condition equations" can be written as follows:

$$
L(i \omega)=h(i \omega)-\alpha_{f} F(i \omega) X_{f}(i \omega)-\left[C_{f} F_{s}(i \omega)+c_{q}\right] U(i \omega)-c_{1} U_{1}(i \omega)=0
$$


This expression represents two equations since it is desired that . both the real and imaginary part of $\mathrm{L}$ be equal to zero.

Thus, the condition equations can be written as: $\theta \equiv \operatorname{Real}(L)=0$ and $\phi=$ Imaginary $(L)=0$.

The "measured" quantities are $h(i \omega), U(i \omega)$, and $U_{i}(i \omega)$. The term $x_{f}(i \omega)$ is a known calculated quantity (see.Appendix II), and $F_{\text {av }}(i \omega)$ and $F_{S}(i \omega)$ are expressed in terms of two parameters $\gamma$ and $\checkmark$ as shown in Section 2.5 .

In the following discussion, it will be assumed that there are six unknown parameters to be determined. These parameters are $\alpha_{f}$, $\gamma, \nu, C_{f}, C_{q}$, and $C_{i}$. In case it is desired to treat any of these parameters as a known quantity, the equations shown below may be modified in a manner to be described at the end of this section.

In the fitting of the model to the conventional oscillator test data, a total of $6 \mathrm{xN}$ (where $\mathrm{N}$ is the total number of frequencies of oscillation) data points are used. These are the real and imaginary components of $h$, of $U$, and of $U_{i}$ at each of the frequencies of oscillation. Let these data points be stored in a matrix, $x$, and let the elements $x(m, n)$ of the $x$ matrix be defined so that at each frequency, $\omega_{n}$, the measured values can be written as:

$$
\begin{aligned}
h\left(i \omega_{n}\right) & =x(1, n)+i x(2, n) \\
U\left(i \omega_{n}\right) & =x(3, n)+i x(4, n) \\
U_{1}\left(\underline{i}_{n}\right) & =x(5, n)+i x(6, n)
\end{aligned}
$$

i.e. $x(1, n)$ is the real part of the measured value of $h$ at frequency $\omega_{n}, x(2, n)$ is the imaginary part of measured value of $h$ at frequency $\omega_{n}, x(3, n)$ is the real part of the measured value of $U$ at frequency $\omega_{n}$, etc. 
In addition, to the data points themselves, the standard errors and the coefficients of correlation in each of these data points is used. Let the standard error in $x(w, n)$ be denoted by $\sigma(m, n)$, and let the coefficient of correlation between $x(j, n)$ and $x(k, n)$ be denoted by $r_{n}(j, k)$. Define, using the notation of Appendix $V$, the elements of a $\bar{P}_{n}$ matrix by:

$$
P_{n}(j, k)=\sigma(j, n) \sigma(k, n) r_{n}(j, k) / \sigma_{0}^{2}
$$

where $\sigma_{0}^{2}$ is an arbitrary constant.

Let the elements $a(j) \quad(j=1,2 \ldots, 6)$ be defined by:

$$
\begin{aligned}
& a(1)=\alpha_{f} \\
& a(2)=\gamma \\
& a(3)=\nu \\
& a(4)=C_{f} \\
& a(5)=c_{q} \\
& a(6)=C_{i}
\end{aligned}
$$

In order to use the procedure outlined in Appendix V, initial estimates of the six parameters are needed. Let the initial estimates of the parameters $a(j)$ be denoted by $a_{0}(j)$.

The only additional quantities which are required are the partial derivatives of $L$ with respect to the measured quantities $x(m, n)$, and the partial derivatives with respect to the parameters $a(j)$. All of these derivatives can be easily calculated, except.for the partial derivatives with respect to $\gamma$ and $\nu$. These derivates can be written as follows:

$$
\frac{\partial L}{\partial \gamma}=-\alpha_{f} X_{f} \frac{\partial F}{\partial \gamma}-C_{f} U \frac{\partial F}{\partial \gamma}
$$




$$
\begin{gathered}
\text { GEAP }-5576 \\
\frac{\partial L}{\partial v}=-\alpha_{f} X_{f} \frac{\partial F_{a v}}{\partial v}-C_{f} U \frac{\partial F_{s}}{\partial v}
\end{gathered}
$$

where:

$$
\begin{aligned}
& \frac{\partial F_{s}}{\partial \gamma}=-i \omega F_{s}^{2} \\
& \frac{\partial F}{\partial v}=-\frac{1}{\nu} \cdot\left[\frac{i \omega v^{2}}{2} F_{s}^{2}+2\left(F_{s}-G_{s}\right) G_{s}\right] \\
& =-\frac{2 i \omega}{v}\left[\begin{array}{lll}
\frac{\nu^{2}}{4} & F_{s}^{2}+\gamma F_{s} G_{s}-\left(\gamma+\frac{v^{2}}{8}\right) & F a v_{s}
\end{array}\right] \\
& \frac{\partial F_{\text {av }}}{\partial \gamma}=\frac{F_{s}^{2}-F_{\text {av }}}{\gamma+v^{2} / 8} \\
& \frac{\partial F_{a v}}{\partial \nu}=\frac{\frac{\nu}{4}\left(2 F_{s}^{2}-F_{a v}-F_{a v} G_{s}\right)+\frac{2 \gamma}{\nu} G_{s}\left(F_{s}-F_{a v}\right)}{\gamma+\nu^{2} / 8}
\end{aligned}
$$

where $G_{S}$ is defined by: $\quad G_{S} \equiv 1-1 \omega$ F $_{S}$

The expression for $F_{\text {av }}$ and $F_{S}$ are given in Section 2.5 .

\subsubsection{Procedure for Fitting of Data}

Using the nomenclature of Appendix $V$, and the previously defined quantities, the quantities $\xi_{\mathrm{kn}}$ and $\psi_{\mathrm{kn}}$ are calculated from the following:

$$
\xi_{\mathrm{ln}}+i \psi_{\mathrm{kn}}=\frac{\partial \mathrm{L}\left(i \omega_{n}\right)}{\partial \mathrm{x}_{\mathrm{kn}}}
$$

where the right hand side is evaluated using measured coordinates and estimated parameters. 
The values for the weights $\sigma_{f n}, \sigma_{g n}$, and $R_{n} \sigma_{f n} \sigma_{g n}$ of Appendix $V$ are then calculated from equations (10), (18), and (19) in the appendix, where the elements of the $\overline{\mathrm{P}}_{\mathrm{n}}$ matrix have been defined above.

The quantities $F_{k n}$ and $G_{k n}$ are calculated from the following:

$$
F_{k n}+1 G_{k n} \doteq \frac{\partial L\left(i \omega_{n}\right)}{\partial a_{k}}
$$

where the right hand side is evaluated using measured coordinates and estimated parameters.

The initial values $f_{\text {no }}$ and $g_{\text {no }}$ are calculated from:

$$
f_{n o}+i g_{n o}=L\left(i w_{n}\right)
$$

where the right hand side is evaluated using measured coordinates, and estimated parameters.

The $\overline{\mathrm{T}}$ matrix and the $\mathrm{C}$ vector are calculated from equations (24) and (25) in Appendix V, and the $\overline{\mathrm{T}}$ matrix is then inverted to obtain the $\bar{Q}$ matrix. The estimate of the values of the parameters is then given by. (27)' in 'Appendix V.'

The value of $S$ is obtained from equation (20), (21), and (22) of Appendix $\mathrm{V}$ and the estimated standard errors and coefficients of correlation are obtained from equations (28), (29), and (30) of Appendix $\dot{\mathrm{V}}$. 
Values: for the calculated curve, $h_{c}(i \omega)$, of the feedback kernel $h(i \omega)$ can be obtained from the following:

$$
h_{c}\left(i \omega_{n}\right)=x(1, n)+i x(2, n)-f_{n}-i g_{n}
$$

where: (from Appendix V - equations (21): and (22), ):

$$
f_{n}=f_{n o}-\sum_{\ell=1}^{L} F_{\ell n}\left(a_{\ell 0}-a_{\ell}\right)
$$

and

$$
g_{n}=g_{n o}-\sum_{\ell=1}^{L} G_{\ell n}\left(a_{\ell 0}-a_{\ell}\right)
$$

where: $L=$ the number of parameters.

The estimated standard error in the real. part: of $h$ (due to uncertainties in the: parameters) at: frequency: $\omega_{n}$ is given by:

$$
\sigma_{i}=\left[\sum_{n=1}^{L} \quad \sum_{k=1}^{L} F_{j, n} F_{k n} Q_{j k}\right]^{1 / 2}
$$

The estimated standard error in the imaginary part: of h is given by:

$$
\sigma_{i}=\left[\sum_{j=1}^{\mathrm{E}} \sum_{\mathrm{k}=1}^{\mathrm{L}} \mathrm{G}_{\mathrm{jn}} G_{\mathrm{kn}} Q_{\mathrm{j} \mathrm{k}}\right]^{1 / 2}
$$

The callculated value of: h(i $\left(w_{n}\right)$; which correspond's; to the measured value: of: $h\left(h_{i}\right)$, , i..e. the "adjusted value: of: the coordinate: (see: equation (15)) and (15,-a); of: Appendix: V)) is; given: by:

$$
\left.h_{m !}\left(i w_{n}\right)\right\rangle=x(1, n)-\lambda_{n} \sum_{j=1}^{K} P_{n}(1, j) \xi_{j n}-v_{n:} \sum_{j=1}^{K} P_{n}(1, j) \psi_{j n}
$$




$$
+i \cdot\left\{x(2, n)-\lambda_{n} \sum_{j=1}^{K} P_{n}(2, j) \xi_{j n}-v_{n} \sum_{j=1}^{K} P_{n}(2, j) \psi_{j n}\right\}
$$

where $\mathrm{K}=$ the number of coordinates and where $\lambda_{\mathrm{n}}$ and $\nu_{\dot{n}}$ are given by equations (16) and (17) of Appendix $V$, and the $f_{n}$ and $g_{n}$ which are used in these equations are defined above.

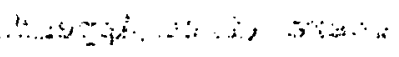

Although the value of $h_{m}\left(i \omega_{n}\right)$, (see above), should. lie on the calculated curve for $h\left(i \omega_{n}\right)$, it will not, in general, coincide with the value $h_{c}\left(i \omega_{n}\right)$ are given by equation (5.2-1), but rather will lie on the curve drawn between the points $h\left(i \omega_{n}\right)$.

The Doppler energy coefficient, $n$, is obtained from the following expression:

$$
n=\frac{a(1)-c_{p}}{\left(a(2)+a^{2}(3) / 8\right) A_{f}}
$$

where $C_{p}$ is the calculated power dependent portion of the fuel and fuel clad axial expansion effects (see Section 2.4).

The square of the estimated standard error, $\sigma_{\eta}$ in $\eta_{i}$ is calculated from the following:

$$
\begin{aligned}
& \sigma_{n}^{2}=\frac{\sigma^{2}}{\left[a(2)+a^{2}(3) / 8\right]^{2}}\left[\left(\frac{1}{A_{f}}\right)^{2} Q_{11}+n^{2} \ddot{Q}_{22}+n^{2} \frac{a^{2}(3)}{16} Q_{33}\right. \\
& \left.\quad \therefore 2 \eta Q_{12} / A_{f}-\eta a(3) Q_{13} / 2 A_{f}+n^{2} \cdot \frac{a(3)}{2} Q_{23}+\sigma_{C}^{2} \frac{1}{\sigma^{2} A_{f}^{2}}\right]
\end{aligned}
$$

where $\sigma_{C}^{2} i s$ the estimated standard error in $C_{P}$, and the $Q(i, j)$ are elements of the $\bar{Q}$ matrix. 
GEAP -5576

In the event that it is desired to treat one of the parameters, say $a(l)$, as known, the $F_{n}$ and $G_{n}$ vectors are reduced to 5 element vectors by removing the $\ell-t$ h element (this reduces $T$ and $Q$ to $5 \times 5$ matrices, and $Y$ and $C$ to a 5 element vector).

The term $\sigma^{2}(\ell) \mathrm{F}_{\ell \mathrm{n}}^{2}$ is added to $\sigma_{\mathrm{fn}}^{2}$, the term $\sigma^{2}(\ell) G_{\ell \mathrm{n}}^{2}$ is added to $\sigma_{\mathrm{gn}}^{2}$, and the term $\sigma^{2}(\ell) F_{\ell n} G_{\ell n}$ is added to $R_{n} \sigma_{f_{n}} \sigma_{g n}$, where $\sigma(l)$ is the estimated standard error in $a(l)$. The calculation then proceeds as before.

\subsubsection{Effects of Biased Errors}

The standard errors in the reactivity coefficients which have been discussed in the previous sections include only the "random" errors in the measurements. Uncertainties in instrument calibration (i.e. absolute power, flow, or temperature) have little effect on the frequency response parameters. These uncertainties do affect the reactivity coefficients, however, and must be included in an estimate of the nver-all uncertainty in the coefficients.

If it is assumed that there is equal probability that the true mean value lies above or below the calibrated value, and that the over-a11 uncertainty in a parameter which depends on more than one measurement can be approximated by propagating the biased errors as if they were random, the uncertainty in the reactivity coefficients can be calculated as follows.

Using the notation of the previous section, the over-all uncertainty $\bar{\sigma}_{1}$ in. the fuel power coefficient, $a_{1}$, is obtained from the following:

$$
\left(\frac{\bar{\sigma}_{1}}{a_{1}}\right)^{2}=\left(\frac{\sigma_{1}}{a_{1}}\right)^{2}\left(\frac{u_{p}}{p_{0}}\right)^{2}+\left(\frac{\sigma_{k}}{k_{0}}\right)^{2}
$$

where $\sigma_{1}$ is the standard random error in $a_{1},\left(\sigma_{p} / p_{0}\right)$ is the fractional error in the absolute phiwer level, and $\sigma_{k} / k_{0}$ is the fractional error in the absolute reactivity oscillation. 
The over-all uncertanties $\bar{\sigma}_{j}(j=4,5,6)$ in the temperature coefficients $a_{j}(j=4,5,6)$ defined in the previous section are obtained from the following:

$$
\left(\frac{\bar{\sigma}_{j}}{a_{j}}\right)^{2}=\left(\frac{\sigma_{i}}{a_{j}}\right)^{2}+\left(\frac{\sigma_{T}}{T_{0}}\right)^{2}+\left(\frac{\sigma_{k}}{k_{o}}\right)^{2} \quad(j=4,5,6)
$$

where $\sigma_{j}$ is the random error described in the previous section, where. $\left(\sigma_{k} / k_{o}\right)$ is defined above, and where $\left(\sigma_{T} / T_{0}\right)$ is the fractional error in the absolute coolant temperature measurement.

Since the Doppler energy coefficient, $n$; is determined from the calculated value of the fuel power coefficient, $a_{1}$, the over-all uncertainty, $\bar{\sigma}_{n}$ in $\eta$ must include the uncertainties in the absolute power and reactivity measurements. Thus,

$$
\left(\frac{\bar{\sigma}_{n}}{\eta}\right)^{2}=\left(\frac{\sigma_{n}}{\eta}\right)^{2}+\left(\frac{\sigma_{\mathrm{p}}}{\mathrm{P}}\right)^{2}+\left(\frac{\sigma_{\mathrm{k}}}{\mathrm{k}_{0}}\right)^{2}
$$

where $\sigma_{\eta}$ and $\eta$ are defined in previous sections and where $\left(\sigma_{P} / P_{0}\right)$ and $\left(\sigma_{k} / k_{0}\right)$ are defined above.

\subsubsection{Expected Results}

The SEFOR plant has a regulating device which (when in use) automaticaliy adjusted the air flow rate in the air blast heat exchanger in order to keep the cold leg inlet temperature of the intermediate heat exchanger (IHX) constant. In order to determine the effect of this device on the reactivity feedback during a conventional oscillator test, the power-reactivity transfer function was calculated with automatic control. (constant IHX secondary inlet temperature) and without automatic 
control (constant air flow). The reactivity feedback model described in Section 2.5.1 and the model for the coolant temperature response that is described in Appendix III were used. The results are shown in the following figures.

The automatic control "smooths out" the power-reactivity transfer function at $5 \mathrm{MW}$ and $30 \%$ flow, (see Figures 5.2-1, 5.2-2, and $5.2-11,5.2-12$ ), and reduces* the coolant temperature feedback at very low frequencies $(0.001 \mathrm{rad} / \mathrm{sec})$. However, the relative magnitude of coolant and fuel temperature feedback in the frequency range of interest ( 0.01 to $-0.2 \mathrm{rad} / \mathrm{sec})$ are comparable in the two cases (i.e., the amplitude and phase of the power-reactivity transfer functions are similar over the frequency range 20.01 to $20.2 \mathrm{rad} / \mathrm{sec})$, and it is expected that the Doppler effect will be determined with the same accuracy in the conventional oscillator. tests regardless of whether or not the IHX cold leg inlet temperature is held constant. The effect of different Doppler and coolant coefficients upon the power-reactivity transfer functions is illustrated in Figures 5.2-1 through 5:2-10, where the coolant and Doppler coefficients are simultaneously increased and decreased by $20 \%$. The effect of a change in the Doppler coefficient alone is more clearly illustrated in Section 5.4.4.

* This may be observed by noting that the power-reactivity transfer functions with constant IHX secondary inlet temperature have larger amplitudes and smaller phase angles than the comparable transfer functions with constant flow at $0.001 \mathrm{rad} / \mathrm{sec}$. This is the same trend that occurs when the coolant reactivity effect is zero - see Section 5.4.4. 


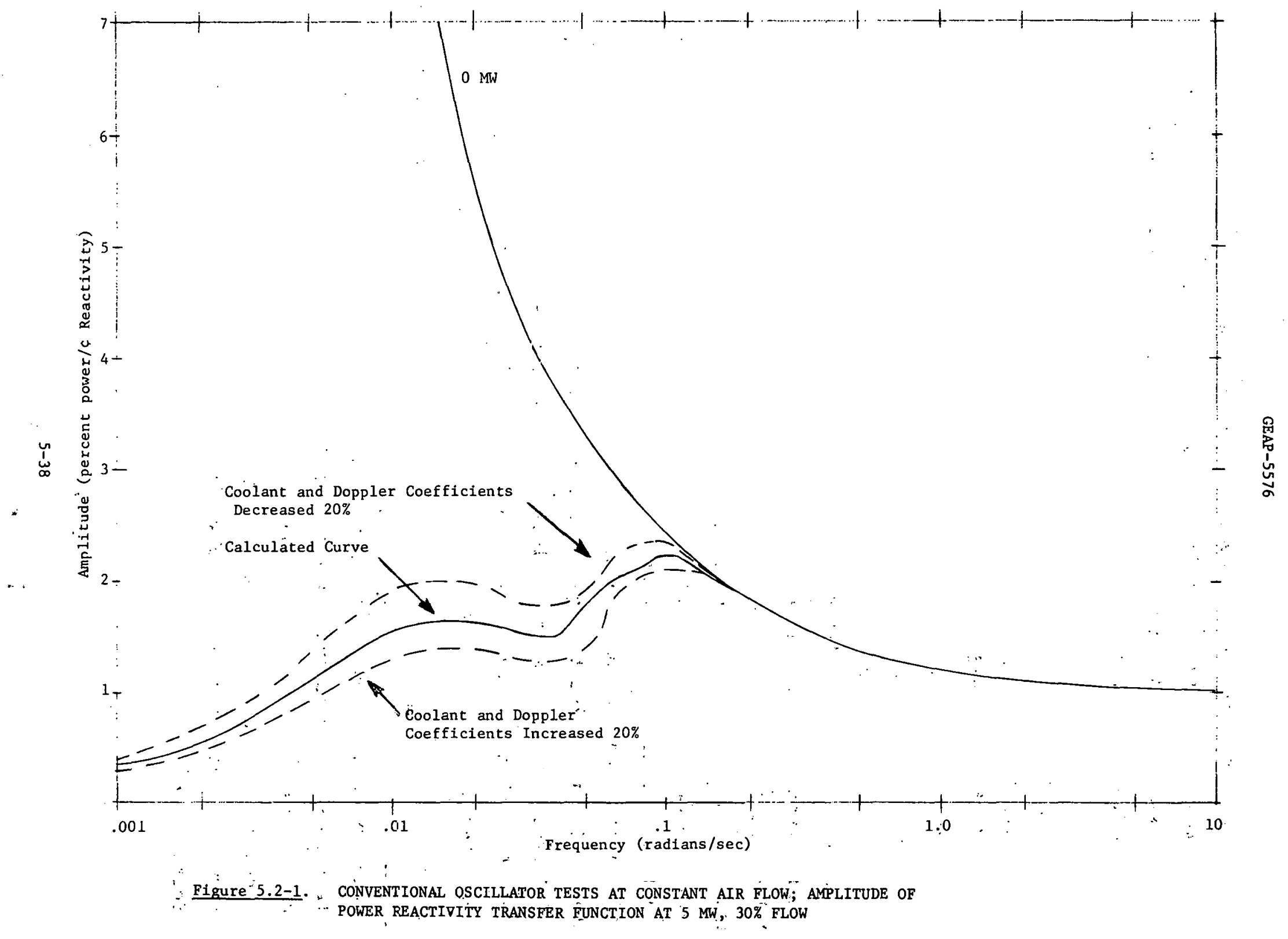




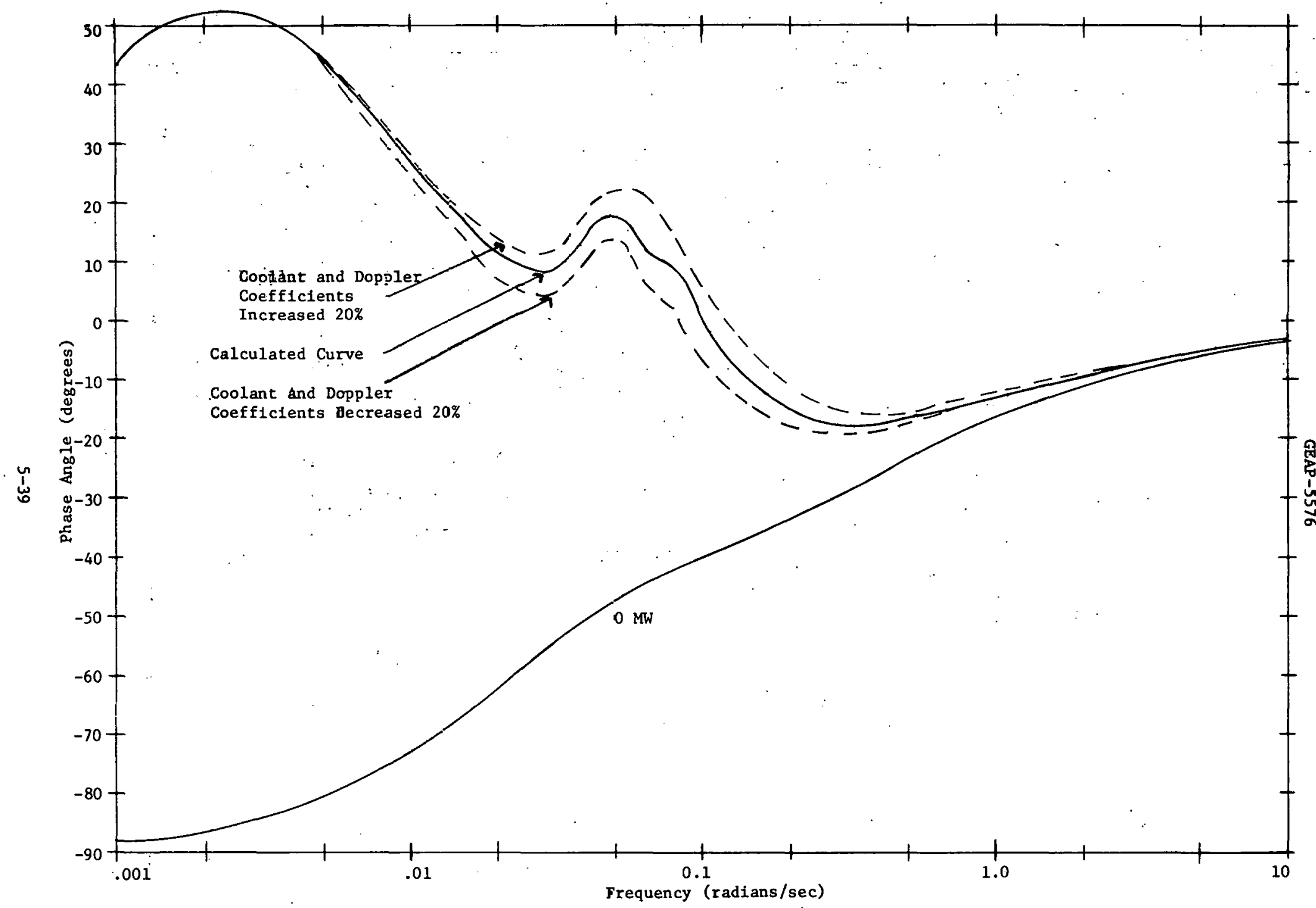

F1gure 5.2-2. CONVENTIONAL OSCILLATOR IESTS AT CONSTANT AIR FLOW; PHASE OF POWER REACTIVTTY TRANSPER FUNCTION AT 5 MW, 30\% FLOW 


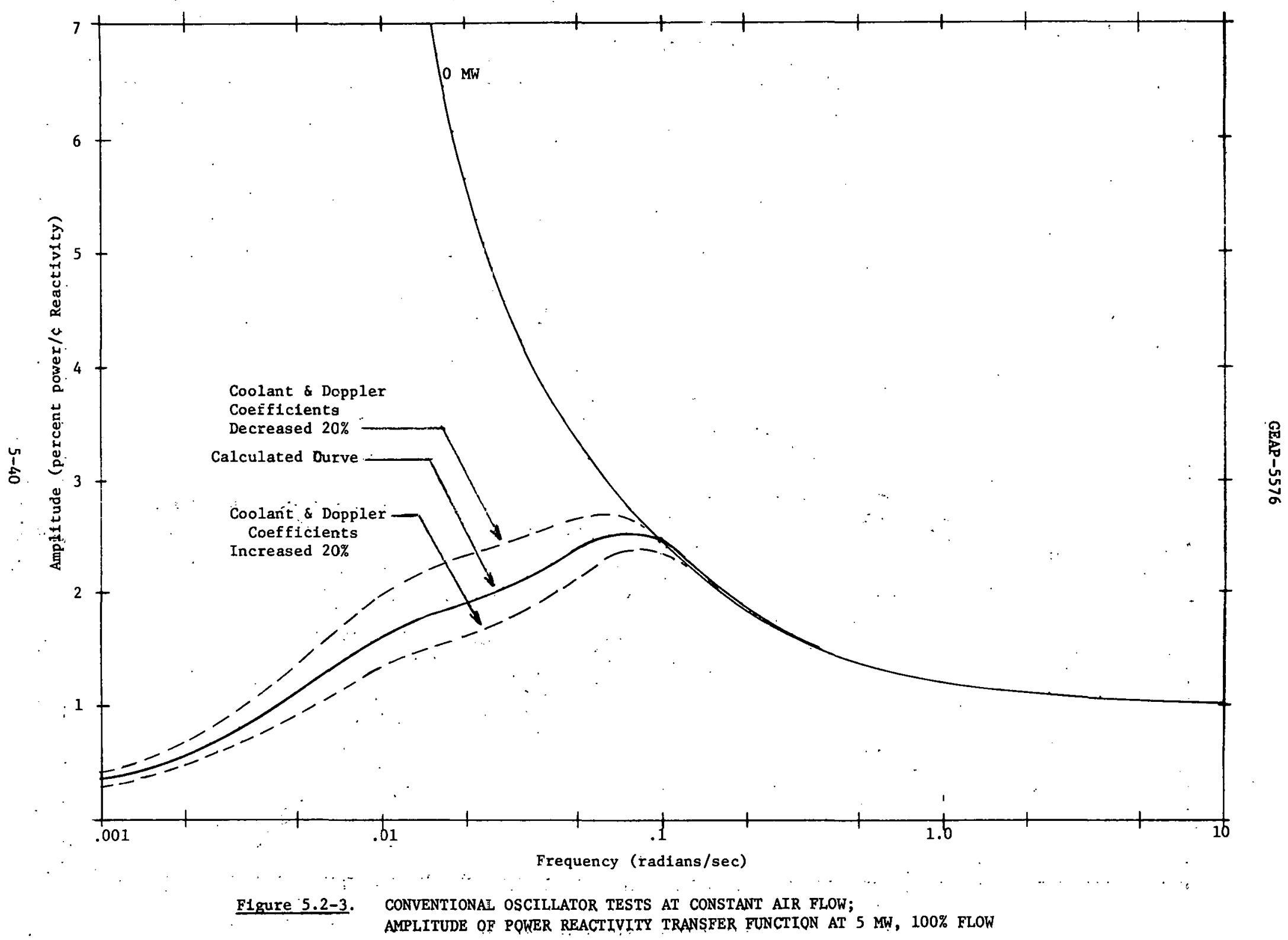




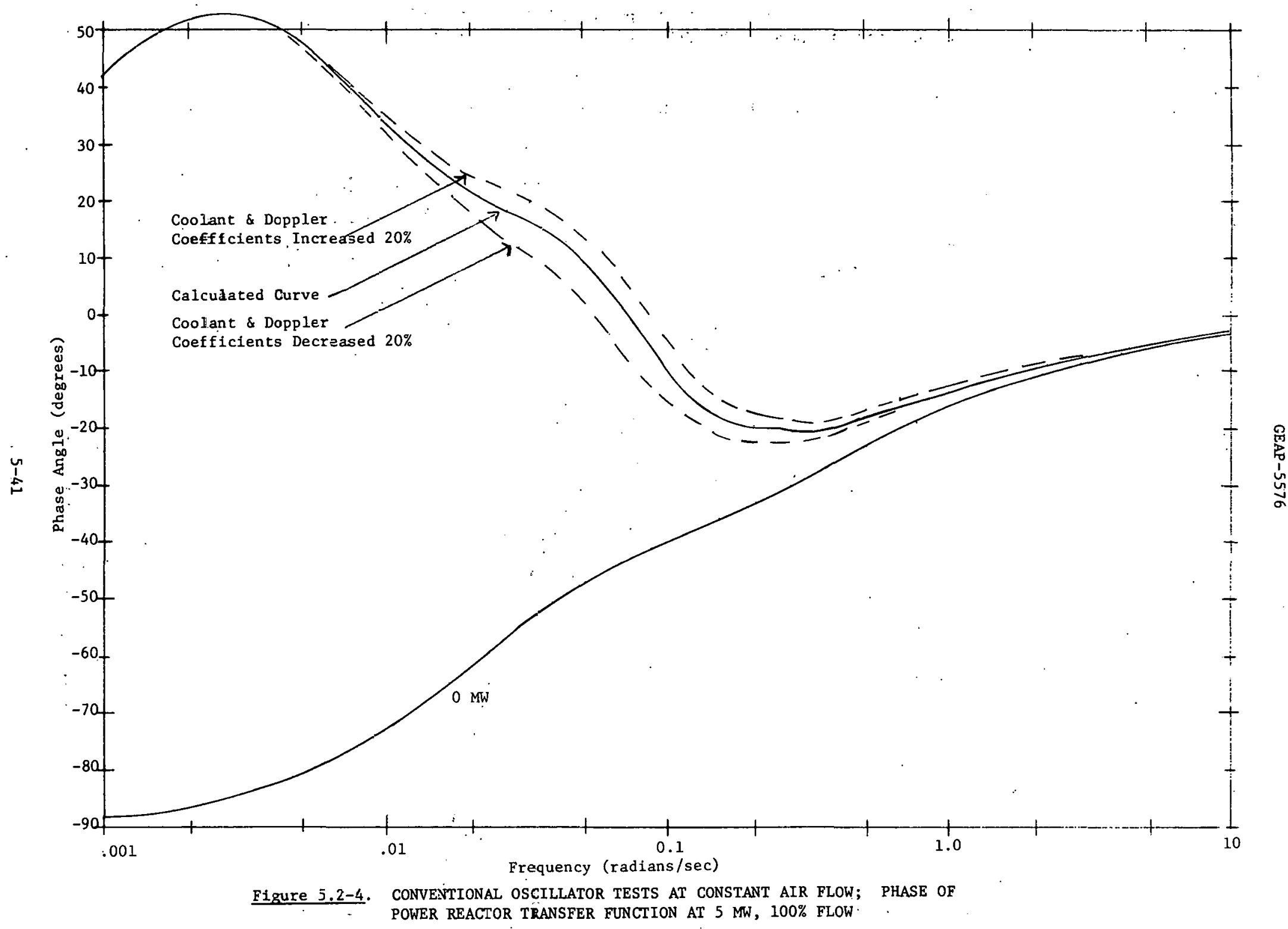




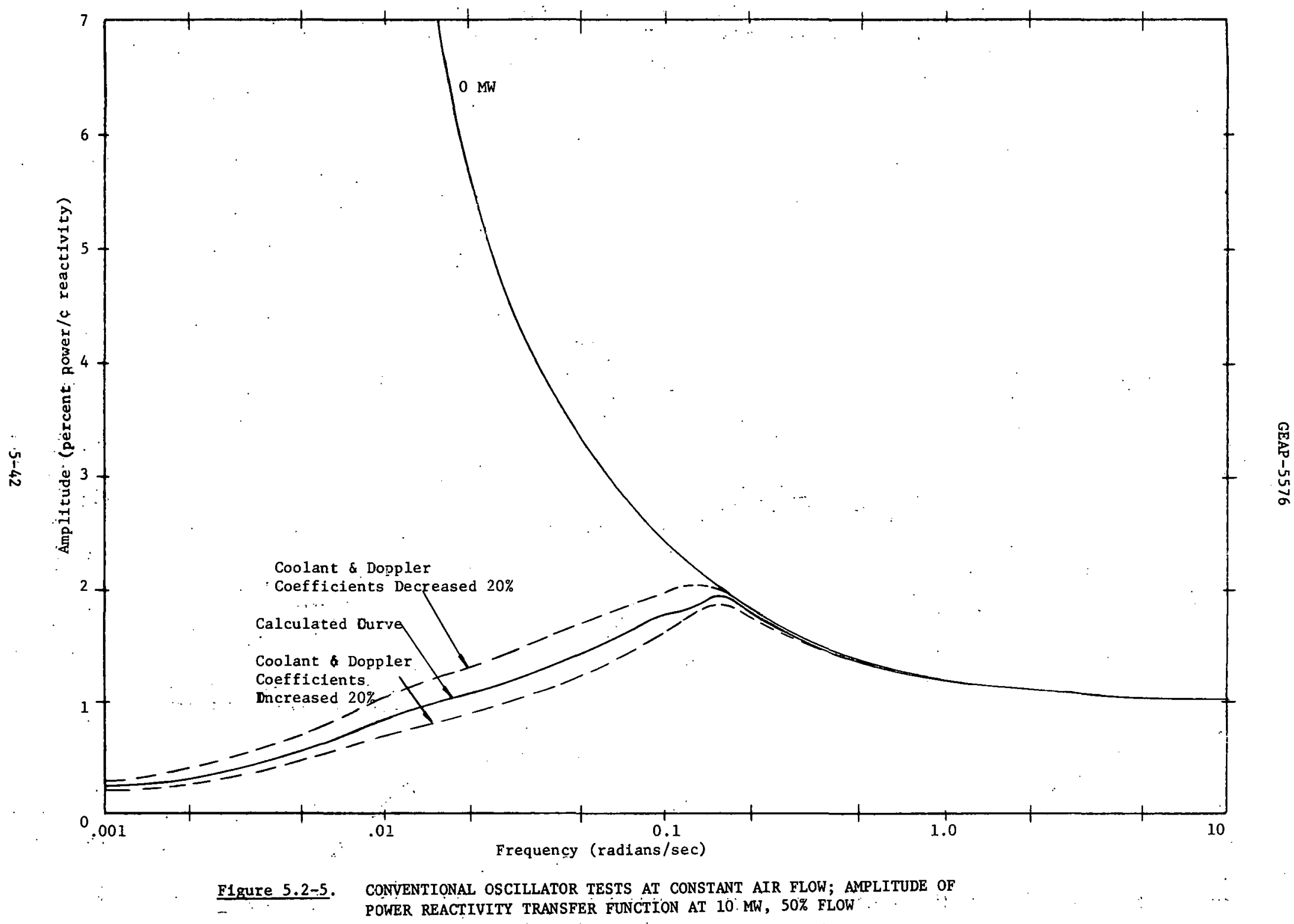




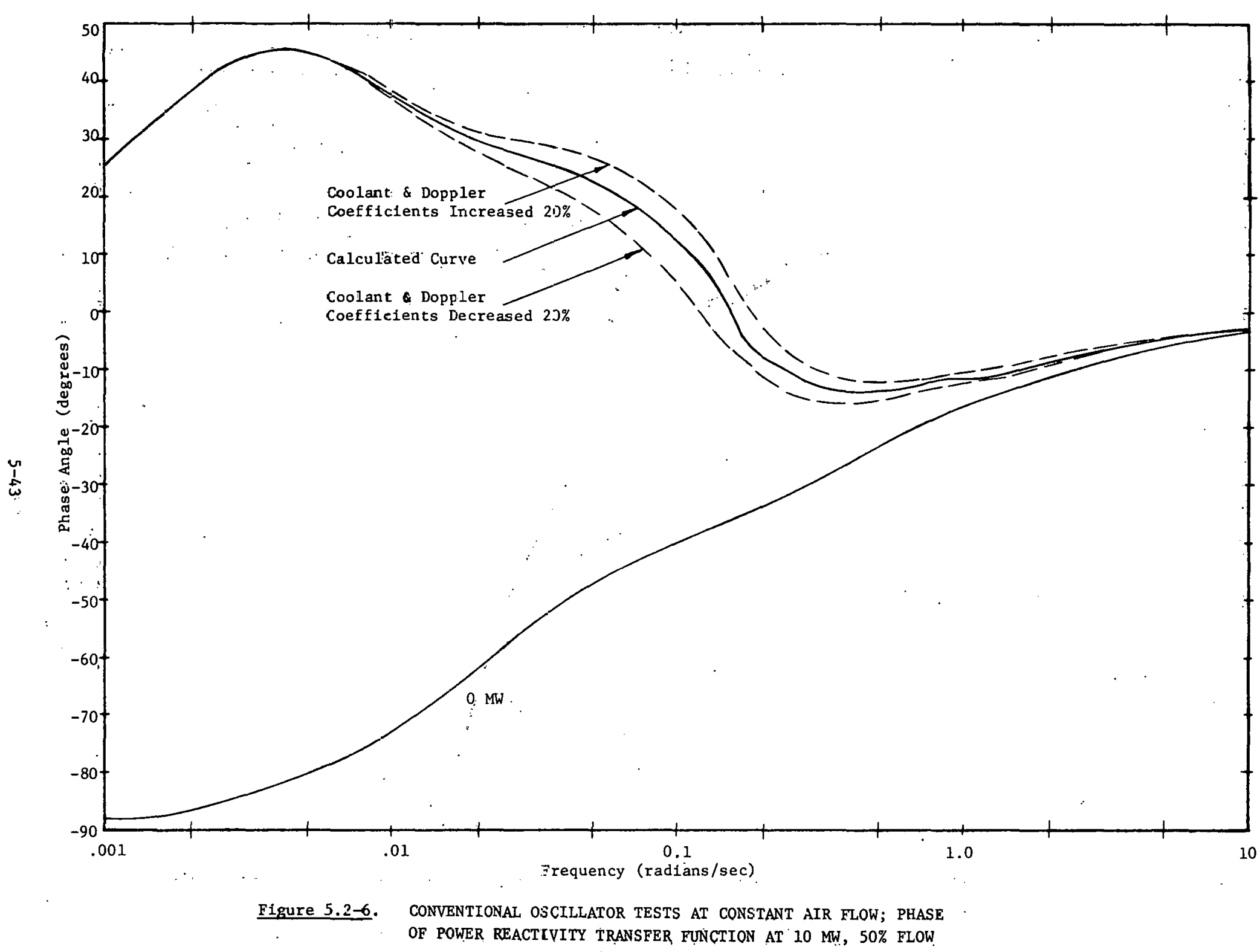




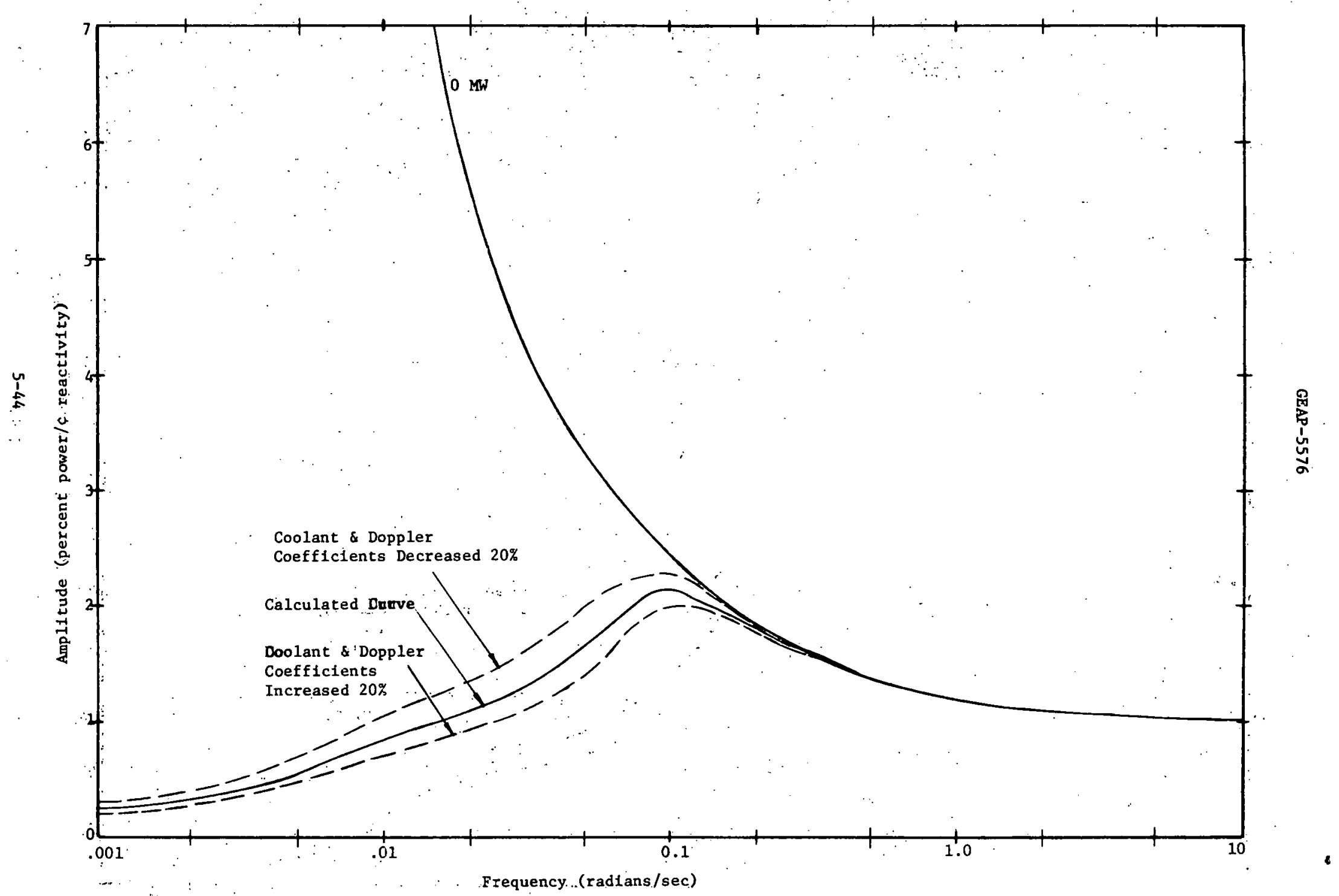

FIgure 5.2-7. CONVENTIONAL OSCILLATOR TESTS AT CONSTANT AIR FLOW; AMPLITUDE OF POWER REACTIVITY TRANSFER FUNCTION AT $10 \mathrm{MW}, 100 \%$ FLOW 


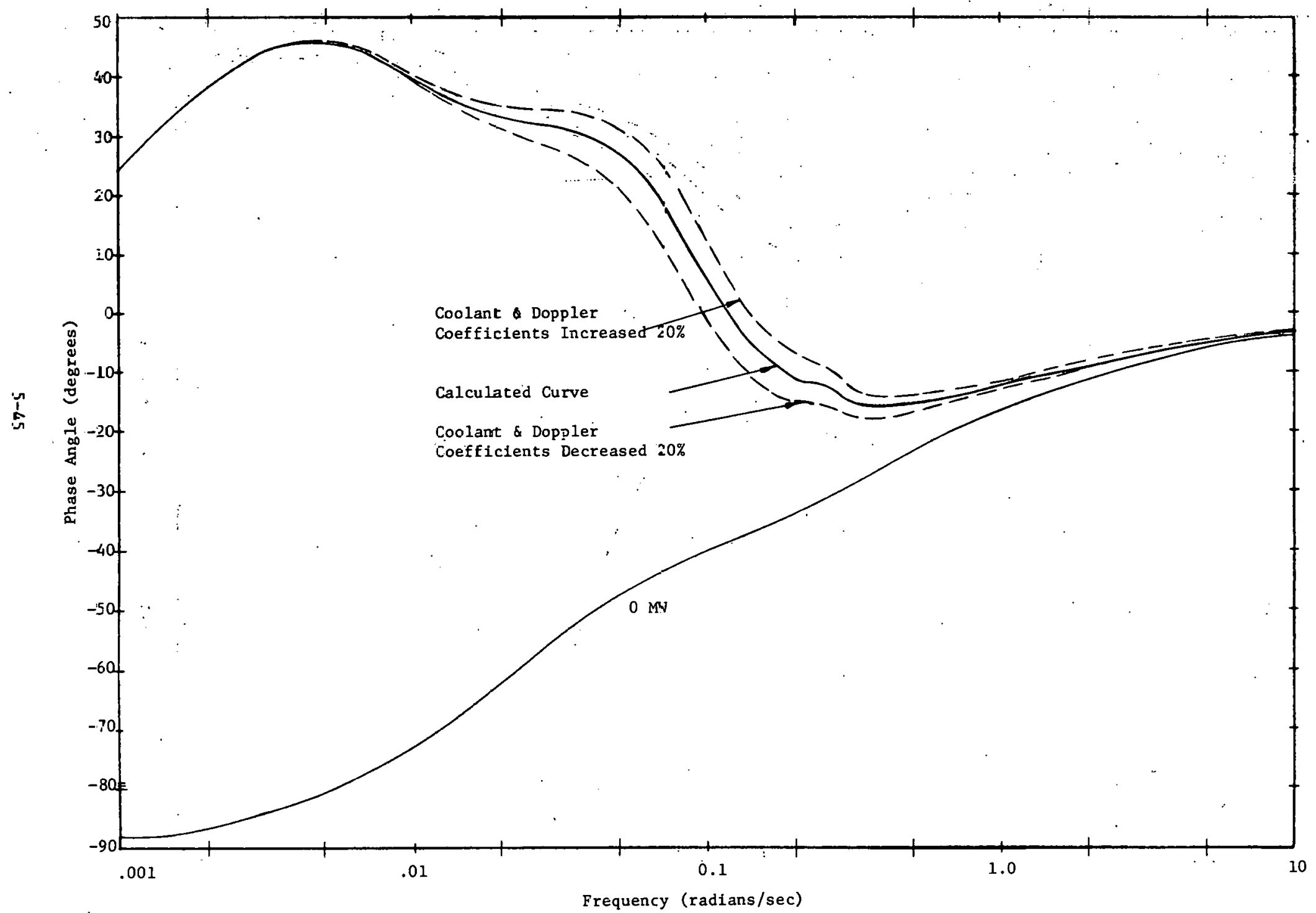

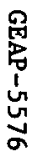

Flgure 5.2-8. CONVENTIONAL OSCILLATOR TESTS AT CONSTANT AIR FLOW; PHASE OF POWER REACTIVITY TRANSFER FUNCTION AT $10 \mathrm{MW}, 100 \%$ FLOW 


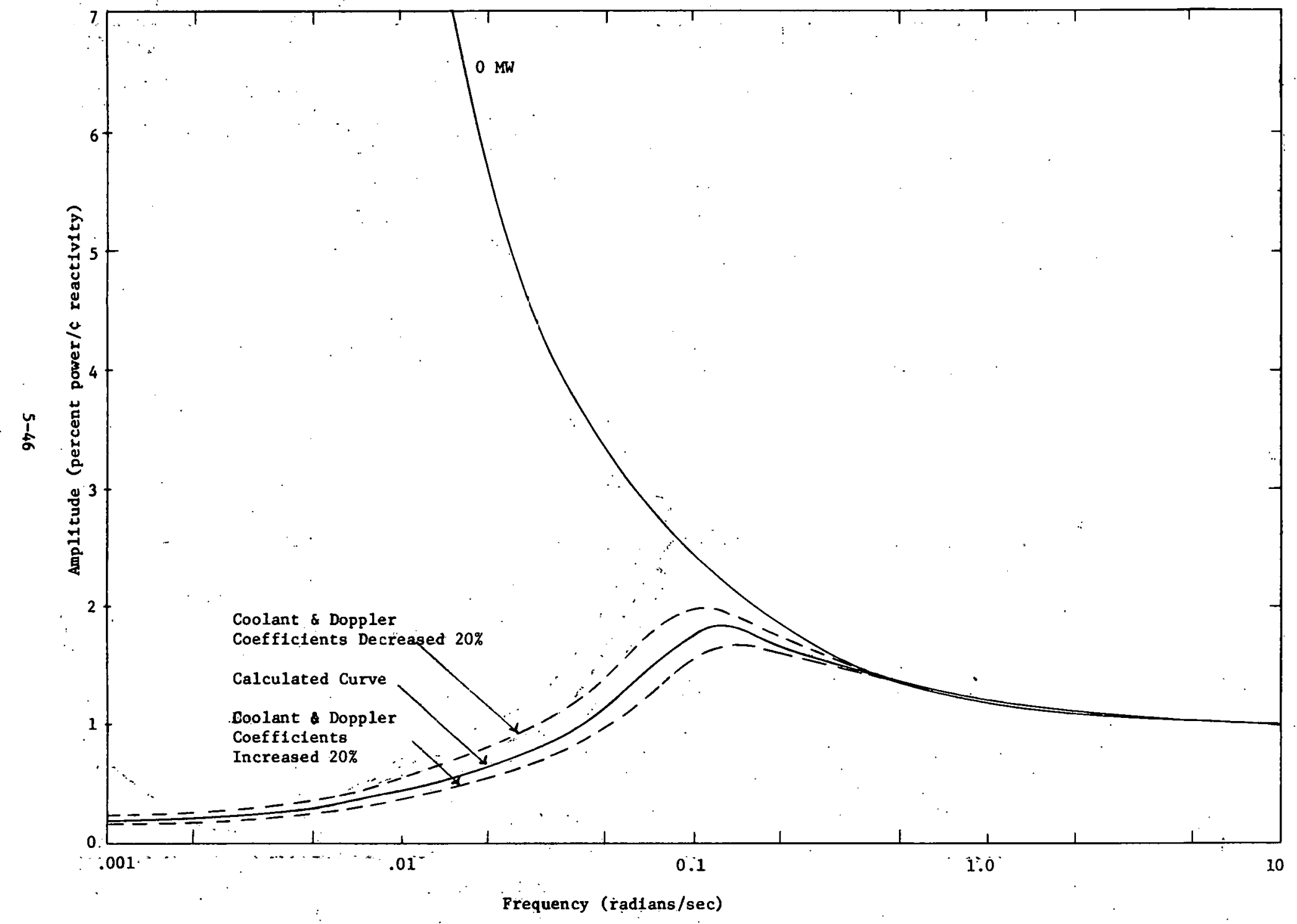

P1gure 5.2-9. CONVENTIONAL OSCILLATOR TESTS AT CONSTANT AIR FLOW; AMPLITUDE OF POWER REACTIVITY TRANSFER FUNCTION AT. $20 \mathrm{MW}, 100 \%$ FLOW 


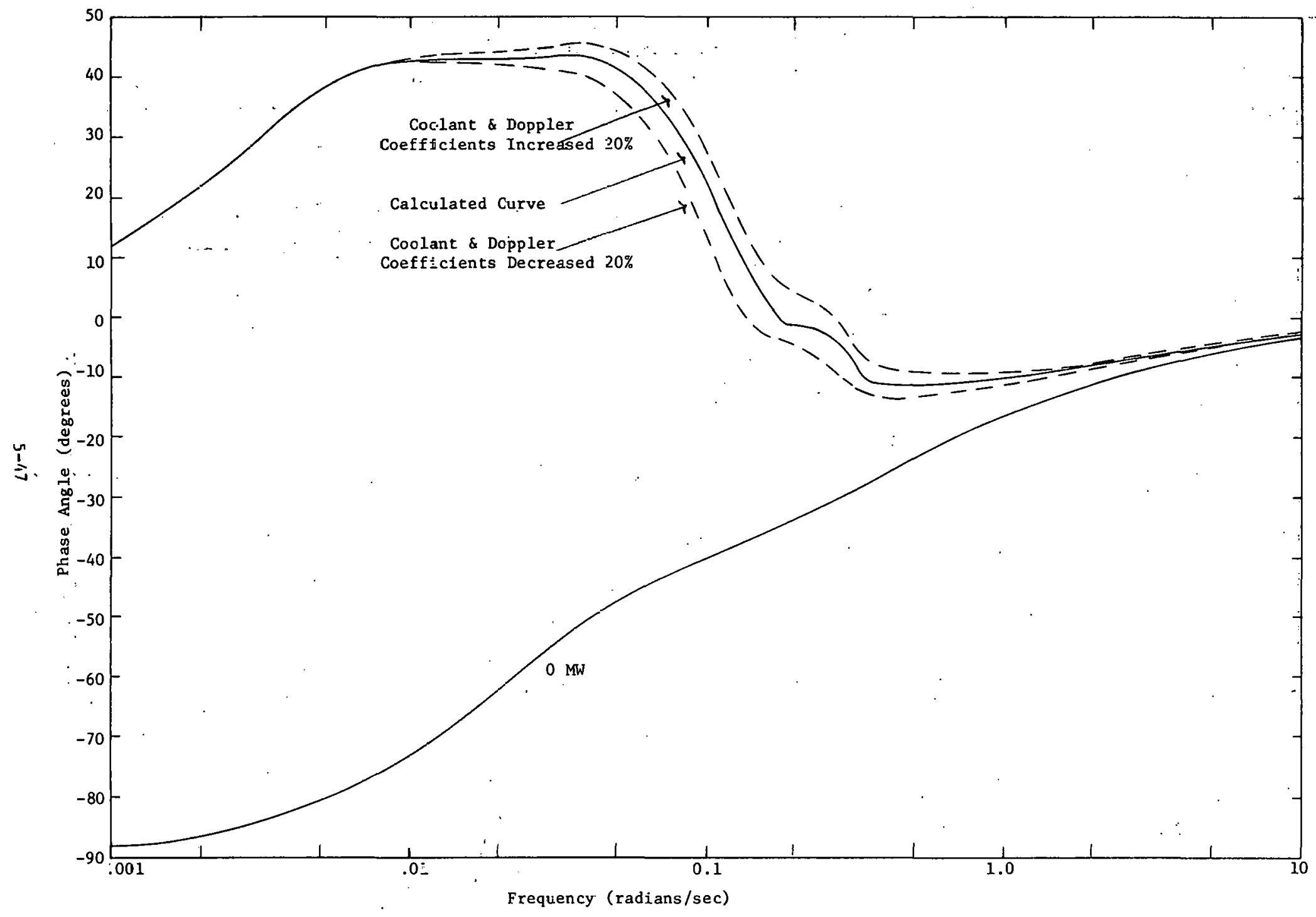

F1gure 5.2-10. CONVENTIONAL OSCILLATOR TESTS AT CONSTANT AIR FLOW; PHASE OF POWER REACTIVITY TRANSFER FUNCITON AT $20 \mathrm{MW}, 100 \%$ FLOW 


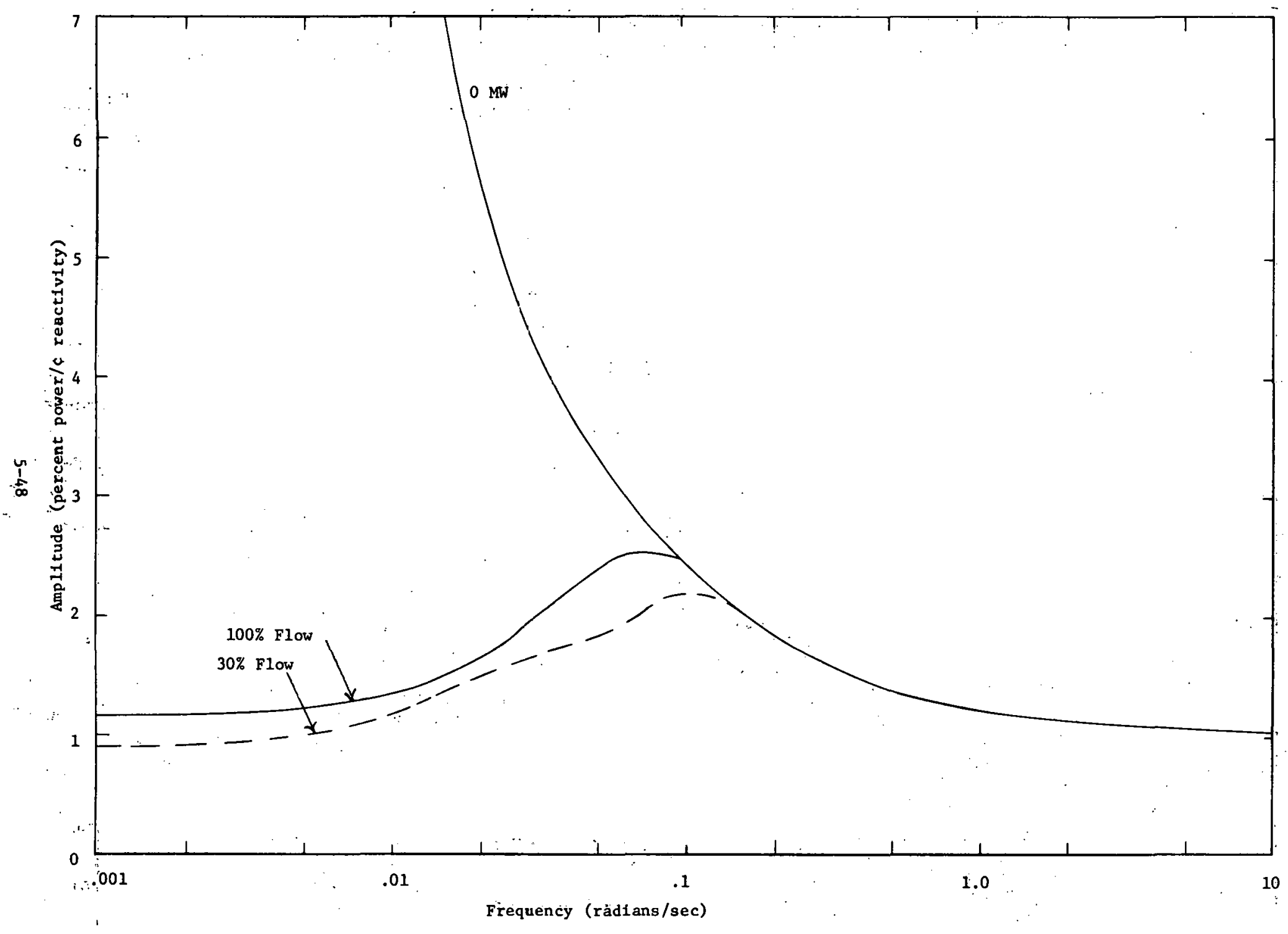

Figure 5.2-11. CONVENTIONAL OSCILLATOR TESTS AT CONSTANT IHX SECONDARY INLET TEMPERATURE; AMPLITUDE OF POWER-REACTIVITY TRANSFER FURCTION AT $5 \mathrm{MW}$ 


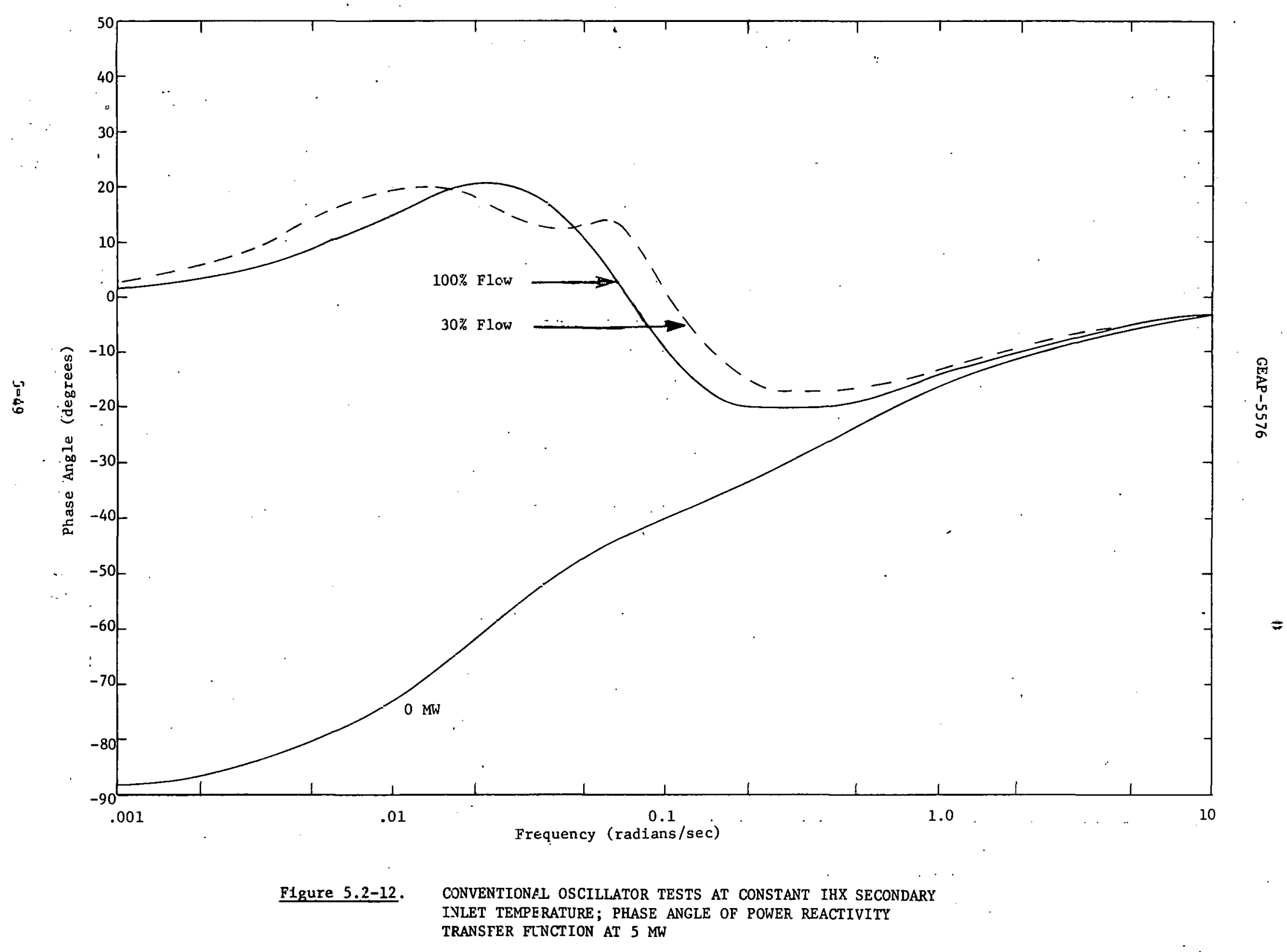




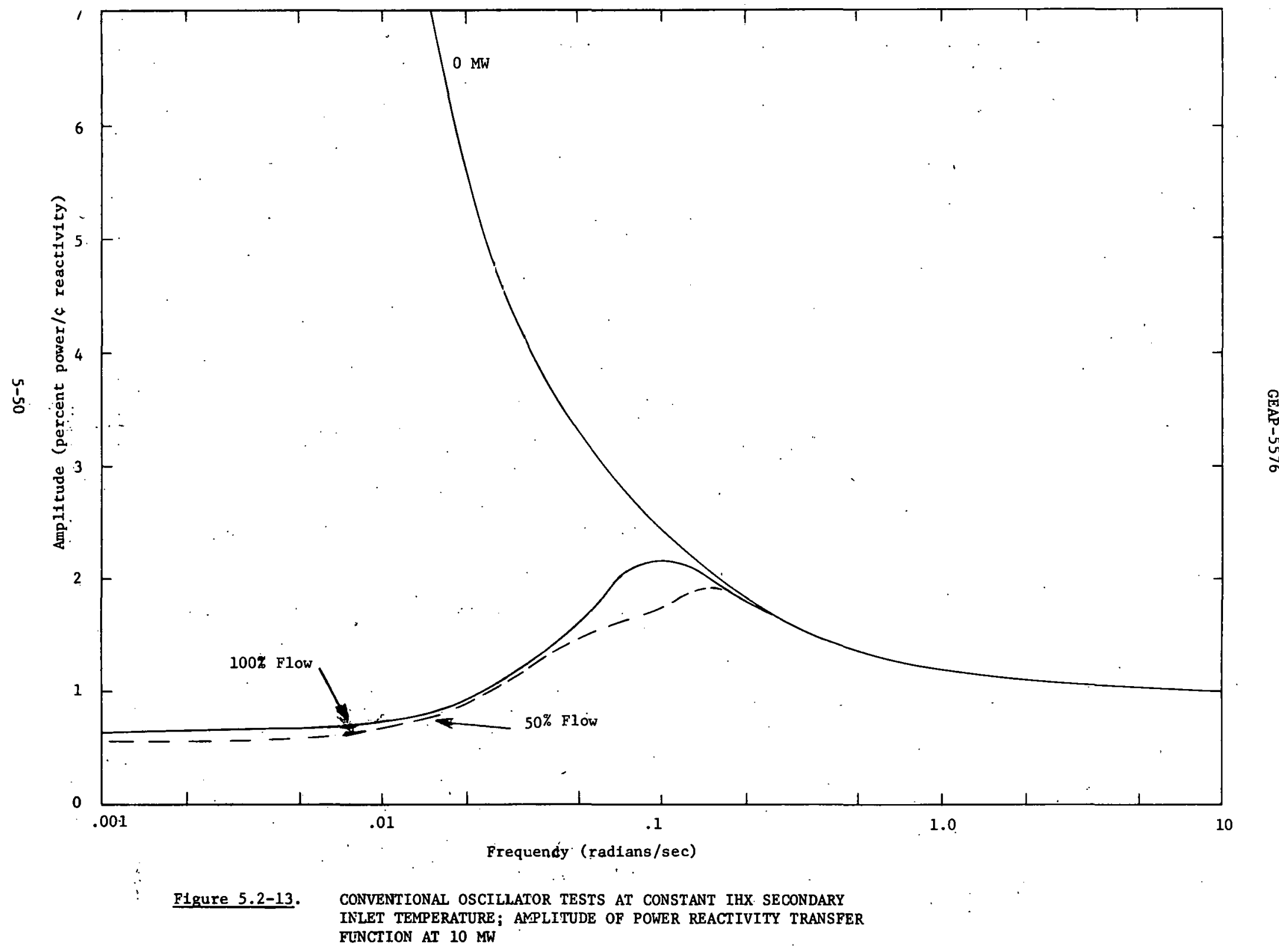




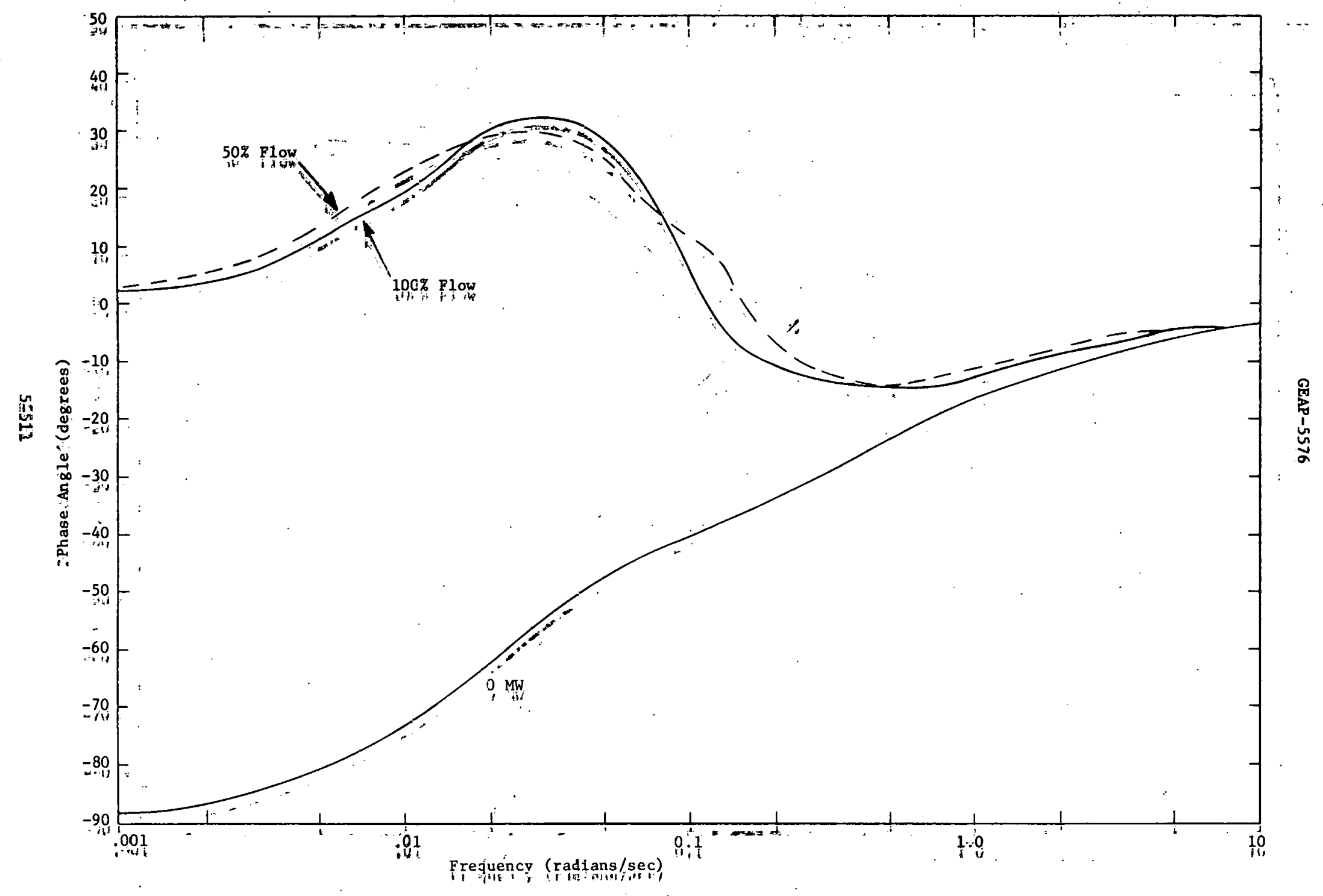

F1gure 5.2-14. CONFENTIONAL OSCTLLATOR TESTS AT CONSTANT IBX SECONDARY INLET TEMPERATURE PHASE ANGLE OE POWER REACTIVTY"TRANSER FUNCTON AT $\mathrm{YO} M \mathrm{MW}$ 


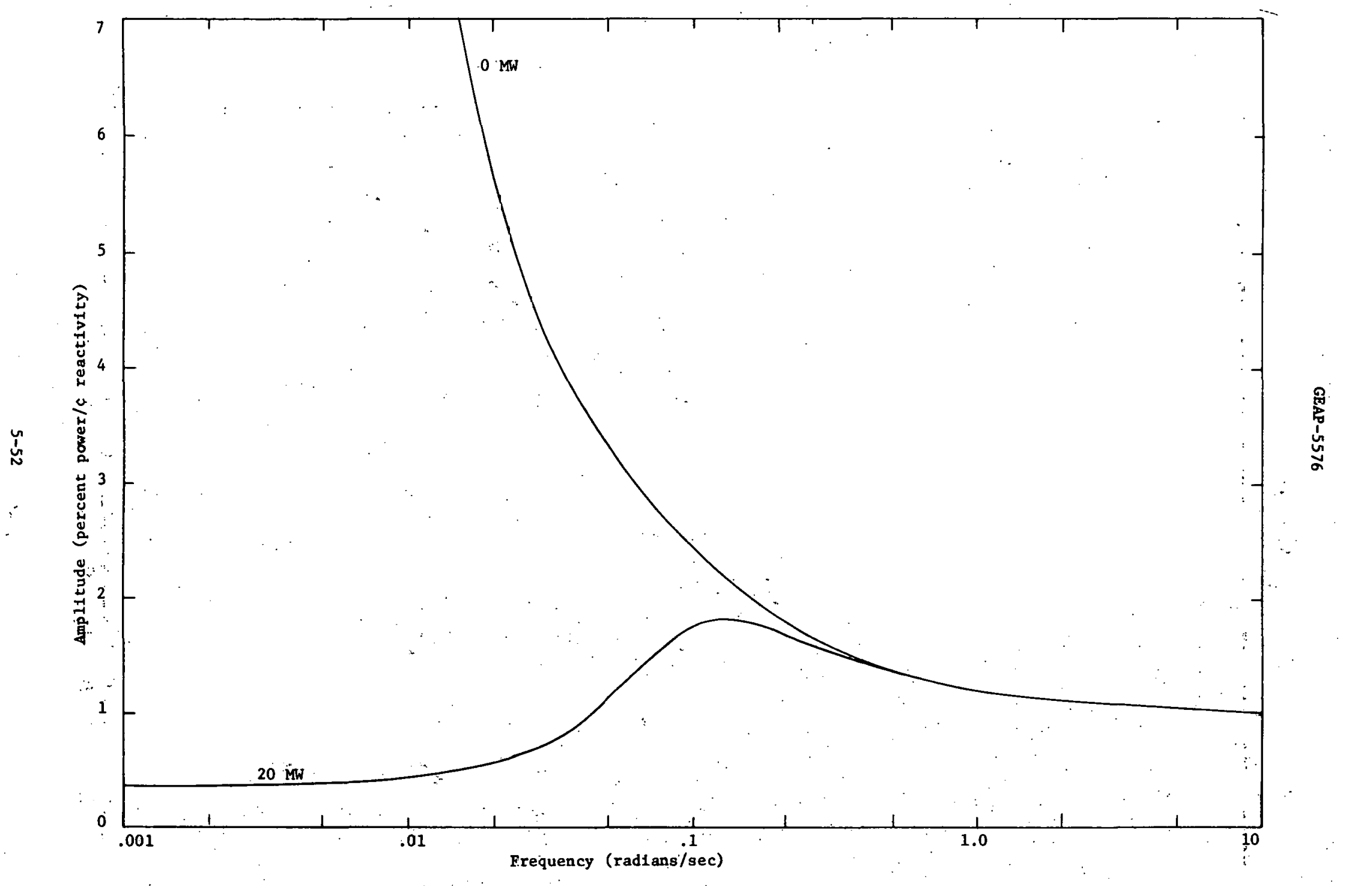

Figure 5.2-15. CONVENTIONAL OSCILLATOR TESTS AT CONSTANT IBX SECONDARY DYLBT TEMPERATURE; AMPLITUDE OF POWER RBACTIVITY TRANSFBR FUNCTION AT $20 \mathrm{MW}, 100 \%$ FLOW 


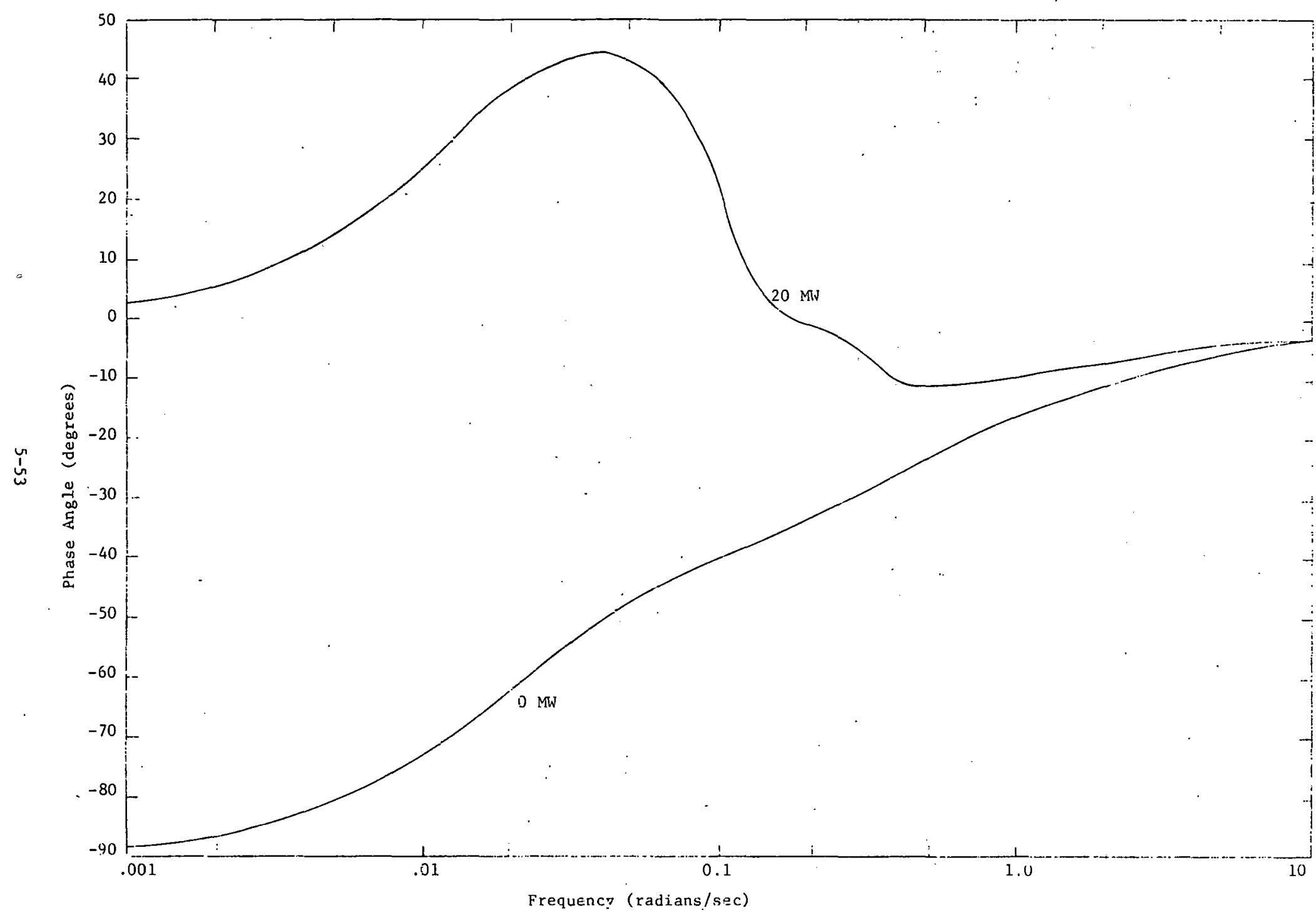

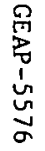

Figure 5.2-16. CONVENTIONAL OSCILLATOR TESTS AT CONSTANT IHX SECONDARY

INLET TEMPERATURE; PHASE ANGLE OF POWER REACTIVITY

TRANSFER FUNCTLON AT $20 \mathrm{MW}, 100 \%$ FLOW 


\subsection{FLOW AND REACTIVITY OSCILLATIONS}

\subsubsection{Objective}

The coolant flow rate in the primary loop, the coolant flow rate in the secondary loop, and the reactivity (poison rod) will be separately oscillated at a few frequencies at the mean power levels, flow rates, and coolant temperatures which will be used in the balanced oscillator tests. The purpose of these oscillations is to provide information which will be used to predict the relative phase angles and amplitudes required for the balanced oscillator tests. Since a number of the conventional and balanced oscillator tests are performed with the same mean reactor conditions, additional reactivity oscillations will not be required at these test conditions.

The flow oscillations will also serve to demonstrate reactor response to flow (temperature) oscillations.

\subsubsection{General Procedure and Test Conditions}

The general procedure is outlined in Section 5.0.2. The signals of primary, interest are:

1. The reactor power

2. The oscillator rod (reactivity) signal - if oscillated

3. The primary coolant flow signals from the instrumented fuel assemblies and from the magnetic flow meter in the primary system - if oscillated

4. The secondary coolant flow signals, which can be recorded on one channel of the 8-channel oscillograph (these signals are not recorded in the data acquisition system) - if oscillated

5. The inlet and outlet coolant temperature signals from the, instrumented fuel assemblies. 
The test conditions for the flow and reactivity oscillations correspond to the test conditions for the first and second ballanced oscillator tests and are shown in Tabile 5-3i. The primary flow, and the secondary flow should be separately oscillated at these conditions. The reactivity should not be oscillated at those test conditions; which are the same as the conventional oscillator test conditions. These are indicated in Table 5-3.

The four suggested frequencies of oscillation are $0.025 ; 0.0110$, 0.004 , and 0.0016 .cps. In order to correctly normallize the worth of: the: poison rod it may be desirable to oscillate the rod at the additional. frequency of 0.5 eps.

TABLE: $5,-3$

\section{FLOW AND REACLIVITY USCIELATIONS}

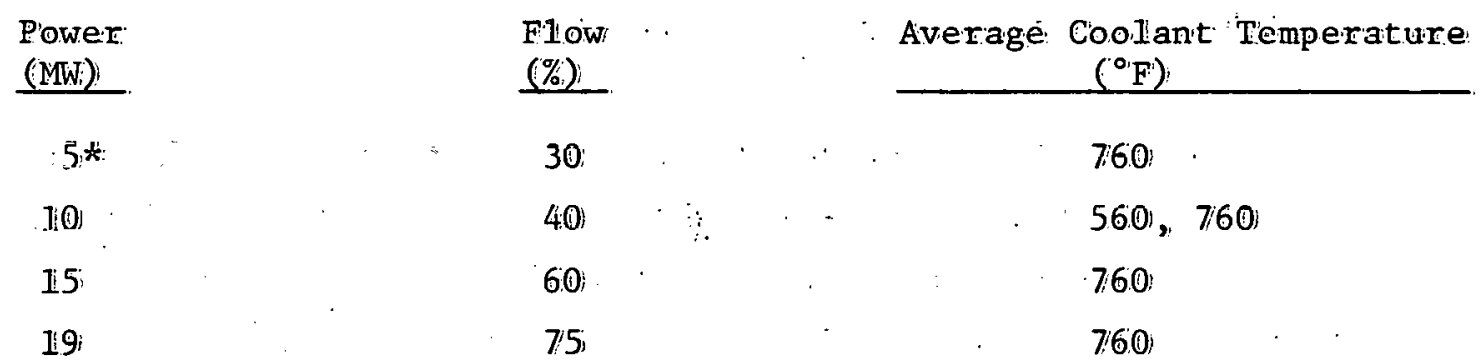

* Flow Oscillations Only - the reactivity oscillations are obttained from the conventional oscilliator test: 


\subsubsection{Data Collection and Analysis}

- The data will be collected and analyzed as described in steps 1 through 3 of Section 5.0.3 to determine nine transfer functions; three from the reactivity oscillation at constant, flow:rate, three from the $\therefore$.. primary flow oscillation at constant reactivity and secondary flow rate, and three from the secondary flow oscillation at constant.reactivity and primary flow rate.

The three transfer functions to be determined from the reactivity oscillation are the response of the fractional power oscillator. $\left(\Delta \mathrm{P} / \mathrm{P}_{\mathrm{o}}\right)$; the response of the core inlet temperature $\left(\Delta \mathrm{T}_{i}\right)$; and the response of the core outlet temperature $\left(\Delta T_{0}\right)$ to the reactivity : oscillation $\Delta \mathrm{K}$. Let these transfer functions be denoted by $G_{1}, G_{2}$, and $G_{3}$, respectively.

The three transfer functions to be determined from the primary coolant flow osciliation are the response of $\Delta \mathrm{P} / \mathrm{P}, \Delta \mathrm{T}_{1}$, and $\Delta \mathrm{T}_{0}$ to the primary fractional flow oscillation $\Delta \mathrm{v}_{1} / \mathrm{v}_{10^{\circ}}$ Let these transfer functions be denoted by $F_{1}, F_{2}$, and $F_{3}$ ?. respectively.

The transfer functions to be determined from the secondary coolant flow oscillations are the response of $\Delta \mathrm{P} / \mathrm{P}_{0}, \Delta \mathrm{T}_{i}$, and $\Delta \mathrm{T}_{0}$ to the secondary fractional flow oscillation $\Delta \mathrm{V}_{2} / \mathrm{V}_{20}$. Let these transfer functions be denoted by $R_{1}, R_{2}$, and $R_{3}$, respectively.

The functions $F_{j}, G_{j}$, and $R_{j}(j=1,2,3)$ will be determined at 3 or 4 frequencies (e.g., Section 5.3.2). Their values at other frequencies can be estimated from a.plot of. the measured values.

In the first balanced oscillator test the inlet and outlet temperatures are to be held constant. Therefore, the following two complex 
equations must be satisfied.

$$
\begin{aligned}
& \Delta \mathrm{T}_{\mathrm{i}}=\mathrm{G}_{2} \Delta \mathrm{K}+\mathrm{F}_{2}\left(\Delta \mathrm{V}_{1} / \mathrm{V}_{10}\right)+\mathrm{R}_{2}\left(\Delta \mathrm{V}_{2} / \mathrm{V}_{20}\right)=0 \\
& \Delta \mathrm{T}_{\mathrm{o}}=\mathrm{G}_{3} \Delta \mathrm{K}+\mathrm{F}_{3}\left(\Delta \mathrm{V}_{1} / \mathrm{V}_{10}\right)+\mathrm{R}_{3}\left(\Delta \mathrm{V}_{2} / \mathrm{V}_{20}\right)=0
\end{aligned}
$$

Since the $G, F$, and $R$ functions are known, the relative phase angle and amplitudes of the reactivity and flow oscillations required to achieve a balance can be estimated from the above equations. There is not a unique solution to these equations, however", the solutions should be chosen so that the resultant amplitudes of the power oscillation (which can be estimated for a known reactivity oscillation by means of the curves shown in Section 5.4.4) and the flow oscillations are in the range of $\sim 2$ to $10 \%$ of the mean power level and flow rates. Analog simulation of the first balanced oscillator test ${ }^{(13)}$ indicates that such solutions are possible.

In the second balanced uscillator tcot the power level is to be held constant. Therefore, the following equation must be satisfied.

$$
\Delta P / P_{0}=G_{1} \Delta K+F_{1}\left(\Delta V_{1} / V_{10}\right)+F_{2}\left(\Delta V_{2} / V_{20}\right)=0
$$

Considerations similar to those for the first balanced oscillator test apply to the solutions of this equation. Specifically, the amplitude of the flow osclllations should be in the range of $\sim 2$ to $10 \%$ of the mean flow rate, and the coolant temperature oscillations should be $\lesssim \pm 15^{\circ} \mathrm{F}$. 
GEAP-5576

\section{$5.4 \quad$ BALANCED OSCILLATOR TESTS}

In the balanced oscillator tests the reactivity (poison rod) and the primary and secondary coolant flow rates are oscillated in a sinusoidal manner.

In the first balanced oscillator test the relative phase angles and amplitudes of the reactivity and flow oscillations are adjusted until the core inlet and outlet coolant temperatures remain constant. The flow and reactivity oscillations then remain unchanged as several cycles of data are recorded at the balanced condition.

In the second balanced oscillator test the relative phase angles and amplitudes of the reactivity and flow oscillations are adjusted until the reactor power (neutron flux) remains constant. The flow and reactivity oscillations then remain unchanged as several cycles of data are recorded at the balanced condition.

\subsubsection{Objective}

Successful performance and analysis of first and second balanced oscillator tests which are performed at the same mean reactor power, flow, and temperature conditions will provide values" for the fue $1^{\prime \prime}$ temperature coefficient, the fuel power coefficient, the coolantstructure temperature coefficient, the fuel "time constants, "and the reactivity-energy coefficient. By performing these tests at various power levels while maintaining a constant coolant temperature, and then performing the,tests at different coolant temperatures while maintaining a constant power level, the dependence of the reactivity 
coefficients and frequency response parameters. upon power and coolant temperature can be deţermined. . The power and coolant coefficients can be compared with the coefficients obtained from the static and conventional oscillator tests, and in addition, the frequency response parameters can be compared with the results obtained from the conventional osicillator teșts.

\subsubsection{General Procedure and Test Conditions}

A general procedure for all the oscillator tests is outlined in Section 5:0.2. This procedure can be used, where applicable, in the balanced oscillator tests.

Initial estimates of the "reactivity and" flow oscillations that are required for the balanced conditions will be àvailable from the flow and reactivity oscillatiouns described in Section $5: 3$ as well as from any previöus bălancéd ôscillätor tèsts. Minor adjustments in reactivity and flow will undoubtedly be required in order to öbtain a balanced condition. After initial transients have died out and the balance has

- beenn achievied, within the limits described bejow, the data.will be recorded.

$\therefore$ In the first balanced! oscillator tests the amplitude of the core inlet and outlet temperature oscillations should be held below ' while the amplitude of the power oscillation is: $\chi^{0.5} \mathrm{MW}$.

In the second balanced oscillator tests the amplitude of the power oscillation should be held below $\approx+0.2$ MW while the amplitude of the core coolant temperature oscillation $1 s \approx \pm 10$ to $15^{\circ} \mathrm{F}$. 
If the oscillations of the "balanced variable" is maintained within the above limits; the coolant temperature contribution to the reactivity feedback in the first balanced oscillator test will be " ¿10\% of the contribution caused by the power oscillation; and in the second balanced "oscillator test the contribution to the reactivity feedback that is caused by the power oscillation will be $\{10 \%$ of the contribution caused by the coolant temperature oscillation.

The signals of primary interest are:

1. The reactor power (neutron : flux) signal

2. The oscillator rod (reactivity) signal

3.... The inlet and outlet coolant temperature signals:

- from the instrumented fuel assemblies

4. : The primary and secondary coolant flow rates

5. The fuel temperature signals from the instrumented. ifuel assemblies

In addition, the absolute flow rates, power level, system temperature and reflector positions should be recorded.

The suggested test conditions for the balanced oscillator tests are shown in: Table 5-4.: Both a first and a second balanced oscillator test are to be performed at each of these reactor conditions $\therefore$. Low flow rates are recommended since this will decrease the effect of a non-zero temperature oscillation in the first balanced oscillator test (see the equation in Section $5 \cdot 4 \cdot 3 \cdot 2$ ). 
TABLE 5-4.

\section{BALANCED OSCILLATOR TESTS}

\begin{tabular}{ccc}
$\begin{array}{c}\text { Power } \\
\text { (MW) }\end{array}$ & $\begin{array}{c}\text { Flow } \\
(\%)\end{array}$ & \multicolumn{2}{c}{$\begin{array}{c}\text { Average Coolant Temperature } \\
(\text { ( } F)\end{array}$} \\
5 & 30 & 760 \\
10 & 40 & 560,760 \\
15 & 60 & 760 \\
19 & 75 & 760
\end{tabular}

A list of possible frequencies for the balanced oscillator tests are shown in Table 5-5... The tests can be performed using the frequencies 1isted under Case $A$ and then repeated (as a check) using the frequencies indicated by Case $B$. The poison rod is oscillated at $0.5 \mathrm{cps}$ in order to ensure proper normalization of the rod worth.

TABLE 5-5

POSSIBLE FREQUENCIES FOR BALANCED OSCILLATOR TESTS

Frequency

(cycles/sec)

$0.50(\operatorname{rod}$ only no balance)

0.025

0.016

0.010

0.0063

0.0040

0.0025

0.0016
Number of Cycles (after balance is achieved)

15

10

10

5

5

3

3

3

\section{Minutes for Specified Cycles}

Case A

Case B

1

1

7

10

9

9

13

13

13

20

32 


\subsubsection{Data Collection and Analysis}

The general procedures for data collection and analysis are described in Section 5.0.3, and these procedures can be used, where applicable, in the balanced oscillator tests..

As in the zero power and conventional oscillator tests (see Section 5.0.3 and 5.1.3.1) the dynamic measurement of the oscillator rod worth can be compared with the "static" measurement of the oscillator rod worth.

\subsubsection{First Balanced Oscillator Feedback}

The mathematical model for the feedback in the first balanced oscillator test is given in Section 2.5.4. In terms of this model, the condition equations can be written as follows:

$$
L(i \omega)=\left[h(i \omega)-\left\{C_{f} F_{s}(i \omega)+C_{q}\right\} U(i \omega)-C_{i} U_{i}(i \omega)\right]-\alpha_{f} F_{a v}(i \omega) x_{f}(i \omega)=0
$$

As before, this represents two equations: $\theta=\mathrm{REAL}(\mathrm{L})=0$ and $\phi=$ Imaginary $(\mathrm{L})=0$. These are the same condition equations as are used for the conventional oscillator test; however, In the first balanced oscillator test, the term $U(i \omega)$ and $U_{i}(i \omega)$ should be nearly zero and one seeks parameters $\alpha_{f}, \gamma$, and $\nu$ such that $\alpha_{f} F_{\text {av }}$ (iw) $x_{f}(i \omega)$ is equal to the "measured" term in the square brackets.

For the analyṣis. of this experiment, it is necessary to assume that $C_{f}, C_{q}, C_{i}$, and $F_{s}(i \omega)$, in addition to $h(i \omega), U(i \omega)$, and $U_{i}(i \omega)$ are known "measured" quantities. It is only when $U$ and $U$ are nearly zero (and their standard errors are small), that the values of $\alpha_{f}, \gamma$, and $\nu$, become independent of the value and standard errors assumed for $C_{f}, C_{q}, C_{i}$, and $F_{s}(i \omega)$. Since the value and standard error of $F_{s}$ depends on the values of $\gamma$ and $\nu$, it may be necessary to "re-estimate" $F_{s}$ after each iteration on $\gamma$ and $\nu$. 
In order to treat the term in the square brackets as a measured quantity, and to retain the procedure outlined in Section 5.4.3 and In Appendix $v$, define $x(j, n)$ for $j=1, \ldots 6$, as in Section 5.4.3. Redefine the parameters $\gamma$ and $\nu$, as they appear in $F_{s}$, not in $F_{a v}$, to be $n$ and $\rho,\left(i . e . F_{s}(i \omega, \gamma, v) \rightarrow F_{s}(i \omega, \eta, \rho)\right)$. Define additional "measured coordinates" $x(k, n)$ for $k=7,8, \ldots 11$, to be:

$$
\begin{array}{rlrl}
x(7, n) & =c_{f} & n & =1,2, \ldots . N \\
x(8, n) & =n & n & =1,2, \ldots . N \\
x(9, n) & =\rho & n & =1,2, \ldots . N \\
x(10, n) & =c_{q} & n & =1,2, \ldots . N \\
x(11, n) & =c_{i} & n & =1,2, \ldots . N
\end{array}
$$

Define the elements of the $\overline{\mathrm{P}}_{\mathrm{n}}$ matrix as in Section 5.2.3.

Let the elements $a(j)(j=1 ; 2,3)$ be defined by:

$$
\begin{aligned}
& a(1)=\alpha_{f} \\
& a(2)=\gamma \\
& a(3)=v
\end{aligned}
$$

The analysis from this point on is described in section 5.2.3.

\subsubsection{First Balanced Oscillator - Flow Measurements}

The mathematical model for the ratio of the relative flow oscillation to the relative power oscillation is given in Section 2.5.4. In terms of the model, the cundition equations can be writtcn no follows:

$$
\begin{aligned}
L(1 \omega) & =\frac{\Delta V(i \omega) / V_{0}}{\Delta P(i \omega) / P}-a_{c} x_{c}(i \omega)+\frac{V_{0}}{K_{n} V_{f}}\left\{\left(1+b-b F_{i}\right) U_{0}-\left(1-b F_{i}\right) U_{i}\right\} \\
& -\left(1-a_{c}\right) X_{f}(i \omega) F_{s}(i \omega)
\end{aligned}
$$




$$
\begin{aligned}
& \theta j=\operatorname{Rea} I^{\prime}(\mathrm{L})=0 . \\
& \phi_{i}^{\prime}=\text { Imaginary }(\mathrm{L})=0
\end{aligned}
$$

The quantities $a, X_{f}(i \omega), b(i \omega)$, and $F_{i}(i \omega)$ are calculated quantities: which are defined in Appendices II and III. The quantity: $K_{n}$ is the ratio of the steady-state core temperature rise at full. flow: $V_{f:}$ : to the total: power, $P$, and $v_{o}$, is the: mean flow rate.

Since the: procedure for: the analysis of the tests have been outlined in Appendix V;, and examples of the procedure have: been given. in previous: sections; only; the definition of the measured coordinates: and the parameters to be determined will be given here:

The measurediquantities are the 3 complex functions: $\left(\Delta \mathrm{V} / \mathrm{V}_{\mathrm{o}}\right) /\left((\Delta \mathrm{P} / \mathrm{P})_{0}\right)$ $\mathrm{U}_{0}$, and $\mathrm{U}_{i}$, and the real quantity $\mathrm{V}_{0}$. Thus, there are a total of 7 coordinates, the real: and imaginary; components of the complex functions and the: coordinates: $V_{0}$ : The coordinates: of the: complex functions:

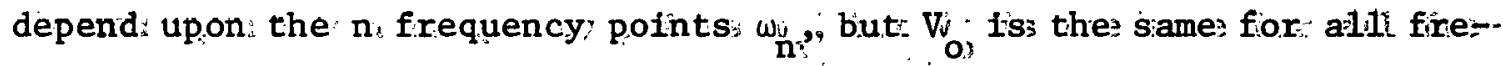
quencies: in ai given test:.. The standardi errorss in the: coordinatess of the complex functions,, likewise, depend upon the firequency $\omega_{n}$, but thes standard: errorc in $V_{0}^{i}$ is independent: of $n$..

\subsubsection{3i Second Ballancedi Oscililiator Tests:}

The condition equations for the analysis: of the second balanced oscillator test can be written (sees Section 2:5.5)) as follows:

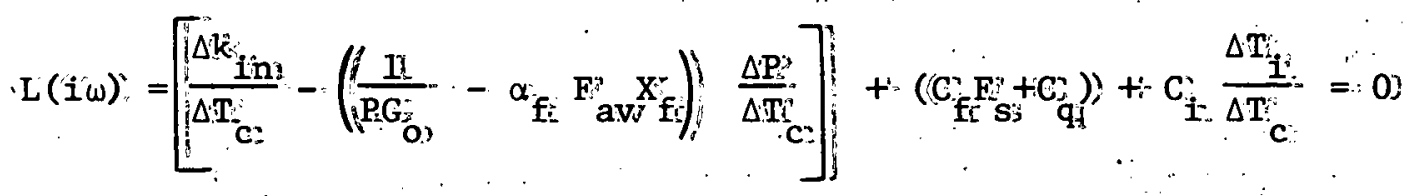


This represents two equations: $\theta_{.}=\operatorname{Real}(\mathrm{L})=0$, and $\phi=$ Imaginary $=0$.

In the analysis of the second balanced oscillator tests, one seeks parameters $C_{f}, \gamma, v, C_{q}$, and $C_{i}$ such that $\left(C_{f} F_{s}+C_{q}\right)+C_{i} \Delta T_{i} / \Delta T_{c}$ is the negative of the "measured" term in the square brackets above. Therefore, it is necessary, to assume that $\alpha_{f}$ and $F_{a v}$, in addition to $\Delta \mathrm{k}_{\mathrm{in}} / \Delta \mathrm{T}_{\mathrm{c}}, \mathrm{PG}, \Delta \mathrm{P} / \Delta \mathrm{T}_{\mathrm{c}}$, and $\Delta \mathrm{T}_{\mathrm{i}} / \Delta \mathrm{T}_{\mathrm{c}}$, are known "measured" quantities. It is only when $\Delta \mathrm{P} / \Delta \mathrm{T}$ c is nearly zero (i.e. when a near "balanced", condition is attained) that the value of $C_{f}, \gamma, \nu, C_{q}$, and $C_{i}$ become independent of the values and standard errors assumed for $\alpha_{f}$ and the parameters $\gamma$ and $\nu$ as they appear in $F_{a v}$.

The procedure for the analysis is discussed in Appendix $\mathrm{V}$ and examples for similar cases are outlined in sections 5.2.3 and.5.4.3.1. The quantities to be treated as measured variables and the quantities to be treated as unknown parameters have been defined above.

If su desired, the function $\mathrm{F}_{S}$, as determined from the analysis of the first balanced oscillator flow meașurements, may be treated as a "measured" function (note the comment at the end of Section 2.5.4). If this is the case, the parameters $\gamma$. and $v$ in $F_{s}$ are treated as measured coordinates rather than unknown parameters.

\subsubsection{Fuel Thermocouple Response}

The condition equation for the analysis of the response of the central fuel temperature to a power oscillation can be written (see Section 2.5.6) as:

$$
L(i \omega)=\frac{\Delta T_{c f}(i \omega)}{\Delta P(i \omega)}-K_{c} F_{c}(i \omega) X_{f}(i \omega)-G_{c}(i \omega) U(i \omega)=0
$$


The "measured" coordinates are the real and imaginary components of $\Delta \mathrm{T}_{\mathrm{cf}} / \Delta \mathrm{P}$, and $\mathrm{U}$. The parameters to be determined are $\mathrm{K}_{\mathrm{c}}$, and the two parameters in $F_{c}$ and $G_{c}, Y$ and $\nu$. The term $X_{f}$ is calculated as described in Appendix II. and $F_{c}$ and $G_{c}$ are described in Section 2.5.6.

The procedure and examples for the least squares fitting have been described in Section 5.2.3 and in Appendix V. All that remains is to define the partial derivations of $\mathrm{L}$ with respect to the two parameters $\gamma$ and $v$. These derivations are defined below:

$$
\begin{aligned}
& \frac{\partial L}{\partial \gamma}=-K_{c} X_{f} \frac{\partial F_{c}}{\partial \gamma}-U \frac{\partial G_{c}}{\partial \gamma} \\
& \frac{\partial L}{\partial v}=-K_{c} X_{f} \frac{\partial F_{c}}{\partial v}-U \frac{\partial G_{c}}{\partial v}
\end{aligned}
$$

The partial derivaties introduced above can be defined as follows:

$$
\begin{aligned}
& \frac{\partial F_{c}}{\partial \gamma}=\frac{F_{s} G_{c}-F_{c}}{\gamma+\nu^{2} / 4} \\
& \frac{\partial G_{c}}{\partial \gamma}=-i \omega F_{s} G_{c} \\
& \frac{\partial F_{c}}{\partial \nu}=\frac{\frac{2 \gamma}{\nu} G_{c}\left(G_{s}-F_{s}\right)-\frac{\nu}{2}\left(F_{c}-G_{c} F_{s}\right)}{i_{\gamma}+\nu^{2} / 4} . \\
& \frac{\partial G_{c}}{\partial \nu}=-i \omega \frac{\nu}{2} G_{c} F_{s}+2 i \omega \frac{\gamma}{\nu} G_{c}\left(F_{s}-G_{s}\right)
\end{aligned}
$$




\subsubsection{Expected' Results}

The calculated power-reactivity transfer functions for the first balanced oscillator tests are shown in Figures 5.4-1 through 5.4-6 for cases in which a perfect balance (zero coolant temperature oscillation) is attained. The power-reactivity transfer function for the first balanced oscillator tests is independent of the flow rate, provided a perfect balance is attained, however, the effect of non-zero power oscillations on the determination of $F_{S}$ is decreased with decreasing flow rate (see section 5.4.3.2) and the low flow rates of Table 5.4-1 are recommended.

The calculated reactivity-average coolant temperature transfer function for the second balanced oscillator tests are shown in Figures 5.4-7 and 5.4-8. Note that the value of the amplitude at very low frequencies is the sum of the fuel and coolant temperature coefficients, while the value of the amplitude at very high frequencies is the coolant coefficient only.

The effect of $a \pm 20 \%$ change in the Doppler coefficient on the first balanced oscillator tests is shown explicitly in Figures 5.4-1 to 5.4-6. The effect of a change in the Doppler or coolant coefficient in the results of the second balanced oscillator test (figures 5.4-7 and 5.4-8) is not shown explicitly. The effect of such changes can be readily visualized, however, since an increase in the coolant coefficient causes a constant increase in amplitude at low and high frequencies with little change in phase, while an increase in the Doppler coefficient causes an increase in the magnitude of the phase angle and an increase in the difference between the low and high frequency amplitude. 


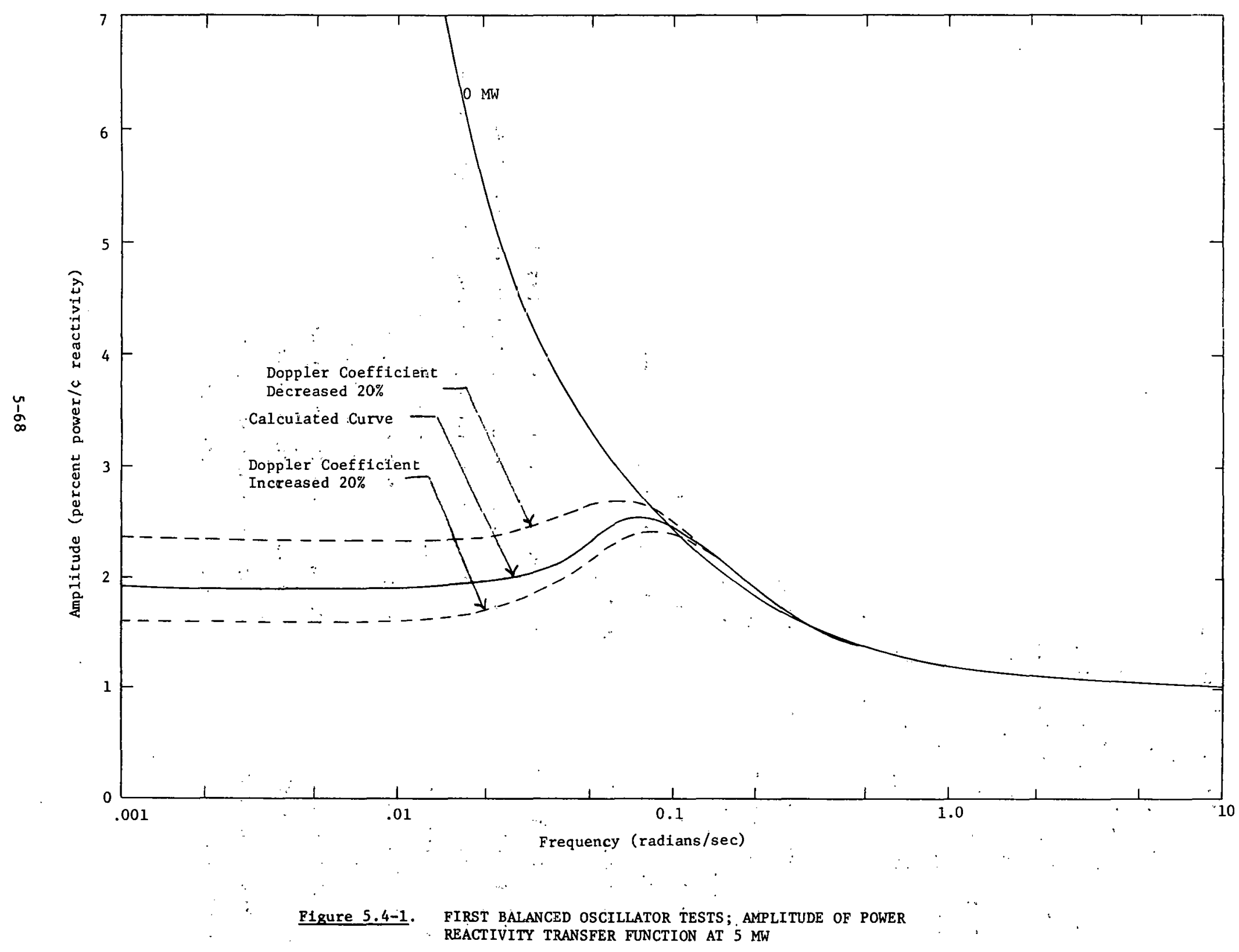




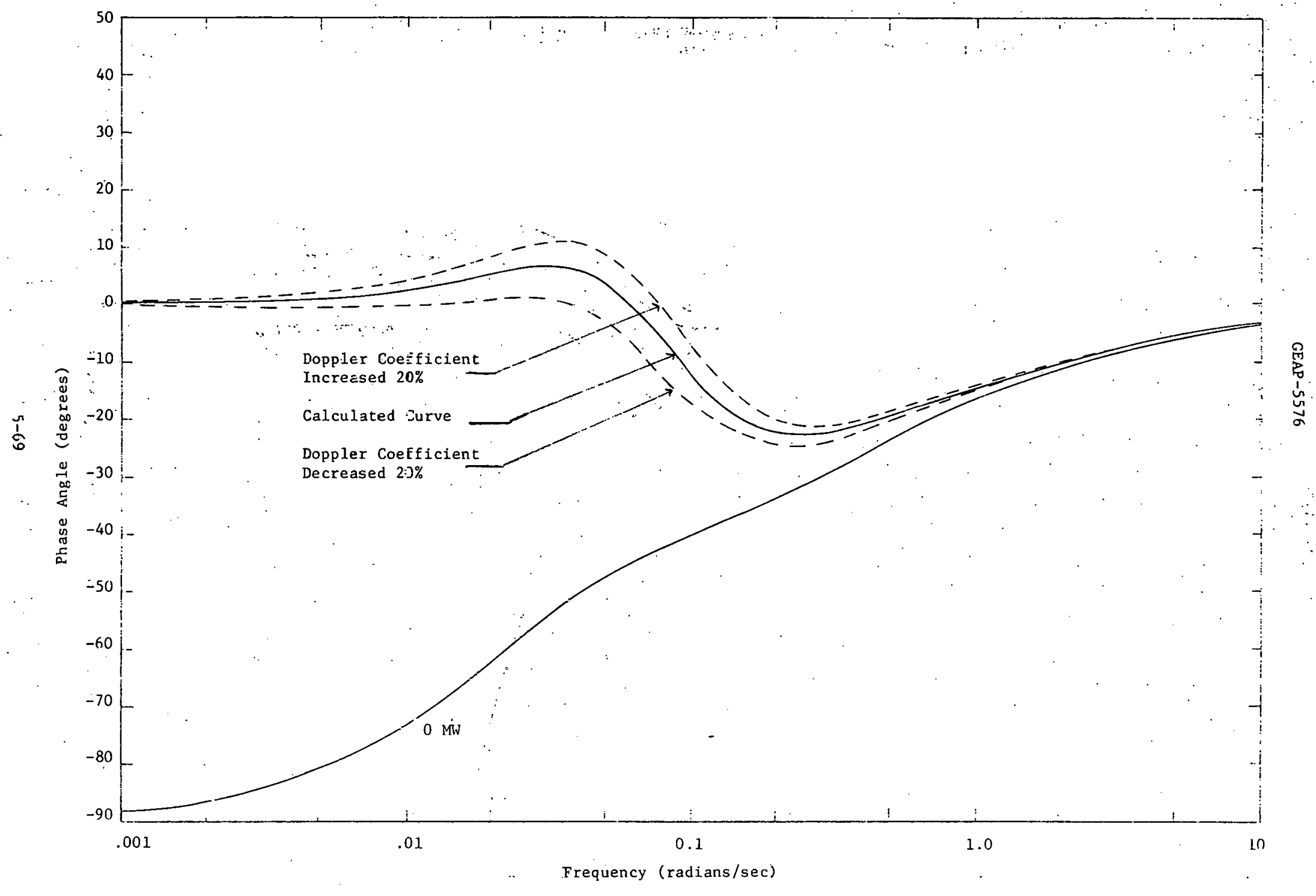

Figure 5.4-2. FIRST BALANCED OSCILLATOR TESTS; PHASE OF POWER REACTIVITY TRANSFER FUNCTION AT 5 MW 


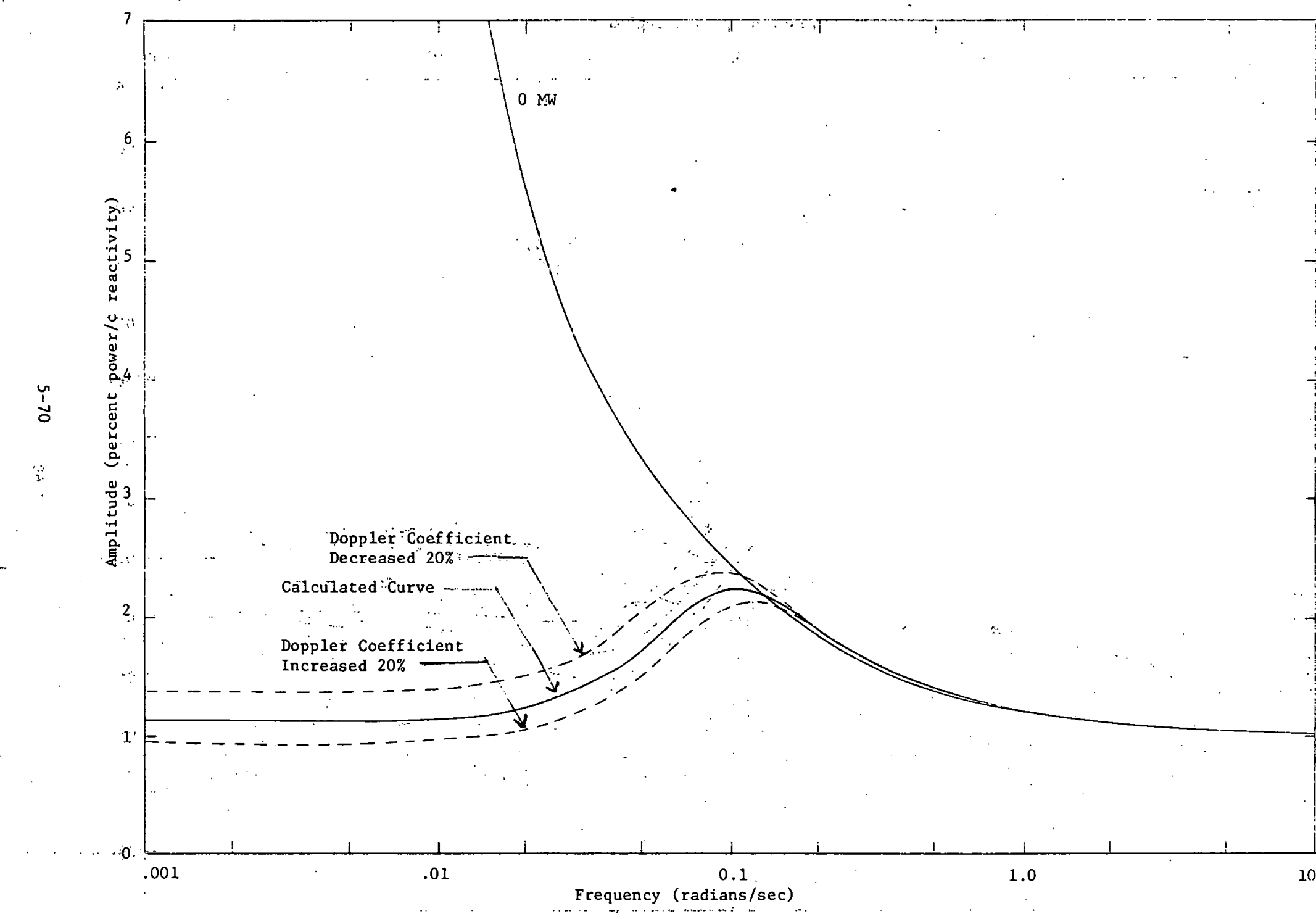

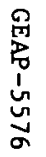

Figure 5.4-3: FIRST BALANCED OSCILLATOR TESTS; AMPLITUDE OF POWER REACTIVITY TRANSFER FUNCTION AT $10 \mathrm{MW}$ 


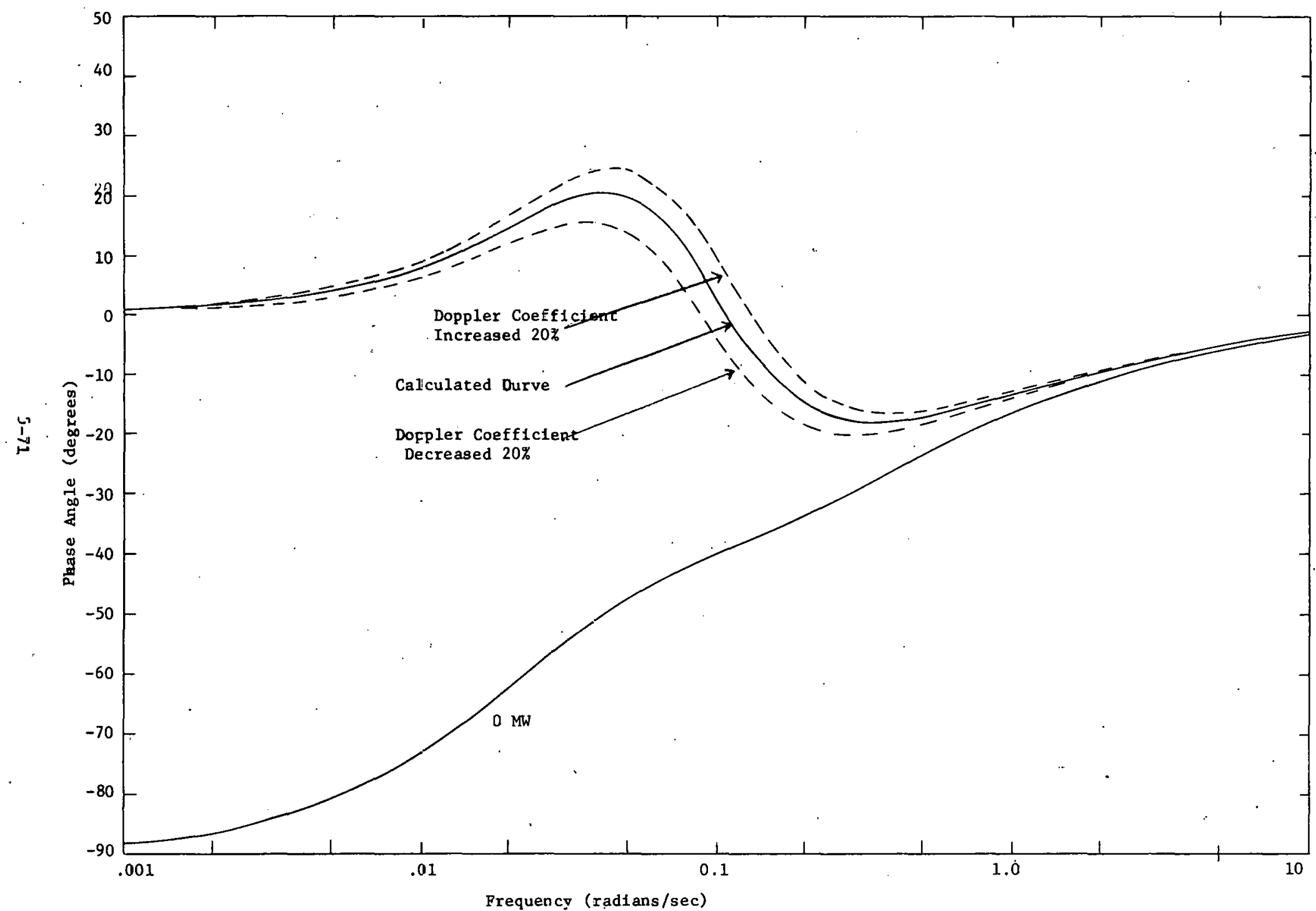

गั7

F1gure 5.4-4. FIRST BALANCED OSCILLATOR TESTS; PHASE ANGLE OF POWER REACTIVITY TRANSFER FUNCTION AT 10 MW 


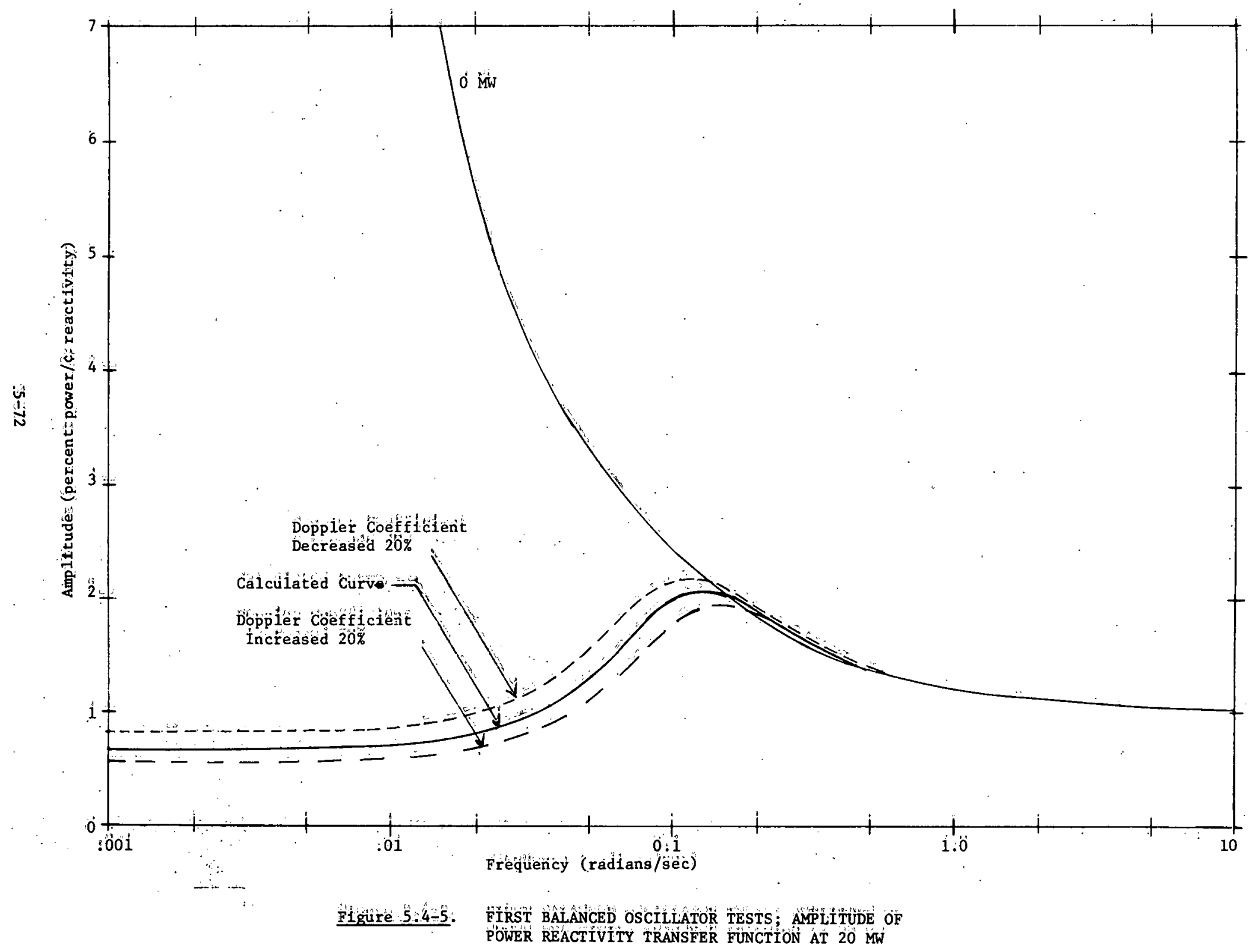




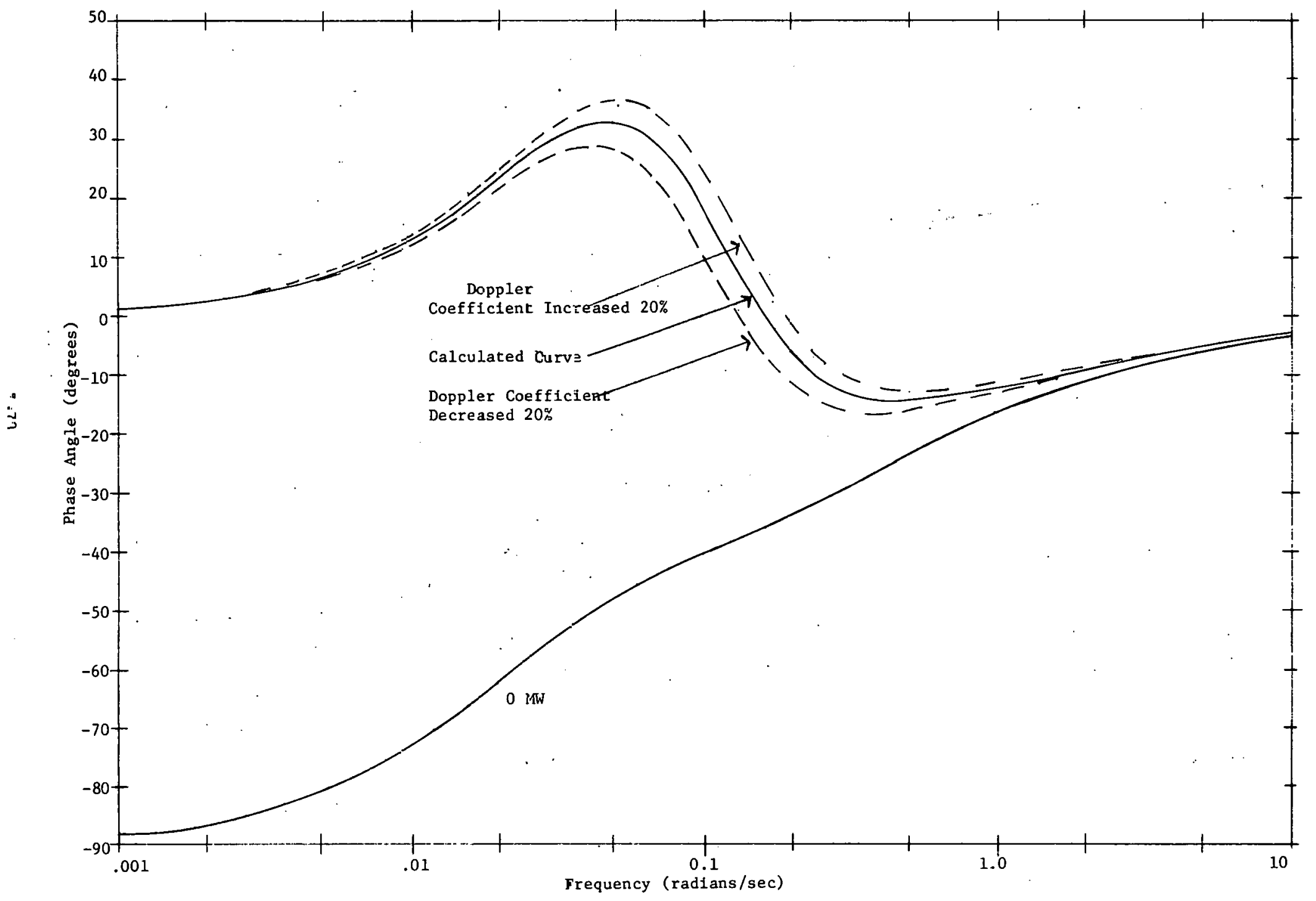

Figure 5.4-6. FIRST BALANCED OSCILLATOR TESTS; PHASE OF POWER REACTIVITY. TRANSFER FUNCTION AT $20 \mathrm{MW}$ 


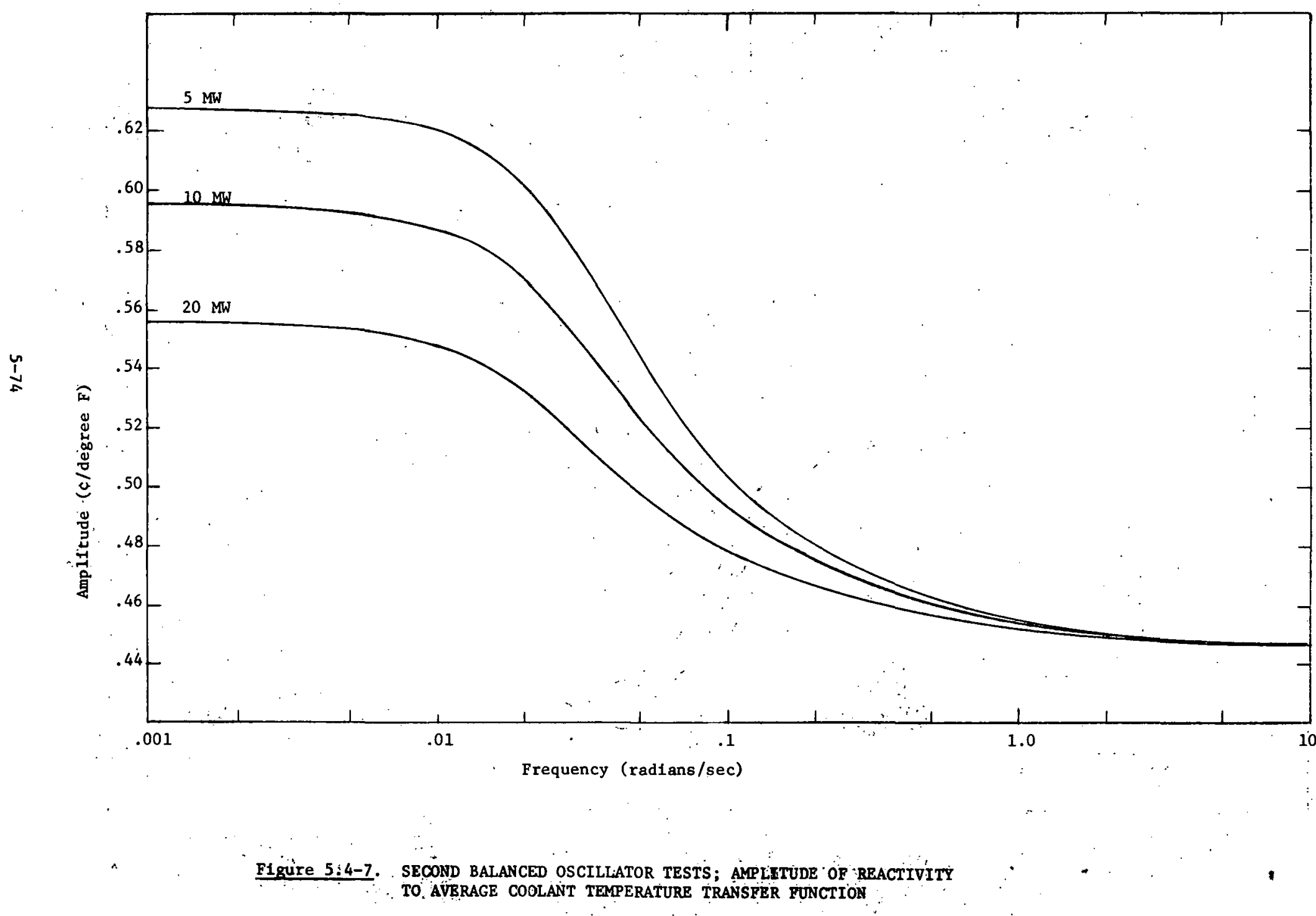




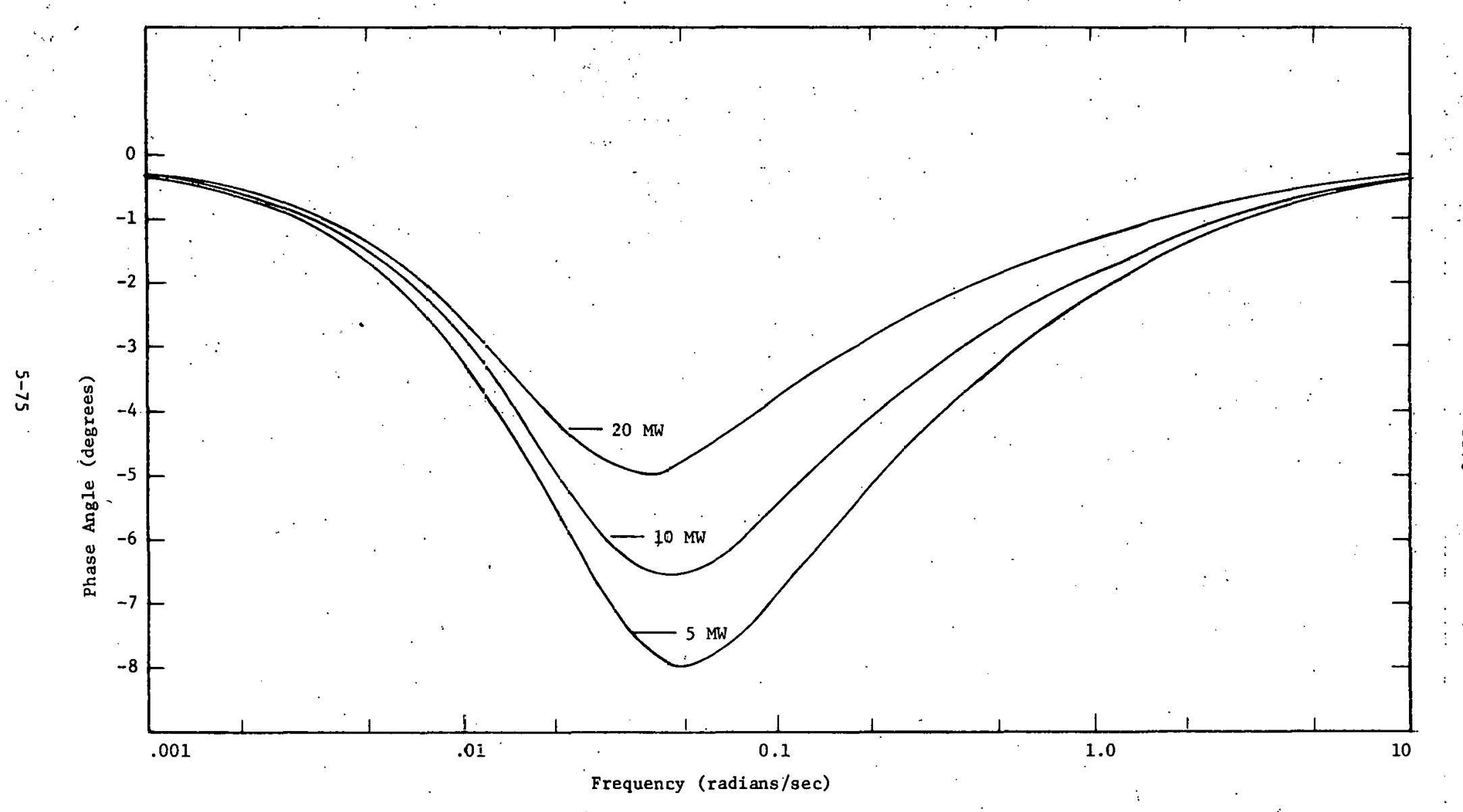

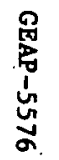

F1gure 5.4-8. SECOND BALANCED OSCILLATOR TESTS; AMRLITUDE OF REACTIVITY. TO AVERAGE COOLANT TEMPERATURE TRANSFER FUNCTION 
GEAP -5576

SECTION VI

TRANSIENT TESTS

\subsubsection{Objective}

The primary objectives of the transient tests are to demon- : ' strate the prompt Doppler, reactivity feedback and to determine the Doppler energy coefficient. In order to meet these objectives, both sub-prompt critical (positive reactivity insertion of less than one dollar) and super-prompt critical (positive reactivity insertion of greater than one dollar) tests will be performed. It is expected that sub-prompt critical transients will be performed at various power levels up to $\sim 15 \mathrm{MW}$ and that super-prompt critical tests will be performed at various power levels up to $\sim 10 \mathrm{MW}$ in order to determine the dependence of the Doppler energy coefficient upon the initial fuel temperature as well as the dependence upon the amount of energy released during a transient.

\section{0:2 Description of Tests}

In the transient tests, it may be necessary to experimentally verify, before each test or series of tests, that the desired poison slug is attached to the Fast Reactor Excurison Device (FRED) and that the available excess reactivity in the core (i.e. the remaining amount of reflector which can be raised) has not changed since a previous test. The worth of the FRED slug can be verified by the "rod swap" technique. The available excess reactivity can be determined, for example, by placing the reflectors in a prescribed configuration and comparing the resultant power level with a previous 
power level obtained for the same configuration; or by comparing reflector positions at a prescribed power level with previous positions for the same power level.

Once the FRED slug worth, available excess reactivity, and the operation of the FRED and the data acquisition system have been verified, the reactor is maintained at the specified test conditions of power, flow, and coolant temperature : for several minutes in order to ensure that static equilibrium is attained. The FRED is then fired while the data acquisition system is in the transient mode of operation. The reactor "will be "scramed" automatically a few hundred milliseconds after the initiation of the transient.

\subsubsection{Data Collection and Analysis}

The power (flux), fuel temperatures, and coolant temperature changes, as well as the time dependent FRED position, will be recorded by the data acquisition system. In addition, the following should be recorded in the log book:

1. Range setting on logarithmic amplifiers

2. Range setting on operational neutron monitors

3. Reflector positions

4. Poison slug worth

5. Main coolant venturi flowmeter

6. Pressure settings of the FRED

7. Reflector temperatures 
As in the oscillator tests, the transient analysis is based on the assumption that the "point reactor" kinetic equation is valid and that the delayed neutron parameters and $\ell / B$ (the ratio of the prompt neutron lifetime to the delayed neutron fraction) are known.

In order to determine' whether there are any changes in the spatial neutron flux shape during a transient, the ramp ejection of poison slugs worth $20.95 \$$ were simulated with the VARI-QUIR computer code. ${ }^{(1)}$ The results ${ }^{(2)}$ indicated that the maximum change in the spatial power shape during a transient is approximately $1 \%$ in a region immediately adjacent to the poison slug." Diring a simulated transient, the ratio of the high energy $(\geq 0.5 \mathrm{MeV})$ neutron flux to the average power level changed by $\delta 0.2 \%$ at a point corresponding to the U-238 fission chamber location in SEFOR. Any spatial changes in gamma flux at the SEFOR gamma detector 1ocation should be small, since the fraction of the gamma flux arising from thermal neutron capture is small. Thus, spatial effects should not invalidate the "point reactor" model.

A typiral analysis of the transient test data will include the. following steps:

1. The transient data, such as the logarithm of the power, the coolant temperature rise, and the fuel temperature rise is plotted in order to check the adequacy of the data. The plutting can bc performed by the data acquisition system. 
GEAP-5576

2. The total transient energy release is calculated and compared with predictions. The total energy release can be calculated in each of the following ways:

2a. The transient power data from the U-238 chambers can be combined with the data from the gamma chambers to provide a composite power trace. If the detectors are calibrated in the manner described in Section 2.2.2, the time integral of the power is the total excess energy which will eventually be produced (after the fission products produced by the excursion have decayed) in the core as a result of the transient. The actually energy: absorbed in the fuel, up to time $t$, using the numbers described in Appendix II, is $88 \%$ of this value.

2b. 'Although the fuel thermocouples in the instrumented fuel assemblies will not demonstrate the time dependent behavior of the fuel temperature, they should provide a measure of the maximum fuel temperature that results during the transient. This measurement can be used, along with the fuel heat capacity data, to calculate the energy released to the fuel.

2c. The coolant flow rate and the time dependent coolant temperature measurements from the instrumented fuel assemblies can also be used to obtain a measure of the total energy, produced. The peak coolant temperature will occur after the reactor has "scrammed": and the integration time of: the coolant temperature response must be long enough to allow the heat to diffuse from : the fuel. 
3. The transient power data is "smoothed" and used to calculate the net reactivity from the "point kinetic" equation. The SPORT computer program ${ }^{(3)}$ can be used for this purpose. SPORT also calculates the energy release (the integral of the power) as a function of time and provides a smoothed power trace.

4. The measured FRED position vs time and the measured (from the static tests) FRED worth vs position are used to calculate the time dependent FRED worth. This time dependent reactivity should agree, within experimental uncertainties, with the early portion of the net reactivity calculated by the SPORT program (particularly during the sub-prompt critical transients), since the feedback reactivity during the first portion of the transient is small. Calculated curves of the FRED reactivity as a function of time and of position are shown in Appendix. VII.

5. Calculate the time dependent feedback reactivity by subtracting the FRED reactivity from the net reactivity obtained in 3 above.

6. Calculate the non-Doppler feedback and subtract from the total feedback in order to obtain the Doppler Ieedback. The calculation of the non-Doppler feedback will be based upon calculated reactivity coefficients for the fuel and the fuel-clad axtal expansion effects and upon the measured coolant-structure reactivity coefficients. In order to use these reactivity coefficlents, the fuel, clad, coolant, and structure temperatures during the transients must also be calculated. The temperature 
changes can be estimated either by using the actual power trace in a code such as ARGUS (4), which calculates the various temperature changes, or by using the temperature changes (for the specific transient under consideration) which are predicted by a code such as FORE (5)

7. Using best estimates of the standard errors in the Doppler reactivity and the energy absorbed in the fuel, the Doppler energy coefficient is calculated using the least squares procedures outlined in Section 6.1 .3 and 6.2 .3 .

Although the uncertainties in the non-Doppler feedback may be large, calculations indicate that the Doppler effect during a transient is $\sim 80 \%$ of the total feedback, and the uncertainty in the Doppler energy coefficient is expected to be considerably less than the uncertalnties in the non-Doppler effects. Añ estimate of possible expected errors in the Doppler energy coefficient obtained from the analysis of transient tests is described in Appendix viII.

\subsection{SUB-PROMPT CRITICAL TRANSIENTS}

\subsubsection{Description of Tests}

The sub-prompt critical transient tests involve rapid insertion of less than $1.0 \$$ positive reactivity (i.e. rapid ejection of poison slugs worth $<1.0 \$$ ), in time intervals of the order of 100 msec. 
The initial fuel temperature rise is rapid so that heat conduction in the fuel is negligible (insofar as the fuel temperature is concerned) during the first 1 to 2 seconds (i.e. for times small compared to the $\sim 30 \mathrm{sec}$ fuel time constant).

The sub-prompt critical transient tests of primary interest are those in which the energy rcleased during the transient causes several cents of reactivity feedback. Such transients require initial power levels in the megawatt range and reactivity insertions of the order of 95c. Other transients, at lower initial power levels, and with smaller slug worths, will be run however, in order to check out the operation of the FRED, the transient power detector system, the data acquisition system, and the data analysis codes.

The sub-prompt critical transients of primary interest can result in peak power levels of 10 to 30 times the initial power and a maximum temperature rise in the average fuel of 100 to $200^{\circ} \mathrm{F}$ at rates up to $700^{\circ} \mathrm{F} / \mathrm{sec}$. At full coolont flow, the maximum clad and sodium temperature rises are approximately $20^{\circ} \mathrm{F}$ and $10^{\circ} \mathrm{F}$, respectively. The clad, structure, and sodium temperature rise during the first few tenths of a second are due primarily to direct gamma and neutron heating.

\subsubsection{Test Conditions}

For purposes of experimental program planning, it was assumed that the sub-prompt critical transient tests shown in Tables 6-1 and 6-2 will be performed. The actual number of tests which will be performed at each power level, and with each slug slze, will depend upon the results of the experiments; however, the tests shown in Tables 6-1 and 6-2 may provide an indication of the types of 
tests and total number of tests which will be performed.

The tests shown in Table 6-1 are performed primarily for the purpose of gaining familiarity with the system. The tests shown in Table 6-2 will provide information for the determination of the Doppler energy coefficient. These tests will provide energy coefficients, at three power levels between low and high power. The initial conditions for the tests correspond to reactor conditions at which oscillator tests are performed in order to facilitate comparison with the results of the oscillator tests.

TABLE 6-1

\section{SUB-PROMPT CRITICAL FAMILIARIZATION TESTS}

Power

(MW)

0.1

10
Slug Worth

(dollars)

0.40

0.70

0.85

$0.93 \rightarrow 0.98$

0.40

0.70

0.85
Number of Tests 2 2 2 3 2 2 2 
GEAY-5576́

TABLE 6-2

SUB-PROMPT CRITICAL TRANSIENTS

Power

(MW)

5

10

$\sim 15$
Slug Worth

(do1lars)

$0.93 \rightarrow 0.98$

$0.93 \rightarrow 0.98$

$0.93 \rightarrow 0.98$
Number of Tests

3

\subsubsection{Data Collection and Analysis}

The general data collection and analysis procedure for the sub-prompt critical tests are outlined in Section 6.0.3.

In the sub-prompt critical tests most of the reactivity feedback and energy release occurs after the FRED slug has been completely ejected. This effect can be utillzed in the following. manner. Lct the time of romplete FRED ejection be denoted by $t_{0}$ and let the FRED reactivity be denoted by $\Delta k_{F}(t)$. Then, for times $t>t_{0}$ the only change, $\delta k_{n}(t) \equiv \Delta k_{n}(t)-\Delta k_{n}\left(t_{0}\right)$ in the net reactivity, $\Delta \mathrm{k}_{\mathrm{n}} \equiv \Delta \mathrm{k}_{\mathrm{F}}+\Delta \mathrm{k}_{\mathrm{f}}$, will be due to changes. in the feedback reactivity $\Delta k_{f}$, because $\Delta k_{F}(t)=\Delta k_{F}\left(t_{0}\right)$ for $t>t_{0}$. Thus, analysis of $\delta k_{n}(t)$, rather than $\Delta k_{n}(t)$, eliminates the large error in $\Delta k_{f}\left(0 \leq\left|\wedge k_{f}\right| \leq 10 c\right)$ which could occur as a result of a small error in $\Delta k_{F}\left(0 \leq \Delta k_{f} \leq 95 \dot{c}\right)$. 
The model for the Doppler reactivity. feedback, $\Delta k_{D}(t)$, as a function of the energy absorbed in the fuel, $\Delta E_{f}(t)$, is given in Section 2.6 as:

$$
\Delta k_{D}(t)=n \Delta E_{f^{\prime}}(t)
$$

or as:

$$
\Delta k_{D}(t)-\Delta k_{D}\left(t_{o}\right)=n\left[\Delta E_{f}(t)-\Delta E_{f}\left(t_{o}\right)\right]
$$

where $t_{0}$ is the time of complete FRED ejection. The Doppler energy coefficient $n$ is a function of the initial power level (i.e. the initial fuel temperature) and may depend to some extent upon the energy release ( $1 . e$. a better representation for $\Delta \mathrm{k}_{\mathrm{D}}$ may be a quadratic in $\left.\Delta \mathrm{E}_{\mathrm{f}}\right)$.

In the above model, the "measured", (see discussion is 6.0.3, step no. 7); quartities are $\Delta k_{D}$ and $\Delta E_{f}$ and the parameter to be determined is $n$. "Let $k_{n}$ and $E_{n}$ denote the "measured" value of $\Delta \mathrm{k}_{\mathrm{D}}$ and $\Delta \mathrm{E}_{\mathrm{f}}$, respectively, at time $\mathrm{t}_{\mathrm{n}}$, and $1 \mathrm{et} \sigma_{\mathrm{kn}}$ and $\sigma_{\mathrm{En}}$ denote the standard errors in $k_{n}$ and $E_{n}$, respectively. If..equation (6.1-2) is used, let $k_{n}$ denote $\Delta k_{D}(t)-\Delta k_{D}\left(t_{j}\right)$ and let $E_{n}$ denote $\Delta E_{f}(t)$ $\Delta E_{f}\left(t_{o}\right)$. Then the Doppler energy coefficient $\eta$, is obtained from the following expression:

$$
\eta=\frac{1}{Q} \sum_{n=1}^{N} W_{n} E_{n} k_{n}
$$


where the weight, $W_{n}$, is defined by:

$$
\mathrm{w}_{\mathrm{n}} \equiv \frac{\sigma_{0}^{2}}{\sigma_{\mathrm{kn}}^{2}+\eta_{0}^{2} \sigma_{\mathrm{En}}^{2}}
$$

where $\sigma_{0}$ is an arbitrary constant; where $n_{0}$ is the initial estimate of $\mathrm{n}$; and where $Q$ is defined by:

$$
Q=\sum_{n=1}^{N} W_{n} E_{n}^{2}
$$

The sum, S, of the weighted squares of the residuals is given by:

$$
s=\sum_{n=1}^{N} W_{n}\left(k_{n}-\eta \dot{E}_{H}\right)^{2}
$$

and the estimated standard error, $\sigma_{\eta}$, in $\eta$ is given by:

$$
\sigma_{n}^{2}=\frac{s}{N-1}
$$

Once a value of $\eta$ is obtained from (6.1-3), this value can be used as the initial value $n_{0}$ for a new iteration. 
GEAP -5576

The overall uncertainty $\bar{\sigma}_{\eta}$ in $n$, which includes the effect of "biased errors" such as uncertainties in the absolute power level, and thus in the absolute energy release, can be estimated from the following expression:

$$
\frac{\bar{\sigma}_{n}^{2}}{n^{2}}=\frac{\sigma_{n}^{2}}{n^{2}}+\frac{\sigma_{\mathrm{P}}^{2}}{\mathrm{P}^{2}}
$$

where $\frac{\sigma_{\mathrm{P}}}{\mathrm{P}}$ is the fractional uncertainty in the absolute power, or energy, calibration.

\subsubsection{Expected Results}

The FORE ${ }^{(5)}$ computer code was used to calculate the reactor transient behavior, and the associated reactivity feedbacks, for several of the sub-prompt critical transients 1isted in Table 6.1-1 and 6.1-2. It was assumed in all cases that the FRED is completely ejected in $\sim 0.14$ seconds.

The calculated transient for two typical sub-prompt critical familiarization tests are shown in Figures 6.1-1 and 6.1-2. For comparison, the results of a $\$ 0.96$ transient at an initial power level of $0.1 \mathrm{MW}$ are shown in Figure 6.1-3 for a case in which the Doppler $\mathrm{T} \frac{\mathrm{dk}}{\mathrm{dT}}$ is only -0.004 and all other reactivity coefficients are zero. These curves show, as pointed out above, that the reactivity feedback is particularly small for transients beginning at low initial power levels. Thus, little or no information 
GEAP-5576

concerning the Doppler energy coefficient will be obtained from the tests listed in Table 6.1-1. However, these tests should provlde a means for gaining familiarity with the operating characteristics of the FRED.

Typical sub-prompt critical transients which will be used to obtain information concerning the Doppler energy coefficient are shown in Figures 6.1-4 through 6.1-6. For comparison, the transient behavior that would occur as a result of the same FRED ejection if the Doppler $\mathrm{T} \frac{\mathrm{dk}}{\mathrm{dT}}$ is only -0.004 and all other reactivity coefficients are zero, is shown in Figures 6.1-7 through 6.1-9.

As Figures 6.1-4 through 6.1-6 (or 6.1-7 through 6.1-9) indicate, most of the reactivity feedback occurs after the FRED is completely ejected (see Section 6.1.3 for the significance of this effect upon the determination of the reactlvity feedback). 
GEAP-5576
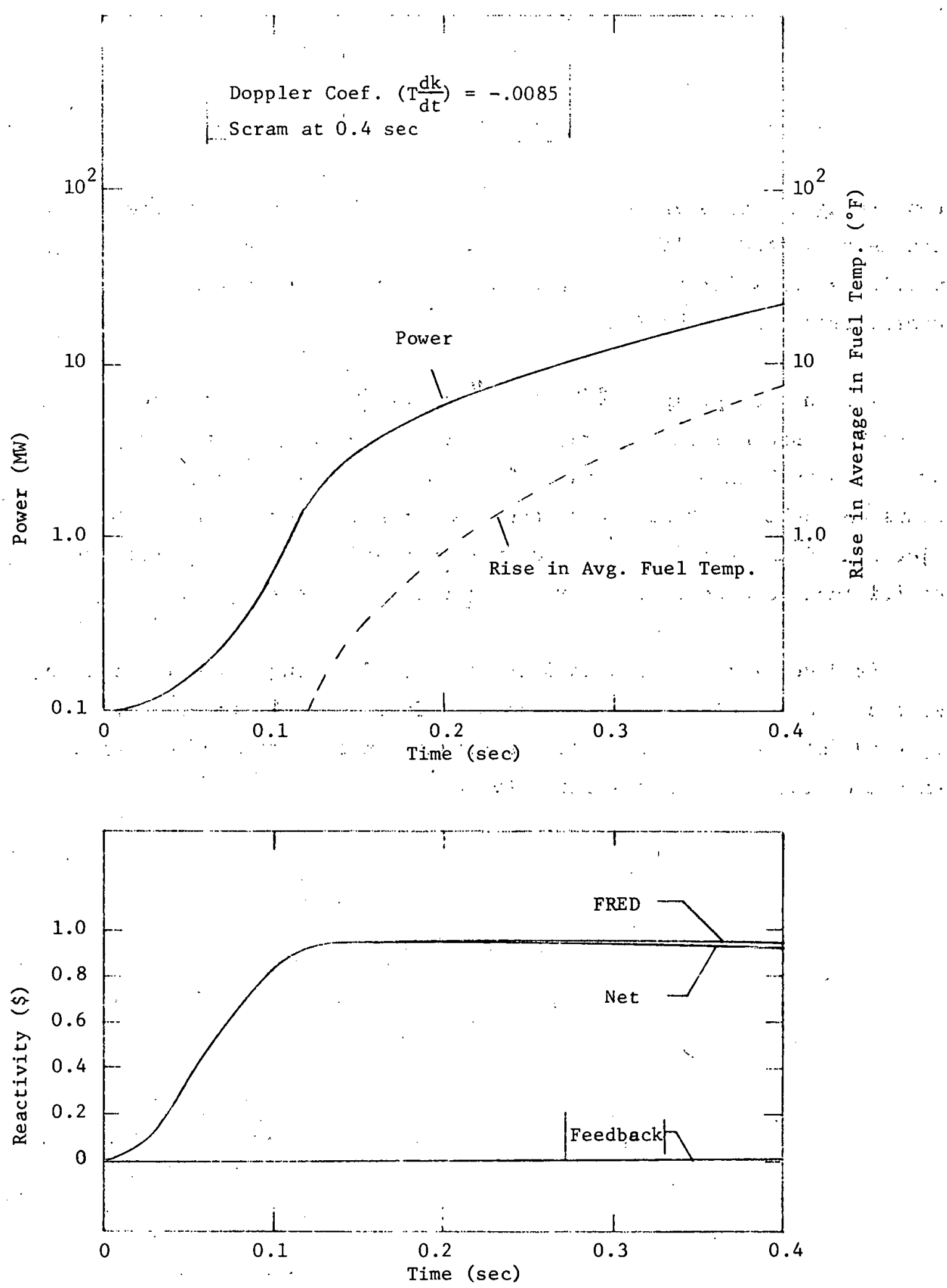

Figure 6.1-1 \$0.96 TRANSIENT AT 0.1 MW FOR CALCULATED COEFFICIENTS 

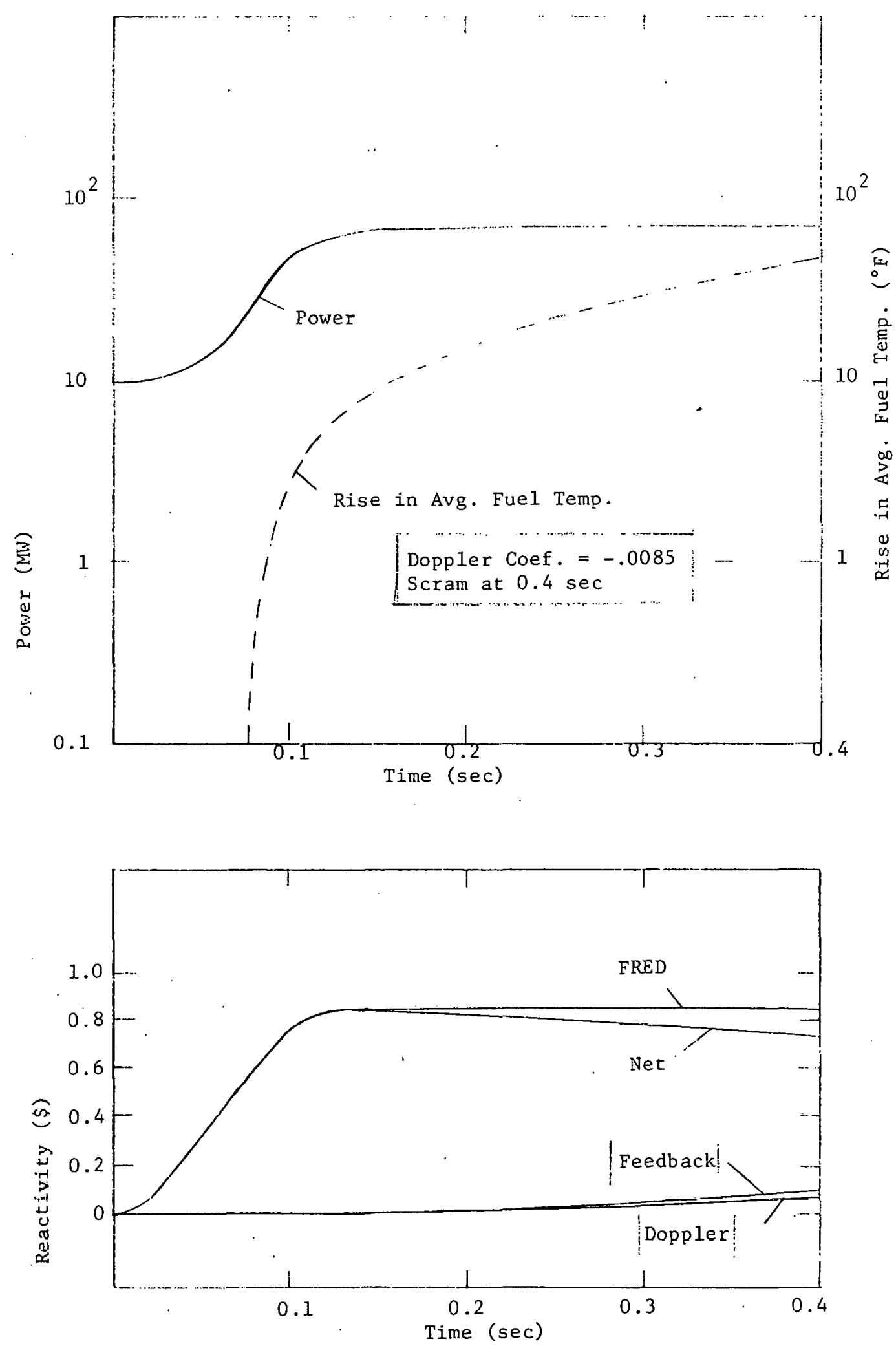

Figure 6.1-2. \$0.85 TRANSIENT AT 10 MW FOR CALCULATED COEFFICIENTS 

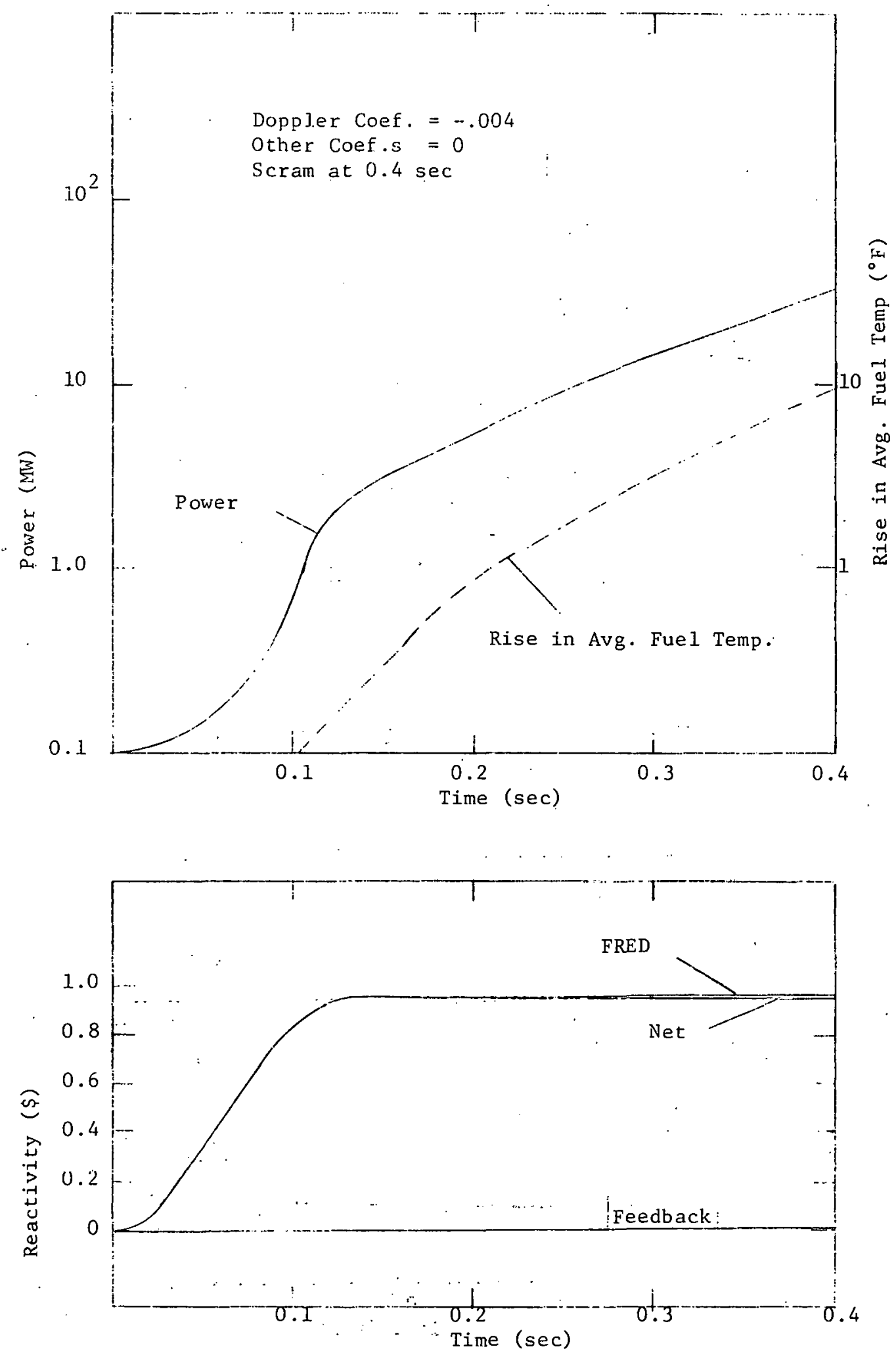

Figure 6.1-3 \$0.96 TRANSIENT AT 0.1 MW FOR -.004 DOPPLER 

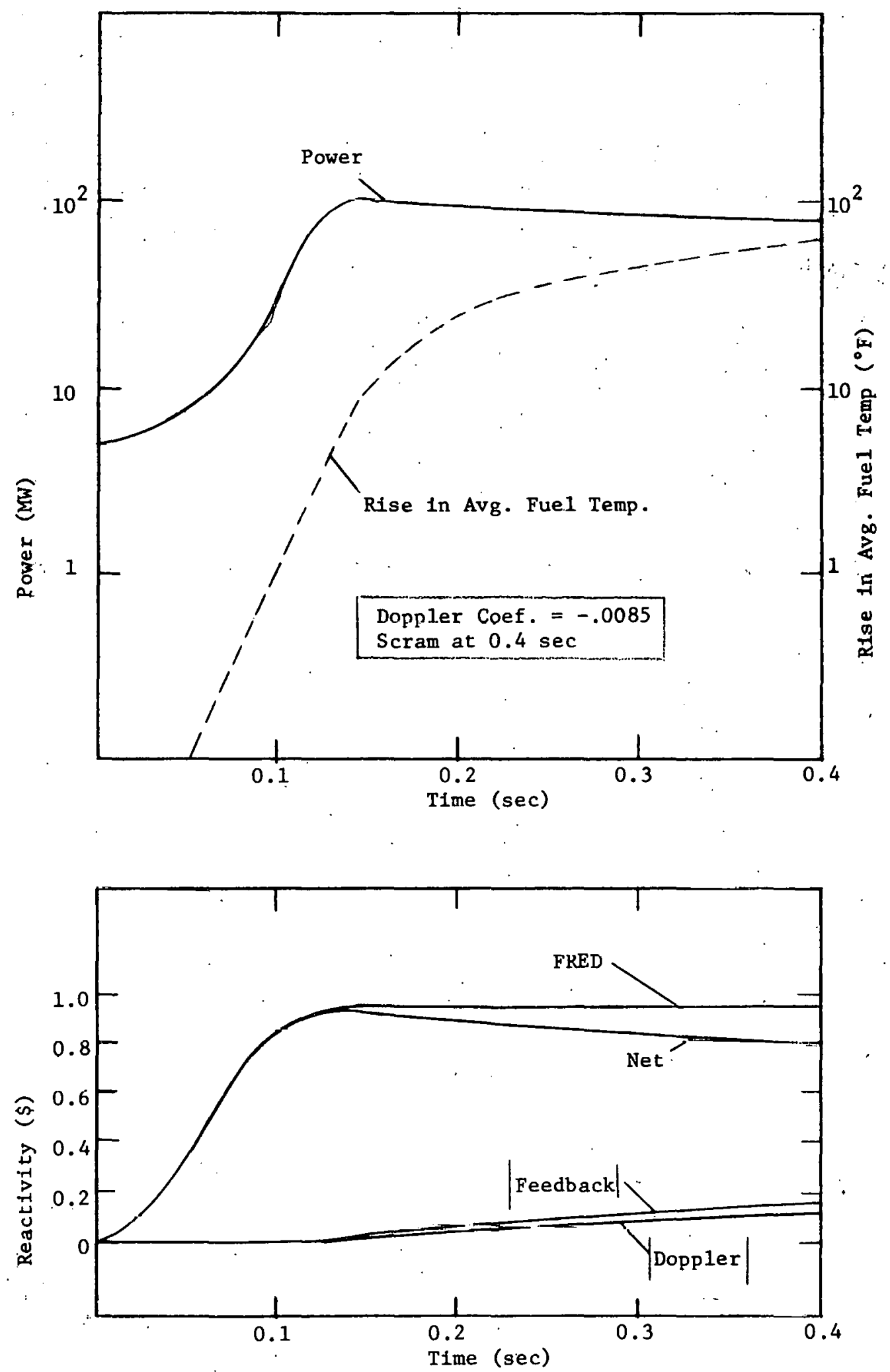

' Figure 6.1-4 \$0.96 TRANSIENT AT 5 MW FOR CALCULATED COEFFICIENTS 

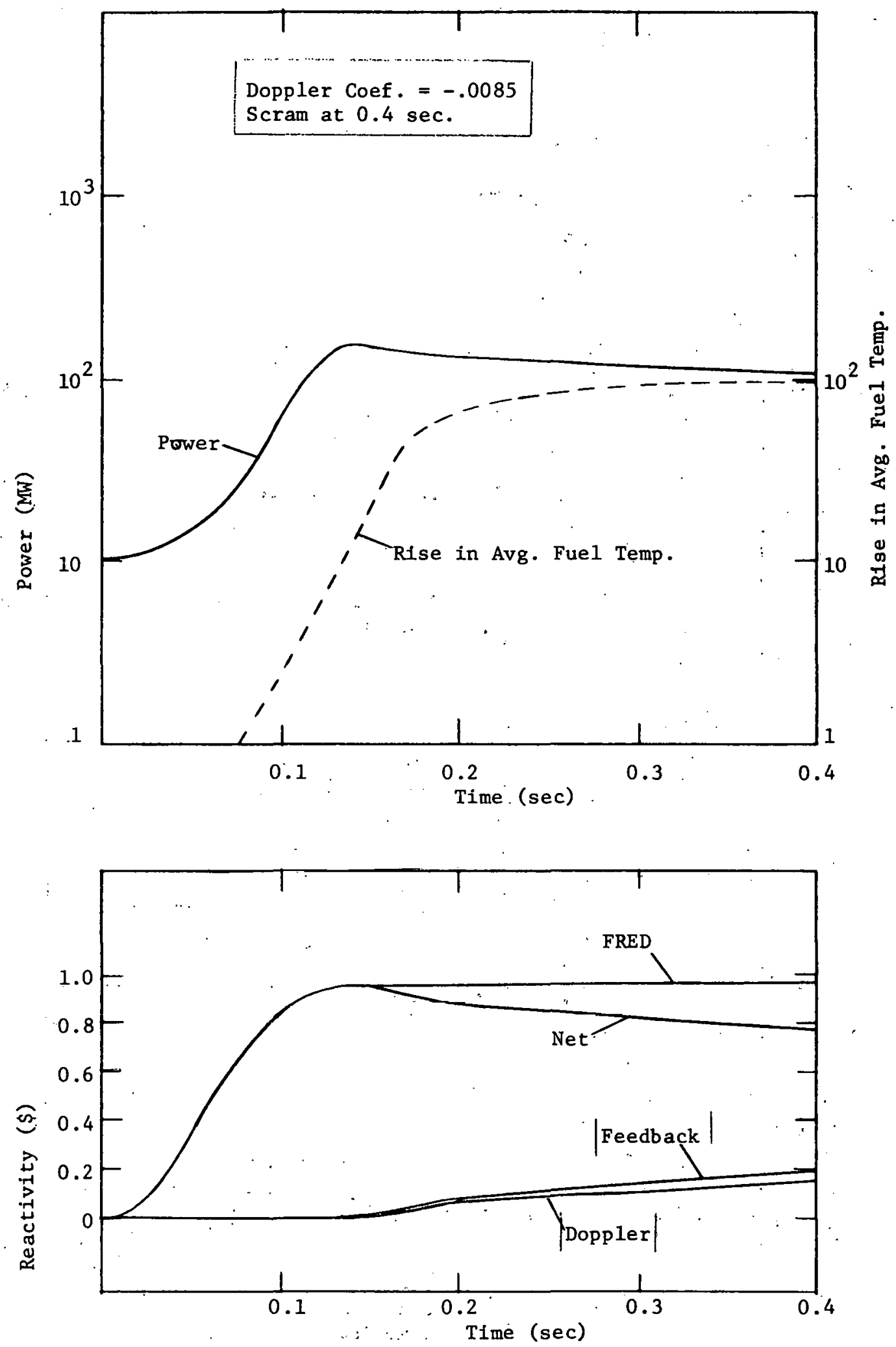

Figure 6:1-5 \$0:96. TRANSTENT AT $10^{\circ}$ MW FOR CALCULATED COEFFICIENTS 

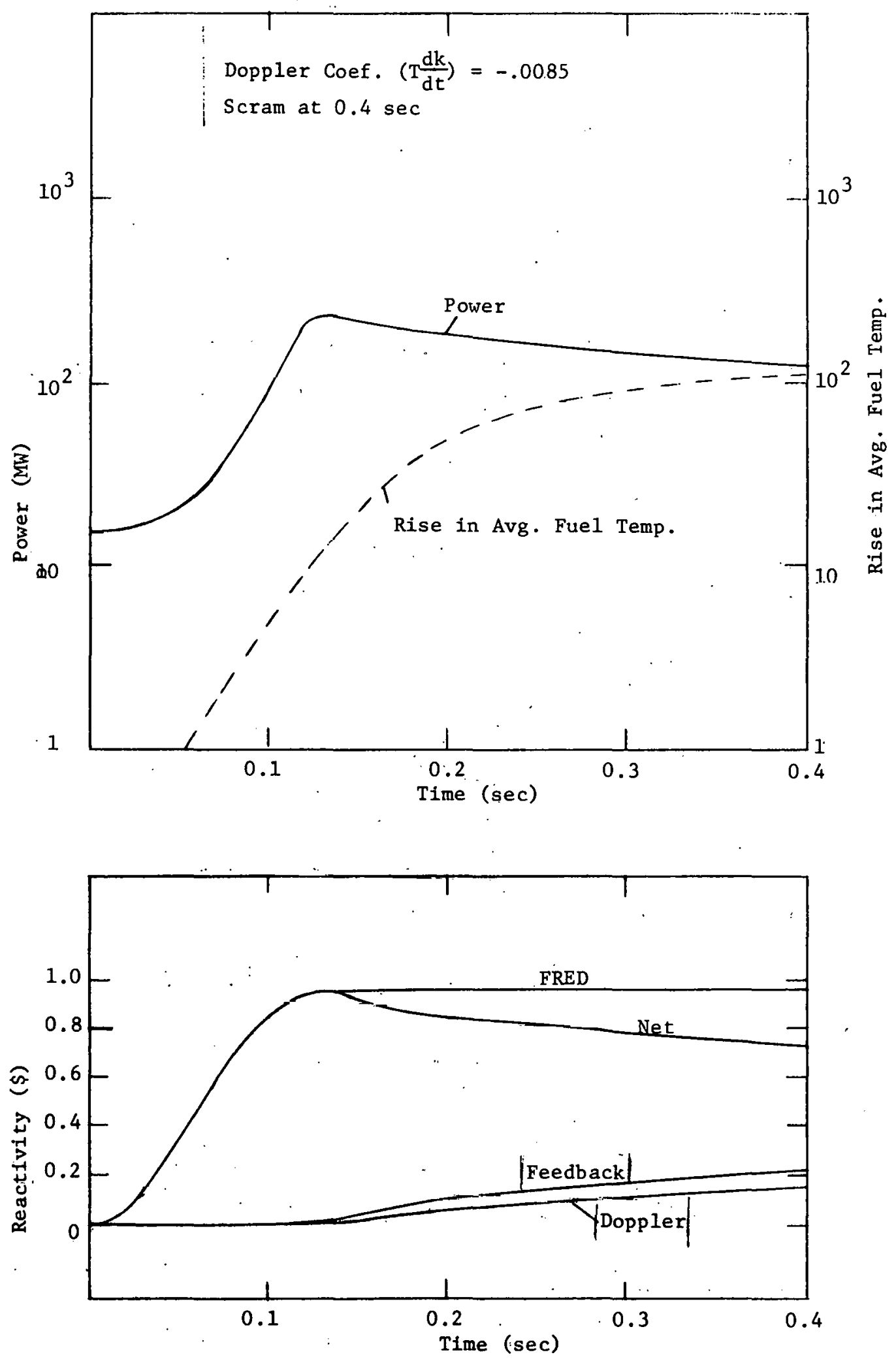

Figure 6.1-6 \$0.96 TRANSJENT AT 15 MW FOR CALCULATED COEFFICIENTS 

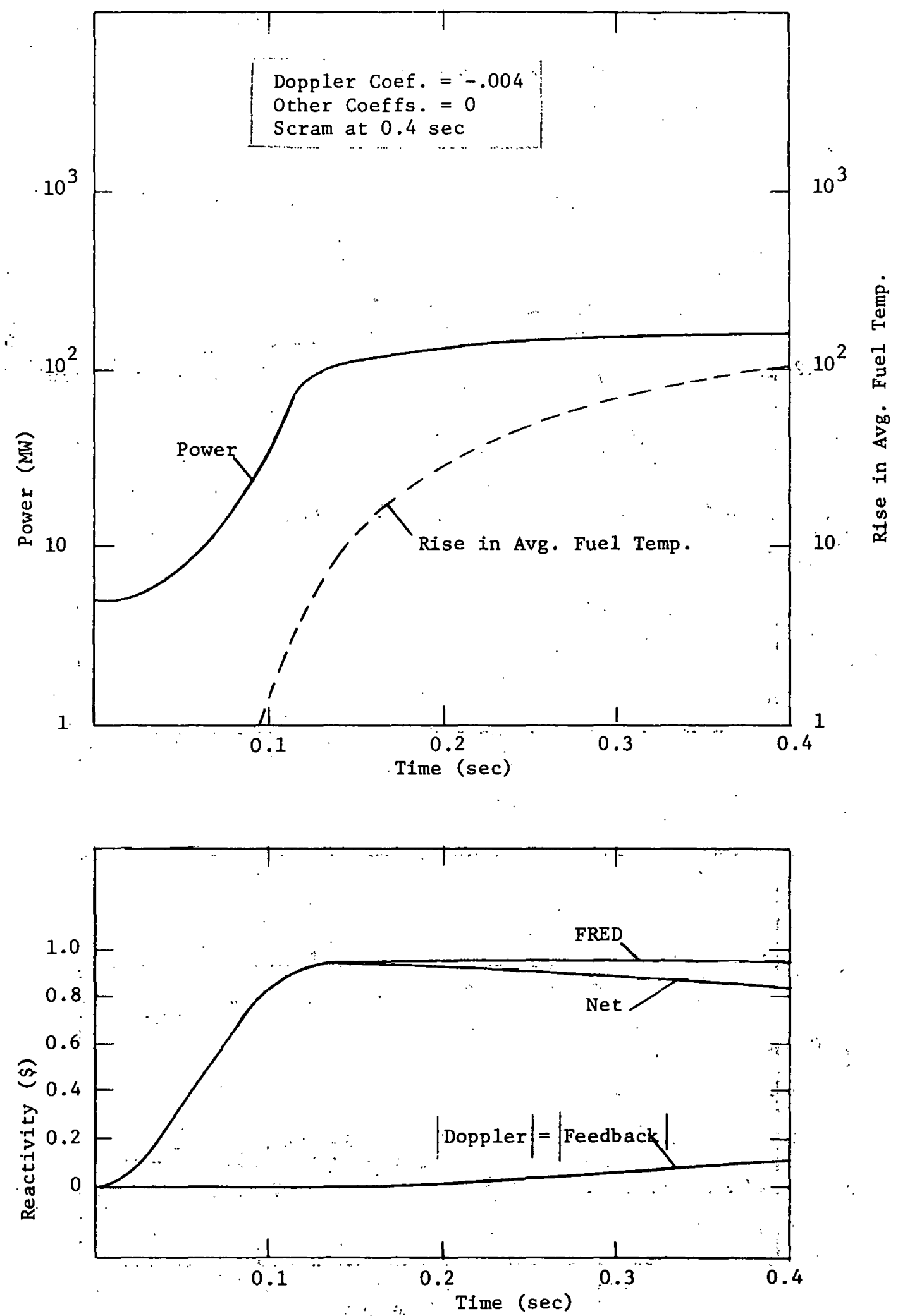

Figure 6.1-7 $: \$ 0.96$. TRANSIENT. AT 5,MW FOR -.004 DOPPLER 

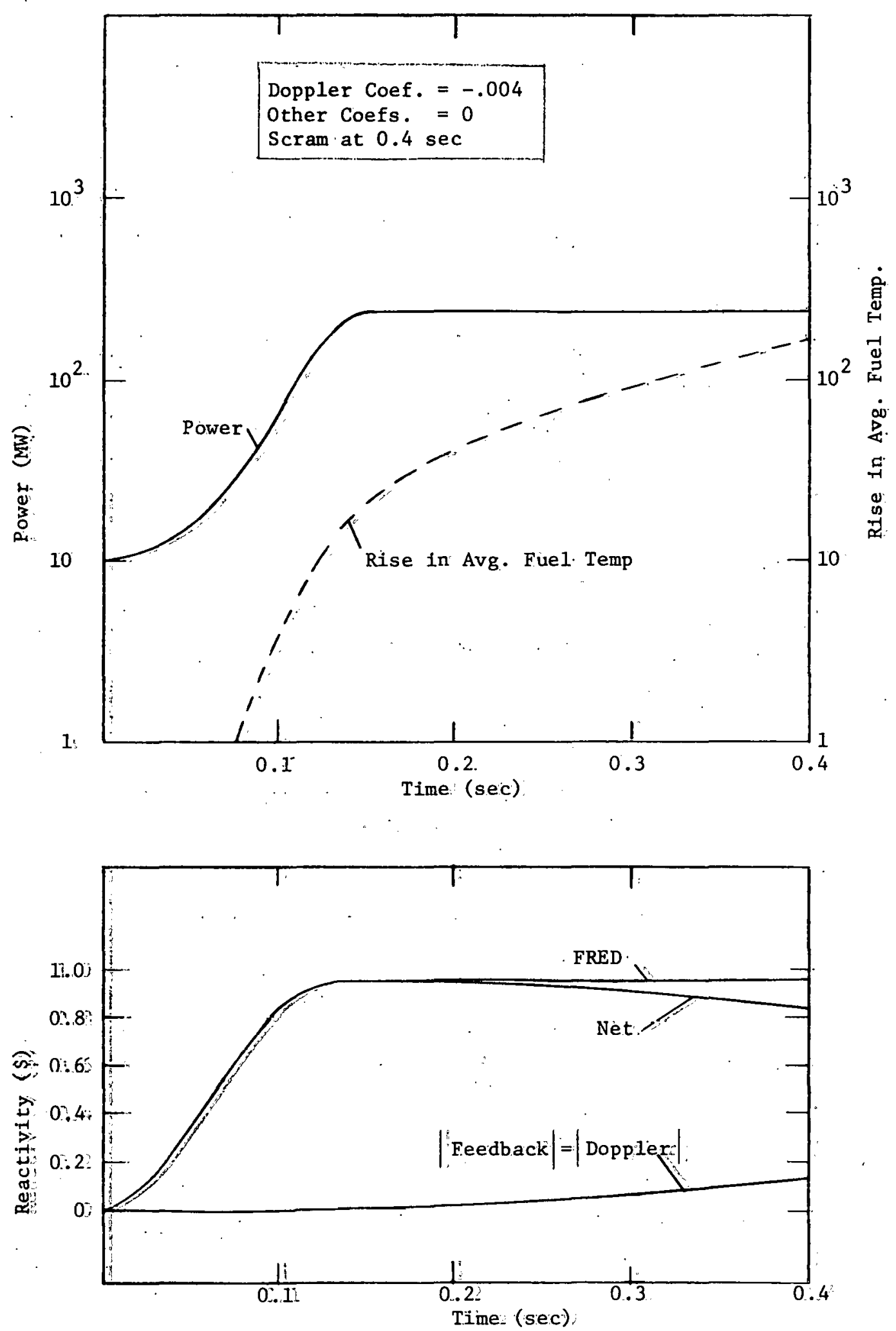

FIgurea $65.1-83$ \$0:.965 TRANSIENT: AT 10 MW FOR -.004 DOPPLER 

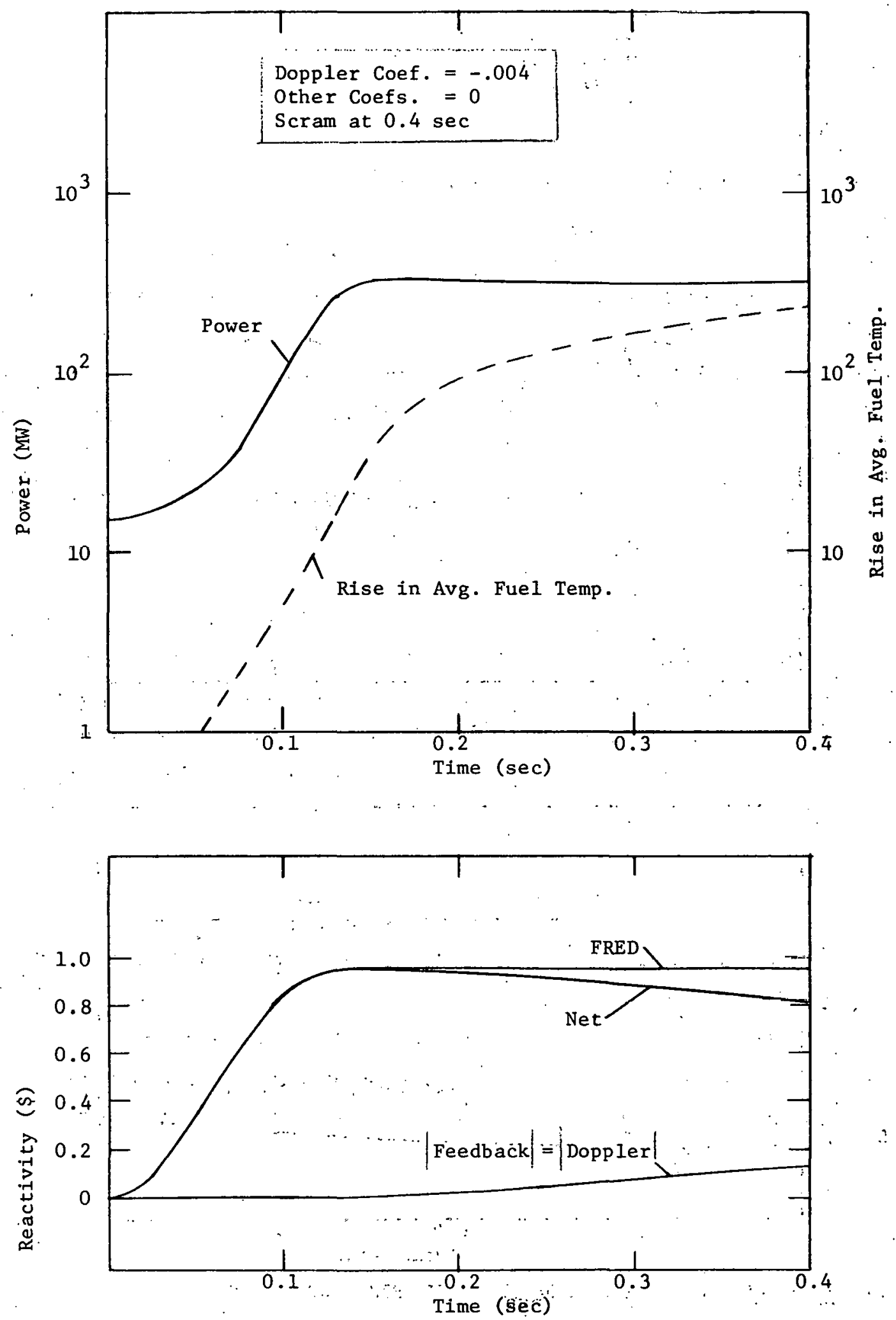

Figure 6.1-9 \$0.96 TRANSIENT AT 15 MW FOR -.004 DOPPLER 
GEAP-5576

\subsection{SUPER-PROMPT CRITICAL TRANS̈IENTS \\ 6.2.1. Description of Tests}

The super-prompt critical tests involve rapid insertion of more than $1.0 \$$ positive reactivity. Maximum planned transients of this type will result in peak power levels of $10^{4}$ to $10^{5}$ times the initial power level and a maximum temperature rise in the average fuel of $\sim 400^{\circ} \mathrm{F}$ at maximum rates of $\sim 10,000^{\circ} \mathrm{F} / \mathrm{sec}$. The reactivity effects due to clad,.. structure,... and sodium temperature rise may be 15 to $25 \%$ of the fuel reactivity effect.

Tests using reactivity insertions in the range of $1.0 \$$ to $1.3 \$$ in approximately 0.1 second are of primary interest, since these result in significant fuel temperature rise independent of heat conduction effects. An error analysis (see Appendix VIII) Indicates that 1llLle increase in accuracy can be obtainad by using reactivity insertions $>1.3 \$$.

\subsubsection{Test Conditions}

For the super-prompt critical transients, two different sers of lest conditions are listed (Tables 6-3 and 6-4). The set that will be used will depend upon the Doppler coefficient that is determined from the static and oscillator tests. The actual number of tests shown in Tables $6-3$ and $6-4$ are listed for program planning purposes. 
Either sets of tests (Table 6-3 or Table 6-4) will serve to demonstrate the ability of the Doppler effect to turn around a prompt critical excursion under temperature conditions approaching those of a large power reactor. Since static; osciliator; and sub-prompt critical tests will have already been performed at the initial power and temperature conditions of these tests, a direct comparison of results is afforded.

TABLE $6 \div 3$

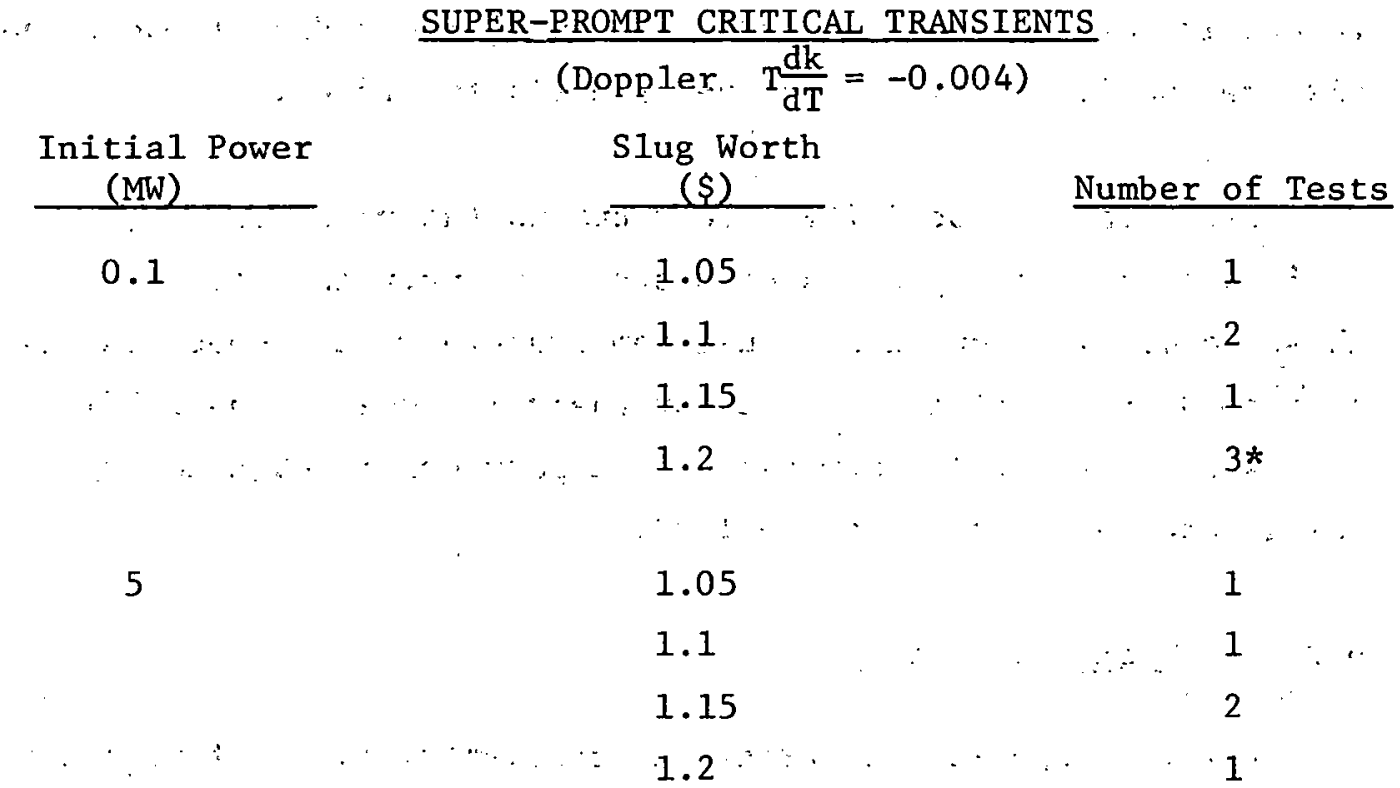

* Repeát and rate change 
GEAP -5576

TABLE 6-4

SUPER-PROMPT CRITICAL TRANSIENTS

(Doppler $\mathrm{T} \frac{\mathrm{dk}}{\mathrm{dT}}=.0 .008$ )

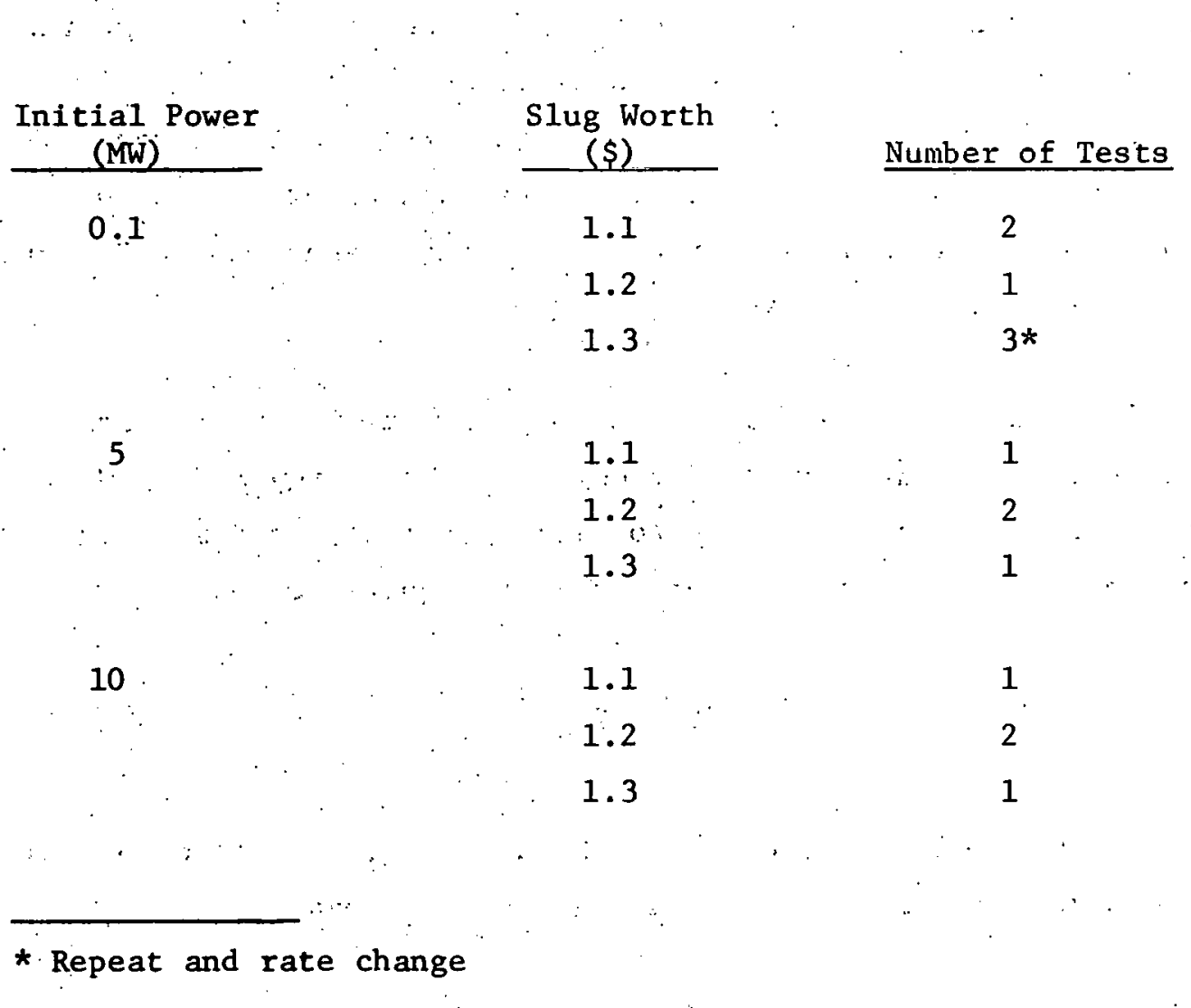




\subsubsection{Data Collection and Analysis}

The general data collection and analysis procedures for the super-prompt critical tests are outlined in Section 6.0.3, and the analysis is similar to the analysis of the sub-prompt critical transients. Unlike the sub-prompt critical transients, however, a significant portion of the reactivity feedback occurs while the poison FRED slug is being ejected ạnd the time dependent FRED worth needs to be accurately known.

Calculations indicate that the Doppler reactivity feedback can be well represented by a polynomial in the energy absorbed in the fuel. The model for the Doppler reactivity is discussed in Section 2.6.1 and is reproduced here for convenience.

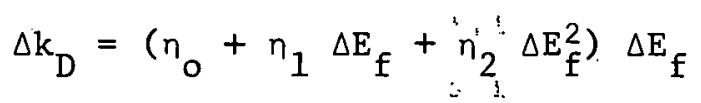

The "measured" quant1ties are $\Delta \mathrm{k}_{\mathrm{D}}$ and $\Delta \mathrm{E}_{\mathrm{f}}$ (see Section 6.0.3) and the parameters to be determined are $n_{0}, n_{1}$, and $n_{2}$

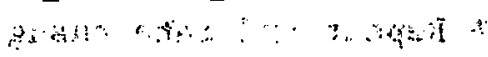

Let $k_{n}$ and $E_{n}$ denote the "measured" values of $\Delta k_{D}$ and $\Delta E_{f}$, respectively, at time, $t_{n}$, and let $\sigma_{k n}$ and $\sigma_{E n}$ denote the standard errors in $k_{n}$ and $E_{n}$, respectively. In addition, using the notation of Appendix $V$, let the elements, $a_{j}(j=1,2,3)$ of a column vector, $\underset{\sim}{A}$, correspond to the parameters $n_{j}(j=0,1,2)$ and let the vector, $A_{\sim}$, denote the initial estimate of the vector $\mathrm{A}$. 
For the above model, equation (24) of Appendix $V$ reduces to:

$$
T_{j k}=-\sum_{n=1}^{N} \frac{\sigma_{0}^{2}}{\sigma_{n}^{2}} E_{n}^{j+k} .
$$

where (see equations (9) and (10) of Appendix V), for uncorrelated errors in $k_{n}$ and $E_{n}$, $\sigma_{\eta}^{2}$ in the above equation 1 defined by:

$$
\sigma_{n}^{2}=\sigma_{k n}^{2}+\left[a_{10}+2 a_{20} E_{n}+3 a_{30} E_{n}^{2}\right]^{2} \sigma_{E n}^{2}
$$

Since the "condition equation" is linear in the unknown parameters $a_{j}$, the equation for the estimated values of the $a_{j}$ can be written as:

$$
\stackrel{A}{A}=\bar{Q} \underline{\sim}^{l}
$$

where $\bar{Q}=(\bar{T})^{-1}$ and where the elements $C_{j}^{l}$ of $\underset{\sim}{C^{l}}$ are defined by:

$$
c_{j}^{l}=\sum_{n=1}^{N} \cdot \frac{\sigma^{2}}{\sigma_{n}^{2}} k_{n} E_{n}^{j}
$$

The value of the sum, $S$, of the, weighted squares of the residuals is given. by:

$$
s=\sum_{n=1}^{N} \frac{\sigma_{0}^{2}}{\sigma_{n}^{2}}\left[k_{n}-\sum_{j=1}^{3} a_{j} E_{n}^{j}\right]^{2}
$$

where the $a_{j}$ are obtained from $(6,2-1)$. 
The estimated standard error $\sigma_{j}$ in $a_{j}$ is given by:

$$
\sigma_{j}^{2}=Q_{j j} \frac{S}{N-3}
$$

and the confidence bands for the reactivity feedback can be obtained by the procedure described in Appendix. $V$.

\subsubsection{Expected Results}

The time dependence of the power, the rise in average fuel temperature, and of the reactivity feedback are shown in Figures 6.2-1 through 6.2-6 for the $\$ 1.1$ and $\$ 1.3$ transients listed in Table 6.2-2.

For comparison, the effect of an -0.004 Doppler $\mathrm{T} \frac{\mathrm{dk}}{\mathrm{dT}}$, with other reactivity feedback coefficients set to zero, on the $\$ 1.3$ transients is shown in Figures 6.2-7 to 6.2-9. The latter three transients are not a part of the experimental program. (see Table 6.2-1 for the transients that are planned for the case of .. an -0.004 Doppler $T \frac{\mathrm{dk}}{\mathrm{dT}}$ ) and the results are included solely for the purpose of demonstrating the effect of decreased reactivity feedback.

As the figures indicate, a'significant portion of the reactivity feedback occurs during the time that the FRED is in motion. Knowledge of the FRED slug worth and the time dependent FRED behavior (as obtained from the sub-prompt critical familiarization tests and the early portion of all the transients) will allow the feedback reactivity to be calculated in the manner described in section "6.0:3: 

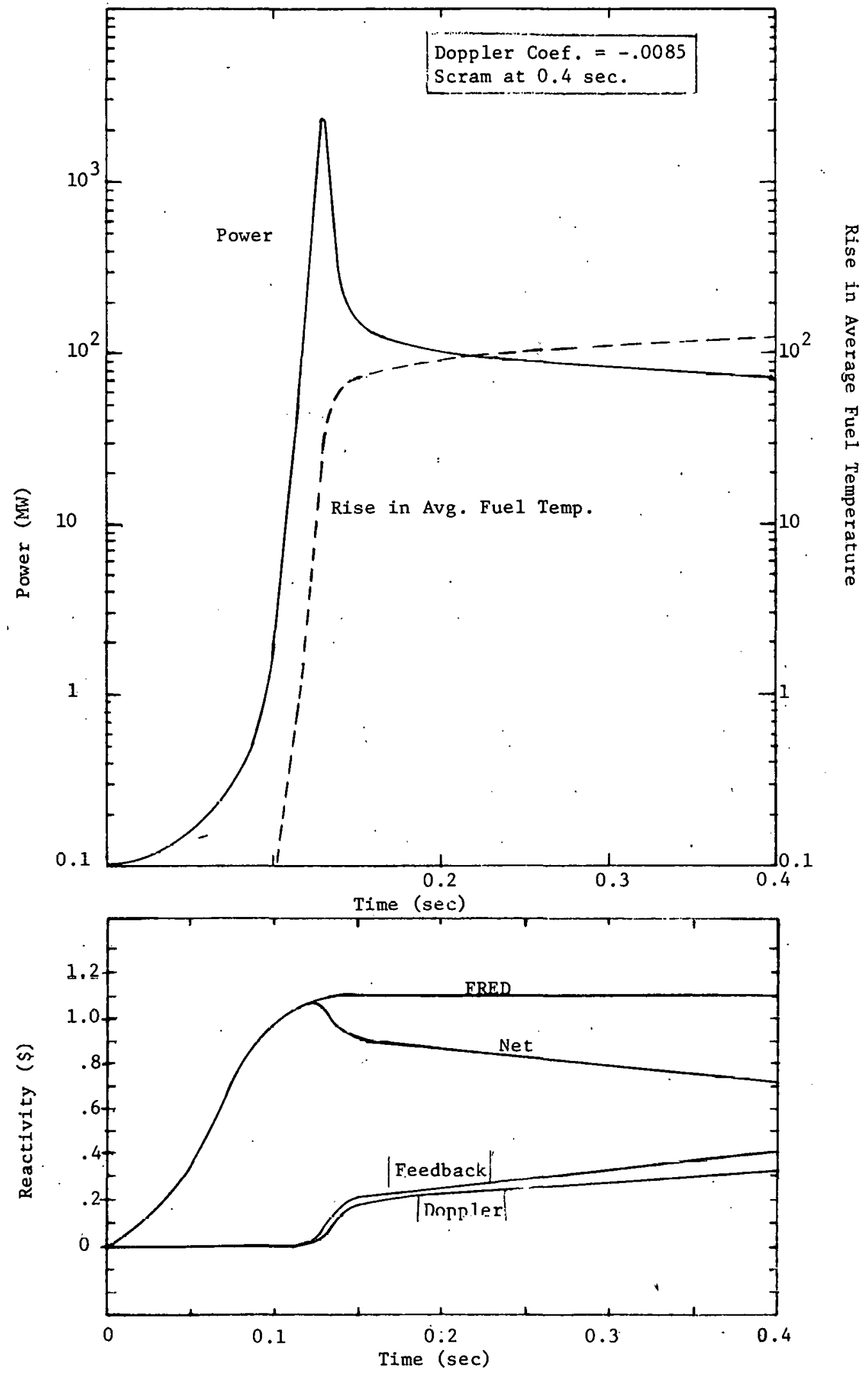

Figure 6.2-1. \$1:10 TRANSIENT AT 0.1 MW FOR CALCULATED COEFFICIENTS 
GEAP -5576
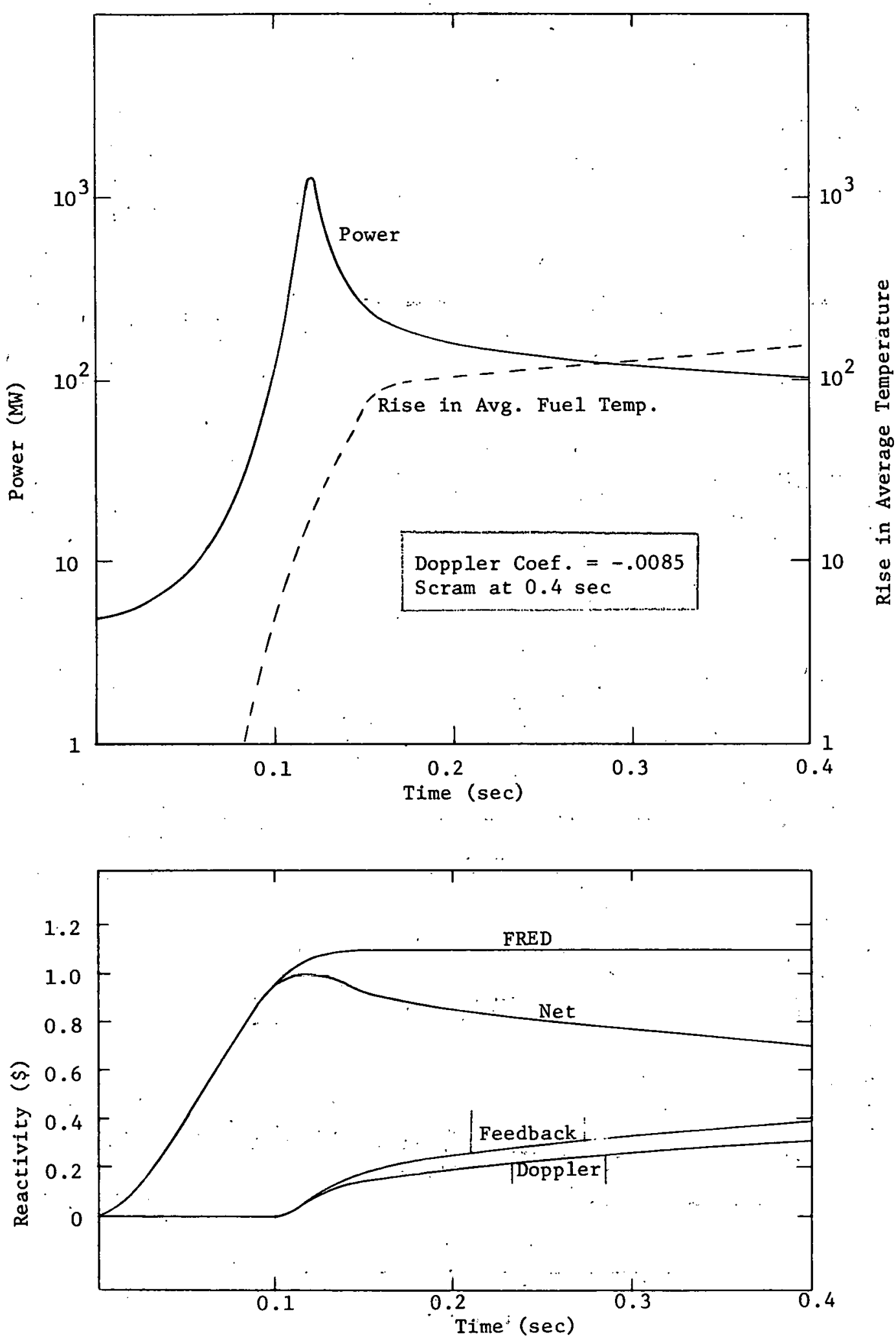

Figure 6.2-2 \$1.10 TRANSIENT AT 5 MW FOR CALCULATED COEFFICIENTS 

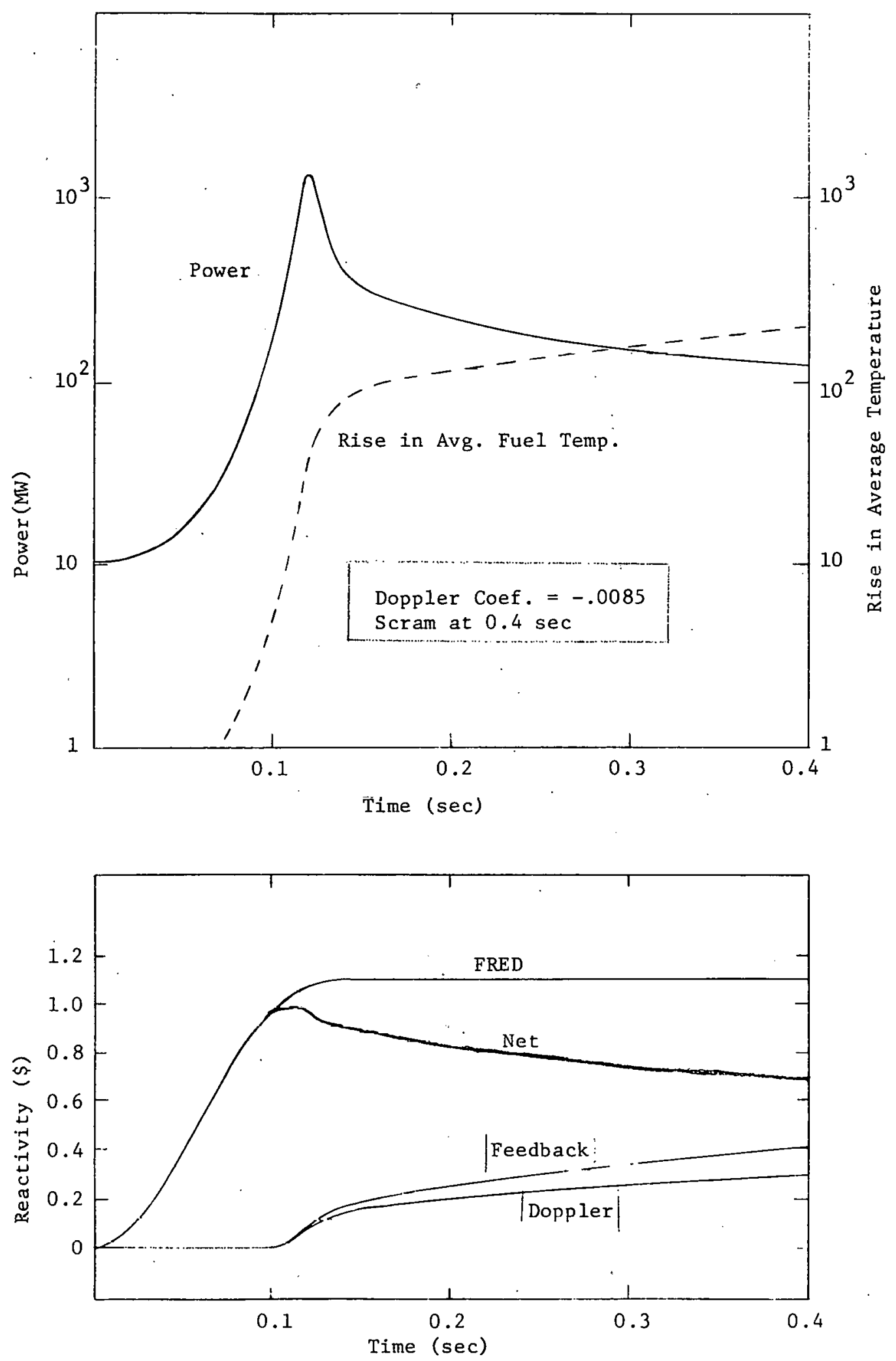

Figure 6.2-3 \$1.10 TRANSIENT AT 10 MW FOR CALCULATED COEFFICIENTS 
GEAP -5576

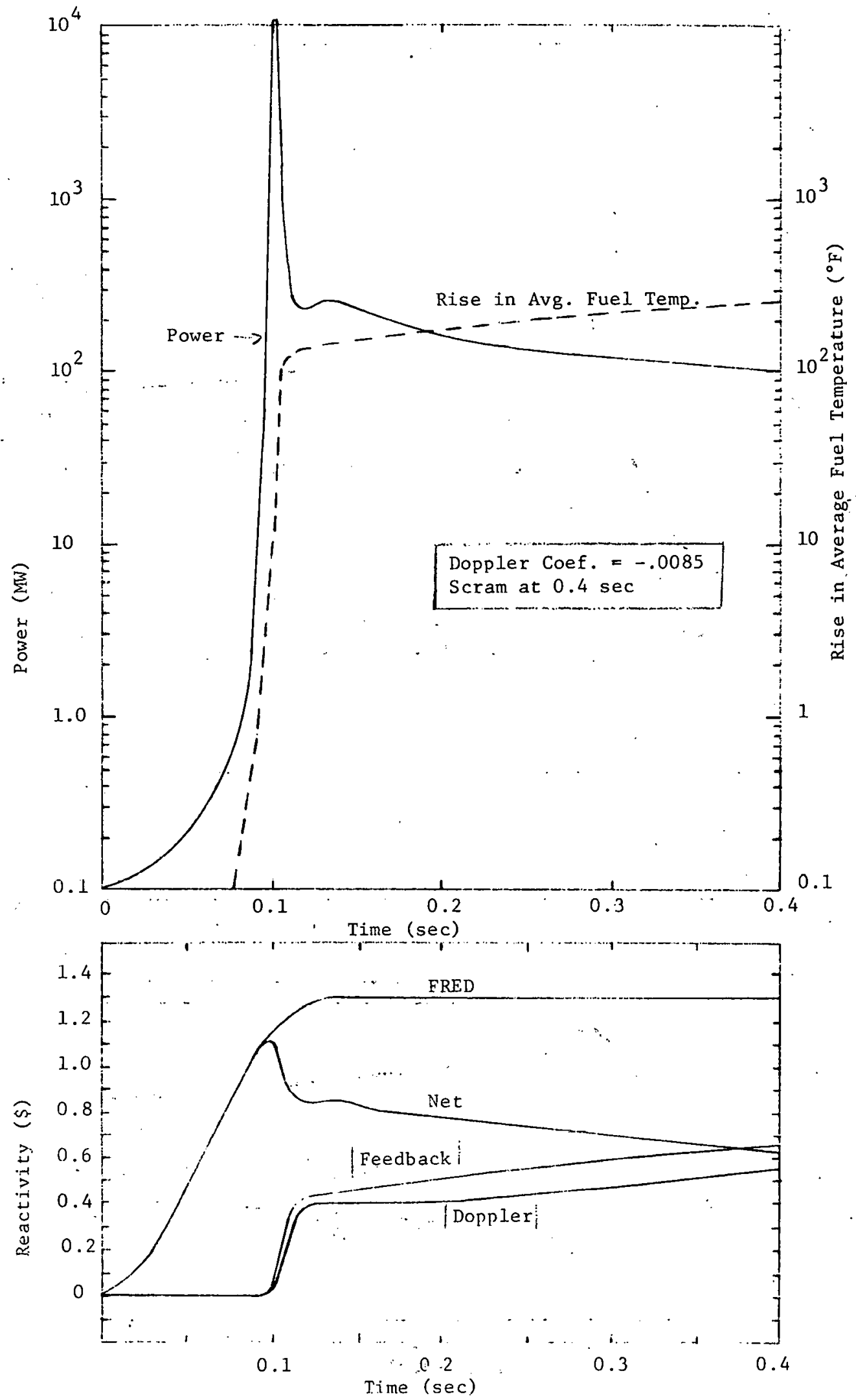

Figure 6.2-4. \$1.30 TRANSIENT AT $0.1 \mathrm{MW}$ FOR CALCULATED COEFFICIENTS 

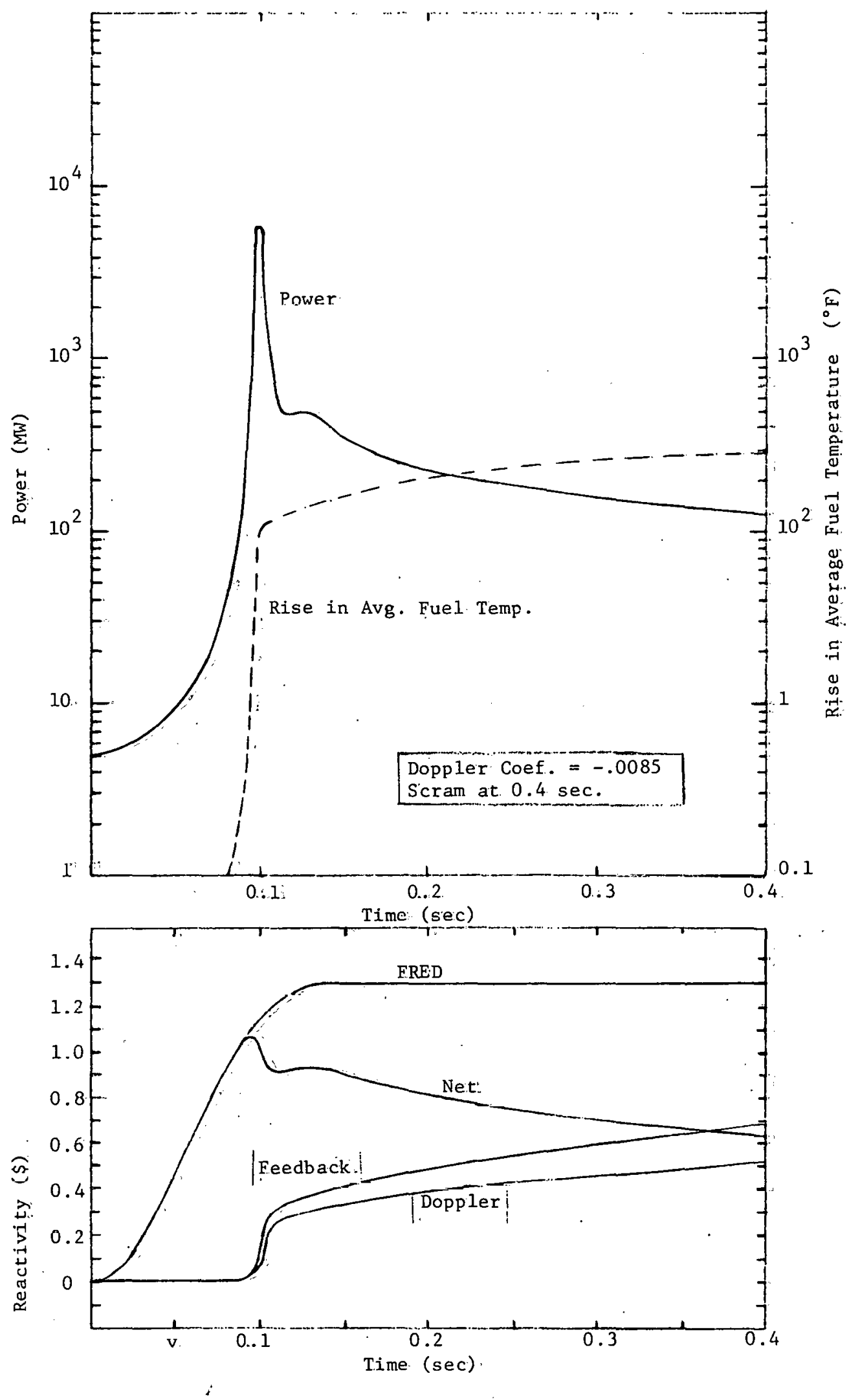

Figure $6.2-5 . \quad \$ 1.30$ TRANSIENT" AT" 5 MW FOR CALCULATED COEFFICIENTS 

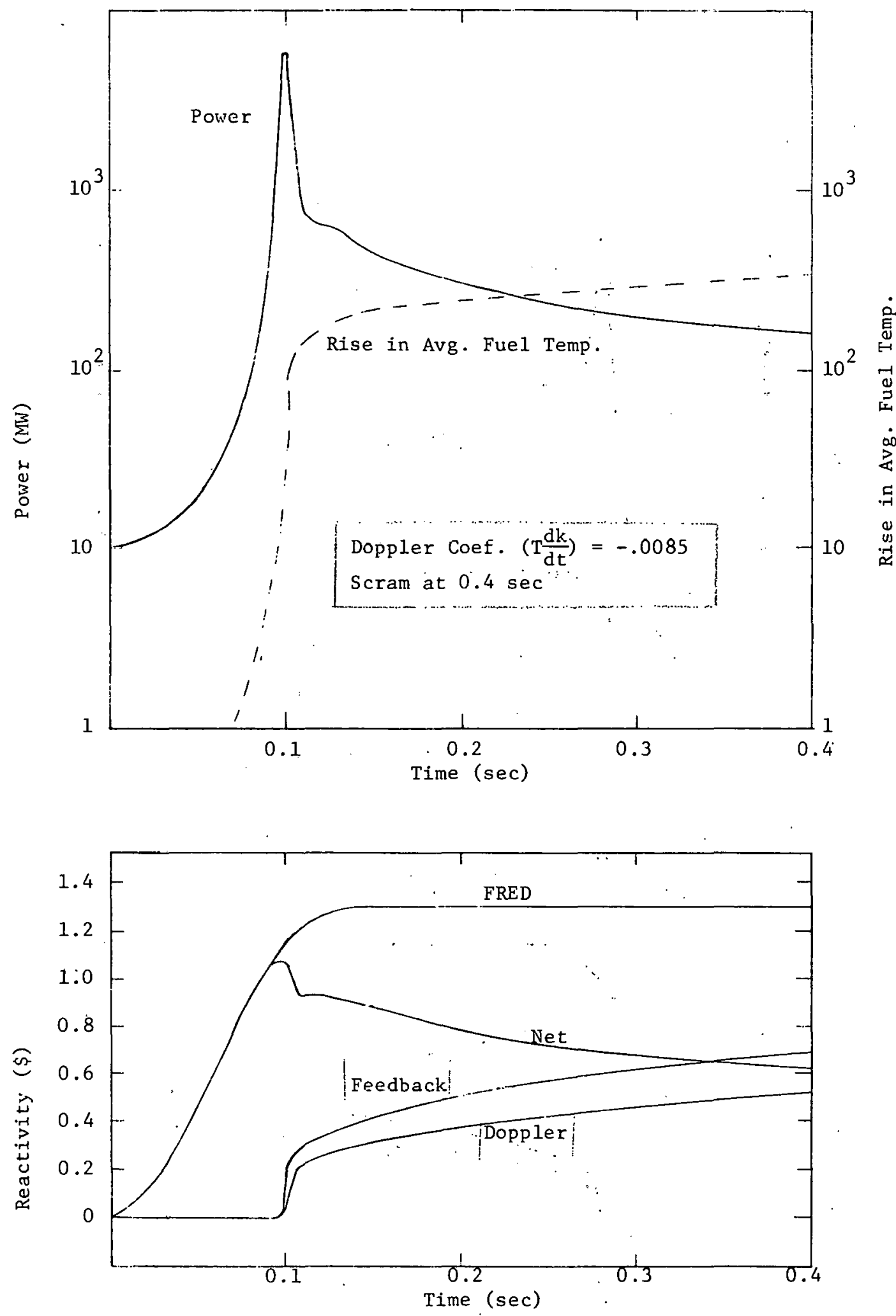

Figure 6.2-6 \$1.30 TRANSIENT AT 10 MW FOR CALCULATED COEFFICIENTS 
GEAP-5576
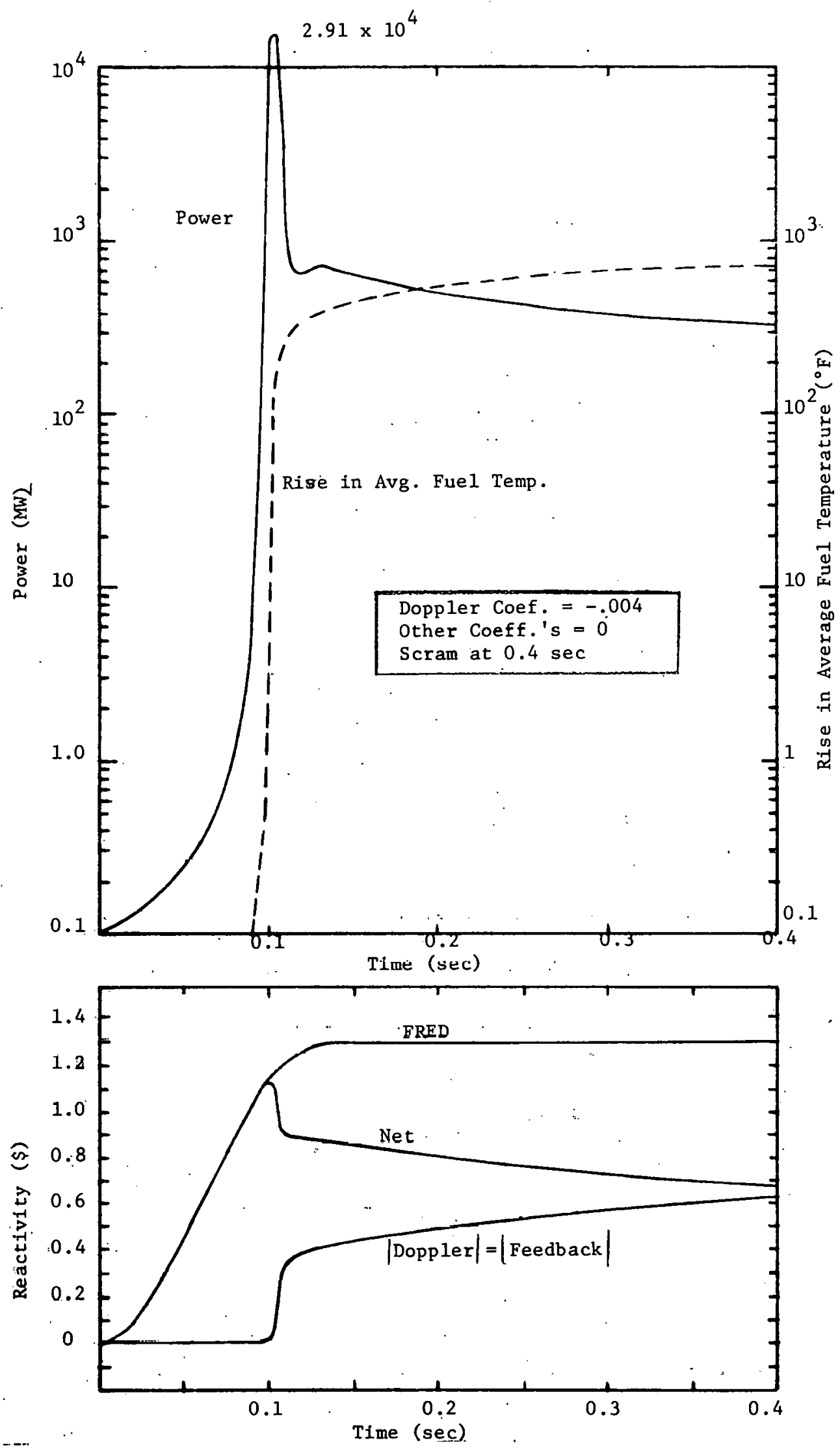

F1gure 6.2-7. \$1.30 TRANSIENT AT 0.1 MW FOR -.004 DOPPLER 
GEAP-5576

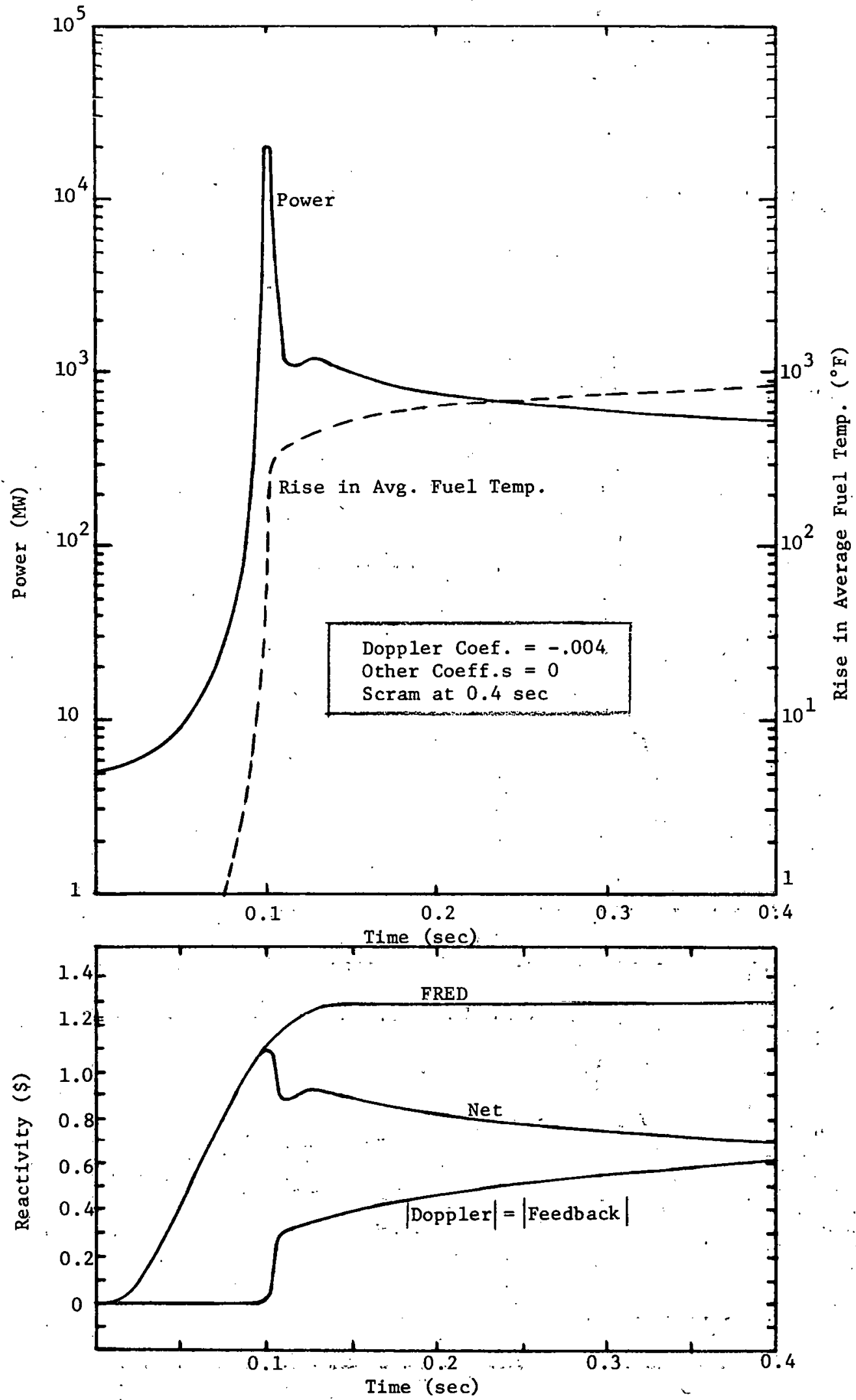

Figure 6.2-8. \$1.30 TRANSIENT AT .5 MW FOR:-.004:DOPPLER 
GEAP-5576
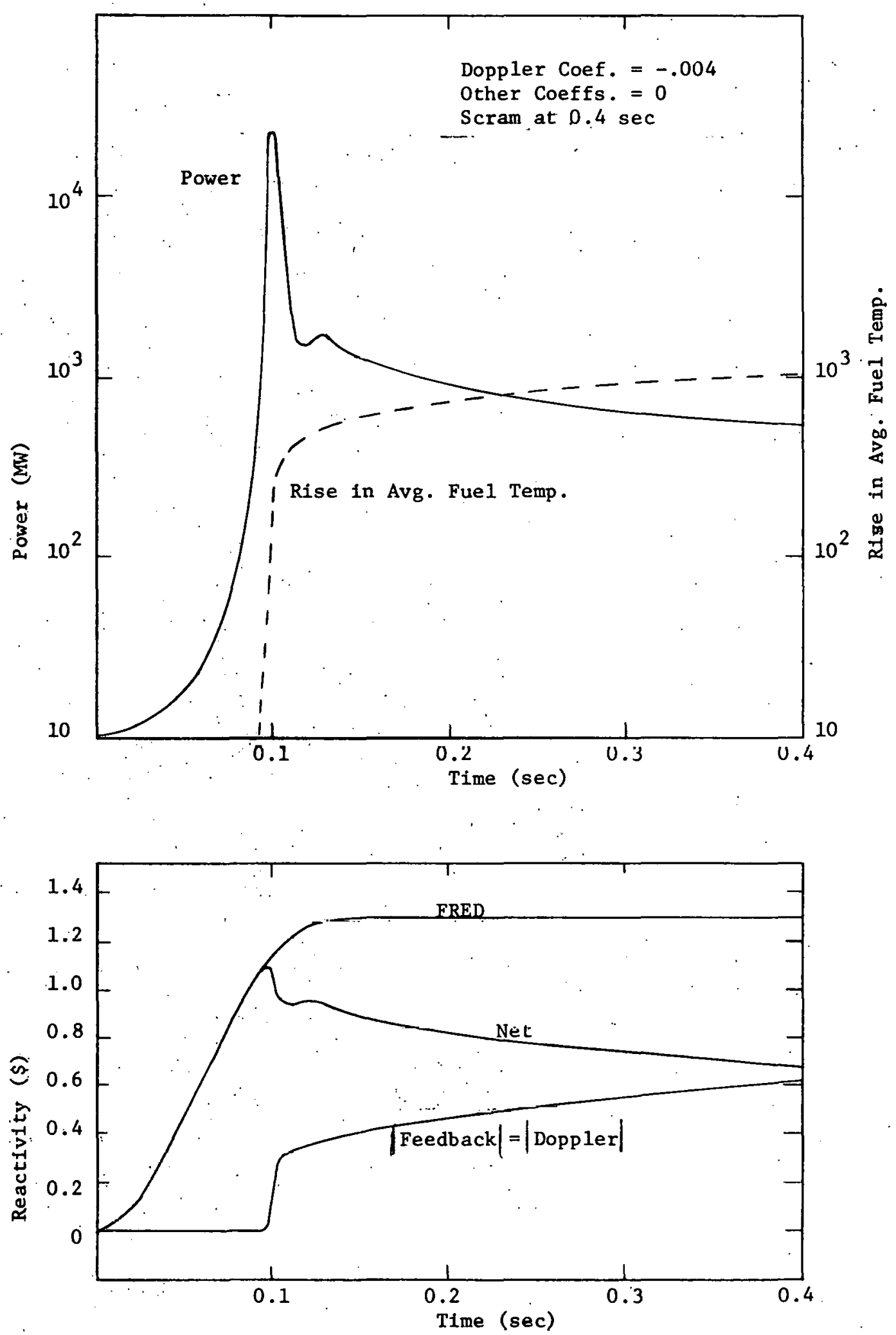

Figure 6.2-9 \$1.30 TRANSIENT AT $10 \mathrm{MW}$ FOR -.004 DOPPLER 
The expanded analytical model shown in Figure 2.5-1, can be simplified by expressing the reactivity feedback effects in terms of power, inlet and outlet temperature oscillations. The simplification can be facilitated by taking the Fourier transform of the composite reactivity model (for small changes in temperature and power) that is described in Section 2.3 .

The transformed equation may be written as:

$$
\Delta K_{f}(j \omega)=C_{f} \Delta T_{f}(j \omega)+C_{f s} \Delta T_{f s}(j \omega)+C_{b} \Delta T_{c}(j \omega)+C_{i} \Delta T_{i}(j \omega)
$$

where it has been assumed that $F_{m}$ (see Figure 2.5-1) $=1.0$ and where

$$
\begin{aligned}
\Delta \mathrm{K}_{\mathrm{f}}(\mathrm{j \omega})= & \begin{array}{l}
\text { transform of total feedback oscillation (corrected } \\
\text { for any effects due to reflector temperature oscil- } \\
\text { lations). }
\end{array} \\
\Delta \mathrm{T}_{\mathrm{f}}(\mathrm{j \omega})= & \begin{array}{l}
\text { transform of "effective" fuel temperature oscillation } \\
\text { (assumed to be the same as the. "average" fuel temper- }
\end{array} \\
& \text { ature): } \\
\Delta \mathrm{T}_{\mathrm{fs}}(j \omega)= & \text { transform of fuel surface temperature oscillation. } \\
\Delta \mathrm{T}_{\mathrm{c}}(j \omega)= & \text { transform of average coolant temperature oscillation. } \\
\Delta \mathrm{T}_{i}(j \omega)= & \text { transform of inlet temperature oscillation. }
\end{aligned}
$$


and where (in terms of the nomenclature of Section 2.3):

$$
\begin{aligned}
& c_{f} \equiv c_{D}+c_{e}(1-a) \\
& c_{f s} \equiv a c_{e}+b c_{t} \\
& c_{b} \equiv c_{c}+c_{t}(1-b)
\end{aligned}
$$

The frequency response of the fuel surface temperature and of the average fuel temperature to power and to coolant temperature oscillations can be written as:

$$
\begin{aligned}
& \Delta T_{f}(j \omega)=K_{a v} F_{a v}(j \omega) X_{f}(j \omega) \Delta P(j \omega)+F_{s}(j \omega) \Delta T_{c}(j \omega) \\
& \Delta T_{f s}(j \omega)=K_{s} F_{s} X_{f}(j \omega) \Delta P(j \omega)+G_{s}(j \omega) \Delta T_{c}(j \omega)
\end{aligned}
$$

where $\mathrm{K}_{\text {av }}$ and $\mathrm{K}_{\mathrm{s}}$ are defined in Section $2,3, \mathrm{~F}_{\mathrm{av}}$ and $\mathrm{F}_{\mathrm{s}}$ are defined in Section 2.5 and where:

$$
\begin{aligned}
G_{s}(j \omega)= & \text { fuel surface temperature response to coolant } \\
& \text { temperature oscillation : } \\
\mathrm{X}_{\mathrm{f}}(j \omega)= & \text { frequency response of the ratio of the total } \\
& \text { power absorbed in the fuel to the "fission" } \\
& \text { power (normalized to unity at zero frequency) } \\
\Delta P(j \omega)= & \text { "fission" power oscillation (proportional to } \\
& \text { neitron flux) }
\end{aligned}
$$


The function $\mathrm{x}_{\mathrm{f}}(\mathrm{j} \omega)$ has been calculated by storrer, $(1)$ and is described in more detail in Appendix II.

In terms of power and coolant temperature oscillations, the reactivity feedback becomes:

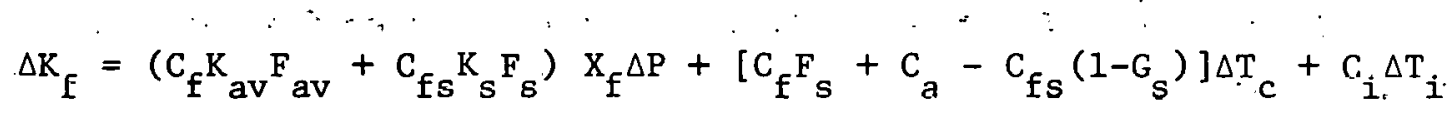

where the average coolant temperature coefficient $\mathrm{C}_{\mathrm{a}}$ is defined by:

$$
c_{a} \equiv c_{b}+c_{f s}=c_{c}+C_{t}+a C_{e}=c_{q}-(1-a) c_{e} \approx c_{q}
$$

Calculations for SEFOR indicate that at a frequency of 0.1 cps an error of $21.5 \%$ amplitude and $20.7 \%$ in phase angle is: introduced into the quantity $C_{f} F_{s}+C_{a}-C_{\tilde{f s}_{s}}\left(1-G_{s}\right)$. if it is assumed that $G_{s}=1.0$. The error at lower frequencies is even smaller. 'Since the uncertainty in $\Delta \mathrm{K}_{f}(j \omega)$ is already large at a frequency of $0.1 \mathrm{cps}$ (see Appendix VI for example), the assumption that $G_{S}(j \omega)=1.0$ intraduces little (if any) loss in the accuracy of the mathematical description of the feedback.

At a frequency of 0.1 cps, the calculated difference between $\mathrm{C}_{\mathrm{f}} \mathrm{K}_{\mathrm{av}} \mathrm{F}_{\mathrm{av}}+\mathrm{C}_{\mathrm{fs}} \mathrm{K}_{\mathrm{s}} \mathrm{F}_{\mathrm{s}}$ and $\left(\mathrm{C}_{\mathrm{f}} \mathrm{K}_{\mathrm{av}}+\mathrm{C}_{\mathrm{fs}} \mathrm{K}_{\mathrm{s}}\right)^{\prime} \mathrm{F}_{\mathrm{av}}$ is $2.2 \%$ in amplitude and $\sim 2.0^{\circ}$ in phase angle. "The difference is even smaller at lower frequencies. 
In view of the above observation, the feedback for the oscillator tests should be adequately approximated by:

$$
\Delta \mathrm{K}_{\mathrm{f}}=\alpha_{\mathrm{f}} \mathrm{F}_{\mathrm{av}} \mathrm{X}_{\mathrm{f}} \Delta \mathrm{P}+\left[\mathrm{C}_{\mathrm{f}} \dot{\mathrm{F}_{\mathrm{s}}}+\mathrm{C}_{\mathrm{q}}\right] \Delta \mathrm{T}_{\mathrm{c}}+\mathrm{C}_{i} \Delta \mathrm{T}_{i}
$$

where, as in the model for the static tests, the power coefficient, $\alpha_{f}$, is defined by:

$$
\alpha_{f} \equiv C_{f} K_{a v}+C_{f s} K_{s}=\alpha_{D}+C_{p}
$$

(note that this implies $\mathrm{K}_{\mathrm{av}}=\mathrm{R}_{\mathrm{f}}$ of Section 2.3.1), and where it has been assumed that $C_{a}=C_{q}$; i.e. that the average coolant coefficient for the static tests is the same as the average coolant coefficient for the oscillator tests.

Although the average coolant temperature oscillation cannot be measured, it is shown in Appendix'III, that it can be expressed in terms of the measurable inlet, $\Delta \mathrm{T}_{i}$, and outlet, $\Delta \mathrm{T}_{0}$, oscillations as:

$$
\Delta T_{c}(i \omega)=F_{i}(j \omega) \Delta T_{i}(j \omega)+F_{0}(j \omega) \Delta T_{0}(j \omega)
$$

For low frequencies $F_{i} \approx F_{0} \simeq 1 / 2$. At higher frequencies, particularly at low. flow rates in the primary coolant system, $F_{0}$ and $F_{i}$ deviate from $1 / 2$. It is expected, however, that $F_{i}$ and $F_{0}$ can be adequately represented by the approximations described in Appendix III. 


\section{ENERGY ABSORPTION IN REACTOR}

\section{1}

\section{REACTOR ENERGY BALANCE}

An energy balance for SEFOR has been calculated by Reynolds. (2): The energy balance is reproduced, in modified form, in Table II- 1 . The "fission" energy absorption includes all energy absorption which is directly proportional to the neutron flux, while, the "decay" energy absorption includes all heating effects caused by the radio-. active decay of fission products. The decay energy shown in the table was calculated for an equilibrium fission product concentration.

If the neutron flux monitors are normalized to $20.0 \mathrm{MW}$ when $20.0 \mathrm{MW}$ is produced in the core (see Table II-1), the absorption rates can be expressed in terms of fractions of the measured power. These fractions are listed in Table II-2 for the materials within the core.

\section{II.2 RESPONSE OF ENERGY ABSORPTION TO A POWER CHANGE}

The total power which is absorbed in the fuel depends on the fission power and upon the decay power. If it is assumed that the flux monitors (which are proportional to the fission power) are normalized to the total steady-state power produced, the power generated in the fuel, $P_{f}$, may be written, using the values, in Table II-2, as follows.

$$
P_{f}(t)=A_{f}\left[0.932 P(t)+0.068 \int_{0}^{\infty} k(u) P(t-u) d u\right]
$$


TABLE II-I:.

\section{REACTOR ENERGY BALANCE}

Basis: 20 MW Produced in Core

Region

Energy Absorption Rate (MW).

"Fission" : Decay : : Tota1...

Fue1

17.58

1.28

18.86

$\mathrm{Na}$, fuel-clad \& structure

0.51

0.19

0.70

$\mathrm{BeO} \& \mathrm{~B}_{4} \mathrm{C}$

TOTAL IN CORE

$\frac{0.11}{18.20}$

$\underline{0.02}$

0.13

18.20

1.49

19.69

Inside Vessel (Non-core)

0.42

TOTAL IN VESSEL

20.11

Outside Vésse1

TOTAL ABSORPTION

0.43

20.54

TABLE $\cdot I I-2$

ENERGY ABSORPTION FRACTIONS

Basis: 20 MW Produced in Core

Fraction of Absorbed

Energy that is

Fraction of

proportional to

Region Energy Absorbed

fission energy

Fraction of Absorbed Energy that is proportional to fission product decay.
Fue1
0.943
0.932
0.068
$\mathrm{Na}, \mathrm{Clad}$
\& Structure
0.035
0.73
0.27
$\mathrm{BeO} \& \mathrm{~B}_{4} \mathrm{C}$
0.0065
0.85
0.15

:. 
where $A_{f}=0.943$ is the fraction of the energy absorbed in the fuel, $P(t)$ is the power indication from the neutron flux monitors, and $k(t-u)$ is the decay kernel.

The kernel $k(u)$ is normalized so that:

$$
\int_{0}^{\infty} k(u) d u=\int_{-\infty}^{\infty} k(u) d u=1.0
$$

where the fact that $k(u)$ is a casual function, i.e. $k(u) \equiv 0$ for $\mathrm{u}<0$, has been used.

For steady-state operation at a constant power, P, equation (1) reduces to:

$$
P_{f}=A_{f} P=0.943 P
$$

in agreement with the values. in Table II-2.

In a similar manner, the total power, $P_{c}$, generated in the coolant, clad, and structure can be written as:

$$
P_{d}(t)=A_{c}\left[0.73 P(t)+0.27 \int_{0}^{\infty} k(u) P(t-u) d u\right]
$$

where $A_{c}=0.035$ is the fraction of energy absorbed in coolant, clad, and structure, and where it has been assumed that the decay kernel for energy absorption in the coolant, clad, and structure is the same as for the energy absorption in the fuel. 
For an oscillator test analysis, the transforms of the power, in the fuel and in the coolant are needed. The transforms can be written : as :

$$
\Delta P_{f}(i \omega)=A_{f} X_{f}(i \omega) \Delta P(i \omega)
$$

and :

$$
\Delta \mathrm{P}_{c}(i \omega)=A_{c} X_{c}(i \omega) \quad \Delta \vec{P}(i \omega)
$$

where $\Delta P_{f}(i \omega), \Delta P_{c}(i \omega)$, and $\Delta P(i \omega)$ are the transforms of the oscillations of the power absorbed in the fuel, the power absorbed in the clad, coolant, and structure, and in the recorded or produced power; respectively.

The quantities $x_{f}(i \omega)$ and $x_{c}(i \omega)$ are defined'by:

$$
\begin{aligned}
& x_{f}(i \omega) \equiv 0.932+0.068 f(i \omega) \\
& x_{c}(i \omega) \equiv 0.73+0.27 f(i \omega)
\end{aligned}
$$

where $f(i \omega)$ is the transform of the kernel $k(t)$.

The function $f(i \omega)$ can be expressed in terms of a function $T(i \omega)$ which has been calculated by storrer ( 1 ) In terms of Storrer's $T(i \omega)$, the function $f(i w)$ is defined as follows:

$$
f(i \omega)=\frac{T(i \omega)-1.0}{T(0)-1.0}
$$


From this definition, $f(0)=1.0$, in agreement with equation (2), and $f(\infty)=0$. Thus,

$$
\begin{aligned}
& x_{f}(0)=x_{c}(0)=1.0 \\
& x_{f}(\infty)=0.932
\end{aligned}
$$

and:

$$
X_{c}(\infty)=0.73
$$

During a rapid transient, the decay power does not change appreclably during the first few seconds and the change in the fuel power can be approximated by:

$$
\Delta P_{f}(t) \simeq A_{f} \cdot 932 \Delta P(t)=A_{f} X_{f}(\infty) \Delta P(t)
$$

The excess energy absorbed in the fuel $\Delta E_{f}(t)$ during the first few seconds after the transient has been initiated can thus be approximated by:

$$
\Delta E_{f} \simeq A_{f} X_{f}(\infty) \int_{0}^{t} \Delta P(u) d u-\dot{A}_{f} X_{f}(\infty) \Delta E(\tau)
$$

where $\wedge F(t)$ is the total excess energy produced.

In order to obtain a more accurate calculation of the energy released into the fuel during a transient an explicit time dependent form of the decay kernel $k(t)$ can be used. One such representation is given by storrer ${ }^{(1)}$ and another is described in reference (3). and is incorporated into the computer code FORE-II. (4)

For times, $t$, up to 10 seconds, the approximation given by (13) agrees quite well (within $0.5 \%$ ) with the results obtained using the kernel described in reference (3). 
GEAP-5576

APPENDIX III

OSCILLATING COOLANT TEMPERATURE KESPONSE

III.1 COOLANT TEMPERATURE IN THE CORE

For small sinusoidal oscillations in power, flow and coolant temperature, the Fourier transform of the heat balance within a differential length of coolant channel can be approximated by:

$$
\begin{aligned}
& \frac{i \omega \tau}{V_{r}} T(x, i \omega)=\frac{a_{c} k_{n} x_{c}(i \omega)}{V_{r}} P(i \omega) q(x) \\
& +\frac{K_{n}\left(1-a_{c}\right)}{K_{s} V_{r}}\left[T_{s}(x, i \omega)-T(x, i \omega)\right] \\
& -\frac{d}{d x} T(x, i \omega)-\frac{v(i \omega)}{V_{0}} \frac{d \theta(x)}{d x}
\end{aligned}
$$

where $1=\sqrt{-1}, \omega$ is the frequency of oscillation in radians per second, and where:

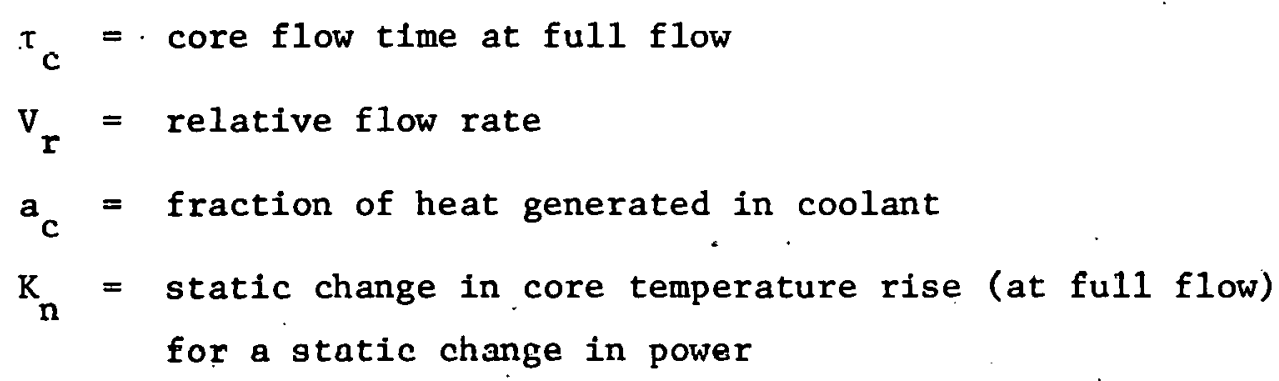




$$
\begin{aligned}
& \mathrm{K}_{\mathbf{S}}=\text { static change in fuel surface for a power change } \\
& \text { at constant coolant temperature } \\
& v_{0}=\text { mean flow rate }=\text { Product of } v_{r} \text { and full flow rate } \\
& T(x, f \omega)=\text { transform of the coolant temperature oscillation } \\
& P(i \omega)=\text { transform of the prompt power oscillation (proportional } \\
& \text { to flux) } \\
& x_{c}(i \omega)=\text { transform of ratio of total power generated in coolant. } \\
& \text { (p̈roportional to prompt power pius delayed gammas due } \\
& \text { to fission product decay) to the prompt power. } \\
& T_{s}(x, i \omega)=\text { transform of the fuel surface temperature oscillation } \\
& V(i \omega)=\text { transform of the flow oscillation } \\
& \theta(x)=\text { static coolant temperature at axial position } x \text { and } \\
& \partial \theta / \partial x=K_{n} P_{0} q / V_{r} \text { where } P_{0} \text { is the mean power level } \\
& x \text { - normalized axial position } \\
& q(x)=\text { axial power distribution (assumed to be the same in } \\
& \text { coolant and fuel) normalized so that: } \\
& \int_{0}^{1} q(x) d x=1.0
\end{aligned}
$$

According to reference $(5), \mathrm{T}_{\mathrm{S}}$ can be written, after slight modification, as :

$$
T_{s}(x, i \omega)=K_{s} F_{s}(i \omega) X_{f}(i \omega) P(i \omega) q(x)+\left[1-i \omega \gamma F_{s}(i \omega)\right] T(x, i \omega)
$$

where $F_{S}(i \omega)$, and $\gamma$ are defined in Section 2.5 , and $x_{f}(i \omega)$ is the transform of the ratio of the total power generated in the fuel to the prompt power generated in the fuel. (See Appendix II) 
Equation (1) can thus be written as:

$$
\frac{\partial T(x, i \omega)}{\partial x}+b(i \omega) T(x, i \omega)=S \cdot(i \omega) q(x)
$$

where:

$$
b(i \omega) \equiv \frac{i \omega}{V_{r}}\left(\tau_{c}+\gamma K_{n}\left(1-a_{c}\right) F_{s} / K_{s}\right)
$$

and:

$$
S(i \omega) \equiv \frac{K_{n} P_{0}}{V_{r}}\left[\left\{a_{c} x_{c}(i \omega)+\left(1-a_{c}\right) F_{s}(i \omega) x_{f}(i \omega)\right\} \frac{P(i \omega)}{P_{0}}-v(i \omega) / V_{0}\right]
$$

The solution of equation (2) is:

$$
\mathrm{T}=\mathrm{T}_{i} \mathrm{e}^{-\mathrm{bx}}+\mathrm{S} \int_{0}^{\mathrm{x}}: \mathrm{q}(\mathrm{z}) \mathrm{dz} \exp [-\mathrm{b}(\mathrm{x}-\mathrm{z})] \because \ldots
$$

where $T_{i} \equiv T(0, i \omega)$.

The average coolant temperature, which is defined by:

$$
\bar{T}(i \omega)=\int_{0}^{1} T(x, i \omega) d x
$$

can be written as:

$$
\overline{\mathrm{T}}=\mathrm{T}_{i}\left(1-\mathrm{e}^{-\mathrm{b}}\right) / \mathrm{b}+\frac{\mathrm{s}}{\mathrm{b}} \int_{0}^{1} \mathrm{q}(\mathrm{z}) \mathrm{d} z[1-\exp (\mathrm{bz-b})]
$$


The average temperature can also be written in terms of the inlet temperature oscillation $T_{i}$ and the outlet temperature oscillation $\mathrm{T}_{0}$ as follows:

$$
\bar{T}=F_{i} T_{i}+F_{0} T_{0}
$$

where $F_{i} \equiv(I-1) / b I$, and $F_{0} \equiv\left(e^{b}-1\right) / b I$, and where

$$
I \equiv \int_{0}^{\hat{1}} q(z) e^{b z} d z
$$

For $|b| \ll 1$, the expression for $F_{i}$ and $F_{0}$ can be approximated by :

$$
\begin{aligned}
& \dot{F}_{0} \simeq 1-F_{i} \\
& F_{i} \simeq \frac{1}{2}(1-b \alpha)
\end{aligned}
$$

where it has been assumed that $q(x)$ is symmetric about $x=\frac{1}{2}$ and where the parameter $\alpha$ is defined:

$$
\alpha \equiv \frac{1}{2}-\int_{0}^{1} \dot{x}^{2} q(x) d x
$$

For a parabolic power distribution $\alpha=(3+2 r) / 30$, where $r$ is the axial peaking factor. 
Values of the functions $F_{i}$ and $F_{0}$ have been calculated for several cases using SEFOR parameters and an assımed parabolic power distribution. These are shown in Figures III-1 and III-2. At low flow rates, the approximate deviation in amplitude from 0.5 and the deviation in phase from zero degrees varies inversely as the relative flow rate. The approximate expressions for $F_{i}$ and $F_{0}$ (equations (6) and (7)) are compared with the more accurate expressions in Figures III-3 and III-4.

It is seen from Figure III-1 and III-2, that the functions $F_{0}$ and $F_{i}$ deviate even at full flow from the zero frequency value of 0.5 for frequencies above $0.01 \mathrm{rad} / \mathrm{sec}(0.0016 \mathrm{cps})$. Even at low flow rates $(30 \%)$, however, the approximate expression agrees we11 with the more accurate expression up to frequencies of $\sim 0.6 \mathrm{rad} / \mathrm{sec}$ ( 0.1 cps $)$. As the expected feedback is nearly zero above $0.1 \mathrm{cps}$, the approximate expressions, (6) and (7), can be used to calculate the functions $F_{i}$ and $F_{o}$. The "measured" average coolant temperature is then calculated from (5) using measured values of inlet and outlet cuolanl cemperature oscillations.

A mathematical expression for the ratio of the coolant flow oscillation to the power oscillation is needed for the analysis of the first balanced oscillator test data. This expression (obtained by integrating (2) over $x$ ) can be written as follows:

$$
\begin{aligned}
\frac{\frac{V(i \omega)}{V_{o}}}{\underbrace{P(i \omega)}_{0}}=a_{c} x_{c}+\left(1-a_{c}\right) F_{s} x_{f}-\frac{P o}{\Delta \theta}\left[\frac{T_{0}-T_{i}+b \bar{T}}{P}\right] \\
=a_{c} x_{c}+\left(1-a_{c}\right) F_{s} X_{f}-\frac{P o}{\Delta \theta}\left[\frac{T_{o}}{P}\left(1+b-b F_{i}\right)-\frac{T_{i}}{P}\left(1-b F_{i}\right)\right]
\end{aligned}
$$

where $\Delta \theta$, the steady-state core temperature rise is defined by $\Delta \theta=K_{n} P_{0} / V_{r}$. 


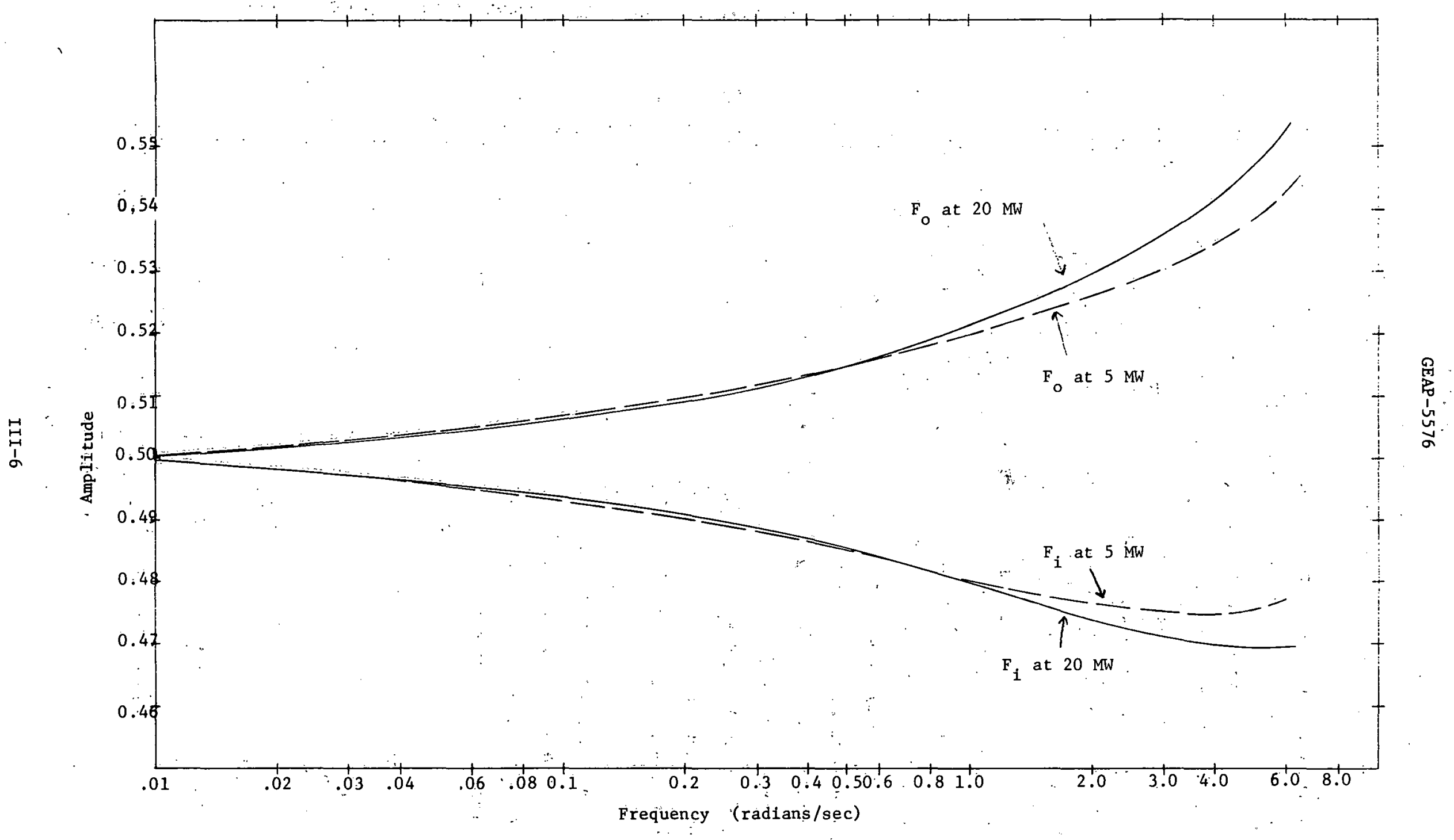

Figure III-1. AMPLITUDE OF THB COOLANT TEMPERATURE FUNCTIONS $\vec{F}_{1}$ AND F FOR SEFOR AT 10O\% FLOW 


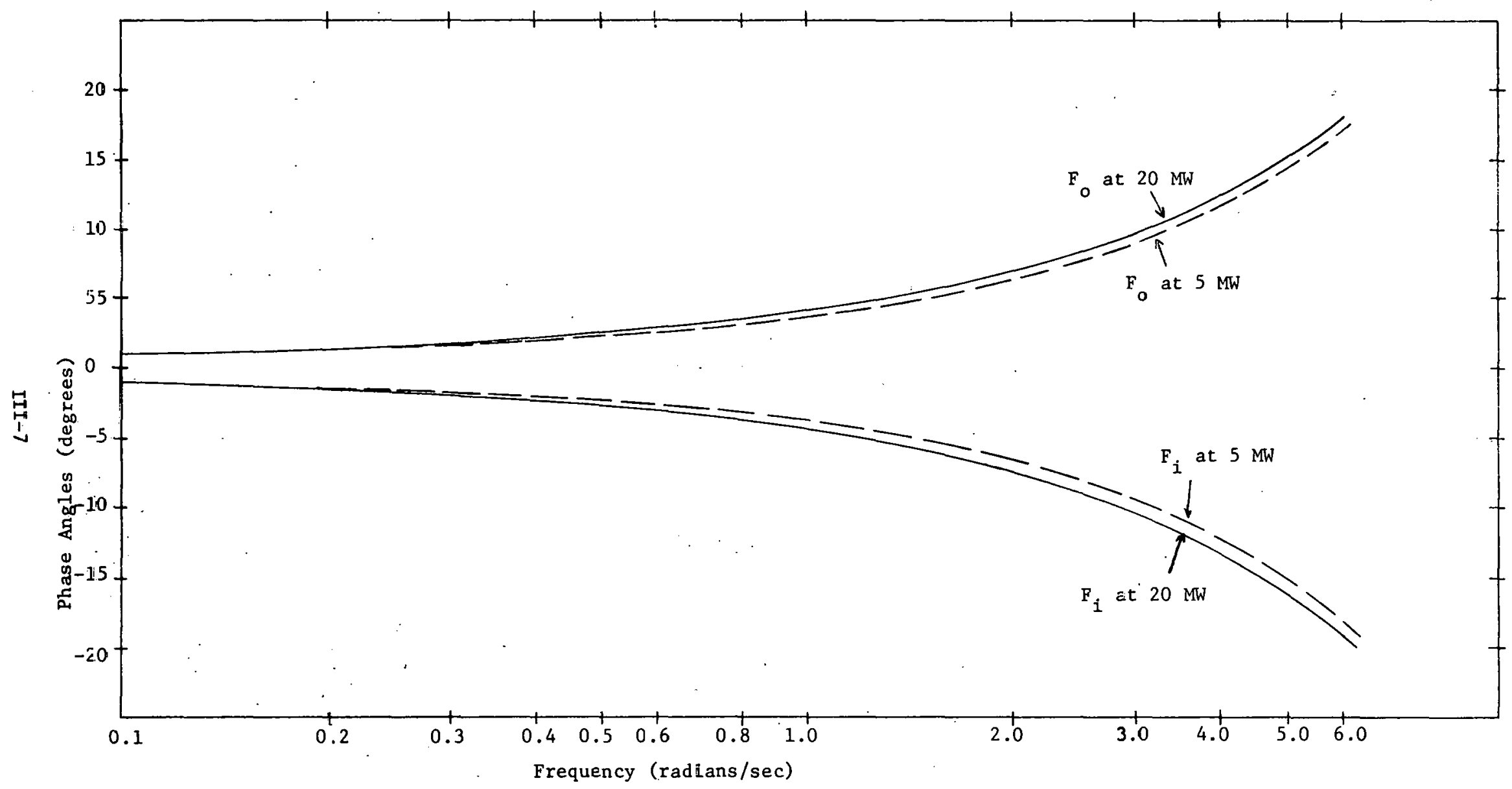

FIgure ILI $\div 2$. PHASE AUGGE OF THE COOLANT TEMPERATURE FUNCTIONS $F_{1}$ AND $F_{0}$ FOR SEFOR AT $100 \%$ FLOW 


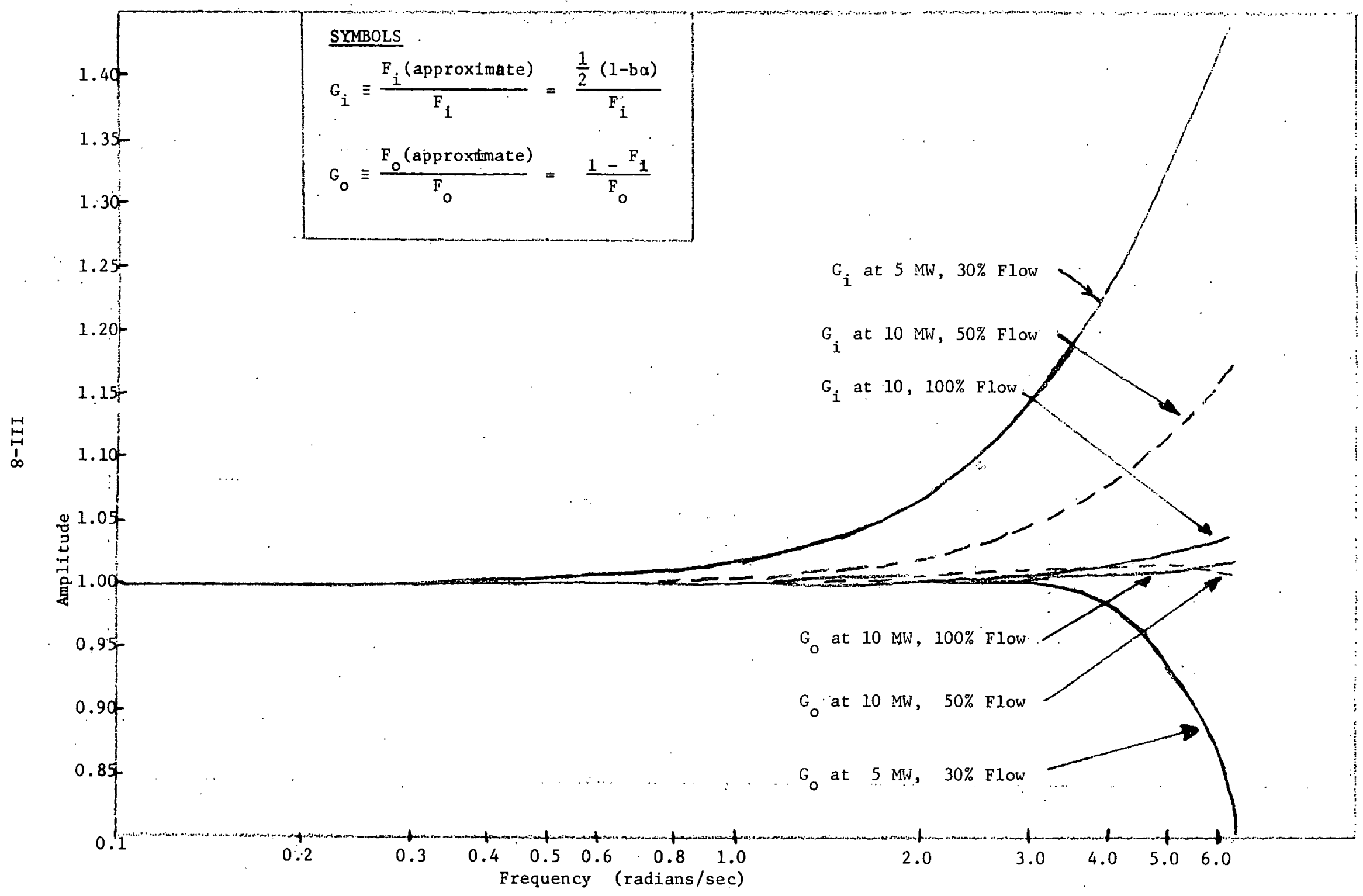

Figure III-3. ERROR IN AMPLITUDE OF APPROXIMATE EXPRESSIONS FOR $F_{1}$ AND $F_{0}$ IN SEFOR 


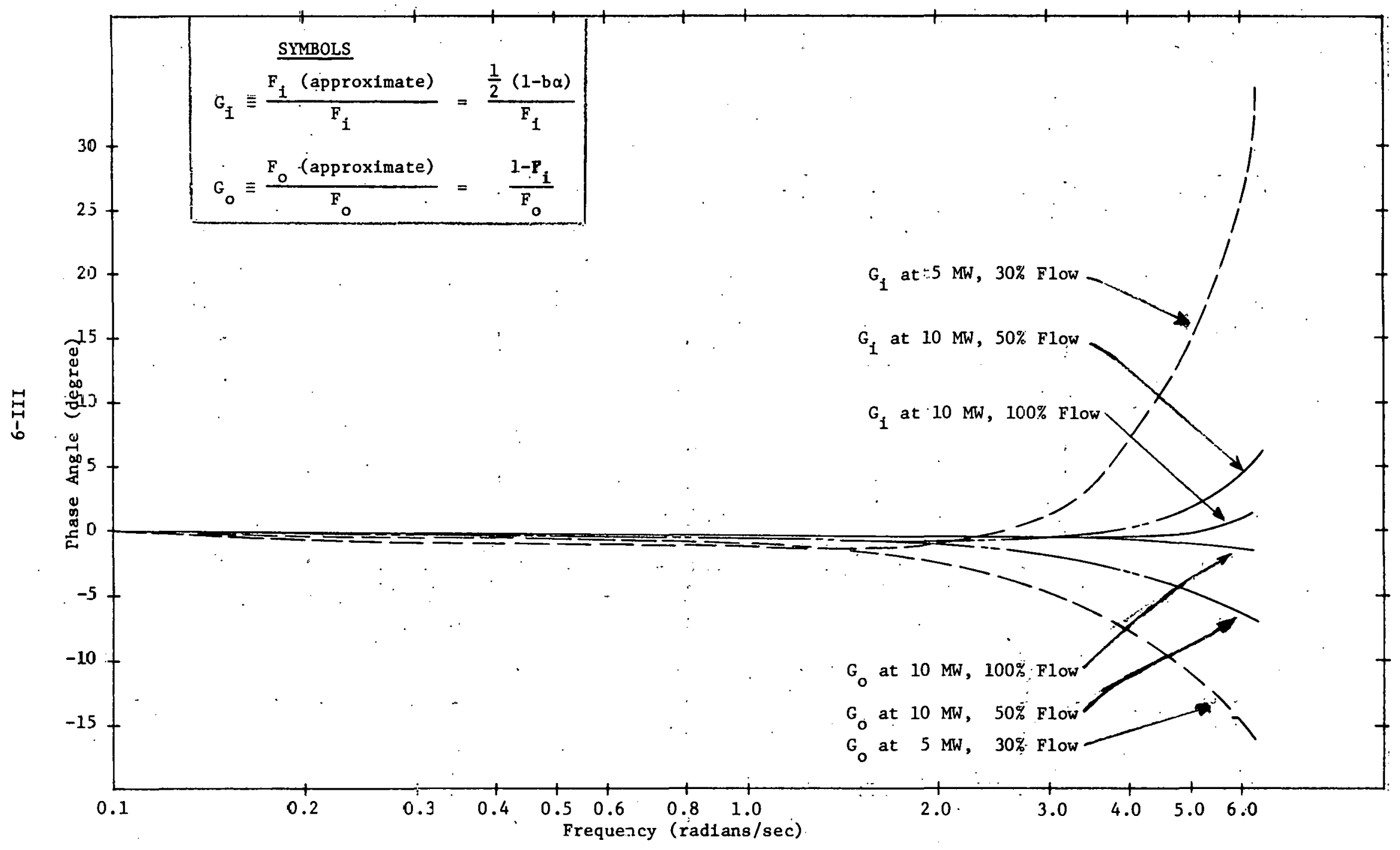

Figure III-4. ERROR IN PHASE ANGLE OF APPROXIMATE EXPRESSIONS FOR $F_{1}$ AND $F_{0}$ IN SEFOR 


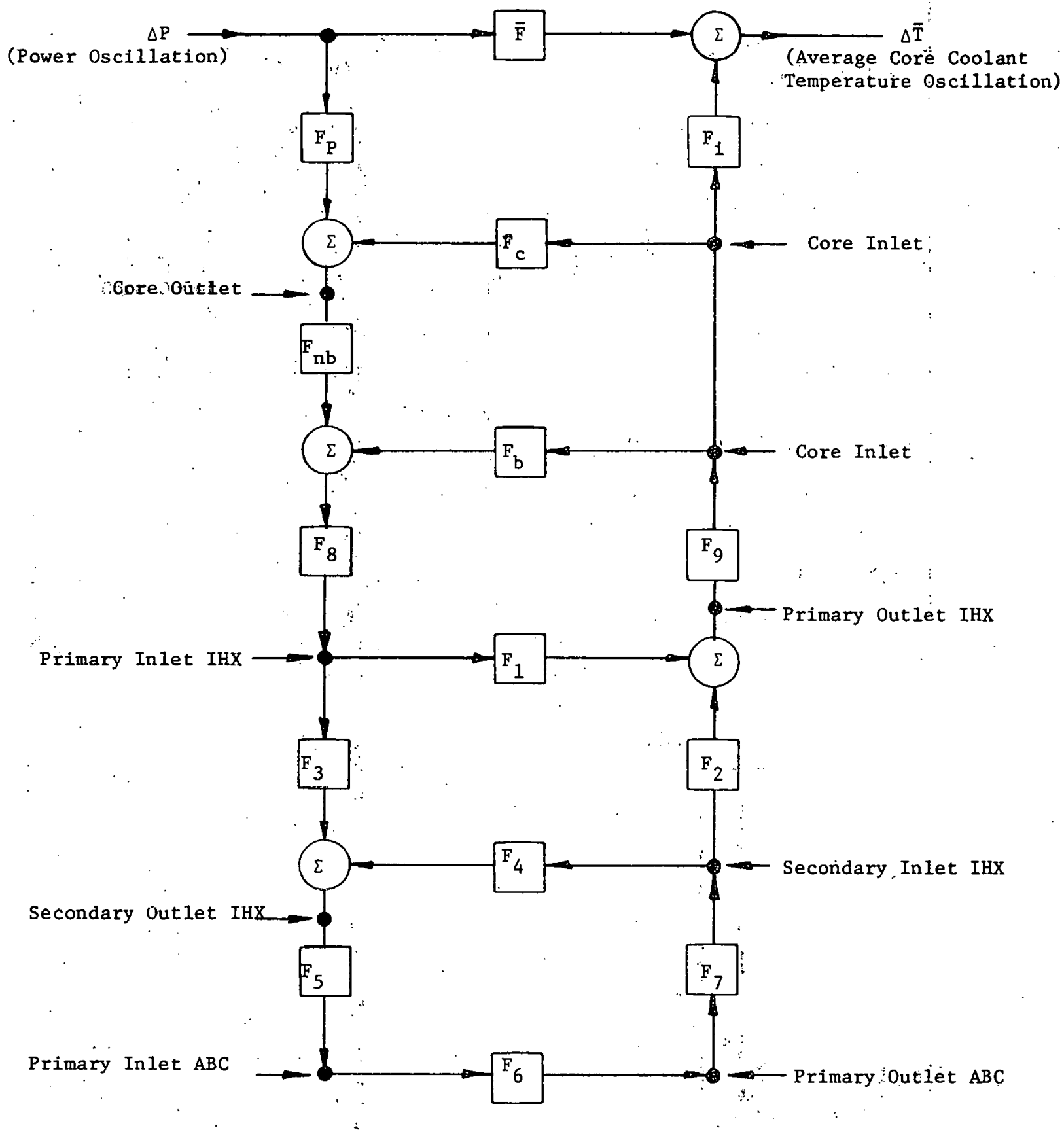

Figure III-5. MODEL FOR FREQUENCY RESPONSE OF AVERAGE COOLANT TEMPERATURE TO A POWER OSCILLATION 


\section{III.2 COOLANT TEMPERATURES IN EXTERNAL LOOP}

\section{Heat Exchangers}

In a conventional oscillator test no attempt is made to hold the coolant temperatures constant. The response of the power to a reactivity oscillation will thus be influenced by the frequency response of the entire coolant system.

In order to obtain an estimate of the frequency response of the power-reactivity transfer function for the conventional oscillator tests, the model for the SEFOR coolant system that is described by Russe11 ${ }^{(6)}$ was used. This model assumes counter-flow heat exchangers rather than the actual tube and shell heat exchangers. The model was modified, as described below, to allow for a continuous axial temperature distribution through the heat exchangers, and to allow the system to be analyzed directly in the frequency rather than the time domain.

For small sinusoidal oscillations in flow and temperature, the Fourier transform of the heat balance within a differentlal length of a counter-flow heat exchanger can be written as:

$$
\begin{aligned}
& \frac{C_{1} \partial T_{1}}{\partial x}(x, i \omega)=\left(1+i \omega \tau_{1}\right) T_{1}(x, i \omega)-\frac{C_{1} \Delta V_{1}(i \omega)}{V_{10}} \frac{\partial \theta_{1}(x)}{\partial x}-T_{3}(x, i \omega) \\
& C_{2} \frac{\partial T_{2}}{\partial x}(x, i \omega)=-\left(1+i \omega \tau_{2}\right) T_{2}(x, i \omega)+T_{3}(x, i \omega)-\frac{C_{2} \Delta V_{2}(i \omega)}{V_{20}} \frac{\partial \theta_{2}(x)}{\partial, x} \\
& T_{3}(x, i \omega)=\frac{a T_{1}(x, i \omega)+(1-a) T_{2}(x, i \omega)}{1+i \omega \tau} 3
\end{aligned}
$$


where $i=\sqrt{-1}, \omega$ is the frequency of oscillation in cycles per second, $x$ is the dimensionless length and where:

$$
\begin{aligned}
& T_{1}(x, i \omega)=\text { transform of coolant temperature oscillation } \\
& \text { on the primary side } \\
& \mathrm{T}_{2}(\mathrm{x}, \mathrm{i} \omega)=\text { transform of coolant temperature oscillation } \\
& \text { on the secondary side } \\
& \mathrm{T}_{3}(\mathrm{x}, \mathrm{i} \omega)=\text { transform of the temperature oscillation in } \\
& \text { the heat exchanger wall between the primary } \\
& \text { and secondary coolant } \\
& \Delta V_{j}(i w)=\text { transform of the fractional coolant temperature } \\
& \text { oscillation on the primary: }(j=1) \text { and secondary } \\
& (j=2) \text { side } \\
& \dot{\theta}_{j}(x)=\text { steady-state coolant temperature distribution } \\
& \text { on the primary }(j=1) \text { and secondary }(j=2) \text { side } \\
& \tau_{\mathrm{j}} / \mathrm{C}_{\mathrm{j}, \cdots}=\text { coolant flow time through the heat exchanger } \\
& \text { in the primary }(j=1) \text { and secondary }(j=2) \text { side } \\
& \text { a = fraction of steady-state wall temperature that } \\
& \text { depends on the steady-state coolant temperature } \\
& \text { on the primary side } \\
& \mathrm{r}_{j}=\text { time constant for the decay of a coolant temper- } \\
& \text { ature perturbation at "zero" flow on the primary } \\
& (j=1) \text { and secondary }(j=2) \text { side }
\end{aligned}
$$


The solutions for $\frac{\partial \theta_{1}}{\partial x}$ and $\frac{\partial \theta_{2}}{\partial x}$ can be written as:

$$
\frac{\partial \theta_{1}}{\partial x}=g_{1} e^{p x}
$$

and

$$
\frac{\partial \theta_{2}}{\partial x}=g_{2} e^{p x}
$$

where $p=(1-a) / c_{1}-a / c_{2} ;$ where

$$
g_{1}=\frac{1-a}{C_{1}}\left(\theta_{10}-\theta_{21}\right)
$$

where $g_{2}=a c_{1} g_{1} / c_{2}(1-a)$, and where $\theta_{10}$ is the steady-state outlet temperature on the primary side, and $\theta_{2 i}$ is the steady-state inlet temperature on the secondary side.

Defining:

$$
\begin{aligned}
& \mu_{1}=\frac{1}{c_{1}}\left(1+i \omega \tau_{1}\right)-\frac{a}{c_{1}\left(1+i \omega \tau_{3}\right)} \\
& \mu_{2}=-\frac{1}{c_{2}}\left(1+i \omega \tau_{2}\right)+\frac{(1-a)}{c_{2}\left(1+i\left(\omega \tau_{3}\right)\right.} \\
& \alpha_{1}=\frac{(1-a)}{c_{1}\left(1+i \omega \tau_{3}\right)} \\
& \alpha_{2}=\frac{a}{c_{2}\left(1+i \omega \tau_{3}\right)}
\end{aligned}
$$


equations (9) and (10) can be written as:

$$
\begin{aligned}
& \frac{\partial \mathrm{T}_{1}}{\partial \mathrm{x}}=\mu_{1} \mathrm{~T}_{1}-\alpha_{1} \mathrm{~T}_{2}-\frac{\Delta \mathrm{V}_{1}}{\mathrm{~V}_{1 o}} \mathrm{~g}_{1} e^{\mathrm{px}} \\
& \frac{\partial \mathrm{T}_{2}}{\partial \mathrm{x}}=\mu_{2} \mathrm{~T}_{2}+\alpha_{a} \mathrm{~T}_{1}-\frac{\Delta \mathrm{V}_{2}}{\mathrm{~V}_{2 o}} \mathrm{~g}_{2} e^{\mathrm{px}}
\end{aligned}
$$

The roots, $\mathrm{S}_{1}$ and $\mathrm{S}_{2}$, of the characteristic equation are:

$$
\begin{gathered}
S_{1}(i \omega)=\frac{\mu_{1}+\mu_{2}}{2}+\frac{1}{2} \sqrt{\left(\mu_{1}-\mu_{2}\right)^{2}-4 \alpha_{1} \alpha_{2}} \\
\vdots \\
s_{2}(i)=\frac{\mu_{1}+\mu_{2}}{2}-\frac{1}{2} \sqrt{\left(\mu_{1}-\mu_{2}\right)^{2}-4 \alpha_{1} \alpha_{2}}
\end{gathered}
$$

The transform of the outlet coolant temperature oscillation on the primary side, $T_{p o}$, and on the secondary side, $T_{\text {so' }}$ ' can thus be written in terms of the transform of the inlet temperature oscillations on the primary side, $\mathrm{T}_{\mathrm{pi}}$, the secondary side, $\mathrm{T}_{s i}$, and in terms of the coolant flow oscillations as follows:

$$
\begin{gathered}
T_{p o}=F_{1} T_{p i}+F_{2} T_{s i}+G_{1} \frac{\Delta V_{1}}{V_{10}}+G_{2} \frac{\Delta V_{2}}{V_{20}} \\
T_{s o}=F_{3} T_{p i}+F_{4} T_{s i}+G_{3} \frac{\Delta V_{1}}{V_{10}}+G_{4} \frac{\Delta V_{2}}{V_{20}}
\end{gathered}
$$


where:

$$
\begin{aligned}
& F_{1}=\left(S_{1}-S_{2}\right) / D \\
& F_{2}=\alpha_{1}\left(e^{S_{1}}-e^{S_{2}}\right) / D \\
& F_{3}=\alpha_{2}\left(e^{S_{I}}-e^{S_{2}}\right) / D \\
& F_{4}=\left(S_{1}-S_{2}\right) \cdot e^{S_{1}} e^{S_{2} / D} \\
& G_{1}=\frac{g_{1}}{D}\left[\frac{\left(S_{1}-\mu_{2}\right)}{\left(P-S_{1}\right):}\left(e^{P}-e^{S_{1}}\right)-\frac{\left(S_{2}-\mu_{2}\right)}{\left(P-S_{2}\right)} \cdot\left(e^{P}-e^{S_{2}}\right)\right] \\
& G_{2}=\frac{-\alpha_{1} g_{2}}{D}\left[\frac{e^{P}-e^{S_{1}}}{P-S_{1}}-\frac{e^{P}-e^{S_{2}}}{P-S_{2}}\right] \\
& G_{3}=\frac{-\alpha_{2} g_{1}}{D}\left[e^{S_{2}} \frac{\left(e^{P}-e^{S_{1}}\right)}{P-S_{1}}-e^{S_{1}} \frac{\left(e^{P}-e^{S_{2}}\right)}{P-S_{2}}\right] \\
& G_{4}=\frac{g_{2}}{D}\left[\left(S_{2}-\mu_{2}\right) e^{S_{2}} \frac{\left(e^{P}-e^{S_{1}}\right)}{P-S_{1}}-\frac{\left(S_{1}-\mu_{2}\right)}{\cdots} \cdot \frac{e^{S_{1}\left(e^{P}-e^{S_{2}}\right)}}{P-S_{2}}\right]
\end{aligned}
$$

where: $D=e^{S_{1}}\left(S_{1}-\mu_{2}\right)-e^{S_{2}}\left(S_{2}-\mu_{2}\right)$

The block diagram for a portion of the modified form of Russell's (6) model is shown in Figure III-5. In this diagram, it is assumed that the inlet temperature of the air blast coolant and all flow rates are constant. The frequency response $(\Delta \overline{\mathrm{T}} / \Delta \mathrm{P})$ of the average coolant temperature to a power oscillation that is obtained from the model shown in Figure III-5 was used in the feedback equations described in Section 2.5 to calculate the power-reactivity transfer function for the conventional. oscillator test $\left(\Delta \mathrm{V}_{1}=\Delta \mathrm{V}_{2}=0\right)$ shown in Section 5.2.4. The various response functions shown in the diagram are defined below. 
The response function $F_{1}, F_{2}, F_{3}$, and $F_{4}$ are defined above and refer to the primary and secondary side of the intermediate heat exchanger (IHX). The response function $F_{6}$ is defined in the same manner as $F_{1}$, except that the parameters for the primary and secondary sides of the air blast cooler $(A B C)$ are used in equation (14). The functions $F_{5}$ and $F_{7}$ are of the form $e^{-i \omega \tau}$ and represent pure delay time. The function $\mathrm{F}_{8}$ represents the delay between the core outlet and the inlet on the primary side of the IHX, as well as the coolant mixing that occurs between the core outlet and the vessel outlet, and is of the form $e^{-i \omega \tau} /(1+i \omega \lambda)$. The function $F_{9}$ corresponds to $F_{8}$, and represents similar processes between the outlet on the primary side of the IHX and the core inlet. The response function $F_{b}$ describes the frequency response of that portion of the coolant which bypasses the core and is of the form $\operatorname{Bexp}\left(-i \omega \tau_{c} / v_{r}\right)$ where $B$ is the fraction of coolant which bypasses the core, $\tau_{c}$ the core flow time at full flow and $v_{r}$ is the relative flow rate in the primary system. The function $\mathrm{F}_{\mathrm{nb}}=1-\mathrm{B}$. The functions $\overline{\mathrm{F}}$, $F_{p}, F_{i}$, and $F_{c}$ are obtained from equations (3) and (4) and are defined as follows:

$$
\begin{aligned}
& \bar{F}=\frac{\mathrm{K}_{\mathrm{N}}}{\mathrm{bv}}{ }_{\mathrm{r}}\left[\mathrm{a}_{\mathrm{c}} \mathrm{X}_{\mathrm{c}}+\left(1-\mathrm{a}_{\mathrm{c}}\right) \mathrm{F}_{\mathrm{s}} \mathrm{X}_{\mathrm{f}}\right] \int_{0}^{1} \mathrm{q}(\mathrm{z}) \mathrm{dz}[1-\exp \{-\mathrm{b}(1-\mathrm{z})\}] \\
& \mathrm{F}_{\mathrm{p}}=\frac{\mathrm{K}_{\mathrm{N}}}{\mathrm{v}_{\mathrm{r}}}\left[\mathrm{a}_{\mathrm{c}} \mathrm{X}_{\mathrm{c}}+\left(1-\mathrm{a}_{\mathrm{c}}\right) \mathrm{F}_{\mathrm{s}} \mathrm{X}_{\mathrm{f}}\right] \int_{0}^{1} \mathrm{q}(\mathrm{z}) \mathrm{dz} \exp \{-\mathrm{b}(1-\mathrm{z})\} \\
& \mathrm{F}_{\mathbf{i}}=\left(1-\mathrm{e}^{-\mathrm{b}}\right) / \mathrm{b} \\
& \mathrm{F}_{\mathrm{c}}=\mathrm{e}^{-\mathrm{b}}
\end{aligned}
$$


where the nomenclature of equations (3) and (4) are used.

The response of the average coolant temperature to a power oscillation at constant primary and secondary flow can be written as:

$$
\Delta \bar{T}=\left[\bar{F}+F_{i} F_{8} F_{9} R F_{n b} F_{p} /\left\{1-F_{8} F_{9} R\left(F_{b}+F_{n b} F_{c}\right)\right\}\right] \Delta P
$$

where $R$, the response of the primary outlet to a primary inlet oscillation is:

$$
R=F_{1}+F_{2} F_{3} F_{5} F_{6} F_{7} /\left(1-F_{4} F_{5} F_{6} F_{7}\right)
$$


GEAP -5576

APPENDIX IV

METHODS FOR DETERMING FREQUENCY COMPONENTS AND

EFFECTS OF FINITE DIGITIZING INTERVALS (ALIASING)

IV. 1 COMPARISON OF TWO METHODS

A method for eliminating the effects of drift (aperiodic terms) on the determination of the frequency components in an oscillator test has been described by Persson. (7) In this procedure, the detector signal is multiplied by an input signal (e.g. the reactivity osciliation) and the product is multiplied by an appropriate weighting function and integrated over successive half cycles. The weighting function is constant within a given half cycle and the ratio of the weighting function from the first half cycle to the last is given by the binomial coefficients. In the "conventional" method for determining the components, the weight function is constant and does not vary from half cycle to halt cycle.

Both of these methods were analyzed to determine how well they reject unwanted periodic signals which have a frequency at non-interger multiples of the input signal frequency. It was found that Persson's method has, in general, better rejection of unwanted periodic signals than the "conventional" method. In addition, the amplitude of noise with a constant frequency spectrum decreases as $2^{1-\mathrm{N}}$ for Persson's method, but only as $\mathrm{N}^{-1}$ for the conventional method, where $\mathrm{N}$ is the number of consective half cycles of data which have been treated. 
If the instrument signal, $f(t)$, is written in terms of its inverse Fourier transform as:

$$
f(t)=\int_{-\infty}^{\infty} A(\omega) \cos t \omega \cdot d \omega+\int_{-\infty}^{\infty} B(\omega) \sin \omega t \cdot d \omega
$$

The quantities which will be determined by the analysis are:

$$
F_{1}\left(\omega_{0}\right)=\frac{2 \omega_{0}}{\pi} \sum_{n=1}^{N} P_{n} \int_{(n-1)}^{n} \frac{\pi}{\omega_{0}} f(t) \cos \omega_{0} t d t
$$

and

$$
F_{2}\left(\omega_{0}\right)=\frac{2 \omega_{0}}{\pi} \sum_{n=1}^{N} \cdot P_{n} \int_{(n-1)}^{n} \frac{\pi}{\omega_{0}} f(t) \sin \omega_{0} t d t \cdots
$$

where the $P_{n}$ are defined by:

$$
\begin{aligned}
& \mathrm{P}_{\mathrm{n}}=\frac{1}{\mathrm{~N}} \quad \mathrm{n}=1,2, \ldots \mathrm{N} \text { (conventional method) }(\mathrm{N} \text { even) } \\
& \mathrm{P}_{\mathrm{n}}=\frac{\mathrm{q}_{\mathrm{n}}}{2^{\mathrm{N}-1}} \mathrm{n}=1,2, \ldots \mathrm{N} \text { (drift elimination method) }
\end{aligned}
$$

and the $q_{n}$ are the binomial coefficients (i.e. $q_{n}=\frac{(N-1) !}{(N-n) !(n-1) !}$

Interchanging the order of integration gives:

\section{Conventional Method}

$$
F_{1}^{\prime}=\int_{-\infty}^{\infty} \cdot d \omega[A(\omega)\{f(\omega)+f(-\omega)\}+B(\omega)\{-g(\omega)+g(-\omega)\}]
$$




$$
F_{2}=\int_{-\infty}^{\infty} d \omega[A(\omega)\{g(\omega)+g(-\omega)\}+B(\omega)\{f(\omega)-f(-\omega)\}]
$$

$$
\text { where: } \begin{aligned}
f(\omega) & =\frac{\sin \frac{N \pi}{\omega_{0}}\left(\omega_{0}-\omega\right)}{\frac{N \pi}{\omega_{0}}\left(\omega_{0}-\omega\right)} \\
g(\omega) & =\sin ^{2} \frac{N \pi}{2 \omega_{0}}\left(\omega_{0}-\omega\right) \\
\frac{N \pi}{2 \omega_{0}}\left(\omega_{0}-\omega\right) & \frac{\frac{N}{0}}{}
\end{aligned}
$$

\section{Drift Elimination Method}

$$
\begin{aligned}
& F_{1}=\int_{-\infty}^{\infty} d \omega[A(\omega)\{h(\omega)+h(-\omega)\}+B(\omega)\{-r(\omega)+r(-\omega)\}] \\
& F_{2}=\int_{-\infty}^{\infty} d \omega[A(\omega)\{r(11)+r(-\omega)\}+B(\omega)\{h(\omega)-h(-\omega)\}]
\end{aligned}
$$

where:

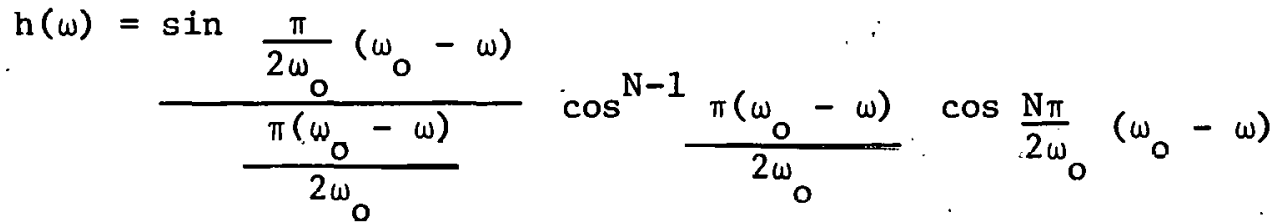

$$
\begin{aligned}
& r(\omega)=\sin \frac{\pi}{2 \omega}\left(\omega_{0}-\omega\right) \\
& \frac{2 \omega_{0} 0}{\frac{\pi\left(\omega_{0}-\omega\right)}{2 \omega_{0}}} \cos ^{N-1} \frac{\pi\left(\omega_{0}-\omega\right)}{2 \omega_{0}} \sin \frac{N \pi}{2 \omega_{0}}\left(\omega_{0}-\omega\right)
\end{aligned}
$$


Note that

$$
\begin{aligned}
\lim _{N \rightarrow \infty} f(\omega)=\lim _{N \rightarrow \infty} h(\omega)=f\left(\omega_{0}\right) & =h\left(\omega_{0}\right)=1 . \omega=\omega_{0} \\
& =0 \omega \neq \omega_{0} .
\end{aligned}
$$

and $\quad \lim _{N \rightarrow \infty} g(\omega)=\lim _{N \rightarrow \infty} r(\omega)=0$

Thus, in the limit, both the conventional and drift "filter" approach an ideal filter.

If $A(\omega)$ and $B(\omega)$ are constant, one can perform the indicated integration to obtain:

\section{Conventional Filter}

$$
F_{1}=A \frac{\omega_{0}}{N \pi}
$$

$$
\mathrm{F}_{2}=0
$$

\section{Drift Filter}

$$
\begin{aligned}
& F_{1}=A \frac{\omega_{0}}{\pi 2^{N-1}} \\
& F_{2}=0
\end{aligned}
$$

It is seen that the amplitude of noise with a constant frequency spectrum decreases faster (with increasing $N$ ) for the drift filter, than for the conventional filter.

$$
\text { IV- }
$$




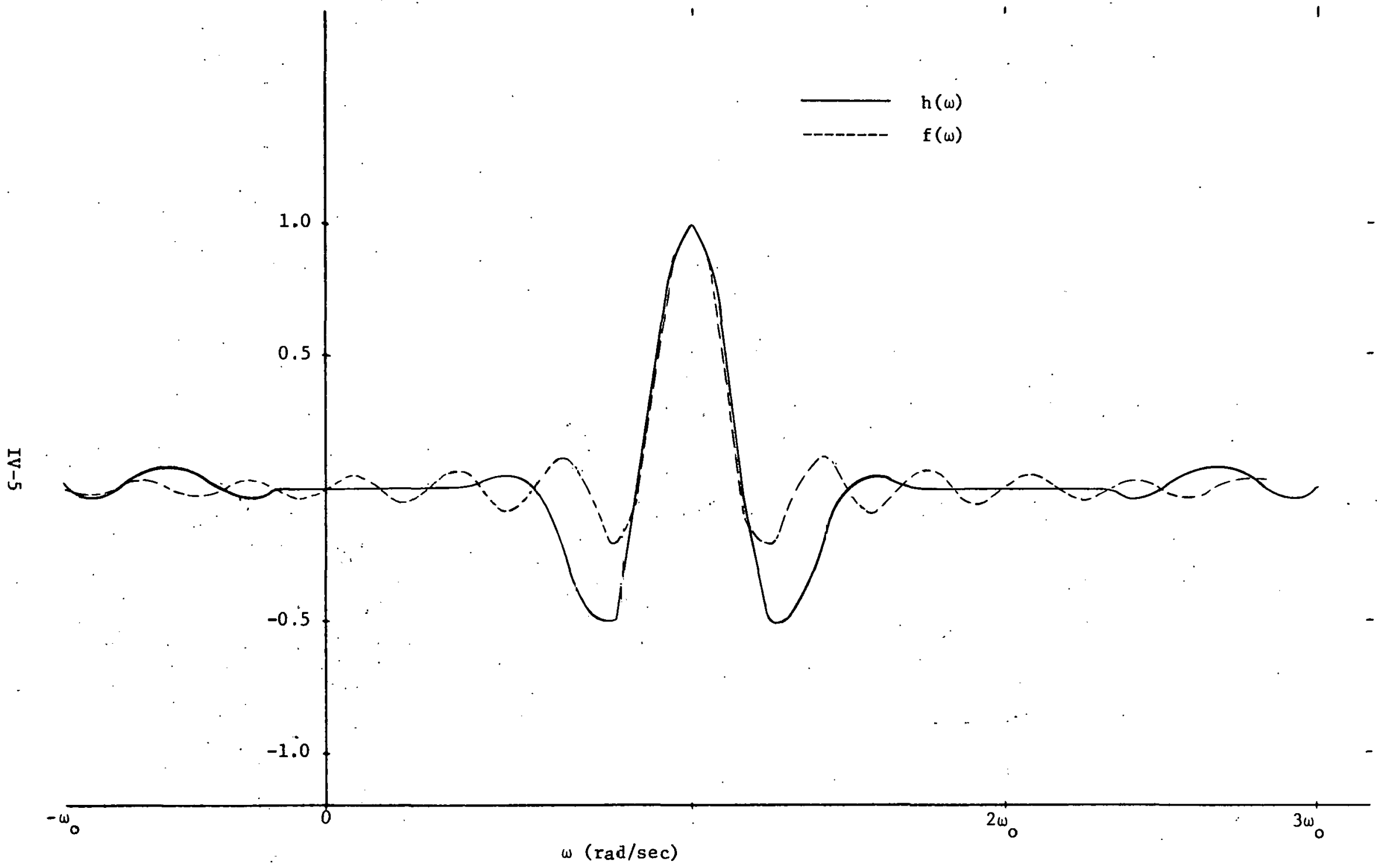

Figure IV -1 $\quad f(\omega)$ and $h(\omega)$ when $N=6$ 


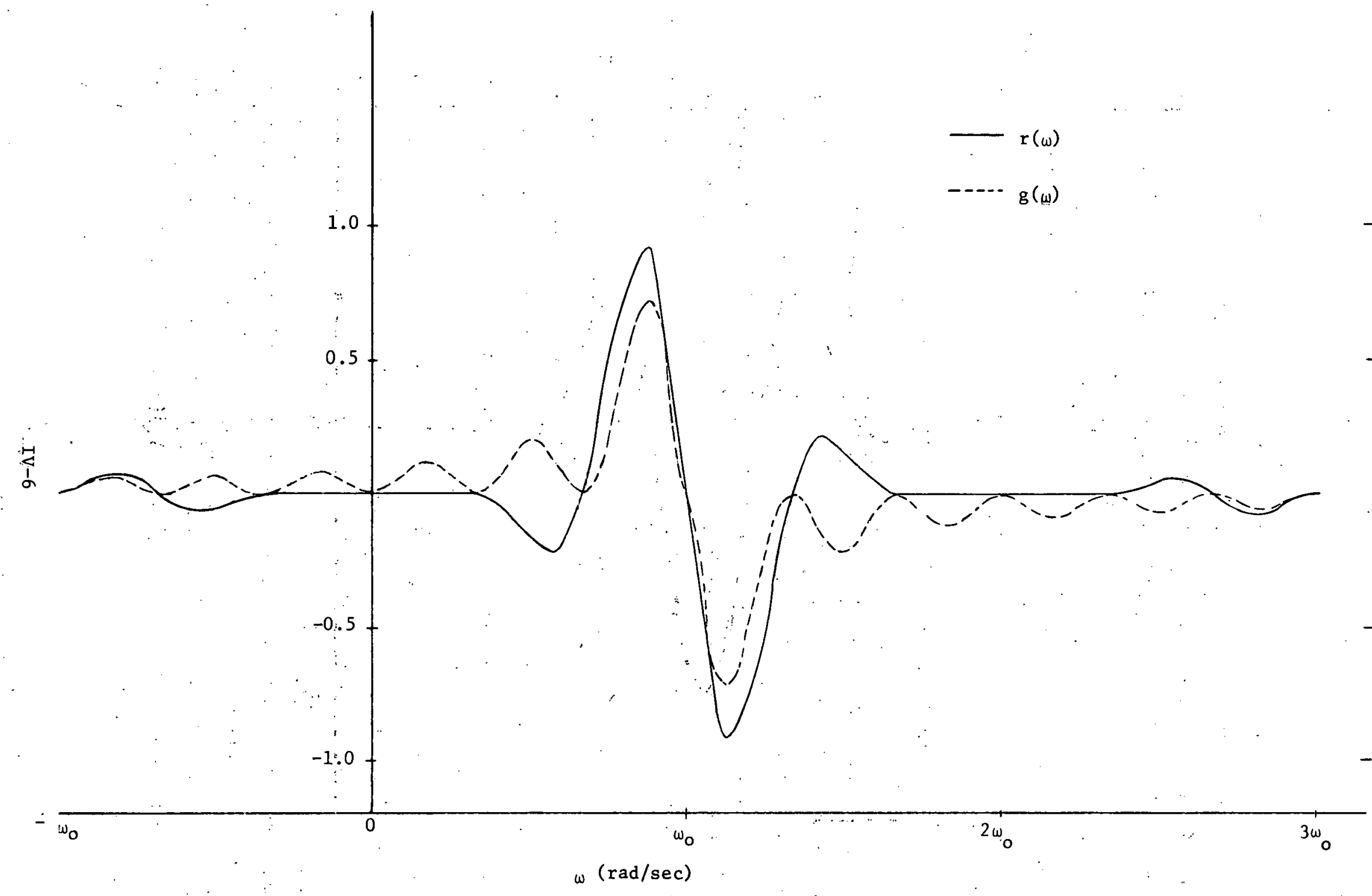

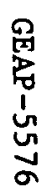

Figure IV-2 $\quad g(w)$ and $r(\omega)$ when $N=6$ 
If $f(t)$ can be presented by a $\left(\cos \omega_{0} t+b \sin \omega_{0} t\right)$, which will be the case for the "desired" component of the power, or temperature signal, $A(\omega)=a \delta\left(\omega-\omega_{0}\right)$ and $B(\omega)=b \delta\left(\omega-\omega_{0}\right)$. In this case $\mathrm{F}_{1}=\mathrm{a}$ and $\mathrm{F}_{2}=\mathrm{b}$ for both the conventional and drift filter.

Plots of the functions $f(\omega), h(\omega), g(\omega)$, and $r(\omega)$ are shown in Figures IV-1 and IV-2.

IV.2 EFFECTS OF FINITE DIGITIZING INTERVALS (ALIASING)

When a continuous signal is digitized at time intervals, $\Delta t$, any component of the signal which has a frequency greater than half the digitizing frequency $\left(f_{s}=1 / \Delta t\right)$ will appear, in the digitized data, as a component with frequency less than half the digitizing interval. This effect is commonly called aliasing $(8,9)$ and cannot be eliminated once the data is digitized. An example of this effect is shown below.

High frequency components can be eliminated from the sampled data. either by passing the continuous signal through an appropriate filter prior to sampling, or by sampling at a frequency which is at least twice the frequency of any undesired component in the signal. If it is assumed that all the high frequency components in SEFOR are part of the "noise", the low frequency noise spectrum (which is obtained by analyzing the signals with the same digitizing interval when the osclllator rod is oscillated at zero amplitude (see Section 5.0.3) w111 include the lifig frequency componenta, and the desired low frequency components of the signals can be obtained by subtracting the low frequency "noise". Note that if the sampling frequency that is used to sample the "noise" is different than the sampling frequency which is used when the desired signal is present, the effects of aliasing must be calculated. 
To provide an example of the effect of aliasing, it will be assumed that $B(\omega)$ in (1) is zero and only the "drift elimination". method described above will be considered. These assumptions do not alter the qualitative results obtained below: With these assumptions, the quantity $F_{1}$ of (6) can be written as:

$$
\begin{aligned}
F_{1} & =\frac{2 \omega_{0}}{\pi 2^{N-1}} \sum_{n=1}^{N} q_{n} \int_{(n-1)}^{\frac{n \pi}{\omega_{0}}} \frac{\pi}{\omega_{0}} f(t) \cos \omega_{0} t d t \\
& =\int_{-\infty}^{\infty} A(\omega) H(\omega) d \omega
\end{aligned}
$$

where

$$
H(\omega)=\frac{2 \omega_{0}}{\pi 2^{N}-1} \sum_{n=1}^{N} q_{n} \int_{(n-1)}^{\frac{n \pi}{\omega_{0}}} \frac{\pi}{\omega_{0}} \cos \omega t \cos \omega_{0} t d t
$$

Assume for simplicity that the signal $f(t)$ is digitized at time intervals $\Delta t=\pi / \omega_{0} K$ where $K$ is an integer. If the actual sampling does not produce. an integer number of samples per cycle, the necessary correction to the results obtained below will be very "small if the number of samples per. cycle is: large. "In addition to this assumption, let the integral in (9) be approximated by the trapezoidal rule (the exact method of numerical integration does not significantly affect the results): 
Rewriting (9) in terms of exponentials, using the relation:

$$
\sum_{k=1}^{K} e^{i k y}=e^{i(K+1) y / 2} \frac{\sin \frac{K y}{2}}{\sin y / 2}
$$

and noting that since the $q_{n}$ are the binomial coefficients the following relation applies:

$$
\begin{aligned}
\sum_{n=1}^{N} q_{n} e^{i 2 n y} & =e^{i 2 y}\left[1+e^{i 2 y}\right]^{N-1} \\
& =e^{i y(N+1)} 2^{N-1} \cos ^{N-1} y
\end{aligned}
$$

one obtains

$$
\begin{aligned}
& H(\omega)=S(\omega)+S(-\omega) \\
& S(\omega)=h(\omega) R(\omega)
\end{aligned}
$$

and where $h(\omega)$ is defined above.

The function $R(\omega)$ is defined by:

$$
R(\omega)=\frac{\Delta t}{2}\left(\omega_{0}-\omega\right) \cot \frac{\Delta t}{2}\left(\omega_{0}-\omega\right)=\frac{\pi}{2 \omega_{0} K}\left(\omega_{0}-\omega\right) \cot \frac{\pi}{2 \omega_{0} K}\left(\omega_{0}-\omega\right)
$$


$S(\omega)$ is a periodic function of $\left(\omega_{0}-\omega\right)$ with perriod equal to the sampling interval $\Delta t$, i.e. the frequency is equal to the sampling. frequency $f_{s}=1 / \Delta t$. If the sampling interval is much less than the integration time then $S(\omega)$, in the intervals

$$
f \equiv \frac{\omega}{2 \pi}=f_{0} \pm \frac{\ell f}{2} \quad(\ell=0,1,2, \ldots)
$$

looks very nearly the same as $h(\omega)$ in the interval $f=f_{0} \pm f_{s / 2}$. In the limit as $N$ (i.e. the integration time) $\rightarrow \infty, S(\omega)$ and $h(\omega)$ are identical in this interval, however $s(\omega)$, is periodic and $h(\omega)$ is not, since as $\mathrm{N} \rightarrow \infty, \mathrm{h}(\omega)$ approaches a Kronecker delta function at $\omega=\omega_{0}\left(f=f_{0}\right)$.

Since $S(\omega)=1$ when $\omega=\omega_{0}+2 \pi \ell f_{s}(l=0, \pm 1, \pm 2, \ldots)$ and $S(-\omega)=1$ when $\omega=\omega_{0}+2 \pi j f_{s}(j=0, \pm 1, \pm 2, \ldots)$,

$$
\begin{aligned}
& H\left(\omega_{o}\right)=1 \text { if } f_{s} / f_{o}>2 \\
& H\left(\omega_{0}\right)=2 \text { if } f_{s} / f_{o}=2 / \ell(\ell=1,2, \ldots)
\end{aligned}
$$

In other words, the fundamental component of the signal $\left(\omega=\omega_{0}\right)$ is obtained exactly, in the above approximation, if the number of samples/cycle $\left(\mathrm{f}_{\mathrm{s}} / \mathrm{f}_{\mathrm{o}}\right)$ is $>2$. If the number of samples/cycle is $<2$ erroneous results may be obțained.

In addition, it is seen that $H(\omega)=1$ when $\omega= \pm \omega_{0} \pm 2 \pi L f_{s}$ where $L$ takes on all integer values such that $\omega \geq 0$. For example, if 32 samples per cycle are recorded $f_{s}=16 \omega_{0} / \pi$ and all components of the signal with frequency $f>17 f$ appear in $H(\omega)$ and those frequency $f=f_{0}$, and $f=f_{0}(32 L \pm 1)$ are undamped. 
Thus for oscillations at $0.001 \mathrm{cycles} / \mathrm{sec}$, all frequencies $>.017$ cycles/sec appear in $H(\omega)$ and thus if $F_{1}\left(\omega_{0}\right)$ (i.e. in the measurement of the transfer function) and those components at 60.001 cps and 59.999 cps, in addition to many others, are undamped. 


\section{LEAST SQUARES CURVE FITTING}

\section{INTRODUCTION}

In this section, the general form of the "fitting" equation is presented. The derivation shown below is based on the derivation described by Deming ${ }^{(10)}$ and much of the notation is the same, however cases with "correlation" and with two "condition equations" are treated.

The purpose of the least squares fitting procedure is to determine values for the parameters in some mathematical model so that the sum of the weighted square of the residuals (the differences between measured "coordinates" and coordinates that are calculated by the model), is a minimum. As will be shown below, this is, in general, an iterative procedure, since initial estimates of the parameters are used to obtain new estimates, which can then be used as initial estimates for yet another iteration, and so on. If the initial estimates are reasonably close to the correct values, the procedure should converge quite rapidly.

\section{LEAST SQUARES CURVE FITTING}

It will be assumed in the following discussion that the measured values of the coordinates are randomly distributed about the "true or mean coordinates," and that the "mean" coordinates satisfy the condition equation when the true.values of the parameters are used in the mathematical model. See reference (11) for a discussion of the problem if the assumed "condition equation" is not the "true" equation. 
Let $x_{k n}$ denote the observed value of the $k-t h$ coordinate at point " $n$ " in the coordinate space, and let there be a total of $\mathrm{K}$ coordinates and a total of $\mathrm{N}$ points at which observations are made. The number $x_{k n}$ may perhaps be the mean of a number of measurements of coordinate " $k$ " at point " $n$ ", and each $x_{k n}$ may thus have an associated standard error $\sigma_{\mathrm{kn}}$. Since the measurements may be correlated, even at each point " $n$ ", there may also be coefficients of correlation, $\left(\mathrm{r}_{\mathbf{j k}}\right)_{n}$, between the $\mathrm{x}_{\mathbf{j} \mathrm{n}}$ and $\mathrm{x}_{\mathrm{kn}}$ coordinates:

Define the residual $\mathrm{v}_{\mathrm{kn}}$ as:

$$
v_{k n}=x_{k n} \text { (observed) }-x_{k n} \text { (calculated) }
$$

and define a set of $N$ column vectors, ${\underset{\sim}{n}}_{n}$, so that the $k$-th element of $\mathrm{V}_{\mathrm{n}}$ is $\mathrm{V}_{\mathrm{kn}}$.

The sum of the weighted squares of the residuals, $S$, can then be written as:

$$
S=\sum_{n=1}^{N} S_{n}=\sum_{n=1}^{N} \quad \underset{\sim n}{v} \bar{M}_{n} \underset{\sim n}{v}=\sum_{n=1}^{N} \sum_{k=1}^{K} \sum_{j=1}^{K} v_{k n}\left(M_{k n}\right)_{n} \cdot v_{j n}
$$

where $v_{\sim}^{T}$ 1s the transpose of ${\underset{\sim}{n}}_{n}$ and where $\bar{M}_{n}$ is a K $\times$ K symmetric matrix whose elements, $\left(M_{k j}\right)_{n}$, are the weights. The elements of the $\bar{M}_{n}$ matrices can be expressed in terms of the standard errors $\sigma_{k n}$ and the correlation: coefficients, $\left(r_{j k}\right)_{n} \cdot$ These elements will be discussed in more detail below.

The expression: for $S$ that is given above will first be rewritten in terms of the measured coordinates and the "true" pärameters, 'rather 
than measured coordinates and calculated coordinates, and the new expression for $\mathrm{S}$ will then be used to determine estimated values for these "true" parameters.*

In order to transform the expression for $S$, the following procedure, which is a slight modification of the derivation decribed by Deming (10) is used.

Let the calculated coordinates be subjected to $\mathrm{n}$ condition equations.

$$
\theta_{n}\left(x_{1 n}, x_{2 n}, \ldots . x_{K n}, a_{1}, a_{2}, \ldots a_{L}\right)=0 \quad(n=1,2, \ldots N)
$$

where the $a_{\ell}(l=1,2, \ldots . L)$ are the parameters, independent of $k$ and $n$, which are to be chosen so that $\underline{S}$ is a minimum and the condition equations are satisfied.

As an example of a condition equation: assume that coordinates $x_{1 n}$ and $x_{2 n}$ are the measured values at points $n=1,2, \ldots . N$ and that the "true coordinates" (i.e. the calculated coordinates for the true values of parameters, $a_{1}, a_{2}, a_{3}$ ) are $z_{1 n}$ and $z_{2 n}$. Further assume that $z_{1}=$ $a_{1}+a_{2} z_{2}+a_{3} z_{2}^{2}$. Then the condition equation can be written as:

$$
\theta_{n}=z_{1}-a_{1}-a_{2} z_{2 n}-a_{3} z_{2 n}^{2}=0 \quad(n=1,2, \ldots N)
$$

* It may be argued that the form of the transformed expression for $S$ (see equation 11) could be written down "a priori" when only one condition equation is used. The form of the transformed equation for $S$ when two condition equations are used was not so, "obvious", however, and the transformation described below were used to obtain the result given in equation (20). 
Neglecting higher order terms, the result obtained by expanding the condition equation $\theta_{n}$ in a Taylors series about the measured values of the coordinates, can be written as:

$$
\theta_{\mathrm{n}}=\mathrm{f}_{\mathrm{n}}-\mathrm{v}_{\mathrm{n}}^{\mathrm{T}} \quad \xi_{\mathrm{n}}=0
$$

where $f_{n}$ is $\theta_{n}$ evaluated at the measured values of the coordinates, and where $\xi_{\mathrm{n}}$ is a column vector with elements $\xi_{\mathrm{kn}}$ defined by:

$$
\xi_{k n}=\frac{\partial \theta_{n}}{\partial x_{k n} \text { (calculated) }}=\frac{\partial f_{n}}{\partial x_{k n} \text { (measured) }}
$$

where the partial derivatives are evaluated at the measured values of the coordinates.

For the example given above, $\xi_{1 n}=1$ and $\xi_{2 n}=-a_{2}-2 a_{3} x_{2 n}$, while $\mathrm{v}_{\mathrm{kn}}=\mathrm{x}_{\mathrm{kn}}-\mathrm{z}_{\mathrm{kn}}$ :

If $S$ is a minimum, the variation of $S$ is zero, and the variation $\delta S$ can be written (noting that $\bar{M}_{n}$ is symmetric) as:

$$
\frac{\delta S}{2}=\sum_{n=1}^{N} \cdot(\underset{\sim}{\delta})_{n}^{T} \cdot \bar{M}_{n} \underset{\sim n}{V}=0
$$

where the symbol $\delta$ denotes a variation and where $\underset{\sim}{\delta \mathrm{V}}$ is a column vector with elements $\delta V_{k n}$. 
Along with Deming, $(10)$ we note that the variations $\delta V_{k n}$ are not arbitrary, but must be chosen so that the condition equations are satisfied.

Taking the variation of (1), with the parameters $a_{\ell}$ fixed at their true values, and multiplying by an arbitrary non-zero scalar parameter $\lambda_{n}$; one obtains:

$$
\left.-\lambda_{\mathrm{n}} \underset{\sim}{(\delta \mathrm{V})}\right)_{\mathrm{n}}^{\mathrm{T}} \xi_{\mathrm{n}}=0
$$

The result of summing (3) over all $\mathrm{n}$ and adding to (2) is:

$$
\sum_{n=1}^{N}(\underbrace{V}_{n})_{n}^{T}\left(\bar{M}_{n} \underset{\sim n}{v}-\lambda_{n} \xi_{n}\right)=0
$$

There are $\mathrm{KN}$ variation in (4), but only $\mathrm{N}(\mathrm{K}-1)$ of these are independent because of (3). Huwever, the $\lambda_{n}$ are arbitrary and can be chosen so that $N$ of the coefficients of the variations in (4) vanish. Since the remaining $N(\mathrm{~K}-1)$ variations are independent, all coefficients in (4) must vanish. Thus:

$$
V_{n}=\lambda_{n} \bar{P}_{n} \xi_{n}
$$

where $\overline{\mathrm{P}}_{\mathrm{n}}$ is the Inverse of $\overline{\mathrm{M}}_{\mathrm{n}}$.

One finds, upon substitution of (5) into (1), that $\lambda_{n}=W_{n} F_{n}$ where the scalar $W_{n}$ is defined by:

$$
\frac{1}{W_{n}}=\xi_{n}^{T} \quad \bar{P}_{n} \xi_{n}
$$


Thus:

$$
{\underset{\sim}{\mathrm{n}}}_{\mathrm{n}}=\mathrm{w}_{\mathrm{n}} \mathrm{f}_{\mathrm{n}} \overline{\mathrm{P}}_{\mathrm{n}} \xi_{\sim_{\mathrm{n}}}=\mathrm{w}_{\mathrm{n}} \mathrm{f}_{\mathrm{n}}\left(\overline{\mathrm{M}}_{\mathrm{n}}\right)^{-1} \xi_{\mathrm{n}}
$$

and, finally, the sum of the weighted squares of the residuals becomes:

$$
s=\sum_{n=1}^{N} w_{n} f_{n}^{2}
$$

or, since $\theta_{\mathrm{n}}=0$

$$
s=\sum_{n=1}^{N} w_{n}\left(f_{n}-\theta_{n}\right)^{2}
$$

Thus, $S$ is the sum of the weighted squares of the deviations of an "observed", (if the true parameters $a_{\ell}$ were known) quantity $f_{n}$ about its true mean value $\theta_{n}$.

The weights, $W_{n}$, should be inversely proportional to the standard error in each observation $n$. Thus, if we define the elements $\left(P_{j k}\right)_{n}$ of $\overline{\mathrm{P}}_{\mathrm{n}}$, (the inverse of $\overline{\mathrm{M}}_{\mathrm{n}}$ ) by equation (9):

$$
\left(P_{j k}\right)_{n}=\frac{1}{\sigma_{0}^{2}}\left(r_{j k}\right)_{n} \sigma_{j n} \sigma_{k n}
$$

where $\sigma_{0}^{2}$ is an arbitrary constant, and where $\left(r_{j k}\right)_{n}$ is the coefficient of correlation between $\dot{x}_{j n}$ and $x_{k n}$, the weights $W_{n}$ are as follows:

$$
W_{n}=\frac{\sigma_{0}^{2}}{\sigma_{\text {fn }}^{2}}
$$


where the square of $\sigma_{f n}$, the standard eror in $f_{n}$, is given by:

$$
\begin{aligned}
\sigma_{f n}^{2} & =\sum_{j=1}^{K} \sum_{k=1}^{K} \xi_{j n} \xi_{k n}\left(r_{j k}\right)_{n} \sigma_{j n} \sigma_{k n} \\
& =\sigma_{0}^{2} \xi_{n}^{T} \bar{P}_{n} \xi_{n}
\end{aligned}
$$

where $r_{j j}=1$

Thus:

$$
s=\sum \frac{\sigma_{0}^{2}}{\sigma_{\mathrm{fn}}^{2}} \mathrm{f}_{\mathrm{n}}^{2}
$$

In the event that it is desired that the data simuitaneously satisfy two condition equations:

$$
\begin{aligned}
& \theta_{n}\left(x_{1 n}, x_{2 n}, \ldots . x_{K n}, a_{1}, a_{2}, \ldots a_{L}\right)=0 \\
& \phi_{n}\left(x_{1 n}, x_{2 n}, \ldots x_{K n}, a_{1}, a_{2} \ldots a_{L}\right)=0
\end{aligned}
$$

the above proredure can be modified in the following manner:

Expand $\phi_{n}$ in a Taylors series (neglecting higher order terms) to give:

$$
\phi_{\mathrm{n}}=\mathrm{g}_{\mathrm{n}}-{\underset{\sim}{\mathrm{n}}}_{\mathrm{v}}^{\mathrm{T}} \Psi_{\mathrm{n}}=0
$$


where $g_{n}$ is $\phi_{n}$ evaluated at the measured coordinates and the elements. $\psi_{\mathrm{kn}}$ of $\psi_{\mathrm{n}}$ are analogous to the $\xi_{\mathrm{kn}}$ of $\xi_{\mathrm{n}}$.

The variation of $\phi_{n}$ (multiplied by an arbitrary parameter $\nu_{n}$ ) is:

$$
-v_{n}(\underset{\sim}{V})_{n}^{T} \psi_{n}=0
$$

Summing (14) over all $\mathrm{n}$, adding to (4) and using the argument given following equation ( 4 ), one finds that:

$$
\underset{\sim n}{v}=\lambda_{n} \bar{P}_{n} \xi_{n}+\nu_{n} \bar{P}_{n} \Psi_{n}
$$

Using (15) in (1) and (13), one obtains the following:

$$
\lambda_{n}=\frac{\sigma_{0}^{2}}{\left[1-R_{n}^{2}\right]}\left[\frac{f_{n}}{\sigma_{f}^{2}}-\frac{R_{n} g_{n}}{\sigma_{f n} \sigma_{g n}}\right]
$$

and:

$$
\nu_{n}=\frac{\sigma_{0}^{2}}{\left[1-R_{n}^{2}\right]}\left[\frac{g_{n}}{\sigma_{g n}^{2}}-\frac{R_{n} f}{\sigma_{f n} \sigma_{g n}}\right]
$$


Thus, the calculated coordinate, $x_{k n}$ (calculated) $=x_{k n}$ (measured) - $\mathrm{V}_{\mathrm{kn}}$, can be written as:

$$
\begin{gathered}
x_{k n} \text { (calculated) }=x_{k n} \text { (measured) } \\
-\frac{1}{\left[1-R_{n}^{2}\right]} \sum_{j=1}^{K}\left(r_{j k}\right)_{n} \sigma_{j n} \sigma_{k n}\left\{\frac{f_{n} \xi_{j n}}{\sigma_{f n}^{2}}-\frac{R_{n}\left(\xi_{j n} g_{n}+\psi_{j n} f_{n}\right)}{\sigma_{f n} \sigma_{g n}}+\frac{g_{n} \psi_{j n}}{\sigma_{g n}^{2}}\right\}
\end{gathered}
$$

where $\sigma_{\text {fn }}^{2}$ is defined by $(10)$, where $\sigma_{\mathrm{gn}}^{2}$, the square of the standard error in $g_{n}$ is defined by (18):

$$
\sigma_{g n}^{2}=\sigma_{0}^{2} \quad \Psi_{n}^{T} \bar{P}_{n} \Psi_{n}
$$

where $R_{n}$, the coefficient of correlation between $f_{n}$ and $g_{n}$ is defined by (19).

$$
\mathrm{R}_{\mathrm{n}}=\frac{\sigma_{0}^{2}}{\sigma_{\mathrm{fn}}{ }^{\sigma} \mathrm{gn}} \quad \psi_{\mathrm{n}}^{\mathrm{T}} \quad \overline{\mathrm{P}}_{\mathrm{n}} \xi_{\mathrm{n}}=\frac{\sigma_{0}^{2}}{\sigma_{\mathrm{fn}}^{\sigma} \mathrm{gn}} \quad \xi_{\mathrm{n}}^{\mathrm{T}} \overline{\mathrm{P}}_{\mathrm{n}} \Psi_{\mathrm{n}}
$$

The sum of the weighted squares of the deviations is then given by $(20)$.

$$
\begin{aligned}
S & =\sum_{n=1}^{N} \frac{\sigma_{0}^{2}}{\left[1-R_{n}\right]}\left[\frac{f_{n}^{2}}{\sigma_{f n}^{2}}-\frac{2 R_{n} f_{n} g_{n}}{\sigma_{f n} \sigma_{g n}}+\frac{g_{n}^{2}}{\sigma_{g n}^{2}}\right] \\
& =\sum_{n=1}^{N} \frac{\sigma_{0}^{2}}{2}\left[\frac{\left(\frac{f_{n}}{\sigma_{f n}}-\frac{g_{n}}{\sigma_{g n}}\right)^{2}}{1-R_{n}}+\frac{\left(\frac{f_{n}}{\sigma_{f n}}+\frac{g_{n}}{\sigma_{g n}}\right)^{2}}{1+R_{n}}\right]
\end{aligned}
$$


The sum of the weighted squares of the residuals has thus been transformed to an expression (20) involving measured coordinates and the unknown parameters $a_{\ell}$.

The values of the parameters, $a_{\ell}$, which make $S$ in (20) a 'minimum can be found by an iterative procedure.' In order to retain linear equations for the iterations, the $f_{n}$ and $g_{n}$ in (20) are expanded in a Taylors series about estimated values $a_{\ell O}$, of the parameters $a_{\ell}$, and only the first two terms in the expansion are retained. Thus:

$$
f_{n} \simeq f_{n o}-\underset{\sim n}{F_{n}^{T}}\{\underset{\sim 0}{A}-\underset{\sim}{A}\}=f_{n o}-\underset{\sim}{F_{n}^{*}} \underset{\sim}{Y}
$$

and:

$$
g_{n} \simeq g_{n o}-G_{\sim n}^{T}\left\{A_{0}-A\right\}=g_{n o}-G_{n}^{T} Y
$$

where $\underset{\sim}{Y}={\underset{\sim}{0}}_{0}-\underset{\sim}{A}$. The elements $y_{\ell}$ of the vector $\underset{\sim}{Y}$ are equal to $a_{\ell 0} a_{\ell}$. The elements $F_{\ell n}$ of $\underset{\sim n}{F}$ and $G_{\ell n}$ of $\underset{\sim n}{G}$ are defined by:

$$
F_{\ell n}=\frac{f_{n}}{\partial a_{\ell}}
$$

and:

$$
G_{\ell n}=\frac{g_{n}}{\partial a_{\ell}} .
$$


where the partial derivatives are evaluated using the measured coordinates and the estimated values of the parameters. If the weights $\sigma_{0}^{2} / \sigma_{\mathrm{fn}}^{2}\left(1-\mathrm{R}_{\mathrm{n}}^{2}\right)$, etc. are evaluated using measured coordinates and estimated parameters, and if the partial derivatives of $S$ with respect to the $y_{\ell}$ are set equal to zero, the resultant equation for $\underset{\sim}{Y}$ can be written as:

$$
\overline{\mathrm{T}} \underset{\sim}{\mathrm{Y}}=\underset{\sim}{\mathrm{C}}
$$

where $\bar{T}$ is an LxL symmetric matrix with elements $T_{j k}$ defined by:

$T_{j k}=\sum_{n=1}^{N} \cdot \frac{\sigma_{0}^{2}}{\left[1-R_{n}^{2}\right]} \cdot\left[\frac{F_{j n} F_{k n}}{\sigma_{f n}^{2}}-\frac{2 k}{\sigma_{n n} \sigma_{g n}} F_{j n} G_{k n}+\frac{G_{j n} G k n}{\sigma_{g n}^{2}}\right]$

and where $\underset{C}{C}$ is a column vector with elements $C_{j}$ defined by:

$C_{j}=\sum_{n=1}^{N} \frac{\sigma_{0}^{2}}{\left[1-R_{n}^{2}\right]}\left[\frac{f_{n o} F_{n n}}{\sigma_{f n}^{2}}-\frac{R_{n}\left(f_{n o} G_{j n}+g_{n o} F_{j n}\right)}{\sigma_{f n} \sigma_{g n}}+\frac{g_{n o} G_{j n}}{\sigma_{g n}^{2}}\right]$

l'hus, $\overline{\mathrm{T}}$ and $\underset{\mathrm{C}}{\mathrm{C}}$ are known and $\underset{\sim}{\mathrm{Y}}$, as obtained from (24), is given by:

$$
\stackrel{\sim}{Y} \equiv(\overline{\mathrm{T}})^{-1} \underset{\sim}{\mathrm{C}}=\overline{\mathrm{Q}} \underline{\mathrm{C}}
$$

where $\bar{Q}=(\bar{T})^{-1}$. 
The new estimates of the parameters $a_{\ell}$ are obtained from (27).

$$
\underset{\sim}{A}=\underset{\sim 0}{A}-\underset{\sim}{Y}=\underset{\sim 0}{A}-\bar{Q} \underset{\sim}{C}
$$

and the value of $\mathrm{S}$ can be calculated, once $\underset{\sim}{\mathrm{Y}}$ is known, from equations (20), (21), and (22).

The square of the estimated standard error $\sigma_{\ell}$ in $a_{\ell}$ is given by (28)

$$
\sigma_{\ell}^{2}=Q_{\ell \ell} \sigma^{2}
$$

where the $Q_{\ell \ell}$ are diagonal elements of $\bar{Q}$ and where $\sigma^{2}=\frac{S}{\mu}$, where $\mu$ is the number of degrees of freedom (i.e. the number of data points $N$ times the number of condition equations ( 1 or 2 ) minus the number of parameters L).

The off-diagonal elements of the $\bar{Q}$ matrix are related to the coefficients of correlation between parameters $a_{j}$ and parameter $a_{k}$. In general, (since $r_{j j}=1$ ),

$$
\sigma_{j} \sigma_{k} r_{j k}=Q_{j k} \sigma^{2}=Q_{j k} \frac{S}{\mu}
$$

The standard error $\sigma_{z}$ in a function $z=z\left(x_{1}, x_{2}, \ldots x_{K}, a_{1}, a_{2}, \ldots a_{L}\right)$ is thus given by:

$$
\sigma_{z}^{2}=\sigma^{2} \sum_{j=1}^{L} \sum_{k=1}^{L} \frac{\partial Z}{\partial a_{j}} \frac{\partial Z}{\partial a_{k}} \cdot Q_{j k}
$$


In the particular case that the condition equation $\theta_{n}$ is of the form:

$$
\theta_{n}=z_{n}-z_{n}\left(x_{1 n}, x_{2 n}, \ldots x_{K n}, a_{1}, a_{2} \ldots a_{L}\right)=0
$$

the standard error in the calculated curve for $z$ at point $n$ is obtained from:

$$
\sigma_{z n}^{2}=\sigma^{2} \quad \sum_{j=1}^{L} \sum_{k=1}^{L} F_{j n} F_{k n} Q_{j k}
$$

Error bands, $z_{n} \pm \sigma_{z n}$, can be plotted to show the standard error in $z$ above and below the calculated curve (see Deming ${ }^{(10)} \mathrm{pg} \cdot 228$ for example). A confidence band is obtained if $\sigma$ is replaced by $\sigma \mathrm{T}$, when " $T$ " is obtained from students " $t$ " distribution. The confidence band, rather than the error band, should be used if the number of degreas of freedom is small.

If one of the $a_{\ell}$ (say $a_{m}$ ) is changed by an amount $\Delta a_{m}$, is held constant at the new value, and the quantity $S$ is minimized with respect to the other $a_{\ell}(\ell \neq m)$, the new values will differ from the old values by an amount $\Delta a_{\ell}$ which is approximately given by the following:

$$
\Delta a_{\ell}=\frac{Q_{\ell m}}{Q_{m m}} \Delta a_{m}
$$

The corresponding change in $\mathrm{S}$ is approximately given by:

$$
\Delta S=\left(\Delta a_{m}\right)^{2} / Q_{m m}
$$

Explict examples of condition equalions for the analysis of oscillator, static, and transient data are given in the text of this report. 
Estimated errors in the real and imaginary parts of the reactivity feedback for a conventional oscillator test were obtained by using the calculated SEFOR transfer function (e.g. Section 5.2.4). It was assumed that the measured quantities are $G$, the at-power transfer function; $G_{0}$, the zero power transfer function; $\Delta \mathrm{T}_{c}$, the average coolant temperature oscillation; and $\Delta \mathrm{P} / \mathrm{P}_{\mathrm{O}}$, the fractional power oscillation.

Using the notation of Section 2.5, the product of the power level and the transform of the feedback kernel can be written as:

$$
\mathrm{H} \equiv \mathrm{Ph}=\left(\frac{1}{G_{0}}-\frac{1}{G}\right)=\alpha_{f} F_{a v} P X_{f}+\left[C_{f} F_{s}+C_{q}\right] \frac{\Delta T_{C}}{(\Delta P / P)}
$$

where the reactivity e[fecl lue to inlet tempcrature oecillations has been neglected.

The inverse, $\sigma_{H}^{2}$, of the weighting functions for a least squares fitting of the above model to the data (i.e. the "effective standard error") is calculated from:

$$
\sigma_{\mathrm{H}}^{2}=\left[\frac{\sigma_{\mathrm{Go}}^{2}}{\left|G_{\mathrm{O}}\right|^{4}}+\frac{\sigma_{G}^{2}}{|G|^{4}}\right]+\left|\mathrm{C}_{\mathrm{S}} \mathrm{F}_{\mathrm{S}}+\mathrm{C}_{\mathrm{q}}\right|^{2}\left[\frac{\sigma_{\mathrm{T}}^{2}}{\left|\frac{\Delta \mathrm{P}}{\mathrm{P}}\right|^{2}}+\left|\frac{\Delta \mathrm{T}}{\Delta \mathrm{P} / \mathrm{P}}\right|^{2} \frac{\sigma_{\mathrm{P}}^{2}}{\left|\frac{\Delta \mathrm{P}}{\mathrm{P}}\right|^{2}}\right]
$$


where it has been assumed that:

$$
\begin{aligned}
\sigma_{\mathrm{Go}} & =\text { standard error in real part of } \mathrm{G}_{\mathrm{O}} \\
& =\text { standard error in imaginary part of } \mathrm{G}_{\mathrm{O}} \\
\sigma_{\mathrm{G}} & =\text { standard error in real part of } \mathrm{G} \\
& =\text { standard error in imaginary part of } \mathrm{G} \\
\sigma_{\mathrm{T}} & =\text { standard error in real part of } \Delta \mathrm{T}_{\mathrm{C}} \\
& =\text { standard error in imaginary part of } \Delta \mathrm{T} \\
\sigma_{\mathrm{P}} & =\text { standard error in real part of } \Delta \mathrm{P} / \mathrm{P} \\
& =\text { standard error in imaginary part of } \Delta \mathrm{P} / \mathrm{P}
\end{aligned}
$$

Thus, $\sigma_{\mathrm{H}}^{2}$ is the inverse of the weighting function for a fit of both the real and imaginary parts of equation (1) to the data.

The calculated percent deviation in the real and imaginary parts of $H\left(\left|\sigma_{H} / R_{H}\right|\right.$ and $\left.\left|\sigma_{H} / I_{H}\right|\right)$ are shown in Figures VI-1 and VI-2, for the following set of assumptions: It was assumed that the error $\left|\sigma_{\text {Go }} / G_{0}\right|$ in the zero power transfer function, $\left|\sigma_{G} / G\right|$ in the at-power transfer function and, $\left|\sigma_{\mathrm{P}} / \Delta \mathrm{P} / \mathrm{P}\right|$ in the fractional power oscillation were all equal to $1.0 \%$. The assumed error for $\sigma_{T}$ was $\pm 0.125^{\circ} \mathrm{F}$ (this is $a$ minimum error, since it represents the estimated resolution of the data acquisition system, but does not include random, or othér, errors in thermocouple signals). A $5 \%$ power oscillation $\Delta P / P$ was also assumed.

At low frequencies, the contribution of the error, $\sigma_{\mathrm{T}}^{2}$, in $\Delta \mathrm{T}_{\mathrm{c}}$ to the total squared error $\sigma_{\mathrm{H}}^{2}$ is $\sim 85 \%$ at $5 \mathrm{MW}$ and $\sim 50 \%$ at $20 \mathrm{MW}$. Since the squared error $\sigma_{\mathrm{H}}^{2}$ contains the term $\sigma_{\mathrm{T}}^{2} /|\Delta \mathrm{P} / \mathrm{P}|^{2}$, the error in $\sigma_{\mathrm{H}}$ can be reduced, other things remaining constant, by increasing the fractional. power oscillation from $5 \%$ to $10 \%$. 
At higher frequencies (approching $0.1 \mathrm{cps}$ ), the percent error in $\sigma_{H}$ becomes more dependent on the errors in $G$ and $G_{0}$ and less dependent on the error in the coolant oscillation and the fractional power oscillation (i.e., $G_{0} \rightarrow G$ and $h \rightarrow 0$ as $\omega \rightarrow \infty$ ). 


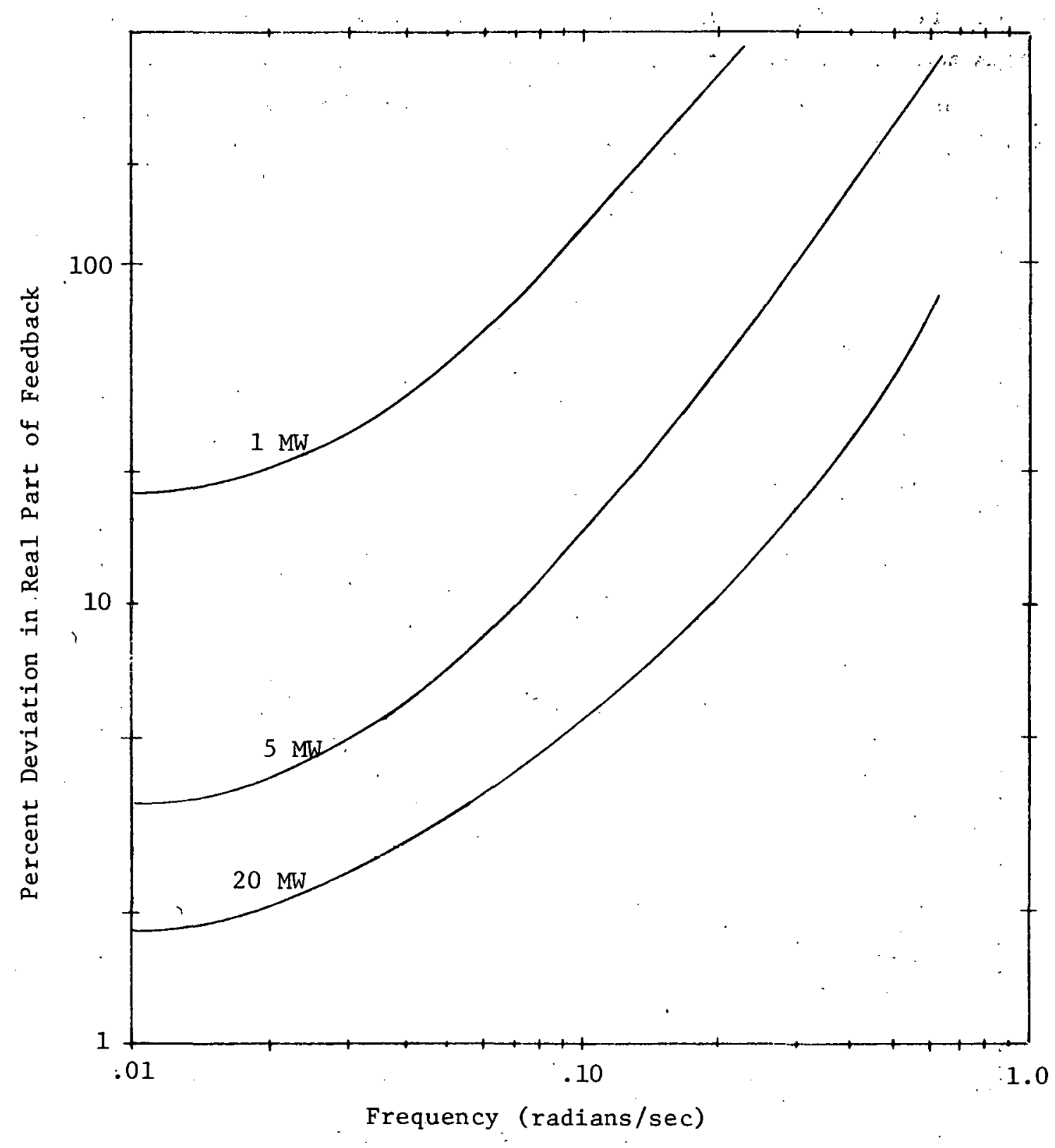

Figure VI-1. ESTIMATED DEVIATION IN REAL PART OF THE FEEDBACK (CONVENTIONAL OSCILLATOR TESTS) 


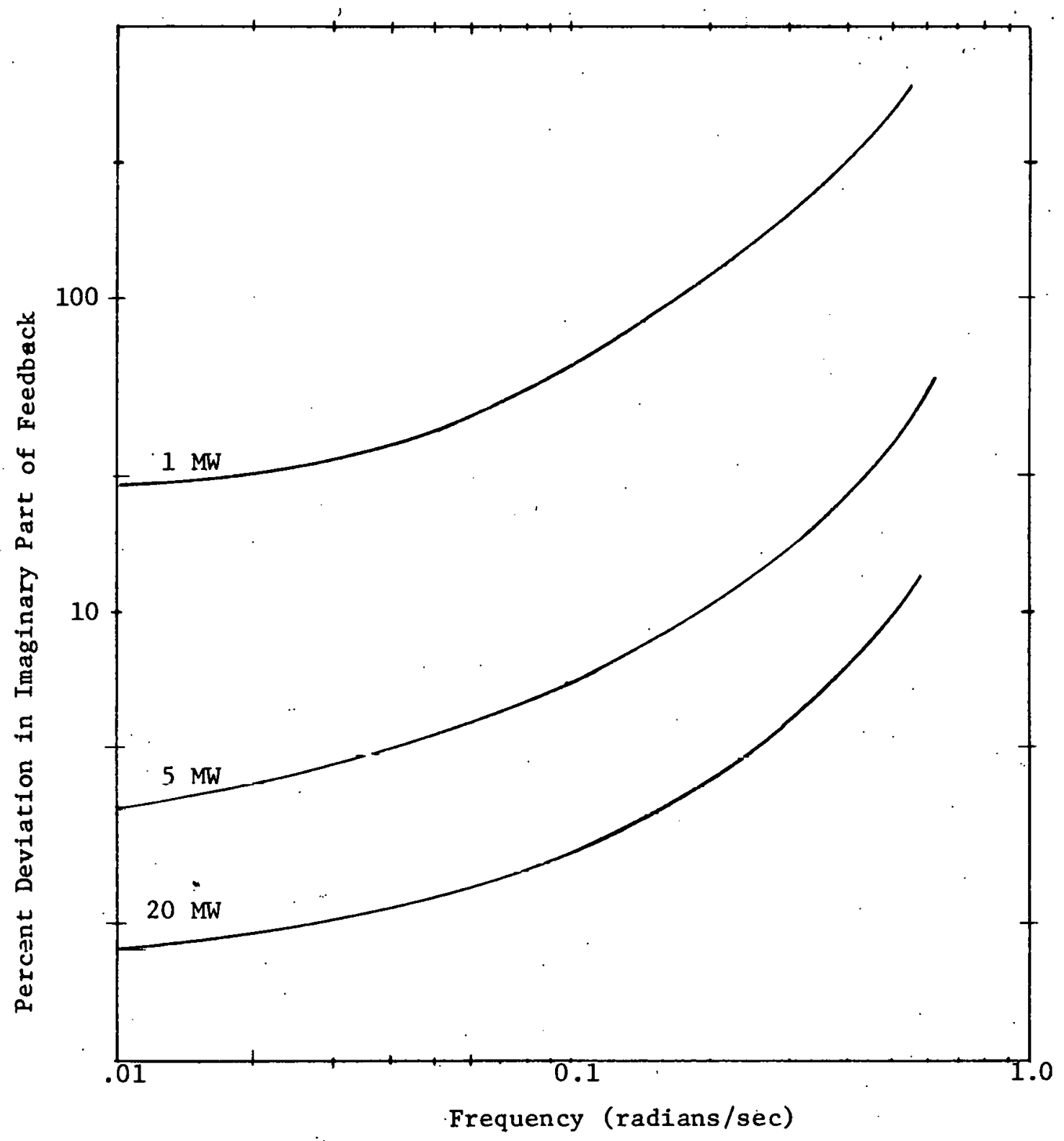

Figure VI-2. ESTIMATED DEVIATION IN IMAGINARY PART OF THE FEEDBACK (CONVENTIONAL OSCILLATOR TESTS) 
TIME DEPENDENT REACTIVITY WORTH OF A REFLECTOR AND

THE FAST REACTIVITY EXCURSION DEVICE (FRED)

Calculated curves for the approximate time dependent behavior of reflector motion and of the FRED are shown below. Figure VII-1 illustrates the estimated ${ }^{(12)}$ reflector position as a function of time during the first portion of its travel. Figure VII-2 approximates the reflector reactivity-position curve for a two-segment core as one $\$ 1.3$ reflector is lowered from the completely up position; and was obtained from a renormalization of the data in reference (13). The actual curve for a given SEFOR reflector may be somewhat different because of "shadowing" effects of the reflectors and because (1) in the ZPR-3 mockup of SEFOR, the reflector was removed from the bottom rather than the top, and (2) in the ZPR-3 experiment, the reflector extended one-inch above the core while in SEFOR, the reflector will extend u-inches above the core.

Let the FRED slug length (12-inches) be denoted by $L$, the extrapolated core half height by $\mathrm{H}$, and the distance that the center of the slug is displaced above the core center by $z$. The FRED worth at any position $z$ is given by perturbation theory as:

$$
\begin{array}{ll}
k(z)=\int_{z-L / 2}^{z+L / 2} \phi^{2}(x) d x & z+L / 2 \leq H \\
k(z)=\int_{z-L / 2}^{H} \phi^{2}(x) d x & H-L / 2 \leq z \leq H+L / 2 \\
k(z)=0 & a-L / 2 \geq H
\end{array}
$$


where $\phi^{2}(z)$ is the worth of a small sample at location $z$.

For the two-segment core $\phi^{2}(z)$ was obtained from a fit of Figure 13 of reference (13). The $\mathrm{Pu}$ axial reactivity traverse was used since no boron traverse was available for the two-segment core. The fit is given below and the normalized reactivity is shown in Figure VII-4.

$$
\begin{array}{lr}
\phi^{2}(z)=\sin ^{2} \cdot \frac{\pi}{2}\left(\frac{25+z}{22}\right) & -25 \leq z \leq 5 \\
\phi^{2}(z)=.638+.035(7-z) & 5 \leq z \leq 7 \\
\phi^{2}(z)=.668 \sin ^{2} \frac{\pi}{2}\left(\frac{26-z}{22}\right) & 7 \leq z \leq 26
\end{array}
$$

The estimated ${ }^{(14)}$ FRED displacement as a function of the time shown in Figure VII-5 was used to: generate the FRED reactivity vs time curve shown in Figure VII-6 and VII-7. 


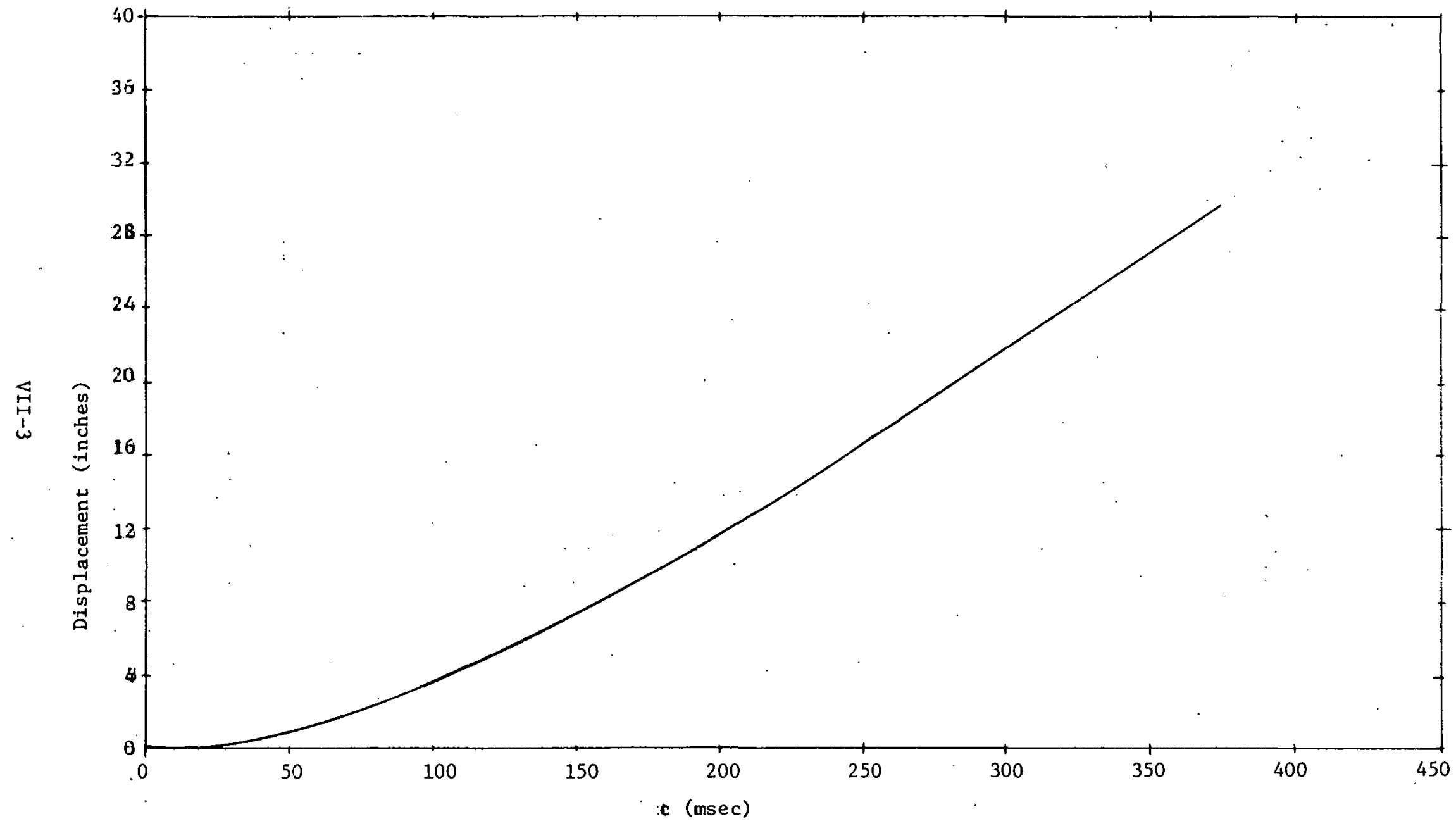

Figure VII-1. ESTIMATED REFLECTOR POSITION VS TIME 
GEAP -5576

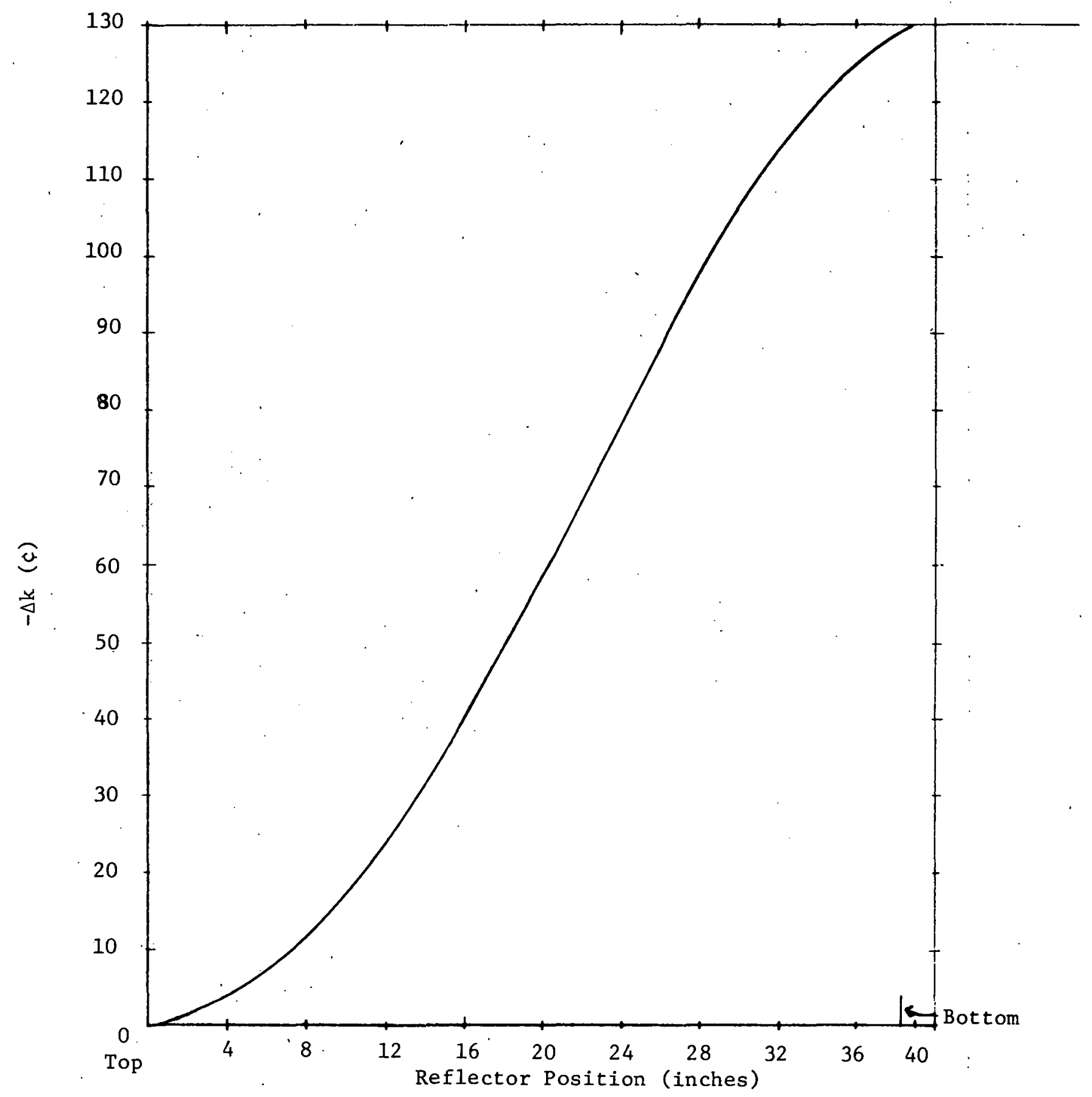

Figure VII-2. REFLECTOR WORTH VS POSITION FOR ", 2-SEGGENT CORE 


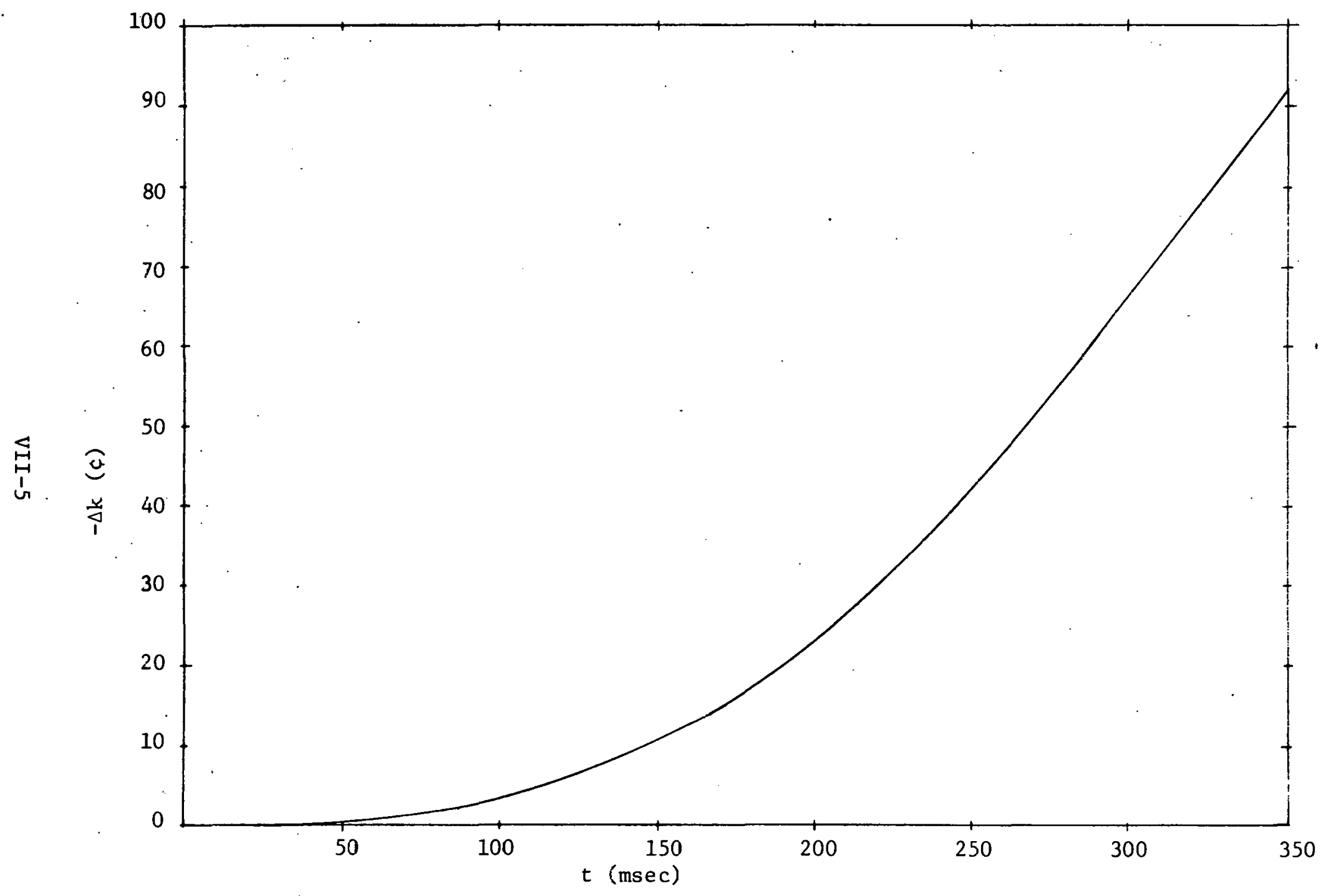

Figure VII-3. ESTIMATED RELFECTOR WORTH ( $\$ 1.3$ TOTAL) AS A

FIINCTION OF TIME 


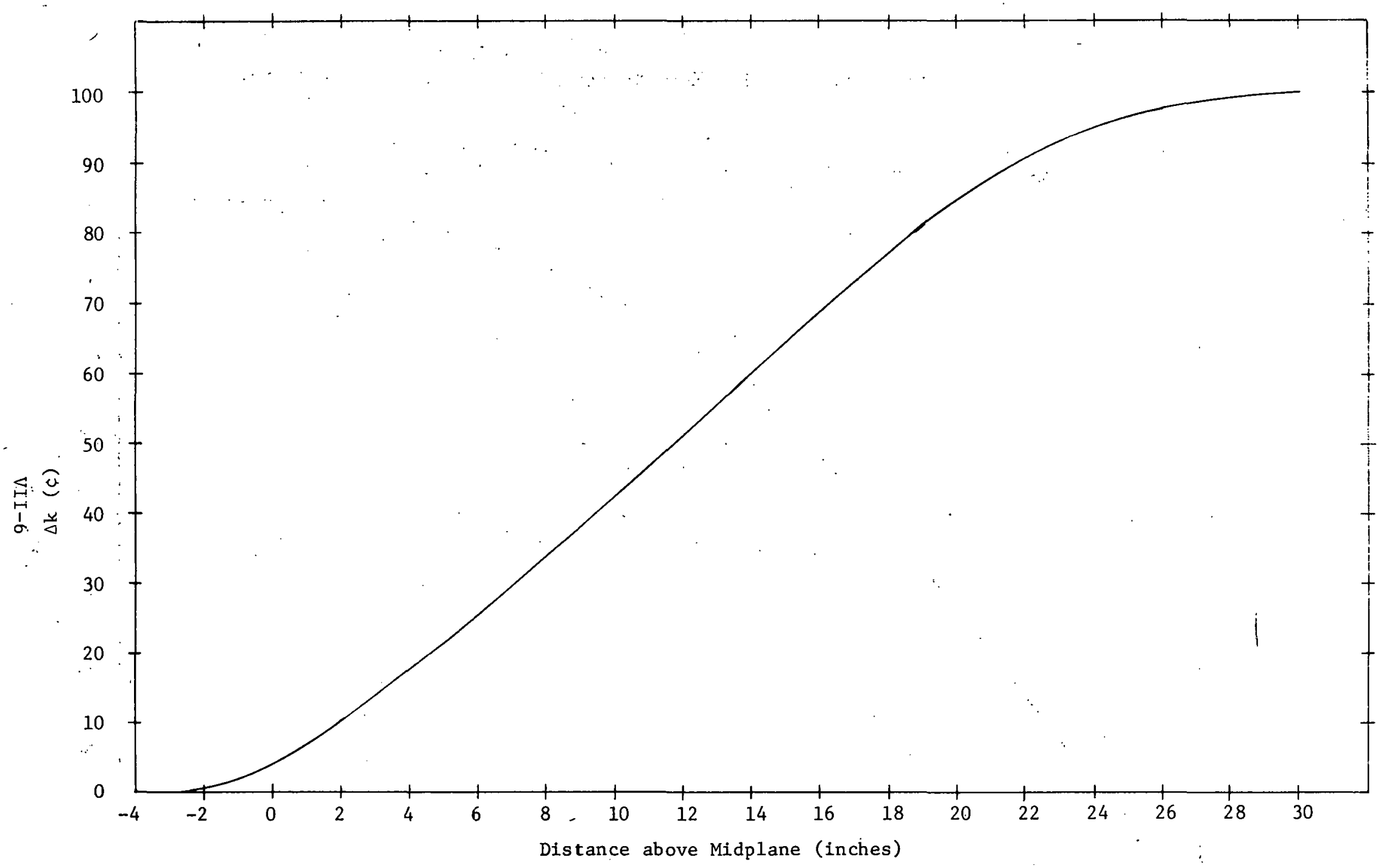




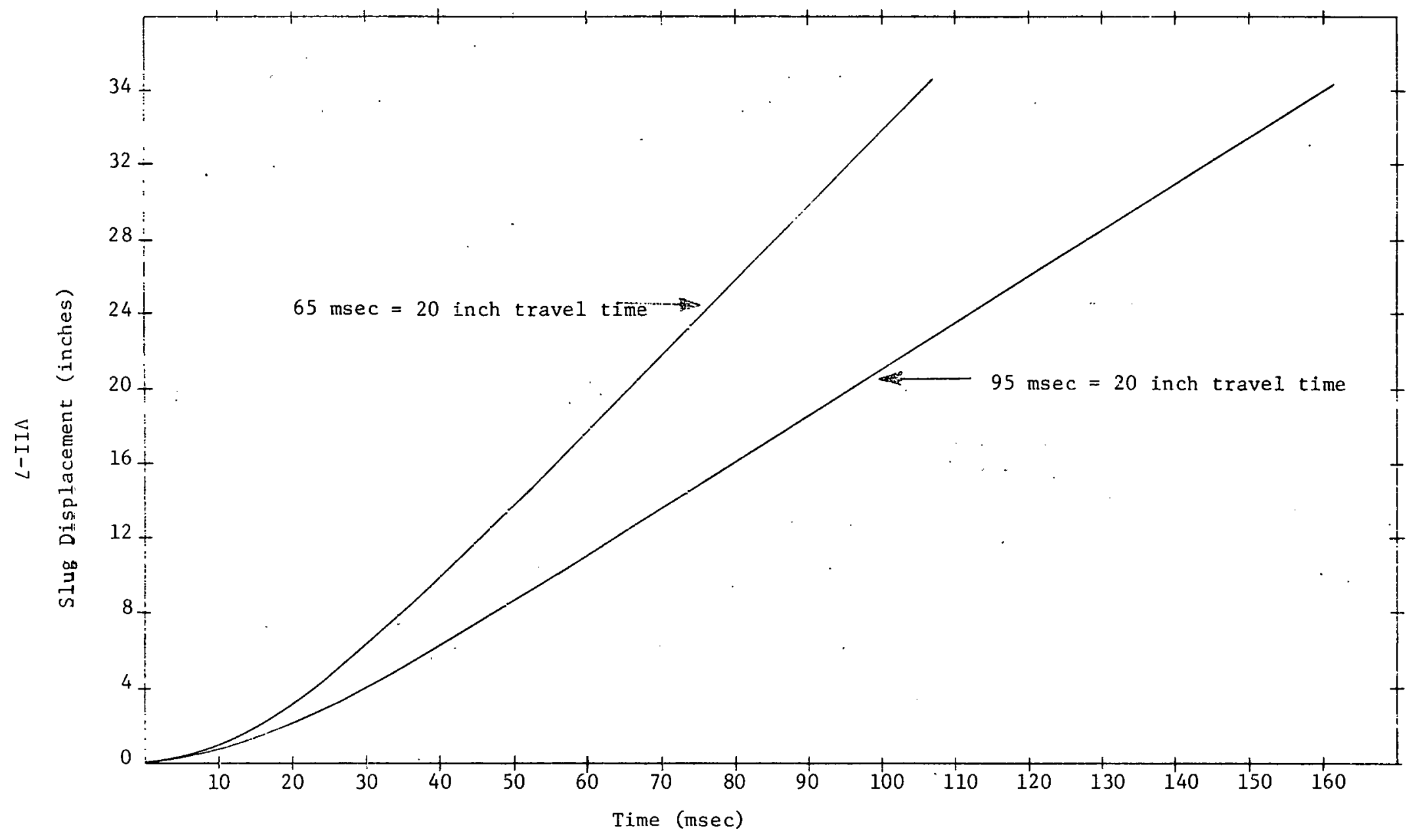

Figure VII-5. $\quad$ FRED DISPLACEMENT VS. TIME 


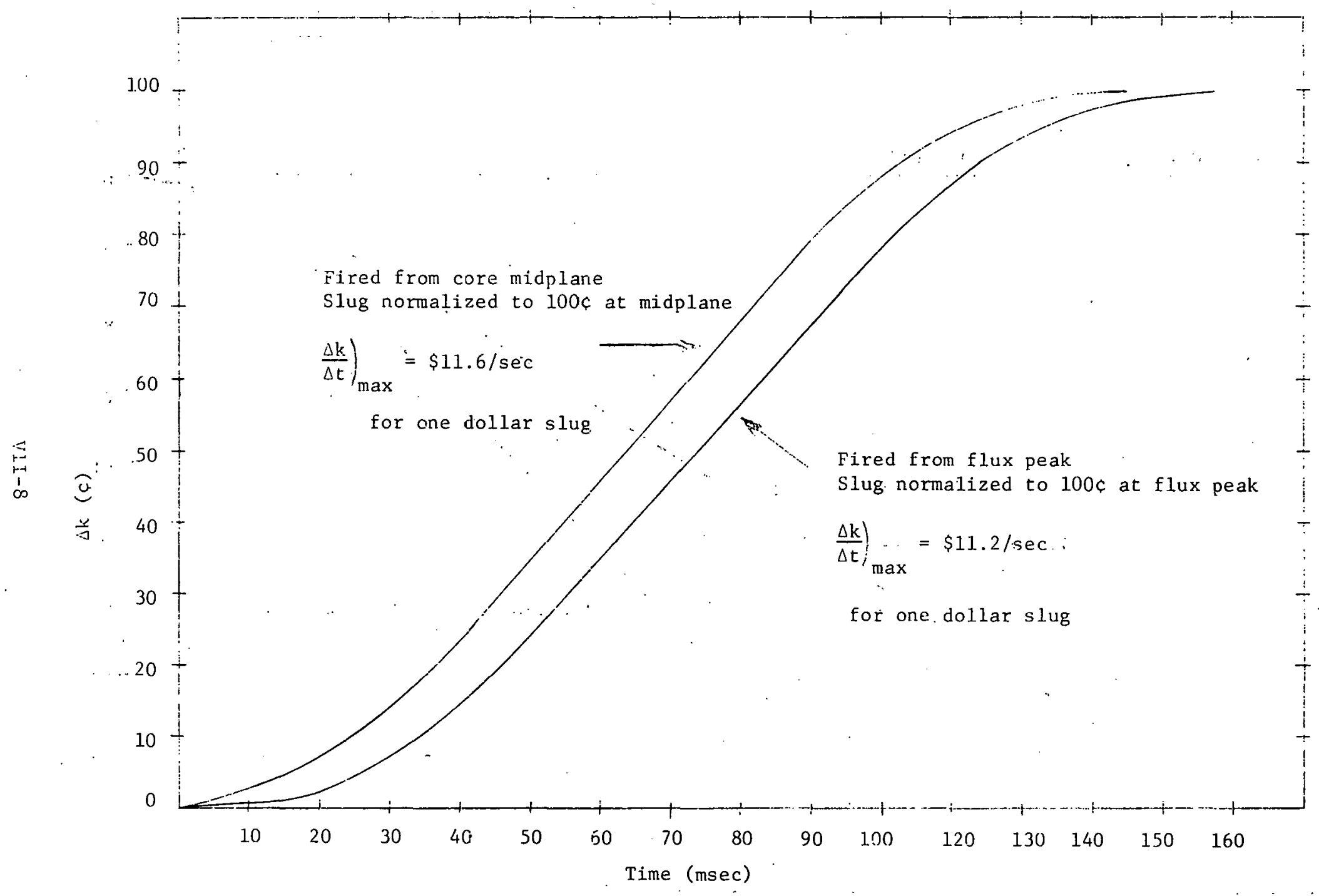

Figure VII-6. TIME DEPENDENT FRED WORTH FOR 20-INCH

TRAVEL TIME OF 95 MSEC 


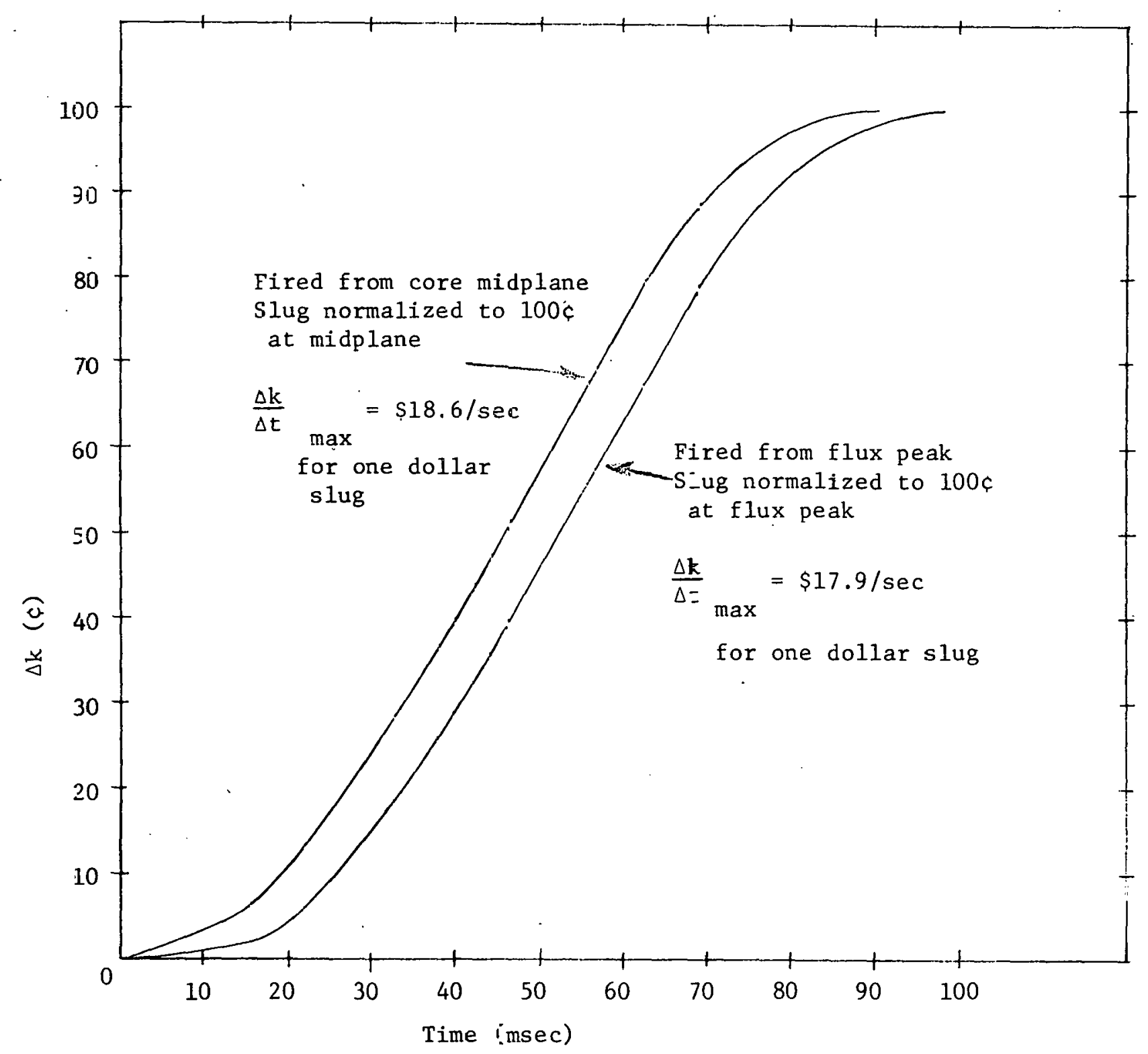

Figure VII-7. TIME DEPENDENT FRED WORTH FOR 20-INCH TRAVEL TIME OF 65 MSEC 
GEAP -55.76

APPENDIX VIII

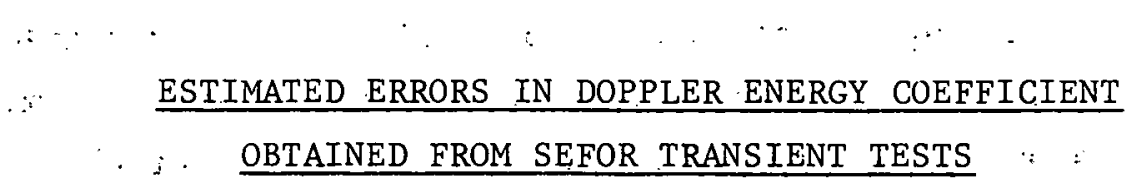

Estimates were made of the expected errors:in the Doppler energy coefficient for transient experiments in SEFOR, where: the silug is. completely.: ejected in $\sim 90 \mathrm{msec}$.. The errors. were calculated for various. FRED slug sizes with and without reflector motion prior to FRED ejection: The FORE ${ }^{(15)}$ code was. used to generated the feedback: effects during. the.. . various transients, and the errors in the Doppler energy coefficient were calculated by assigning assumed uncertainties to the FRED worth, fuel expansion effect, etc.

The percent error in the Doppler energy coefficient. is shown in Figure VIII-1 as a function of FRED; slug size (with and without reflectior motion prior to: FRED ejection) for two different assumptions (see below). about the magnitude of the uncertainties in the various reactivity effects. Although the actual numbers may change as a'result of a more thorough analysis, two trends are indicated by Figure VIII-1. These are: (1) the error is very large for FRED slü sizes $\lesssim \$ 1.3$ when reflector motion is initiated prior to FRED ejection, and (2) little increase in accuracy is obtained by using slug sizes $\gtrsim \$ 1.3$ when there is no reflector motion.

Using the assumptions described below, the values of the various reactivity effects were taken from several. FORE runs and the error in the Doppler energy coefficient was calculated at various time intervals during a transient. The calculated error reached a minimum'during the first one or two hundred milliseconds after FRED had been completely ejected and did not vary greatly during this time interval. An average 
value of the error during a time interval of $\approx 100 \mathrm{msec}$ after complete FRED ejection is shown as a calculated point in Figure VIII-1. Some explicit examples of the calculations are shown at the end of this Appendix.

The primary reason that the error in the Doppler energy coefficient is so large with reflector motion and slug sizes $\lesssim \$ 1.3$ is not because the magnitude: of the errors in FRED, etc., change significantly, but rather that the magnitude of the Doppler feedback itself is decreased from that of the case with no reflector motion. As an example: in the case of a $\$ 1.1$ FRED slug and no reflector motion, the Doppler $10 \mathrm{msec}$ 'after 'complete FRED ejection is $14.5 \mathrm{c}$ ' while the corresponding Doppler feedback in the case of reflector motion is only 4.3c. For slug sizes $z \$ 1.3$ the FRED overrides the reflector and the Doppler feedback approaches the no-reflector-motion case. The effect of reflector motion upon the power, and thus the fuel temperature and Doppler effect, is illustrated in Figure VIII-2 for a $\$ 1.10$ FRED slug:

\section{VIII.1 REACTIVITY EFFECTS}

All of the results presented here are based upon the case in which. the reactor is initially at $5 \mathrm{MW}$ and the system has the following reactivity coefficients.

$$
\begin{aligned}
& \text { Doppler } \mathrm{T} \frac{\mathrm{dk}}{\mathrm{dT}}=-.008 \equiv \mathrm{A} \\
& \text { Fuel axial expansion }=-1.32 \times 10^{-6} \Delta \mathrm{k} /{ }^{\circ} \mathrm{F} \equiv \alpha_{\mathrm{e}} \\
& \text { Clad expansion }=-2.44 \times 10^{-6} \Delta \mathrm{k} /{ }^{\circ} \mathrm{F} \equiv \alpha_{\mathrm{C}} \\
& \text { Structure and sodium }=-5.0 \times 10^{-6} \Delta \mathrm{k} /{ }^{\circ} \mathrm{F} \equiv \alpha_{\mathrm{s}}
\end{aligned}
$$




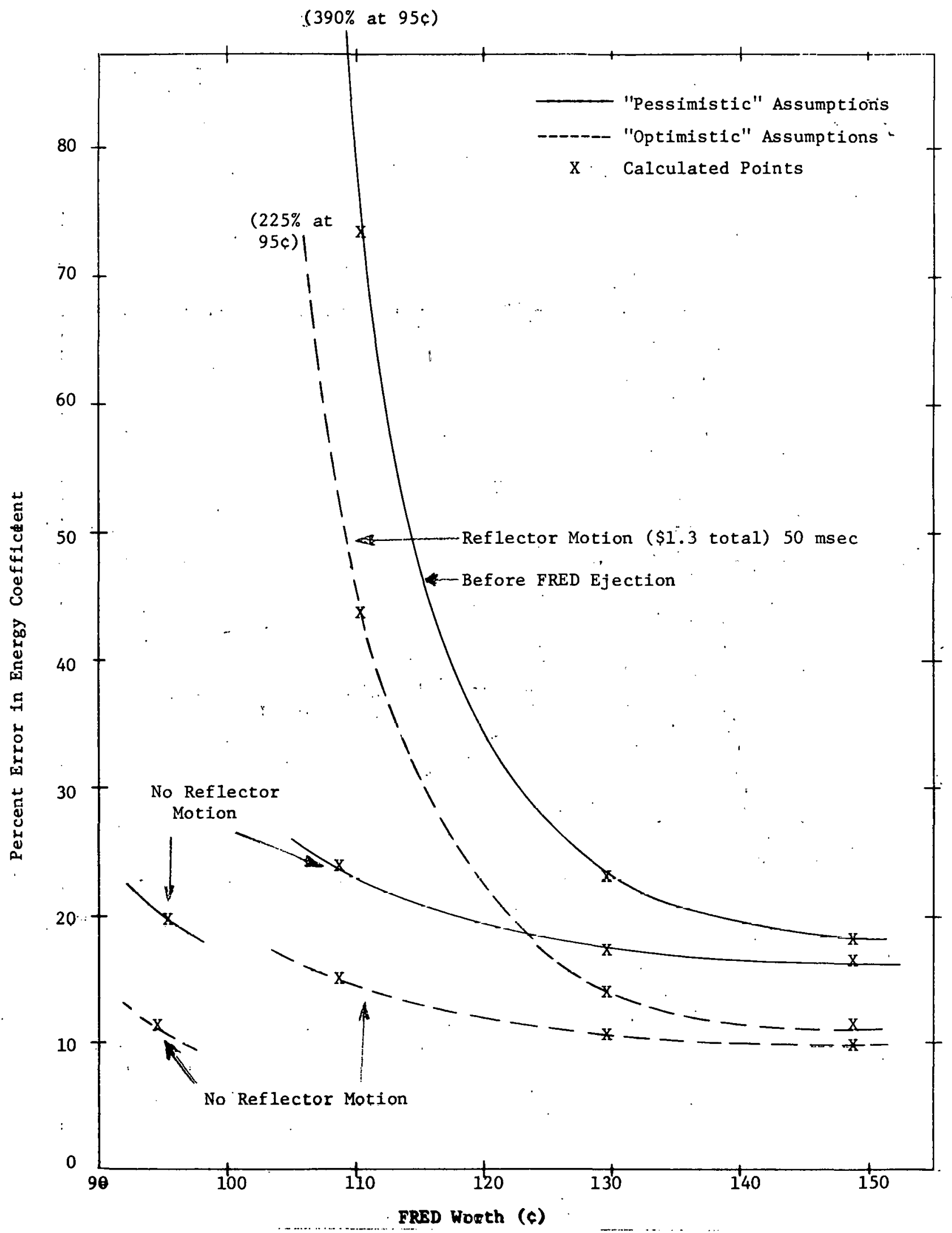

Figure VIII-1. ERROR IN ENERGY COEFFICIENT

VS. FRED WORTH 
GEAP-55.76:

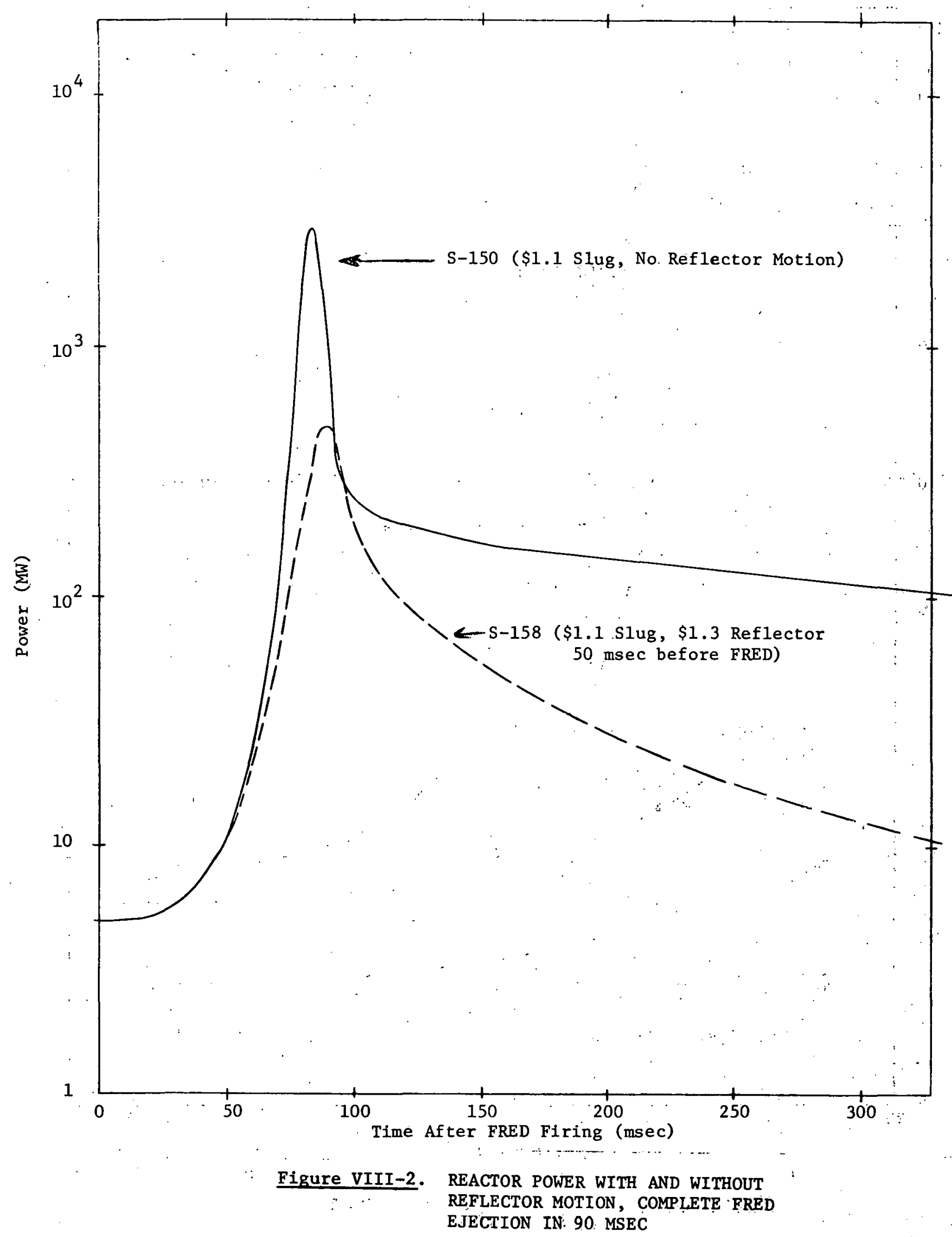

VIII-4 
The fuel axial expansion coefficient corresponds to about twice the value of the axtal expansion effect recently reported in reference (16). The structure and sodium coefficient is approximately one-half of the presently calculated reactivity effect in SEFOR. Any increase in the ratio of the extraneous effects to the Doppler effect would increase (with the same relative uncertainty in the extraneous effects) the uncertainty in the Doppler energy coefficient. A higher initial power would tend to increase the error in the Doppler energy coefficient (lower initial power $\rightarrow$ decrease in error) since the Doppler effect is reduced, relative to other effects, with increasing fuel temperature. Uncertainty in the proper errors to be assumed was accounted for to some extent by the use of the "pessimstic" and "optimistic" assumptions decribed below. In addition to the above values, the time dependent FRED and reflector curves shown in Appendix VII, were used.

For simplicity, it was assumed that the Doppler reactivity effect $\left(\Delta k_{D}\right)$ during a transient can be described by a linear dependence upon the change in energy $(\Delta E)$, i.e.

$$
\Delta \mathrm{k}_{\mathrm{D}}=\gamma \Delta \mathrm{E}
$$

It was also assumed that all errors, Including biases, are uncorrelated and can be propagated as random errors.

The fractional random error in the Doppler energy coefficient $(\gamma)$ is given by:

$$
\frac{\sigma_{\gamma}}{\gamma}=\sqrt{\left(\frac{\sigma_{D}}{\Delta k_{D}}\right)^{2}+\left(\frac{\sigma_{E}}{\Delta E}\right)^{2}}
$$


where $\sigma_{\gamma}$ denotes the error in $\gamma, \sigma_{D}$ the error in $\Delta k_{D}$, etc.

The Doppler reactivity effect $\left(\Delta k_{D}\right)$ can be written as:

$$
\Delta \mathrm{k}_{\mathrm{D}}=\Delta \mathrm{k}_{\mathrm{N}}-\Delta \mathrm{k}_{\mathrm{F}}-\Delta \mathrm{k}_{\mathrm{r}}-\Delta \mathrm{k}_{\mathrm{e}}-\Delta \mathrm{k}_{\mathrm{o}}
$$

where

$$
\begin{aligned}
& \Delta \mathrm{k}_{\mathrm{N}}=\text { the net change in reactivity (calculated in SEFOR } \\
& \text { experiments by inversion of the kinetic equation) } \\
& \Delta \mathrm{k}_{\mathrm{F}}=\text { FRED reactivity } \\
& \Delta \mathrm{k}_{\mathrm{r}}=\text { reflector reactivity } \\
& \Delta k_{e}=\text { fuel axial expansion effect } \\
& \Delta \mathrm{k}_{\mathrm{o}}=\mathrm{clad}+\text { coolant and structure effect (lumped together } \\
& \text { in the error analyses, but not in the FORE calculations). }
\end{aligned}
$$

The rardom error in the Doppler effect is thus given by:

$$
\sigma_{D}=\sqrt{\sigma_{N}^{2}+\sigma_{F}^{2}+\sigma_{\tilde{r}}^{2}+\sigma_{e}^{2}+\sigma_{o}^{2}}
$$

for the super-prompt tests. Rather than looking at the change in. reactivity. from time zero (the time when FRED or reflector is fired), it was assumed for the sub-prompt tests that the data analysis is performed by looking at the difference between the reactivity at a time $t_{0}+\Delta t$ and the reactivity at time $t_{0}$, where $t_{0}$ is the time at which complete FRED ejection occurs. In this case, $\sigma_{F}=0$. The latter procedure can also be applied to the super-prompt tests; however, there is 
some question as to its usefulness, since it yielded smaller errors than those shown in Figure VIII-1, when the "optimistic" assumptions were used, but larger errors when the "pessimistic" assumptions were used.

To first order, the error in the net reactivity during a transient should be proportional to $\left|\Delta \mathrm{k}_{\mathrm{N}}-1\right|$, where $\Delta \mathrm{k}_{\mathrm{N}}$ is in dollars, and the fractional error in FRED and in the reflector worth should be constant. In addition, it was also assumed that the fractional error in the expansion effect, clad + sodium and structure, and change in energy release are constant.

VIII. 2 "PESSIMISTIC" ASSUMPTIONS

For the two-segment SEFOR core, the fractional error in the fuel expansion effect is about 0.35 . Using the same fractional uncertainty, 0.35 , and twice the expansion effect should yield a pessimistic absolute value of the fuel expansion error. For the other reactivity effects (clad + sodium and structure) a fractional error of 1.0 was assumed. This is larger than $a \pm 100 \%$ uncertainty in each, since these reactivity effects were summed before propagating the error. The uncertainty in $\Delta \mathrm{k}_{\mathrm{N}}$ was assumed to be $10 \%$ of $\left|\Delta \mathrm{k}_{\mathrm{N}}-1\right|$, (the expected error is < $5 \%$ ) and a fractional error of 0.05 was assumed for the change in energy. The latter repreșents $\sim 3 \%$ bias error and $\sim 0$ random error.

It was assumed that the FRED and one reflector can be calibrated with less than 5\% error. The FRED calibration was assumed to be slightly better than the reflector calibration, since the reflector strength may be more sensitive to the position of the other reflectors. Should the error in FRED exceed $\sim 5 \%$, the qualitative conclusions given above, and the qualitative behavior of the curves in Figure VIII-1 would remain unchanged. 
The form and magnitude of the various errors are listed below.

$$
\begin{aligned}
& \sigma_{\mathrm{e}} / \Delta \mathrm{k}_{\mathrm{e}}=0.35 \equiv \mu_{\mathrm{e}} \\
& \sigma_{\mathrm{o}} / \Delta \mathrm{k}_{\mathrm{o}}=1.0 \equiv \mu_{\mathrm{o}} \\
& \sigma_{\mathrm{N}}=0.10\left|\Delta \mathrm{k}_{\mathrm{N}}-1\right| \equiv \mu_{\mathrm{N}}\left|\Delta \mathrm{k}_{\mathrm{N}}-1\right| \\
& \sigma_{\mathrm{F}} / \Delta \mathrm{k}_{\mathrm{F}}=0.03 \equiv \mu_{\mathrm{F}} \\
& \sigma_{\mathrm{r}} / \Delta \mathrm{k}_{\mathrm{r}}=0.04 \equiv \mu_{\mathrm{r}} \\
& \sigma_{\mathrm{E}} / \Delta \mathrm{E}=0.05 \equiv \mu_{\mathrm{E}} .
\end{aligned}
$$

These assumptions were used with the appropriate numbers obtained.. from the FORE output to calculate the numbers shown in Figure VIII-I.

\section{VIII.3 : "OPTTMMSTIC" ASSUMPTIONS}

For this case, it was assumed that the magnitude of all the errors listed above was decreased by a factor of 2 , except for the energy release and the FRED worth. The error in the energy was unchanged and the error in the FRED worth which was decreased to $2 \%$. The assumed form and magnitude are listed below.

$$
\begin{array}{ll}
\sigma_{\mathrm{e}} / \Delta \mathrm{k}_{\mathrm{e}}=0.175 & \sigma_{\mathrm{r}} / \Delta \mathrm{k}_{\mathrm{r}}=0.02 \\
\sigma_{\mathrm{o}} / \Delta \mathrm{k}_{\mathrm{o}}=0.50 & \sigma_{\mathrm{E}} / \Delta \mathrm{E}=0.05 \\
\sigma_{\mathrm{N}}=0.05\left|\Delta \mathrm{k}_{\mathrm{N}}-1\right| & \\
\sigma_{\mathrm{F}} / \Delta \mathrm{k}_{\mathrm{F}}=0.02 &
\end{array}
$$




\section{VIII.4 SAMPLE CALCULATIONS}

Listed below are the FORE output at a time $\dot{\sim} 60 \mathrm{msec}$ after FRED has been completely ejected for cases in which a $\$ 1.1$ slug was fired with and without reflector motion.

\section{TABLE VIII-1}

Case S-150 ( $\$ 1.10 \mathrm{slug}$ - no reflector motion - FRED out at $t=90 \mathrm{msec})$

\begin{tabular}{ccccccc}
$t$ & $\Delta k_{\mathrm{D}}$ & $\Delta \mathrm{k}_{\mathrm{e}}$ & $\Delta \mathrm{k}_{\mathrm{o}}$ & $\Delta \mathrm{k}_{\mathrm{F}}$ & $1-\Delta \mathrm{k}_{\mathrm{N}}$ & $\Delta \mathrm{k}_{\mathrm{r}}$ \\
(msec) & $(\mathrm{c})$ & $(\mathrm{c})$ & $(\mathrm{c})$ & $(\mathrm{c})$ & $(\mathrm{c})$ & $(\mathrm{c})$ \\
\hline 154.0 & 18.82 & 4.32 & 1.51 & 110.0 & 14.65 & 0.0
\end{tabular}

TABLE VIII-2

Case S-158 ( $\$ 1.10 \mathrm{slug}$ - reflector motion $50 \mathrm{msec}$ before FRED FRED out at $t=140 \mathrm{msec}$ )

$\begin{array}{cccccccc}t & \Delta \mathrm{k}_{\mathrm{D}} & \Delta \mathrm{k}_{\mathrm{e}} & \Delta \mathrm{k}_{\mathrm{o}} & \ddots & \Delta \mathrm{k}_{\mathrm{F}} & 1-\Delta \mathrm{k}_{\mathrm{N}} & { }^{\Delta \mathrm{k}_{\mathrm{r}}} \\ \text { (msec) } & (c) & (c) & (c) & (c) & (c) & (c) \\ 197.5 & 6.23 & 1.39 & .46 & 110.0 & 20.33 & 22.25\end{array}$


GEAP -5576

TABLE VIII-3

\section{Calculated Errors}

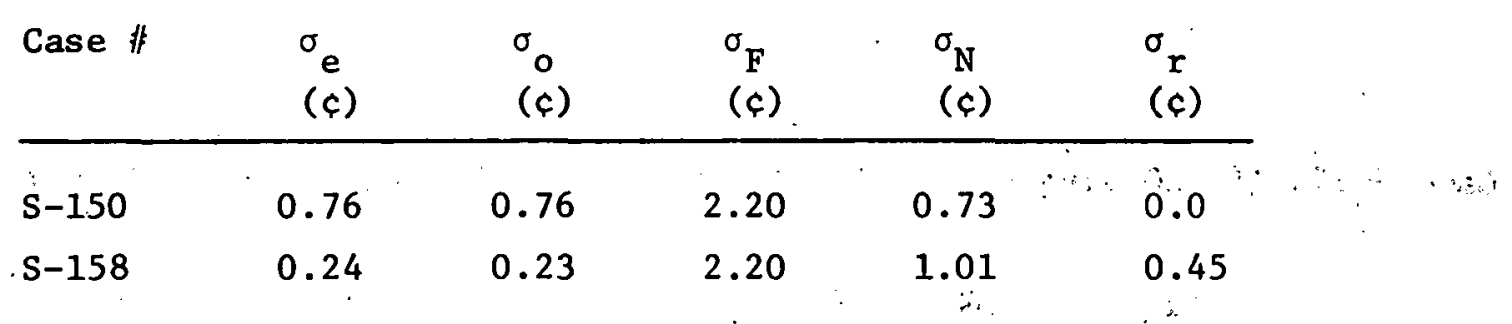

Since $\sigma_{D}$ is the square root of the sum of the squares of the above numbers, one obtains

$$
\begin{aligned}
& \sigma_{D}=\sqrt{6.53}=2.56 c \cdot(\mathrm{S}-150) \\
& \sigma_{D}=\sqrt{6.17}=2.48 \mathrm{c}(\mathrm{S}-158)
\end{aligned}
$$

Since

$$
\frac{\sigma_{\gamma}}{\gamma}=\sqrt{\left(\frac{\sigma_{D}}{\Delta k_{D}}\right)^{2}+\left(\frac{\sigma_{E}}{\Delta E}\right)^{2}}
$$

one obtains:

$$
\begin{aligned}
& \frac{\sigma_{\gamma}}{\gamma}=\sqrt{\left(\frac{2.56}{18.82}\right)^{2}+(.05)^{2}}=0.145(\mathrm{~s}-150) \\
& \frac{\sigma_{\gamma}}{\gamma}=\sqrt{\left(\frac{2.48}{6.23}\right)^{2}+(.05)^{2}}=0.407(\mathrm{~s}-158)
\end{aligned}
$$




\section{VIII.5 ASYMPTOTIC BEHAVIOR OF ERRORS}

The primary reason that the errors in Figure VIII-1 do not decrease significantly for slug sizes $\gtrsim \$ 1.3$ is the following:

During a time interval $\simeq 100 \mathrm{msec}$ after FRED has been completely ejected FORE results for a super-prompt transient show that the rise in clad temperature is $\simeq 10 \%$ of the rise in fuel temperature, and that the rise in sodium and structure temperature is $\simeq 6 \%$ of the fuel temperature rise. In addition, the total feedback reactivity, in cents; can be approximated, very roughly, by $\Delta k_{F}-85$, where $\Delta k_{F}$ is the FRED worth $(~(110)$ in cents. If the Doppler effect is approximated by a linear coefficient $A / T_{0}$, where $A$ is the Doppler $T d k / d T$ and $T_{0}$ is the initial fuel temperature, then all feedback effects are proportional to the fuel temperature rise and can thus be written in terms of the Doppler effect. Using the above assumptions, the square of the fractional error in the Doppler energy coefficient can be approximated by:

$$
\begin{array}{r}
\left(\frac{\sigma_{\gamma}}{\gamma}\right)^{2} \approx\left(\frac{\sigma_{E}}{E}\right)^{2}+\left(\frac{T_{o}}{A}\right)^{2}\left[\left(\mu_{e} \alpha_{e}\right)^{2}+\left(0.1 \mu_{c} \alpha_{c}\right)^{2}+\left(0.06 \mu_{s} \alpha_{s}\right)^{2}\right] \\
+\left[1+\frac{T_{o}}{A}\left(\alpha_{e}+.1 \alpha_{c}+.06 \alpha_{s}\right)\right]^{2}\left[\mu_{N}^{2}\left(\frac{15}{\Delta k_{F}-85}\right)^{2}+\mu_{F}^{2}\left(\frac{\Delta k_{F}}{\Delta k_{F}-85}\right)^{2}\right]
\end{array}
$$


where the $\mu^{\prime}$ s are the fractional uncertainties in the various effects." (see "Pessimistic" Assumptions), and the $\alpha$ 's are the various reactivity coefficients (see Reactivity Effects). For the coefficients and assumptions used here (lumping of clad, coolant and structure errors) the above expression reduces to the following for the "pessimistic" values of the $\mu$ 's.

$$
\frac{\sigma_{\gamma}}{\gamma} \approx \sqrt{.01823+1.78\left[.01\left(\frac{15}{\Delta \mathrm{k}_{\mathrm{F}}-85}\right)^{2}+\mu_{\mathrm{F}}^{2}\left(\frac{\Delta \mathrm{k}_{\mathrm{F}}}{\Delta \mathrm{k}_{\mathrm{F}}-85}\right)^{2}\right]}
$$

This can be used to generate the following table.

$\begin{array}{lcccccc}\Delta \mathrm{k}_{\mathrm{F}}(c) & 110 & 130 & 150 \cdots & 170 & \ldots & \infty \\ \sigma_{\gamma} / \gamma\left(\mu_{F}=.03\right) & .236 & .183 & .167 & .159 & .141 \\ \sigma_{\gamma} / \gamma\left(\mu_{F}=.06\right) & .385 & .272 & .231 & \ldots .211 & \vdots & .157\end{array}$

Although the above numbers do not agree exactly with the curves in ' Figure VIII-1, they do indicate the trend that occurs with increasing slug size. 


\section{IX.1 INTRODUCTION}

Because of the $\mathrm{Be}^{9}(\gamma, \mathrm{n}) \mathrm{Be}^{8}$ reaction, photoneutrons will be produced in SEFOR. Those photoneutrons, which appear as a result of radioactive decay of fission products, or as a result of beta decay of activated core materials, will appear in the kinetics equations as extra groups of delayed neutrons. The effect of these extra groups on the kinetic behavior of the SEFOR reactor is described below.

IX.2 RESULTS AND CONCLUSIONS

In SEFOR (mass fraction of $B e$ in core $=0.0089$ ) the delayed photoneutron fraction $\left(\beta_{p}\right)$ is $\leqslant 2 \times 10^{-6}$, wh1le the total effective delayed neutron fraction $(\bar{\beta})$ i.s. $3.2 \times 10^{-3}$. Thus, the contribution of the photoneutrons to $\vec{\beta}$ is approximately $(100)\left(2 \times 10^{-6}\right) /\left(3.2 \times 10^{-3}\right)$ $\approx 0.06 \%$. Although some of the delayed photoneutron groups have 1ong half lives, the difference between the zero, power transfer function at $0.00125 \mathrm{cps}$, with and without photoneutrons, is on $1 \mathrm{y}$ about $0.2 \%$ in amplitude and 0.2 degrees in phase. The difference is even less at higher frequencies, and in any event is probably less than the uncertainties in the experiments.

Since the delayed photoneutron effects are small in SEFOR, they can be neglected except for the following exception: Because of their long half lives, continued operation at high power will generate a source 
of neutrons which, if the reactor is subsequently operated at low power, will slowly decay away. Since the effective source strength effects the criticality of the system, experiments at low power should not be immediately preceded by continued operation at high power (e.g., Discussion).

IX. 3 DISCUSSION

By fitting the photoneutron data of Bernstein, et al, (17) the value of the delayed photoneutron fraction $\left(\beta_{p}\right)$ obtained by Keepin. ${ }^{(18)}$ is $1.5175 \times 10^{-4}$ for a system in which all the gamma rays that produce photoneutrons are absorbed in Be. If it is assumed that the mass attenuation coefficient is constant for all materials, then the delayed photoneutron fraction for SEFOR (mass fraction of $B e=0.0089$ ) is $\left(1.5175 \times 10^{-4}\right)(0.0089)=1.35 \times 10^{-6}$. The preceding calculation neglects gamma leakage from the core and gamma rays from activated core materials. Both effects are small.

In experiments performed in reactors containing mass fractions of Be between $\sim 0.6$ and 0.9 Krashin, et al, ${ }^{(19)}$ obtained a value for the delayed photoneutron fraction of $1.48 \times 10^{-4}$. Krashin does not state whether this value of $\beta_{p}$ is normalized to an infinite amount of $B e$ or whether it was the value obtained for some specific reactor configuration: Assuming (conservatively) that Krashin's value is for the reactor with the smallest mass fraction of $B e$, one obtains $\beta_{p}(S E F O R)=$ $\left(1.48 \times 10^{-4}\right)(0.0089) / 0.6=2.2 \times 10^{-6}$.

Since the effective delayed neutron fraction $(\bar{\beta})$ for SEFOR is $3.2 \times 10^{-3}$, the contribution of $\beta_{p}$ to $\bar{B}$ is only $0.06 \%$. In order to determine the effect of the SEFOR delayed photoneutrons, the zero. 
power transfer function was computed at several frequencies between 0.00125 and 0.5 cps. Keepin's ${ }^{(18)}$ delayed neutron parameters for Pu-239, $\mathrm{U}-238$ and $B e$ photoneutrons (normalized to value of $B_{\mathrm{p}} / \bar{B}=6.1 \times 10^{-4}$ ) were used. The maximum difference in the transfer function with, and without delayed photoneutrons was $\approx 0.2 \%$ in amplitude and 0.2 degrees in phase at $0.00125 \mathrm{cps}$.

In order to give an explicit example of the effect of the delayed photoneutrons, assume for simplicity that the reactor was operated at very low power for a long period of time, then operated at a high power $P_{1}$ for time $T$, and shut down to power $P_{0}$ at time $t=0$. The reactivity (in dollars) required to hold the power constant at $P_{0}$ is (in the absence of a source) then given by

$$
k(t)=-\sum_{i} \frac{\beta_{i}}{\bar{\beta}} e^{-\lambda_{i} t}\left[\frac{P_{1}}{P_{0}}\left(1-e^{-\lambda_{i} T}\right)-1\right]
$$

where $t$ is the time that has elapsed since the reactor was instantaneously shut down to power $P_{0}$, and the index $i$ runs over all precursor groups (including photoneutrons).

After the first 10 minutes or so all the fission product delayed neutrons attain their new equilibrium values corresponding to the power P. However, the delayed photoneutrons, because of their long half lives do not achieve equilibrium within 10 minutes and $k(t)$ will be negative (i.e. the reactor will be subcritical) for quite some time. 
Using the same delayed neutron parameters that were used to calculate the SEFOR transfer function (see above) the folloring table can be constructed for the case $\mathrm{P}_{1}=20 \mathrm{MW}, \mathrm{P}_{0}=100 \mathrm{kw}$. If $P_{0}=10 \mathrm{kw}$, the reactivity values listed below must be multiplied by a factor of 10 .

\begin{tabular}{|c|c|c|}
\hline & & \\
\hline & $\mathrm{T}=10 \mathrm{hrs}$. & $T=\infty$ \\
\hline $10 \mathrm{~min}$ & -4.9 & $\begin{array}{r}: \\
-5.4 \\
\\
\quad-4\end{array}$ \\
\hline $30 \mathrm{~min}$ & -3.1 & -3.5 \\
\hline $1 \mathrm{hr}$ & -2.2 & -2.6 \\
\hline $2 \mathrm{hr}$ & -1.6 & -2.0 \\
\hline $3 \mathrm{hr}$ & -1.3 & -1.6 \\
\hline $4 \mathrm{hr}$ & -1.0 & -1.3 \\
\hline $8 \mathrm{hr}$ & -0.44 & -0.64 \\
\hline $24 \mathrm{hr}$ & -0.036 & -0.13 \\
\hline
\end{tabular}

As the table indicates, the reactor will be subcritical for an extended length of time. In order to avoid the complication of having to know the exact delayed photoneutron parameters, a waiting period of from 8 to 24 hours after operation at high power should clapse before any oscillator experiments or rod calibrations are performed in the $10 \mathrm{kw}$ to $100 \mathrm{kw}$ power range. 
GEAP-5576

\section{REFERENCES}

Sections I and II

1. G. R. Pflasterer, "SEFOR Task 1.1 Topical Report; Specifications for the Plant and Experiment Equipment," GEAP-5092, Vo1. I.

2. G. R. Pflasterer, and L. Caldarola, "SEFOR Task 1.1 Topical Report, Test Description," GEAP-5092, Vol. II.

3. W. Hafele, K. Ott, L. Caldarola, W. Schikarski, K.P. Cohen, B. Wolfe, P. Greebler, and A.B. Reynolds, "Static and Dynaintc Measurements of the Doppler Effect in an Experimental Fast Reactor," Geneva Conference, 1966, A/Conf./28/p/644.

4. B. Wolfe, K. Hikido, K. Horst, and A.B. Reynölds, "SEFOR - A Status Report," ANL-100, Fast Reactor Technology Nat1onal Topical Meeting, Detroit (1965).

5. L. D. Noble, G.R. Pflasterer, C.D. Wilkinson, and L. Caldarola, "Recent Developments in the SEFOR Experimental Program," ANL-7320, Proceedings of the InLerhational Cunference on Fast Critical Experiments and Their Analysis, Argonne, Illino1s, October, 1966.

6. L. Caldarola, "The Balanced Oscillator Experiment," KFK-253, Kar1sruhe, Germany; "First Balanced Oscillator Experiment," IAR Notiz No. 10, Karlsruhe, Germany; "Second Balanced Oscillator Experiment," IAR Notiž No. 17, Karlsruhe, Germany. 
GEAP -5576

\section{References (Cont.)}

7. GEAP-5301, "Southwest Experimental Fast Oxide Reactor Development Program, Tenth Quarterly Report, August-October, 1966," Section 3.2.3.9.

8. GEAP-5442, "Southwest Experimental Fast 0xide Reactor Development Program, Eleventh Quarterly Report, November 1966-January 1967," Section 10.3.

9. F. Storrer, "Fission Product Decay Heat and Reactor Kinetics," Trans. of Am. Nuc. Soc., Vol. 3.2 (1960).

10. D.F. Schoeberle, J. Heestand, and L.B. Millex, "A Method of Calculating Transient Temperatures in a Multiregton, Axlsymetrlc, Cylindrical Configuration. The ARGUS Program, 1089/RE284," ANL-6654 (1963).

11. P. Greebler, D.B. Sherer, and N.H. Walton, "FORE, A Computational Program for the Analysis of Fast Reactor Excursions," GEAP-4090.

12. D. Berman and C.D. Wilkinson, "Weighted Doppler Analysis Code WEDOP," GEAP-5513. 
GEAP -5576

References (Cont.)

\section{Section III}

1. M. Edelmann, et a1, "Pulsed Source and Noise Measurements on the STARK Reactor at Karlsruhe," KFK-303, May, 1965.

2. R.P. Morre11, "SEFOR $B / \ell$ Measurements by Noise Analysis Experiments," IDO-17210.

3. G.R. Keepin, "Physics of Nuclear Kinetics," Addison-Wesley, Palo Alto (1965).

4. GEAP-5442, "Southwest Experimental Fast Oxide Reactor Development Program, Eleventh Quarterly Report, November 1966-January 1967."

5. A.B. Reynolds and S.L. Stewart, "Analysis of the SEFOR Mockup Critical Experiments in ZPR-III," GEAP-5294.

6. R.L. McVean, et a1, "Critical Studies of the Southwest Experimental Fast Oxide Reactor (SEFOR) in ZPR-3 (Assemb1y 47)," ANL-7248, March, 1967.

7. J.M. Cooley and J.W. Tukey, "An Algorithm for the Machine Calculation of Complex Fourier Series," Mat. of Comput., Vo1. 19, pp 297-301, Apri1, 1965.

8. J.B. Dragt, "Analysis of Reactor No1se Measured in a Zero-Power Reactor and Calculations on Its Accuracy,". CONF-660206, Proceedings of a symposium at the University of Flordia, Gainesville, February 14-16, 1966. 


\section{References (Cont.)}

\section{Section IV}

1. op cit - Reference 12 , Section

2. op cit - Reference 7, Séction I and II.

\section{Section V}

1. op cit - Reference 6 , Section I and II.

2. GEAP-5301; "Southwest Experimental Fast Oxide Reactor Development Program, Tenth Quarteriy Report, August-october, 1966," Section 3.2 .3 .4 .

3. op cit - Reference 1, Section I and II.

4: R. Persson, "Method of Eliminating the Aperiodic Pattern" of a Fourier Analysis, for Example in Meásurements with a Pile-oscil1ator," ANL-Trans-43, (English translation of Swedish document AEFI-54).

5. M.A. Schultz, "Control of Nuclear Reactors and" Power Plants," McGraw-Hi11, New York (1961).

6. W.T. Thompson, "Laplace Transformation," Prentice-Ha11," Englewood Cliffs, . New Jersey' (1960). 
GEAP-5576

References (Cont.)

7. H.A. Bethe, "Reactor Safety and Oscillator Tests," APDA-117 (1956).

8. V.V. Solodovnikov, "Introduction to the Statistically Dynamics of Auţomatic Contrọl Systems," Dover, New., York (1960). .

9. G.R. Keepin, "Physics of Nuclear Kinetics," Addison-Wesley, Palo Alto (1965).

10. op cit - Reference 8, Section I and II.

11. GEAP-5208, "Southwest Experimental Fast Oxide Reactor Development Program, Ninth Quarterly Report, May-July 1966," Section. 3.2.4.

12. W.E. Deming, "Statistical.Adjustment of Data," Dover, New York (1943).

13. op cit - Reference 7, Section I and II, Section 3.2.3.4. 


\section{References (Cont.)}

\section{Section VI}

1. J.W. Riese and G. Collier, "VARI-QUIR: A Two-Dimensional TimeDependent Multi-Group Diffusion Code," WANL-TNR-133 (1963).

2. op cit - Reference 7, Section I and II, Section 3.2.3.3.

3. Power, B.J., et a1, "SPORT - A System for Processing Reactor Transient Data on the IBM-7040 Computer," IDO-17078 (1965).

4. op cit - Reference 10, Section I and II.

5. op cit - Reference 11 , Section I and II.

\section{Appendiëes}

1. op cit - Reference 9, Section I and II.

2. Op cit - Reference 8, Section I and .II.

3. S. Glasstone and A. Sesonske, "Nuclear Reactor Engineering," D. va Nostrand, Princeton (1965).

4. J.N. Fox, B.E. Lawler, and H.R. Butz, "FORE-II - A Computational Program for the Analysis of Steady-State and Transient Reactor Performance," GEAP-5273 (1966). 
GEAP -5576

References. (Cont.)

5. op cit - Reference 6, Section I. and II.

6. C. Russel1 - Appendix A, "'Facility Description and Safety: Analysis Report for SEFOR," Vol. II.

7.: op cit - Reference 4, Section V.

8. R.B. Blackman, "Linear Data-Smoothing and.Prediction: in Theory: and Practice," Addison-Wesley, Palo Alto (1965).

9. R.B., Blackman: and J.W. Tukey, "The Measürement ,of:.Power. Spectra from the Point of View of Communications Engineering," Dover, New York (1958).

10. op cit - Reference 12 , Section $V$.

11. F.B. Hildebrand, "Introduction to Numerical Analysis," McGraw-Hill, New York (1956), Chapter 7.

12. W.A. Zschaler - private communication.

13. GEAP-5160, "Southwest Experimental Fast Oxide Reactor Development Program, Eighth Quarterly Report, February-Apri1, 1966."

14. E.R. McKeehan - private communication - see also Reference 2, Section V, Section 8.3. 


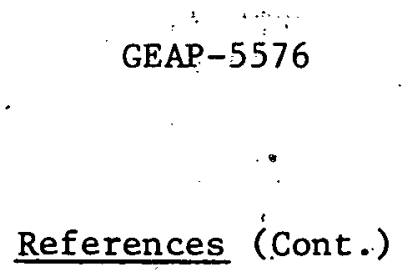

15. op cit - Reference 11, Section I and II.

16. op cit - Reference 8 , Section I and II.

17. S. Berstein, et a1, "Yield of Photoneutrons from U-235 Fission Products in Beryllium and Deuterium," J. App1: Phys. 27,:18 (1956).

18. op cit - Reference 9 , Section V.

19. A.K. .Krashin, et al, "Physical Characteristics of Beryllium Moderated Reactor," Proc. 2nd Geneva Conference 12; 571: (1958). 


\section{ACKNOWLEDGEMENTS}

The work reported here is based on the contributions of-many. people.

Personnel of the Karlsruhe Laboratory have contributed significantly to the analysis and planning of the experiments. The chief contributors from the Karlsruhe Laboratory are:
L. Caldarola
P. Giordano
W. Glauner
W. Hafele

The chief contributors from the General Electric Company are:
J. Arterburn
E. McKeehan
K. Cohen
R. Meyer
E. Craig
G. Pflasterer
P. Greebler
A. Reynolds
K. Hikido
C. Russe11
K. Horst
S. Stewart
B. Wolfe 
Division of 'lechnical Information Extension

U. S. Atomic Energy Commission

P. O. Box 62

Oak Ridge, Tennessee

Director

Research and Development Division

U. S. Atomic Energy Commission

Richland Operations office

P. O. Box 550

Richland, Washington 99352

Mr. G. Vendryes

Cen Saclay

Boite Postale. 2

Gif-Sur-Yvett ( $S$ et 0 ) France.

Dott, Ing. F. Perantoni

CNEN

Via Mazzini 2

Bologna, Italy

Mr. E. E. Kintner, Chief

Fuel Fabrication Branch

DRD\&T, USAEC

Washington, D. C. 20545

Mr. W. O. Turner,

Chairman of Board

Louisiana Power and Light Company

142 Delaronde Street

New Orleans, Louisiana

Mr. R. C. Green, President

10700 East 50 Highway

Kansas City, Missouri

Mr. L. J. Cucullu, Vice President

New Orleans Public Service Inc.

317 Baronne Street

New Orleans, Louisiana

Mr. B. S. Jeffrey, President

Kansas Power and Light Company

$808^{\circ}$ Kansas Avenue

Topeka, Kansas 


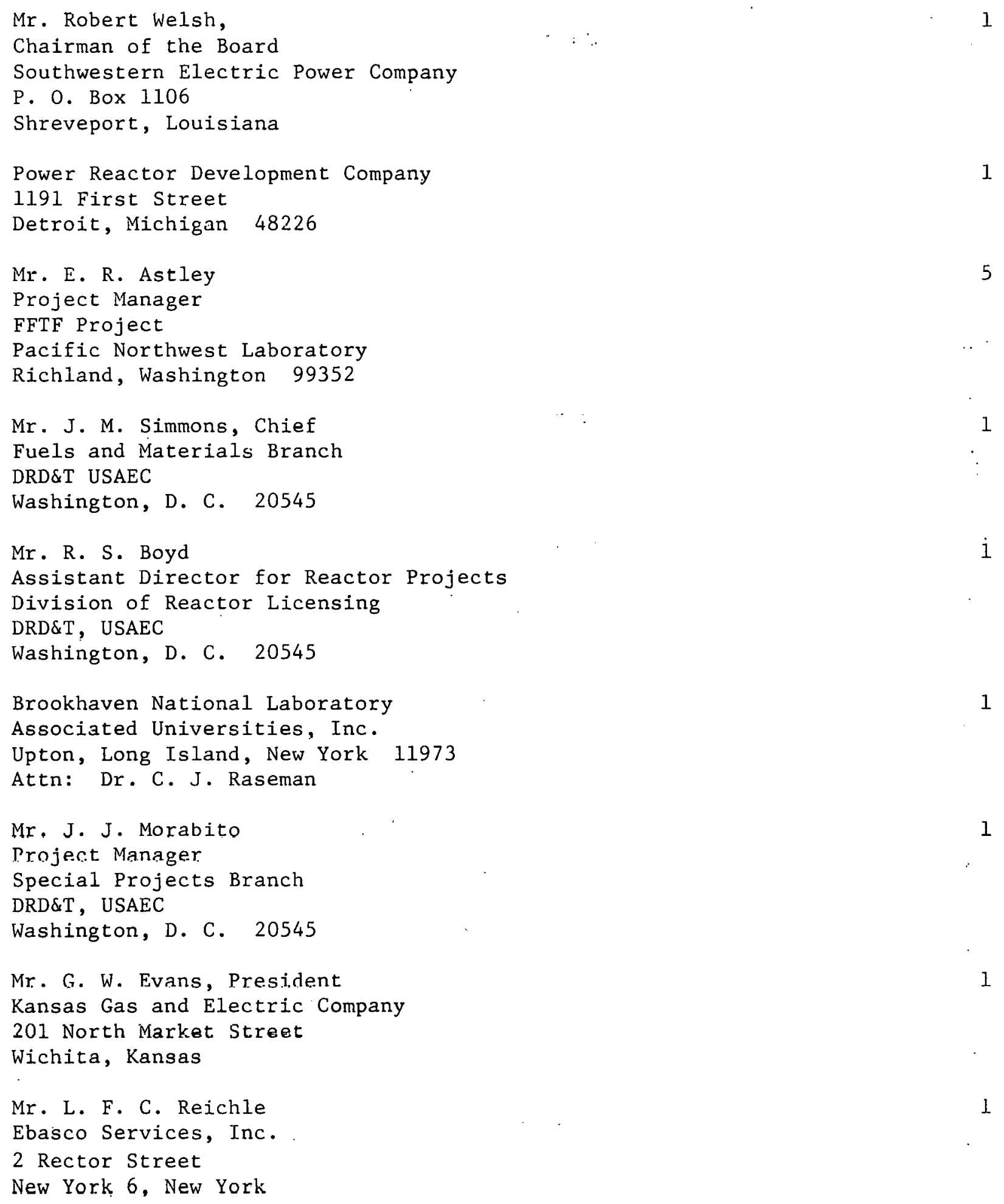


Mr. R. B. Wilson, President

Mississippi Power and Light Company

Electric Building

Jackson, Mississippi

Mr. Ray W. Call, President

Missouri Utilities Company

400 Broadway

Cape Girardeau, Missouri

Mr. D. S. Kennedy, President

Oklahoma Gas and Electric Company

321 North Harvey Street

Oklahoma City, Oklahoma

Mr. D. J. Tuepker, President

Public Service Company of Oklahotina

600 South Main Street

Tulsa, Oklahoma

Dr. G. W. Wensch

Chief, Liquid Metal Project Branch

DRD\&T

U. S. Atomic Energy Commission

Washington, G. C. 20545

Dr. J. A. Lieberman

Asst. Director for Nuclear Safety

DRD\&T

Washington, D. C. 20545

Director, Contracts Division

U. S. Atomic Energy Commission

San Francisco Operations office

2111 Bancroft Way

Berkeley, California 94704

F. W. Thalgott

Associate Director,

Idaho Division

Argonne National Laboratory

Box 1096

Idaho Falls, Idaho 83401

Director, Argonne National Laboratory

9800 South Cass Avenue

Argonne, Illinois 60439

Attin: S. Lawrowski 


\section{DISTRIBUTION LIST (Continued).}

Director

Los Alamos Scientific Laboratory

Los Alamos, New Mexico 87544

Dr. John C. Woodhouse

I Guest Lane

Wilmington, Delaware 19809

Senior Representative

U. S. Mission to EURA'IOM Communities

c/o U. S. Embassy

Brussels, Belgium

General Directorate

Research and Training

EURATOM

51-53 Rue Belliard

Brussels, Belgium

Attn: A. DeStordeur

Mr. F. H. Coughlin, President

Central Louisiana Electric Co., Inc. 415 Main Street

Pineville, Louisana

Mr. John Stewart, President

The Central Kansas Power Company

111 East 11th Street

Hays, Kansas

Mr. Reeves Ritchie, President

Arkansas Pnwer and Light Company

Ninth and Loulsiana Street

Little Rock, Arkansas

Mr. J. T. Iones, President

The Empire District Electric Co.

602 Joplin Street

Joplin, Missouri

Mr, Harold F. Mnrtimer

Assistant to Chairman of the Board

Gulf States Utilities Company

P. O. Box 2951

Beaumont, Texas

Mr. C. F. Fdwards, Vice President

Western Power and Gas Company, Inc.

P. O. Box 170

Great Bend, Kansas 
Gesellschaft für Kernforschung in.b.h.

Projekt Schneller Bruter

75) Karlsruhe, Germany

$$
\text { Postfach } 947
$$

Mr. A. Amorosi, Director

LMFBR Program Office

Argonne National Laboratory

9800 South Cass Avenue

Argonne, Illinois 60439

Mr. J. Crawford

Asst. Director for Plant Engineering

DRD\&T, USAEC

Washington, D. C. 20545

Dr. I. Zartman, Chief

Reactor Physics Branch

DRD\&T, USAEC

Washington, D. C. 20545

Mr. C. C. Czeschin, President

Arkansas-Missouri Power Company

104 South Fifth Street

Blytheville, Arkansas

Mr. T. Flynn

Ebasco Services, Inc.

2 Rector Street

New York City 6, New York

USAEC

Office of RDT Site Representative

310 De Guigne Drive

Sunnyvale, California 94086

Attn: C. R. Eisenschmidt, Jr.

Director

Liquid Metals Information Center

P. O. Box 309

Canoga Park, California 91305

Mr. S. Golan, Project Manager

1000 MWe LMFBR Follow-On Study

Atomics International

P. 0. Box 309 .

Canoga Park, California 91304 
GEAP-5576

Mr. M. W. Croft, Project Manager

1000 MWe LMFBR Follow-On Stuḍy

The Babcock and Wilcox Company

5061 Fort Avenue

P. O. Box 1260

Lynchburg, Virginia 24505

Dr. W. P. Staker, Project Mañager

1000 MWe LMFBR Follow-On Study

Combustion Engineering, Inc:

P. O. Box 500

Windsor, Connecticut 06095

Mr. C. A. Anderson, Project Manager

1000 MWe LMFBR Follow-On Study

Westinghouse Electric Corporation

Advanced Reactors. Division

Waltz Mill Site

P. 0. Box 158

Madison, Pennsylvania 15663

Mr. L. W. Fromm, Manager

1000 MWe LMFBR Follow-On Study Project

Building 208

Argonne National Laboratory

9800 South Cass Avenue

Argonne, Illinois 60439

USAEC Scientific Representative

United States Embassy

London, England

Mr. M. A. Rosen

Asst. Director for Program Analysis

DRD\&T, USAEC

Washington; D. C. 20545 